\title{
Design and Adaptive Control of a Tendon-Driven Manipulator for the Capture of Non-Cooperative Space Targets
}

\author{
by \\ Justin E. Kernot
}

A thesis submitted to the Faculty of Graduate and Postdoctoral Affairs in partial fulfillment of the requirements for the degree of

\author{
Master of Applied Science \\ in \\ Aerospace Engineering
}

\author{
Carleton University \\ Ottawa, Ontario \\ (C) 2019 \\ Justin E. Kernot
}


This work is dedicated to my late grandfather, John Bosworth. 


\begin{abstract}
Orbital debris in Earth orbit poses a threat to the future of spaceflight. To combat this issue, this thesis proposes a novel robotic mechanism for non-cooperative capture and active servicing missions on non-cooperative targets. Specifically, a tendon-driven manipulator designed to employ a synthetic gecko adhesive on the contact interface is proposed. Due to the scale of this project, this thesis explores the design and control of a prototype for use on the Spacecraft Proximity Operations Test-bed at Carleton University's Spacecraft Robotics and Control Laboratory.

This capture mechanism is a symmetric two-link gripper driven by an open-ended cable-sheath transmission mechanism. Using optimal and classical techniques, the gripper was designed and built for the purpose of capturing a free-floating platform in a planar environment. Since the cable-sheath transmission mechanism is a nonlinear time varying hysteretic system, two separate adaptive control strategies were compared against the uncontrolled and PID controlled performance of the closed-loop gripper. Specifically, an indirect control method (denoted AB controller in this work) and a direct $\mathcal{L}_{1}$ controller were employed. Both the simulations and experiments demonstrate that the adaptive controllers show better tracking performance of a joint trajectory over the PID and uncontrolled cases. The experimental results show that the $\mathcal{L}_{1}$ controller performs best under dynamic conditions, while the $\mathrm{AB}$ controller performs best in steady state.
\end{abstract}




\section{Acknowledgements}

My deepest gratitude goes to my supervisor, Steve Ulrich, whose insight and guidance were essential to completing this work. He allowed me to explore my passion in spacecraft robotics by pursuing research that was new for the both of us. I am honoured to have had this experience!

I would also like to thank James Gemlo from Asahi Intecc Co., LTD for generously providing the cables and sheaths for this project. The products exceeded my expectations in terms of their performance, and they were instrumental to the completion of this work.

Next, to my friends and family, thank you for all of your support throughout this project. There were many road-blocks along the way which dampened my spirits, but you were always there to help me stay motivated. I sincerely mean it when I say I couldn't have done this without you.

Lastly, I give the utmost appreciation to my colleague, and friend; Alexander Crain. Without his help, I would likely be in the same place as I was several months ago. As promised, here is a poem to thank you for all your help:

From software to servos controlling the SPOT,

I ran into problems; it happened a lot.

I'd ask for your help with the issues I had, and you'd always reply, "Justin, you should be glad."

"Why?", I would ask, like I didn't already know.

Every time, you would say, "It's just a typo". 


\section{Table of Contents}

Abstract $\quad$ iii

Acknowledgements $\quad$ iv

List of Tables $\quad$ ix

List of Figures $\quad \mathrm{x}$

List of Symbols $\quad$ xxii

List of Acronyms xxviii

Chapter 1 Introduction 1

1.1 Motivation ..................... 1

1.2 Problem Statement . . . . . . . . . . . . . . . . . . . 1

1.3 Previous Work ........................ 2

1.3.1 On-Orbit Capture Mechanisms . . . . . . . . . . . 2

1.3.2 Tendon Driven Manipulators . . . . . . . . . . . . . . . . . . 4

1.4 Thesis Objectives . . . . . . . . . . . . . . . . . . 6

1.5 Contributions . . . . . . . . . . . . . . . . . 7

1.6 Organization ....................... 7

$\begin{array}{lll}\text { Chapter } 2 & \text { End Effector Design } & 9\end{array}$

2.1 High Level Concept . . . . . . . . . . . . . . . . . . . . . 9

2.2 Joint Design . . . . . . . . . . . . . . . . . . . . . 11

2.2.1 Joint Configuration Selection . . . . . . . . . . . . . 11

2.2.2 Optimized Joint Configuration . . . . . . . . . . . . . . . 12

2.3 Actuation Design . . . . . . . . . . . . . . 26

2.3.1 Grip Requirements . . . . . . . . . . . . . . . 27

2.3.2 CSTM Design . . . . . . . . . . . . . . . . 33 
2.3.3 Actuator and Spring Design . . . . . . . . . . . . . . . 37

$\begin{array}{lll}\text { Chapter } 3 & \text { Experimental Facilities } & 39\end{array}$

3.1 SPOT Overview . . . . . . . . . . . . . . . . . . 39

3.2 Gripper Assembly and Integration . . . . . . . . . . . . . . . . 40

3.2.1 Gripper Assembly . . . . . . . . . . . . . . . . . . 41

3.2.2 Actuation and Actuator Bank . . . . . . . . . . . . . . . . . . 42

3.3 Instrumentation and Measurements . . . . . . . . . . . . 45

3.4 Experiment Software . . . . . . . . . . . . . . . . . 49

Chapter 4 Adaptive Control Strategies for CSTMs 53

4.1 System Description and Control Strategies . . . . . . . . . . . . . . 53

4.2 Indirect Adaptive Control from Modified Bouc-Wen Hysteresis Model 54

4.2.1 CSTM Kinematic Model . . . . . . . . . . . . . . . . 55

4.2.2 AB Control Algorithm . . . . . . . . . . . . . . 56

$4.3 \mathcal{L}_{1}$ Control with Hysteresis Compensation . . . . . . . . . . . . . . . 59

4.3.1 System Description . . . . . . . . . . . . . . . . 60

4.3.2 CMAC Architecture . . . . . . . . . . . . . . . 62

4.3.3 $\mathcal{L}_{1}$ Control Architecture . . . . . . . . . . . . . . 64

$\begin{array}{lll}\text { Chapter } 5 & \text { Controller Simulations and Tuning } & 67\end{array}$

5.1 Simulating the Plant . . . . . . . . . . . . . . 67

5.1.1 Setting Up the Simulated Environment . . . . . . . . . . . . 68

5.1 .2 Actuator Model Identification . . . . . . . . . . . . . . . 69

5.1.3 CSTM Modelling and Parameter Estimation . . . . . . . . 73

5.1.4 Full Plant Simulation . . . . . . . . . . . . . . . . 75

5.2 Implementation of PID . . . . . . . . . . . . . . . 77

5.3 Implementation of AB Control . . . . . . . . . . . . . . . 79

5.3.1 Sensitivity Analysis on AB Controller Parameters . . . . . . . 80

5.3.2 Simulation Performance of AB Controller . . . . . . . . . . 85

5.4 Implementation of $\mathcal{L}_{1}$ Control . . . . . . . . . . . . . . . . . . 86 
5.4.1 Sensitivity Analysis of $\mathcal{L}_{1}$ Parameters . . . . . . . . . 87

5.4.2 Simulation Performance of $\mathcal{L}_{1}$ Controller . . . . . . . . . . 93

$\begin{array}{lll}\text { Chapter } 6 & \text { Experimental Results and Discussion } & 94\end{array}$

6.1 Overview of Experiments . . . . . . . . . . . . . . . . . . . 94

6.2 No Control . . . . . . . . . . . . . . . . . . . . . . . . 95

6.3 PID Control . . . . . . . . . . . . . . . . . . . . . . . 100

6.4 AB Control . . . . . . . . . . . . . . . . . . . . 103

$6.5 \mathcal{L}_{1}$ Control . . . . . . . . . . . . . . . . . . . . . 106

$\begin{array}{lll}\text { Chapter } 7 & \text { Conclusion } & 109\end{array}$

7.1 Thesis Summary . . . . . . . . . . . . . . . . . . . . . . . . . . . . 109

7.2 Summary of Contributions . . . . . . . . . . . . . . 110

7.3 A Note on Practicality . . . . . . . . . . . . . . . . . . . 111

7.4 Future Work . . . . . . . . . . . . . . . . . . . . . . . . . . 112

$\begin{array}{ll}\text { Bibliography } & 114\end{array}$

$\begin{array}{ll}\text { Appendix A Working Drawings } & 119\end{array}$

A.1 Gripper Assembly and Parts . . . . . . . . . . . . . . . . . . . 119

A.2 Actuator Bank Assembly and Parts . . . . . . . . . . . . . . . . . . 131

$\begin{array}{lll}\text { Appendix B } & \text { Code } & 138\end{array}$

B.1 Optimization Scripts . . . . . . . . . . . . . . . . . . . . . 139

B.2 Actuator Sizing . . . . . . . . . . . . . . . . . . . 143

B.3 Multi-step Function for Dynamixel Protocol 2.0 . . . . . . . . 145

B.4 Manoeuvre Initialization . . . . . . . . . . . . . . . . . . 150

B.5 Controller Initialization . . . . . . . . . . . . . . . . . . . . . . . 152

$\begin{array}{lll}\text { Appendix C Supplementary Data } & 157\end{array}$

C.1 No Control . . . . . . . . . . . . . . . . . . . . . . . 157

C.1.1 LP Joint Data . . . . . . . . . . . . . . . . . . . . . 157 
C.1.2 LD Joint Data . . . . . . . . . . . . . . . . . . . . 159

C.2 PID Control . . . . . . . . . . . . . . . . . . . . . . 163

C.2.1 LP Joint Data . . . . . . . . . . . . . . . . . . . . . 163

C.2.2 LD Joint Data . . . . . . . . . . . . . . . . . . . . 165

C.3 AB Control . . . . . . . . . . . . . . . . . . . . . 168

C.3.1 LP Joint Data . . . . . . . . . . . . . . . . . . . . 168

C.3.2 LD Joint Data . . . . . . . . . . . . . . . . . . . . . 170

C.4 $\mathcal{L}_{1}$ Control . . . . . . . . . . . . . . . . . . . . . . . 174

C.4.1 LP Joint Data . . . . . . . . . . . . . . . . . . . . . . 174

C.4.2 LD Joint Data . . . . . . . . . . . . . . . . . 176 


\section{List of Tables}

2.1 Optimal Solutions for Each Case. . . . . . . . . . . 23

2.2 Performance Efficiency for Proximal Joint. . . . . . . . . . . 26

2.3 Performance Efficiency for Distal Joint. . . . . . . . . . . . 27

2.4 Selected design parameters of actuation system. . . . . . . . 38

2.5 Maximum required actuator torques for each manoeuvre. . . . 38

3.1 List of measured geometric properties. . . . . . . . . . . . 48

5.1 Transfer function coefficients for Case 1 and Case 2. . . . . . 71

$6.1 \quad$ Description of selected reference signals. . . . . . . . . . . . . 94

6.2 Key scenarios of LP joint covered in this Chapter. . . . . . . . 96 


\section{List of Figures}

$2.1 \quad$ Single SPOT platform. . . . . . . . . . . . . . . 10

$2.2 \quad$ Selected capture cases and the corresponding grip configurations of the proposed gripper: Case A is a surface capture, Case $\mathrm{B}$ is the corner grip, and Case $\mathrm{C}$ is the semi-cooperative enveloping grasp. . . . . . . . . . . . . . . . . 11

$2.3 \quad$ Selected joint configuration for the gripper. . . . . . . . . 13

2.4 Effect of joint angle on the torque arm of the applied tension. 13

$2.5 \quad$ Simplified schematic of the joint geometry. . . . . . . . . . 14

$2.6 \quad$ Constraint definition of the proximal joint. . . . . . . . 16

2.7 Constraint definition of the distal joint. . . . . . . . . 17

$2.8 \quad$ Coordinate system mapped to distal joint for the proximal link edge constraint. . . . . . . . . . . . . . . . . . . 18

2.9 Demonstration of infinite optimal solutions on the distal joint. 20

2.10 Optimal line and constraints mapped to the $x_{1}-x_{2}$ coordinate frame. . . . . . . . . . . . . . . . . 21

2.11 Moment arm performance comparison of Case P1 and Case P2 compared against the optimal case as a function of joint angle.

2.12 Moment arm performance comparison of Case D1 and Case D2 compared against the optimal case as a function of joint angle.

2.13 Performance efficiency relative to optimal curve for Case P1 and Case P2. . . . . . . . . . . . . . . . . . . 25

2.14 Performance efficiency relative to optimal curve for Case D1 and Case D2. . . . . . . . . . . . . . . . . 26

2.15 Detumbling manoeuvre of target under GC-A. . . . . . 28

$2.16 \quad$ FBD of detumbling manoeuvre of target under GC-A . . . . 29

2.17 Push manoeuvre of the target in GC-B. . . . . . . . . 31

2.18 Pull manoeuvre of the target in GC-C. . . . . . . . . 32

2.19 Infinitesimal tendon element within sheath. . . . . . . . . 34 
$2.20 \quad$ Critical point within CSTM. . . . . . . . . . 35

$2.21 \quad$ Worst-case sheath routing through arm and gripper. . . . . 36

3.1 SPOT facilities: gravity offset surface and two free-floating plat-

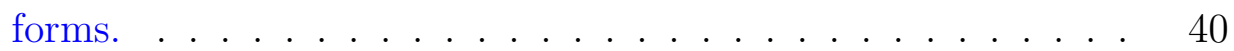

3.2 Gripper design. Exploded view (left) and assembled view (right). 41

$3.3 \quad$ Fully assembled gripper. . . . . . . . . . . . . . 42

3.4 Left: friction fit of sheath into the proximal link for the distal joint and torsion spring mounting. Right: friction fit of sheath into the base for the proximal joint. . . . . . . . . . . . . 43

3.5 Ferrule socket used to attach cable to the pin-off point. . . . . 43

3.6 Actuator bank design. Exploded view (left) and assembled view (right) . . . . . . . . . . . . . . . . . . 44 44

$3.7 \quad$ CSTM integrated into the actuator bank. . . . . . . . 44

$3.8 \quad$ Full assembly of the actuated gripper system. . . . . . . 45

$3.9 \quad$ Fully integrated gripper onto the SPOT platform. . . . . . 45

3.10 Location of LED markers on the SPOT platforms. . . . . . 46

3.11 Inverse kinematic model of the SPOT platform with arm+gripper. 47

3.12 Top level Simulink diagram to execute experiments. . . . . . 50

3.13 Main control logic to drive the experiment. . . . . . . . 50

$3.14 \quad$ Internal Dynamixel PID control logic [53]. . . . . . . . . 52

4.1 General control architecture for the CSTM driven gripper. . . 54

4.2 Decoupled hysteresis model and plant for non-linear system. . 60

4.3 Graphical representation of the non-linear transformation applied to the system input $\mathbf{u}(t) \ldots \ldots \ldots \ldots$. . . . . . 61

$4.4 \mathcal{L}_{1}$ control architecture for systems with uncertain input gain. 65

5.1 General control architecture for systems with uncertain input gain. . . . . . . . . . . . . . . . . 6 68

5.2 Simplified schematic of the actuation system in the testbed. . 69 
5.3 Multi-step response of the actuation system controlling the LP joint. . . . . . . . . . . . . . . . 70

5.4 Multi-step response of the Case 1 and Case 2 compared to experimental data. . . . . . . . . . . . . . . . . . 72

5.5 Absolute error of Case 1 and Case 2 with respect to experimental data. . . . . . . . . . . . . . . . . . 72

$5.6 \quad$ Implementation of Case 2 model into Simulink. . . . . . . . 73

5.7 Implementation of the CSTM model into Simulink. . . . . . . 74

5.8 Experimental data of the LP joint of Manoeuvre 1. . . . . . . 74

5.9 Propagation of CSTM parameters within the parameter estimation routine. . . . . . . . . . . . . . 75

5.10 Comparison of experimental and simulated data of the LP joint. 76

5.11 Simulated LP joint performance under no control. Top: actual and desired proximal link angular trajectory, bottom: trajectory tracking error, and right: hysteresis loop of transient phase. 77 5.12 Simulink diagram of the implemented PID controller. . . . . . 78

5.13 Simulated LP joint performance under PID control. Top: actual and desired proximal link angular trajectory, bottom: trajectory tracking error, and right: hysteresis loop of transient phase. . . . . . . . . . . . . . . . . 78

5.14 Algorithm implementation for the AB controller for the LP joint. 79

$5.15 \quad$ Effect of $k_{1}$ on closed-loop response. . . . . . . . . . 81

5.16 Effect of $k_{2}$ on closed-loop response. . . . . . . . . . . . 82

$5.17 \quad$ Effect of $\delta$ on closed-loop response. . . . . . . . . . . . 82

5.18 Effect of $\sigma$ on closed-loop response. . . . . . . . . . . 83

$5.19 \quad$ Effect of $\alpha$ on closed-loop response. . . . . . . . . . . 83

5.20 Simulated LP joint performance under AB control. Top: actual and desired proximal link angular trajectory, bottom: trajectory tracking error, and right: hysteresis loop of transient phase. 85

$5.21 \quad$ Modification to system architecture for $\mathcal{L}_{1}$ controller. . . . . . 86 
$5.22 \quad$ Simulink implementation of the $\mathcal{L}_{1}$ controller. . . . . . . . . . 87

5.23 Effect of $\Gamma$ on closed-loop response. . . . . . . . . . 90

5.24 Effect of $k$ on closed-loop response. . . . . . . . . . . . . . 90

5.25 Effect of $t_{s}$ on closed-loop response. . . . . . . . . . . 91

5.26 Effect of $\psi$ on closed-loop response. . . . . . . . . . . . . . 91

5.27 Simulated LP joint performance under $\mathcal{L}_{1}$ control. Top: actual and desired proximal link angular trajectory, bottom: trajectory tracking error, and right: hysteresis loop of transient phase. 93

6.1 LP joint performance in straight arm configuration under no control. Top: actual and desired proximal link angular trajectory, bottom: mean trajectory tracking error, and right: mean hysteresis loop of transient phase. . . . . . . . . . . . . . . 96

6.2 LP joint performance in bent arm configuration under no control. Top: actual and desired proximal link angular trajectory, bottom: mean trajectory tracking error, and right: mean hysteresis loop of transient phase. . . . . . . . . . . . .

6.3 LP joint performance with moving arm under no control. Top: actual and desired proximal link angular trajectory, bottom: mean trajectory tracking error, and right: mean hysteresis loop of transient phase. . . . . . . . . . . . . . .

6.4 LP joint performance in straight arm configuration under PID control. Top: actual and desired proximal link angular trajectory, bottom: mean trajectory tracking error, and right: mean hysteresis loop of transient phase. . . . . . . . . . . 100

6.5 LP joint performance in bent arm configuration under PID control. Top: actual and desired proximal link angular trajectory, bottom: mean trajectory tracking error, and right: mean hysteresis loop of transient phase. . . . . . . . . . . . . . 101 
6.6 LP joint performance with moving arm under PID control. Top: actual and desired proximal link angular trajectory, bottom: mean trajectory tracking error, and right: mean hysteresis loop of transient phase. . . . . . . . . . . . . . . . . 102

6.7 LP joint performance in straight arm configuration under AB control. Top: actual and desired proximal link angular trajectory, bottom: mean trajectory tracking error, and right: mean hysteresis loop of transient phase. . . . . . . . . . . . . . . 103

6.8 LP joint performance in bent arm configuration under AB control. Top: actual and desired proximal link angular trajectory, bottom: mean trajectory tracking error, and right: mean hysteresis loop of transient phase. . . . . . . . . . . . . . . . . . 104

6.9 LP joint performance with moving arm under AB control. Top: actual and desired proximal link angular trajectory, bottom: mean trajectory tracking error, and right: mean hysteresis loop of transient phase. . . . . . . . . . . . . . . . . 105

6.10 LP joint performance in straight arm configuration under $\mathcal{L}_{1}$ control. Top: actual and desired proximal link angular trajectory, bottom: mean trajectory tracking error, and right: mean hysteresis loop of transient phase. . . . . . . . . . . . . . 106

6.11 LP joint performance in bent arm configuration under $\mathcal{L}_{1}$ control. Top: actual and desired proximal link angular trajectory, bottom: mean trajectory tracking error, and right: mean hysteresis loop of transient phase. . . . . . . . . . . . . 107

6.12 LP joint performance with moving arm under $\mathcal{L}_{1}$ control. Top: actual and desired proximal link angular trajectory, bottom: mean trajectory tracking error, and right: mean hysteresis loop of transient phase. . . . . . . . . . . . . . . 108 
C.1 LP joint performance for the Sine Wave manoeuvre in a straight arm configuration under no control. Top: actual and desired proximal link angular trajectory, bottom: mean trajectory tracking error, and right: mean hysteresis loop of transient phase. . 157

C.2 LP joint performance for Manoeuvre 2 in a straight arm configuration under no control. Top: actual and desired proximal link angular trajectory, bottom: mean trajectory tracking error, and right: mean hysteresis loop of transient phase. . . . . . 158

C.3 LP joint performance for the Sine Wave manoeuvre in a bent arm configuration under no control. Top: actual and desired proximal link angular trajectory, bottom: mean trajectory tracking error, and right: mean hysteresis loop of transient phase. . 158

C.4 LP joint performance for Manoeuvre 2 in a bent arm configuration under no control. Top: actual and desired proximal link angular trajectory, bottom: mean trajectory tracking error, and right: mean hysteresis loop of transient phase. . . . . . . . .

C.5 LD joint performance for the Sine Wave manoeuvre in a straight arm configuration under no control. Top: actual and desired proximal link angular trajectory, bottom: mean trajectory tracking error, and right: mean hysteresis loop of transient phase. . 159

C.6 LD joint performance for Manoeuvre 1 in a straight arm configuration under no control. Top: actual and desired proximal link angular trajectory, bottom: mean trajectory tracking error, and right: mean hysteresis loop of transient phase. . . . . . . . 160

C.7 LD joint performance for Manoeuvre 2 in a straight arm configuration under no control. Top: actual and desired proximal link angular trajectory, bottom: mean trajectory tracking error, and right: mean hysteresis loop of transient phase. . . . . . . . 160 
C.8 LD joint performance for the Sine Wave manoeuvre in a bent arm configuration under no control. Top: actual and desired proximal link angular trajectory, bottom: mean trajectory tracking error, and right: mean hysteresis loop of transient phase. . 161

C.9 LD joint performance for Manoeuvre 1 in a straight arm configuration under no control. Top: actual and desired proximal link angular trajectory, bottom: mean trajectory tracking error, and right: mean hysteresis loop of transient phase. . . . . . . 161

C.10 LD joint performance for Manoeuvre 2 in a bent arm configuration under no control. Top: actual and desired proximal link angular trajectory, bottom: mean trajectory tracking error, and right: mean hysteresis loop of transient phase. . . . . . . . . . 162

C.11 LD joint performance with moving arm under no control. Top: actual and desired proximal link angular trajectory, bottom: mean trajectory tracking error, and right: mean hysteresis loop of transient phase. . . . . . . . . . . . . . . . 162

C.12 LP joint performance for the Sine Wave manoeuvre in a straight arm configuration under PID control. Top: actual and desired proximal link angular trajectory, bottom: mean trajectory tracking error, and right: mean hysteresis loop of transient phase. 163

C.13 LP joint performance for Manoeuvre 2 in a straight arm configuration under PID control. Top: actual and desired proximal link angular trajectory, bottom: mean trajectory tracking error, and right: mean hysteresis loop of transient phase. . . . . . . . 163

C.14 LP joint performance for the Sine Wave manoeuvre in a bent arm configuration under PID control. Top: actual and desired proximal link angular trajectory, bottom: mean trajectory tracking error, and right: mean hysteresis loop of transient phase.164 
C.15 LP joint performance for Manoeuvre 2 in a bent arm configuration under PID control. Top: actual and desired proximal link angular trajectory, bottom: mean trajectory tracking error, and right: mean hysteresis loop of transient phase. . . . . . . . . 164

C.16 LD joint performance for the Sine Wave manoeuvre in a straight arm configuration under PID control. Top: actual and desired proximal link angular trajectory, bottom: mean trajectory tracking error, and right: mean hysteresis loop of transient phase. 165

C.17 LD joint performance for Manoeuvre 1 in a straight arm configuration under PID control. Top: actual and desired proximal link angular trajectory, bottom: mean trajectory tracking error, and right: mean hysteresis loop of transient phase. . . . . . . 165

C.18 LD joint performance for Manoeuvre 2 in a straight arm configuration under PID control. Top: actual and desired proximal link angular trajectory, bottom: mean trajectory tracking error, and right: mean hysteresis loop of transient phase. . . . . . . 166

C.19 LD joint performance for the Sine Wave manoeuvre in a bent arm configuration under PID control. Top: actual and desired proximal link angular trajectory, bottom: mean trajectory tracking error, and right: mean hysteresis loop of transient phase.166

C.20 LD joint performance for Manoeuvre 1 in a straight arm configuration under PID control. Top: actual and desired proximal link angular trajectory, bottom: mean trajectory tracking error, and right: mean hysteresis loop of transient phase. . . . . . . 167

C.21 LD joint performance for Manoeuvre 2 in a bent arm configuration under PID control. Top: actual and desired proximal link angular trajectory, bottom: mean trajectory tracking error, and right: mean hysteresis loop of transient phase. . . . . . . . . 167 
C.22 LD joint performance with moving arm under PID control. Top: actual and desired proximal link angular trajectory, bottom: mean trajectory tracking error, and right: mean hysteresis loop of transient phase. . . . . . . . . . . . . . . . . 168

C.23 LP joint performance for the Sine Wave manoeuvre in a straight arm configuration under $\mathrm{AB}$ control. Top: actual and desired proximal link angular trajectory, bottom: mean trajectory tracking error, and right: mean hysteresis loop of transient phase. 168

C.24 LP joint performance for Manoeuvre 2 in a straight arm configuration under $\mathrm{AB}$ control. Top: actual and desired proximal link angular trajectory, bottom: mean trajectory tracking error, and right: mean hysteresis loop of transient phase. . . . . . . 169

C.25 LP joint performance for the Sine Wave manoeuvre in a bent arm configuration under $\mathrm{AB}$ control. Top: actual and desired proximal link angular trajectory, bottom: mean trajectory tracking error, and right: mean hysteresis loop of transient phase.169

C.26 LP joint performance for Manoeuvre 2 in a bent arm configuration under AB control. Top: actual and desired proximal link angular trajectory, bottom: mean trajectory tracking error, and right: mean hysteresis loop of transient phase. . . . . . . . . 170

C.27 LD joint performance for the Sine Wave manoeuvre in a straight arm configuration under $\mathrm{AB}$ control. Top: actual and desired proximal link angular trajectory, bottom: mean trajectory tracking error, and right: mean hysteresis loop of transient phase.170

C.28 LD joint performance for Manoeuvre 1 in a straight arm configuration under $\mathrm{AB}$ control. Top: actual and desired proximal link angular trajectory, bottom: mean trajectory tracking error, and right: mean hysteresis loop of transient phase. . . . . . . . 171 
C.29 LD joint performance for Manoeuvre 2 in a straight arm configuration under $\mathrm{AB}$ control. Top: actual and desired proximal link angular trajectory, bottom: mean trajectory tracking error, and right: mean hysteresis loop of transient phase. . . . . . . 171

C.30 LD joint performance for the Sine Wave manoeuvre in a bent arm configuration under $\mathrm{AB}$ control. Top: actual and desired proximal link angular trajectory, bottom: mean trajectory tracking error, and right: mean hysteresis loop of transient phase. 172

C.31 LD joint performance for Manoeuvre 1 in a straight arm configuration under $\mathrm{AB}$ control. Top: actual and desired proximal link angular trajectory, bottom: mean trajectory tracking error, and right: mean hysteresis loop of transient phase. . . . . . . 172

C.32 LD joint performance for Manoeuvre 2 in a bent arm configuration under $\mathrm{AB}$ control. Top: actual and desired proximal link angular trajectory, bottom: mean trajectory tracking error, and right: mean hysteresis loop of transient phase. . . . . . . . . . 173

C.33 LD joint performance with moving arm under AB control. Top: actual and desired proximal link angular trajectory, bottom: mean trajectory tracking error, and right: mean hysteresis loop of transient phase. . . . . . . . . . . . . . . . . 173

C.34 LP joint performance for the Sine Wave manoeuvre in a straight arm configuration under $\mathcal{L}_{1}$ control. Top: actual and desired proximal link angular trajectory, bottom: mean trajectory tracking error, and right: mean hysteresis loop of transient phase. . 174

C.35 LP joint performance for Manoeuvre 2 in a straight arm configuration under $\mathcal{L}_{1}$ control. Top: actual and desired proximal link angular trajectory, bottom: mean trajectory tracking error, and right: mean hysteresis loop of transient phase. . . . . . . . 174 
C.36 LP joint performance for the Sine Wave manoeuvre in a bent arm configuration under $\mathcal{L}_{1}$ control. Top: actual and desired proximal link angular trajectory, bottom: mean trajectory tracking error, and right: mean hysteresis loop of transient phase. . 175

C.37 LP joint performance for Manoeuvre 2 in a bent arm configuration under $\mathcal{L}_{1}$ control. Top: actual and desired proximal link angular trajectory, bottom: mean trajectory tracking error, and right: mean hysteresis loop of transient phase. . . . . . . . . 175

C.38 LD joint performance for the Sine Wave manoeuvre in a straight arm configuration under $\mathcal{L}_{1}$ control. Top: actual and desired proximal link angular trajectory, bottom: mean trajectory tracking error, and right: mean hysteresis loop of transient phase.

C.39 LD joint performance for Manoeuvre 1 in a straight arm configuration under $\mathcal{L}_{1}$ control. Top: actual and desired proximal link angular trajectory, bottom: mean trajectory tracking error, and right: mean hysteresis loop of transient phase. . . . . . . 176

C.40 LD joint performance for Manoeuvre 2 in a straight arm configuration under $\mathcal{L}_{1}$ control. Top: actual and desired proximal link angular trajectory, bottom: mean trajectory tracking error, and right: mean hysteresis loop of transient phase. . . . . . 177

C.41 LD joint performance for the Sine Wave manoeuvre in a bent arm configuration under $\mathcal{L}_{1}$ control. Top: actual and desired proximal link angular trajectory, bottom: mean trajectory tracking error, and right: mean hysteresis loop of transient phase. . 177

C.42 LD joint performance for Manoeuvre 1 in a straight arm configuration under $\mathcal{L}_{1}$ control. Top: actual and desired proximal link angular trajectory, bottom: mean trajectory tracking error, and right: mean hysteresis loop of transient phase. . . . . . . 178 
C.43 LD joint performance for Manoeuvre 2 in a bent arm configuration under $\mathcal{L}_{1}$ control. Top: actual and desired proximal link angular trajectory, bottom: mean trajectory tracking error, and right: mean hysteresis loop of transient phase. . . . . . . . . 178

C.44 LD joint performance with moving arm under $\mathcal{L}_{1}$ control. Top: actual and desired proximal link angular trajectory, bottom: mean trajectory tracking error, and right: mean hysteresis loop of transient phase. . . . . . . . . . . . . . . . . . 179 


\section{List of Symbols}

In this thesis, there is overlap in variables used based on the subject. Therefore, the list of variables will be divided by their first appearance in each chapter.

\section{Chapter 2 Symbols}

\begin{tabular}{|c|c|}
\hline$L_{0}$ & Span of the open gripper, $[\mathrm{m}]$ \\
\hline$\Lambda$ & Pin-off point of the sheath \\
\hline $\mathrm{P}$ & Pin-off point of the tendon \\
\hline $\mathbf{X}$ & Decision variable array of for the sheath and cable placement $A$ and $B,[\mathrm{~m}]$ \\
\hline$x_{1}$ & Horizontal coordinate of sheath termination point, $[\mathrm{m}]$ \\
\hline$x_{2}$ & Vertical coordinate of sheath termination point, $[\mathrm{m}]$ \\
\hline$x_{3}$ & Horizontal coordinate of cable termination point, $[\mathrm{m}]$ \\
\hline$x_{4}$ & Vertical coordinate of cable termination point, $[\mathrm{m}]$ \\
\hline$\theta$ & Joint angle, $[\mathrm{rad}]$ \\
\hline$r_{t}(\mathbf{x}, \theta)$ & Effective moment arm of the cable at a joint, $[\mathrm{m}]$ \\
\hline$f_{i}(\boldsymbol{x})$ & Inequality constraints on $\mathbf{x}$ where $i=1, \ldots, m$, for a scalar $b_{i}[\mathrm{~m}]$ \\
\hline$g_{j}(\boldsymbol{x})$ & Equality constraints on $\mathbf{x}$ where $j=1, \ldots, n$, for a scalar $c_{j}[\mathrm{~m}]$ \\
\hline$L_{e}(\mathbf{x}, \theta)$ & Length of exposed cable at the joint, $[\mathrm{m}]$ \\
\hline$h_{1}$ & Distance of point A from the joint, $[\mathrm{m}]$ \\
\hline$h_{0}$ & Distance of point B from the joint, $[\mathrm{m}]$ \\
\hline$\alpha_{1}, \alpha_{2}, \gamma$ & Geometric angles used in $r_{t}(\mathbf{x}, \theta),[\mathrm{rad}]$ \\
\hline d & Angle between exposed cable and $h_{1},[\mathrm{rad}]$ \\
\hline$R$ & Angle used in constraint formulation, [rad] \\
\hline d & Distance between edge constraint and edge, $[\mathrm{m}]$ \\
\hline & Geometric property of the gripper base, $[\mathrm{m}]$ \\
\hline$m_{1}$ & Slope of the optimal line, $[\mathrm{m} / \mathrm{m}]$ \\
\hline & $x_{2}$ intercept of the optimal line, $[\mathrm{m}]$ \\
\hline & Slope of the edge constraint, $[\mathrm{m} / \mathrm{m}]$ \\
\hline & $x_{2}$ intercept of the edge constraint, $[\mathrm{m}]$ \\
\hline
\end{tabular}




$\begin{array}{ll}a_{p} & \text { Maximum linear acceleration of target platform, }\left[\mathrm{m} / \mathrm{s}^{2}\right] \\ \alpha_{p} & \text { Maximum angular acceleration of target platform, }\left[\mathrm{rad} / \mathrm{s}^{2}\right] \\ I_{p} & \text { Mass moment of inertia of target platform, }\left[\mathrm{kg} \cdot \mathrm{m}^{2}\right] \\ \vec{l}_{1} & \text { Position of COM relative to proximal joint }(\mathrm{CG}-\mathrm{A}),[\mathrm{m}] \\ \vec{l}_{2} & \text { Position of COM relative to proximal joint }(\mathrm{GC}-\mathrm{B}),[\mathrm{m}] \\ \vec{l}_{3} & \text { Position of COM relative to proximal joint }(\mathrm{GC}-\mathrm{C}),[\mathrm{m}] \\ T & \text { Magnitude of torque, }[\mathrm{N} \cdot \mathrm{m}] \\ F & \text { Magnitude of force, }[\mathrm{N}] \\ d F_{T}(x) & \text { Infinitesimal cable tension, }[\mathrm{N}] \\ x & \text { Position along cable from input end, }[\mathrm{m}] \\ d x & \text { Infinitesimal length of cable element, }[\mathrm{m}] \\ d \gamma & \text { Infinitesimal arc angle of cable element, }[\mathrm{rad}] \\ R(x) & \text { Radius of cable, }[\mathrm{m}] \\ \mu_{s} & \text { Coefficient of static friction } \\ L & \text { Length of sheath, [m] } \\ L_{1} & \text { Critical point of cable-sheath transmission mechanism, [m] } \\ \Theta_{T} & \text { Total bend angle of the sheath, [rad] } \\ r_{p} & \text { Radius of winch pulley, }[\mathrm{m}] \\ \eta & \text { Scaling factor on stall torque }\end{array}$

\section{Subscript}

$\begin{array}{ll}b & \text { Base of the gripper } \\ p & \text { Proximal joint } \\ p_{1} & \text { Proximal end of proximal link } \\ p_{2} & \text { Distal end of proximal link } \\ d & \text { Distal joint } \\ \text { min } & \text { Minimum of variable } \\ \text { max } & \text { Maximum of variable } \\ \text { des } & \text { Desired value } \\ \text { opt } & \text { Optimal value }\end{array}$




$\begin{array}{ll}\text { temp } & \text { Temporary value } \\ s & \text { Spring } \\ c & \text { Cable } \\ I & \text { Due to inertial loads } \\ A, B, C & \text { For grip configuration A, B and C } \\ o & \text { Output end of cable-sheath transmission mechanism } \\ 0 & \text { Initial value } \\ s p & \text { Proximal joint spring } \\ s d & \text { Distal joint spring } \\ N & \text { Normal direction } \\ F & \text { Due to friction } \\ T & \text { Tension (for forces), or total (elsewhere) } \\ T_{\text {out }} & \text { Output tension } \\ T_{i} & \text { Input tension } \\ T_{0} & \text { Initial tension (or pre-tension) }\end{array}$

\section{Chapter 3 Symbols}

$\begin{array}{ll}\theta & \text { Joint angle, }[\mathrm{rad}] \\ l x, y) & \text { Inertial coordinates of a single point, }[\mathrm{m}] \\ \beta & \text { Constant geometric parameter (length), [m] } \\ \Delta l_{p} & \text { Constant geometric parameter (angular), [rad] } \\ \Delta l_{j} & \text { Change in cable displacement at the winch pulley, [m] } \\ r_{p} & \text { Change in cable displacement at the joint, }[\mathrm{m}] \\ r_{c} & \text { Radius of winch pulley, }[\mathrm{m}] \\ \theta_{m} & \text { Radius of cable, [m] } \\ \theta_{j} & \text { Angular position of the winch pulley, }[\mathrm{rad}] \\ K_{P}, K_{I}, K_{D} & \text { Point angle, [rad] }\end{array}$

Subscript

xxiv 
0-11 Number in order from shoulder joint

$S \quad$ Shoulder joint

E $\quad$ Elbow joint

$W \quad$ Wrist joint

$L P \quad$ Left proximal joint

$R P \quad$ Right proximal joint

$L D \quad$ Left distal joint

$R D \quad$ Right distal joint

min Minimum of variable

$\max \quad$ Maximum of variable

Chapter 4 Symbols

$\mathbf{x}_{i}$

$\theta_{i}$

$\dot{\theta}_{i}$

$\mathbf{u}(t)$

$\mathbf{r}(t)$

$\mathbf{x}(t)$

$\mathrm{A}, \mathrm{B}, \mathrm{C}$

$n$

$m$

$\boldsymbol{\omega}(t)$

$\boldsymbol{\sigma}_{d}(t)$

$\gamma(t)$

$\boldsymbol{\sigma}_{u}(t)$

$\boldsymbol{\sigma}(t)$

$\Upsilon_{0}$

$\Delta_{o}$

$k_{g}$

$\boldsymbol{\theta}(t)$
State array of joint $i$

Angle of joint $i,[\mathrm{rad}]$

Time derivative of $\theta_{i}$ at joint $i,[\mathrm{rad}]$

Control signal, [rad]

Reference signal, [rad]

Generalized state array

State space matrices

Number of states

Number of control inputs

Transformed control signal, [rad]

System disturbances, [rad]

Scaling factor in hysteresis transformation

Offset factor in hysteresis transformation, [rad]

Combined offset factor, [rad]

Set of $\gamma(t)$

Boundary on $\boldsymbol{\sigma}(t),[\mathrm{rad}]$

Scaling parameter used in $\mathcal{L}_{1}$ control

Unknown state gain uncertainties 


$\begin{array}{ll}\boldsymbol{\Theta} & \text { Set of } \boldsymbol{\theta}(t) \\ \dot{\hat{\mathbf{x}}}(t) & \text { Estimate of state array } \dot{\mathbf{x}}(t),[\mathrm{rad}] \\ \tilde{\mathbf{x}}(t) & \text { Parametric state estimation error, [rad] } \\ \Gamma & \text { Learning rate of CMAC and } \mathcal{L}_{1} \text { controller } \\ \dot{\hat{\gamma}}(t), \dot{\hat{\boldsymbol{\theta}}}(t), \dot{\hat{\boldsymbol{\sigma}}}(t) & \text { Adaptive laws for the } \mathcal{L}_{1} \text { controller } \\ \mathbf{P} & \text { Lyapunov solution matrix } \\ \mathbf{Q} & \text { Positive matrix used to find } \mathbf{P} \\ \boldsymbol{\eta}(t) & \text { Sum of system inputs, [rad] } \\ \mathbf{u}_{n}(t) & \text { Nominal CMAC controller } \\ \hat{\boldsymbol{\eta}}(t) & \text { Estimate of } \boldsymbol{\eta}(t),[\mathrm{rad}] \\ \lambda_{\min }(\mathbf{P}) & \text { Minimum eigenvalue of } \mathbf{P} \\ \bar{\theta}_{\text {max }} & \text { Scaled squared sum of all values of } \boldsymbol{\theta}(t) \\ \hat{\boldsymbol{\eta}}(s) & \text { Laplace transform of } \hat{\boldsymbol{\eta}}(t) \\ \mathbf{R}(s) & \text { Laplace transform of reference signal } \\ \mathbf{U}(s) & \text { Laplace transform of } \mathcal{L}_{1} \text { controller } \\ k & \text { Scalar gain on } \mathcal{L}_{1} \text { controller } \\ D(s) & \text { Filter used by } \mathcal{L}_{1} \text { controller } \\ C(s) & \text { Closed loop filter used by } \mathcal{L}_{1} \text { controller } \\ G(s), H(s) & \text { Transfer functions } \\ L & \text { The 1 norm of } \boldsymbol{\theta}(t) \\ x_{i n} & \text { Positional state at input of CSTM, [rad] or }[\mathrm{m}] \\ x_{\text {out }} & \text { Positional state at output of CSTM, [rad] or }[\mathrm{m}] \\ \alpha_{x}\left(\dot{x}_{i n}(t)\right) & \text { Smooth transition function in CSTM model } \\ \zeta(t) & \text { Internal state of CSTM model, [rad] or }[\mathrm{m}] \\ \alpha_{1}, \alpha_{2}>0, A, \nu, \delta & \text { Parameters in CSTM model } \\ d(t) & \text { Disturbance on internal state } \zeta(t),[\mathrm{rad}] \\ \Phi(t) & \text { Output of controlled CSTM, [rad] } \\ \Delta_{n}(t), \Delta_{n}^{*}(t) & \text { Perturbed internal state and its bound, [rad] } \\ x_{r}(t) & \text { Reference position, [rad] } \\ e_{r}(t) & \text { Error between output } \Phi(t) \text { and } x_{r}(t),[\mathrm{rad}] \\ & \end{array}$




$\begin{array}{ll}n(t) & \text { Cumulative integral of error, [rad } \cdot \mathrm{s}] \\ \omega(t), \dot{\omega}(t) & \text { Combination of error and its integral } \\ \alpha & \text { Scaling factor } \\ u_{1}(t), u_{2}(t) & \text { Decoupled controllers, [rad] } \\ \epsilon & \text { Smoothing factor in hyperbolic tan function } \\ \chi_{1}, \chi_{2} & \text { Inverse of } \alpha_{1} \text { and } \alpha_{2} \\ \hat{\chi}_{1}(t), \hat{\chi}_{2}(t), \hat{\Delta}_{n}^{*}(t) & \text { Adaptive estimates in AB controller } \\ \bar{u}_{1}(t), \bar{u}_{2}(t) & \text { Virtual controllers, [rad] } \\ k_{1}, k_{2} & \text { Proportional gains used in AB controller } \\ \delta_{1}, \delta_{2}, \delta_{3} & \text { Adaptation rates in AB controller } \\ \sigma_{1}, \sigma_{2}, \sigma_{3} & \text { Adaptive parameter limiter }\end{array}$

\section{Chapter 5 Symbols}

$\begin{array}{ll}R^{2} & \text { Coefficient of determination } \\ A(s) & \text { General transfer function } \\ b_{0}, b_{1}, b_{2} & \text { Numerator coefficients of general transfer function } \\ a_{0}, \ldots, a_{5} & \text { Denominator coefficients of general transfer function } \\ K_{p}, K_{i}, K_{d} & \text { PID controller gains for gripper } \\ t_{s} & \text { Settling time of companion model in } \mathcal{L}_{1} \text { control, [s] } \\ \mathbf{G}_{p}(s) & \text { Transfer function of companion system } \\ K & \text { Static gain on the companion system } \\ \omega_{n} & \text { Natural frequency of companion system } \\ \zeta & \text { Damping ratio of companion system } \\ \psi & \text { Filter coefficient in } \mathcal{L}_{1} \text { controller } \\ f(z) & \text { Discrete time low-pass filter applied in AB controller } \\ \delta & \text { Common AB controller adaptation rate } \\ \sigma & \text { Common AB controller adaptation limiter }\end{array}$




\section{List of Acronyms}

\begin{tabular}{|c|c|}
\hline $\mathrm{AB}$ & Indirect Adaptive Bouc-Wen controller \\
\hline $\mathrm{COM}$ & Center of Mass \\
\hline COTS & Commercial Off-The-Shelf \\
\hline $\mathrm{CNC}$ & Computer Numerical Control \\
\hline $\mathrm{CSA}$ & Canadian Space Agency \\
\hline CSTM & Cable Sheath Transmission Mechanism \\
\hline DARPA & Defense Advanced Research Projects Agency \\
\hline $\mathrm{DC}$ & Direct Current \\
\hline $\mathrm{DOF}$ & Degrees of Freedom \\
\hline DEMES & Dielectric Elastomer Minimum Energy Structures \\
\hline $\mathrm{EE}$ & End-Effector \\
\hline $\mathrm{ESA}$ & European Space Agency \\
\hline FBD & Free-Body Diagram \\
\hline FREND & Front-end Robotics Enabling Near-term Demonstration \\
\hline GC-A, GC-B, GC-C & Grip Configuration $\mathrm{A}, \mathrm{B}$, and $\mathrm{C}$ \\
\hline GEO & Geostationary Equatorial Orbit \\
\hline ID & Inner Diameter \\
\hline JAXA & Japan Aerospace Exploration Agency \\
\hline $\mathrm{KKT}$ & Karush-Kuhn-Tucker \\
\hline $\mathrm{LD}$ & Left-Distal joint \\
\hline LED & Light Emitting Diode \\
\hline LEE & Latching End-Effector \\
\hline LEO & Low Earth Orbit \\
\hline LP & Left-Proximal joint \\
\hline MATLAB & Matrix Laboratory \\
\hline MDA & MacDonald, Dettwiler and Associates \\
\hline MSE & Mean Squared Error \\
\hline
\end{tabular}




$\begin{array}{ll}\text { NASA } & \text { National Aeronautics and Space Administration } \\ \text { NRL } & \text { US Naval Research Laboratory } \\ \text { OD } & \text { Outer Diameter } \\ \text { OE } & \text { Orbital Express } \\ \text { PID } & \text { Proportional-Integral-Derivative controller } \\ \text { PTFE } & \text { Polytetrafluoroethene } \\ \text { RD } & \text { Right-Distal joint } \\ \text { RP } & \text { Right-Proximal joint } \\ \text { RP3 } & \text { Raspberry Pi 3 } \\ \text { RRM } & \text { Remote Refueling Mission } \\ \text { SISO } & \text { Single-Input Single-Output } \\ \text { SPOT } & \text { Spacecraft Proximity Operations Test-bed } \\ \text { SQP } & \text { Sequential Quadratic Programming } \\ \text { SRCL } & \text { Spacecraft Robotics and Control Laboratory } \\ \text { SS304 } & \text { Stainless-Steel 304 } \\ \text { SSRMS } & \text { Space Station Remote Manipulator System }\end{array}$

xxix 


\section{Chapter 1}

\section{Introduction}

\subsection{Motivation}

As low Earth orbits continue to be populated, there is an ever-increasing risk of collision between active spacecraft and orbital debris. In fact, there are nearly 19 000 catalogued objects over $10 \mathrm{~cm}$ in diameter that populate the orbital environment [1], including spent upper stages, fragmented debris, and non-operational spacecraft. Kessler demonstrated that this number will continue to grow exponentially as per the Kessler syndrome, which can be described as the perpetuated fragmentation of orbital objects through cascading collisions [2]. Even with no new launches, some models predict that the debris density will increase by a factor of approximately 2.4 in low Earth orbit (LEO) within a 200 year period [3]. If no action is taken, the future of spacecraft operations will undoubtedly be negatively impacted by higher costs and potential loss of entire missions [4].

Despite these alarming results, the European Space Agency (ESA) reported that removing as little as five large objects per year from the orbital environment would mitigate this unstable growth [5]. This can be accomplished through active debris removal missions, or by performing on-orbit servicing missions to extend the operation of existing satellites, thereby reducing the frequency of spacecraft launches. As a result, several space agencies (NASA, ESA, CSA and JAXA) are currently working on solutions to these problems to prevent the orbital environment from becoming inaccessible.

\subsection{Problem Statement}

The capture of an inactive satellite in orbit poses several challenges, as the target is considered "non-cooperative". Since the target may not be under its own control, 
or have a prescribed capture interface on the target, the servicing spacecraft must perform a capture manoeuvre which can overcome these problems. The preferred solution to this problem would be to employ a capture mechanism to the servicing craft that can accommodate a wide number of target geometries to ensure a rigid capture is made. Several capture strategies exist which have been proven to perform well under prescribed operating conditions, however, it becomes increasingly challenging to ensure that the servicing manipulator can perform unexpected tasks as they arise during the mission. In response to these challenges, this thesis is concerned with the preliminary development of a novel capture strategy to broaden utility in the context of de-orbiting manoeuvres and active servicing missions.

\subsection{Previous Work}

An extensive amount of research has been conducted in the field of capture strategies for space debris removal and active servicing missions. This section is dedicated to providing background for the reader by reviewing relevant works on the topic of this thesis. For reasons that will be further illustrated by the thesis objectives (Section 1.4), this literature review will be divided into two distinct topics: on-orbit capture mechanisms, and tendon driven manipulators.

\subsubsection{On-Orbit Capture Mechanisms}

There are numerous proposed solutions to addressing the orbital capture problem. Generally, this problem is divided into two primary subsets; flexible capture, and rigid capture of the target. [6,7]. In terms of flexible capturing methods it is generally accepted that a tethered connection is the most effective technology [8-11]. To connect the tether to the target, the most popular solutions include: equipping the tether with a flexible net to envelop the target [12], piercing the target with barbed harpoons [13], or by an active continuum capture device which envelops the target [14]. Despite its advantages, these flexible capture strategies cannot perform any precise manipulation tasks, and can therefore not be applied to servicing missions.

To enable manipulation tasks for on-orbit servicing missions and orbital debris removal, a rigid connection between the servicing craft and target is often preferred. 
Since a rigid connection can only be accomplished with a robotic manipulator, it is this subset of capture devices that are of key interest. In general, these robotic capture mechanisms can be applied to two mission secnarios: cooperative capture, and non-cooperative capture. Cooperative targets are under full control and have a predefined capture interface for the servicing craft to latch on to. Examples of flight-proven cooperative capture interfaces include the advanced robotics hand onboard the ETSVII space robot system [15], the widely known latching end-effector (LEE) currently used on the Space Station Remote Manipulator System (SSRMS) [16], the manipulation tools used on the Canadian Special Purpose Dextrous Manipulator robot during the Robotic Refueling mission (RRM) [17], and the orbital-express (OE) capture mechanism $[18,19]$. Since this thesis is interested in the capture of non-cooperative spacecraft or debris, which means the target will have no predefined capture interface, standard cooperative capture solutions such as these are unfortunately infeasible.

With non-cooperative targets, however, the object of interest is under no control of its own and does not have a predefined capture interface. To accomplish the capture of non-cooperative targets, several robotic strategies have been considered. The Phoenix program is an initiative developed by the Defense Advanced Research Projects Agency (DARPA) and US Naval Research Laboratory (NRL) to test technologies and strategies to validate their applicability for servicing missions, such as repair decommissioned spacecraft or debris removal in geostationary equatorial orbit (GEO) [20]. One example of an on-orbit demonstration is the testing of the Front-end Robotics Enabling Near-term Demonstration (FREND) arm equipped with a basic clamping capture tool to perform a robotic assembly of external payload, and a series of dexterous manipulation tasks [21]. Additionally, ESAs e.Deorbit mission plans to launch several debris removal technologies; one of which being a clamp-type robotic gripper mechanism [22]. Another example of a robotic clamping mechanism is the Launch Adapter Ring Capture Tool developed by MacDonald, Dettwiler and Associates (MDA); which employs a robotic clamping tool which latches to the launch adapter ring of unprepared targets [23]. While this method is suitable for targets that possess a launch adapter ring, it cannot perform a rigid capture if this interface does not exist. Another approach to robotic capture is to envelop the target with a 
continuum manipulator. An example of this technology is the "tentacle" type gripper developed for the CleanSpace One mission. Equipped with four dielectric elastomer minimum energy structures (DEMES) acting as the links, this mechanism is designed to envelop a nano-scale satellite by entirely enveloping the target [24]. This concept, though lightweight and universal, is unscalable to larger spacecraft due to its low rigidity and retention force limits.

A concept exists for capturing spacecraft by employing synthetic gecko adhesives to form an adhesive bond to the surface of the target [25], where it has been demonstrated to successfully perform a rigid capture on a free-floating target in a zero-G environment [26]. The key enabling technology that allows this form of capture is the nano-scale fibrillar adhesives that have been inspired by the tokay gecko. This technology is highly customizable, and can be designed to be switch-able, pre-load dependant, and can perform well on virtually any surface; even in the orbital environment [27-30]. To date, however, there has not been a concept proposed that can perform a rigid capture for a universally diverse target geometry.

\subsubsection{Tendon Driven Manipulators}

A tendon driven manipulator is a robotic device in which the joints are controlled by cables in tension connected to actuators located distally from the joints [31-33]. There are two main ways in which the tendons can be transmitted from the actuators to the joints. The first of which being a pulley routing system, whereby the tendon (or driving cable) is routed through a series of pulleys [34,35]. This method offers reduced transmission losses at the expense of greatly increasing the complexity of the manipulator. The other form of tendon transmission is the use of a cable-sheath transmission mechanism (CSTM), where the driving tendons are routed through a flexible conduit from the actuating mechanism to the joint [36-38]. The CSTM offers several benefits over the pulley configuration by reducing mass, volume, and complex-

ity, while increasing its flexibility. The main issue with the CSTM is that it introduces hysteretic friction non-linearities that vary with the configuration of the sheath.

There have been numerous models proposed to identify and mitigate these nonlinearities. Kaneko et al. $[38,39]$ was among the first researchers to develop a CSTM 
model that was based on Coulomb friction and lumped mass theory. In this work, it was assumed that the CSTM maintains a fixed curvature and that the cable is always under sufficient pre-tension. Palli et al. [40,41] then extended this research by considering the effects of the Dahl friction model on the CSTM and proposed a compensation strategy based on tension feedback to mitigate the effects of friction. Palli et al. [42] further improved upon his work by developing an optimal control strategy which further improved the CSTM compensation. These strategies, however, do not account for the tracking error of the output since they rely on force feedback of the cable tension. Furthermore, as the number of tendon elements is increased to improve its accuracy, the computational requirements for the control algorithm are greatly increased. In response, Agrawal utilized a general piece-wise differential backlash model to develop an inverse-feed-forward control scheme that was used to effectively improve the position tracking error of the output [43]. Do et al. [44] then improved upon this work by developing a continuous model (a modification to the Bouc-Wen asymmetric model) to remove the issues that arose from discontinuities in the general model.

Unfortunately, all of these proposed strategies operate under the assumption that the system is in a fixed configuration, and that the parameters of the system are known for their respective models. In practice, this is an unrealistic assumption since the configuration of the sheath will change as the manipulatoris actuated. In response to this challenge, Do et al. $[45,46]$ proposed an adaptive control strategy which utilizes the model developed in their previous work. This control strategy has been shown to yield among the best results for position tracking in robotic CSTM systems while having the capability of operating under dynamic conditions. A downside to this form of adaptive control is that it is indirect; implying that it still requires the form of the system dynamics model.

Conversely, direct adaptive control methods do not require explicit parameter identification, and are well-suited for a wide class of multiple-input multiple-output systems subjected to significant uncertainties and disturbances [47]. As such, a direct adaptive controller would be preferred for the control of a time varying system with little or no prior knowledge of the plant. To the best of the author's knowledge, there 
have been no direct adaptive control strategies applied to a CSTM.

\subsection{Thesis Objectives}

This work examines the design and direct adaptive control of a novel capture mechanism to perform a rigid capture on a non-cooperative target for a number of capture interfaces. The proposed solution consists of the design of a tendon-driven robotic gripper that has the capability to employ a synthetic gecko-adhesive contact interface on the links to create a hybridized capture strategy of the solutions outlined in Section 1.3.1. The advantage of such manipulator would allow a wider range of capture interfaces, and reduce the overall end-effector (EE) mass by distally locating the actuators from the joints.

To maintain an achievable scope, the design and implementation of an experimental prototype will be developed for use on the Spacecraft Proximity Operations Test-bed (SPOT) at Carleton University's Spacecraft Robotics and Control Laboratory (SRCL). Much like the design constraints that would exist for an actual mission, this mechanism must have a small volumetric profile, be lightweight, and have robust performance. Thus, this thesis is concerned with the optimal design and direct adaptive control of a lightweight robotic capture mechanism for the future investigation of its suitability on the capture of non-cooperative targets. Specifically, a direct adaptive control strategy based on the recently-developed $\mathcal{L}_{1}$ adaptive control theory is proposed.

The performance of the gripper under the $\mathcal{L}_{1}$ adaptive control scheme will be compared against several other control cases: no control, PID control, and the indirect adaptive strategy proposed by Do et al. [46]. The performance of these controllers will be investigated in both a simulated environment under a brief sensitivity analysis, and in experiment under a number of CSTM configurations.

\subsection{Contributions}

The contributions of this work to the field of spacecraft robotics and control include:

- A novel capture mechanism that has not been proposed for the purposes of 
non-cooperative target capture on-orbit.

- An optimal approach to designing the joint configurations to maximize joint torque transmission in the expected operating configurations.

- The development and implementation of a detachable symmetric two-link gripper for the laboratory facilities that can be used to perform various manipulation tasks.

- The implementation of an existing indirect adaptive controller that has only been used in a single isolated CSTM testbed into a functional multi-link system.

- The implementation of a direct $\mathcal{L}_{1}$ adaptive controller into a system actuated via CSTM.

- A brief sensitivity study to provide a qualitative method for tuning the two selected adaptive controllers.

- An experimental validation of the positional tracking performance of the gripper joints under the direct and indirect adaptive control methods.

\subsection{Organization}

This thesis is organized as follows:

The design of the proposed capture mechanism is provided in Chapter 2: End Effector Design, where the design requirements and overall concept will be introduced. Following this, the optimal joint configuration will be covered, along with the design of the actuation system to ensure compliance with the stated requirements.

Chapter 3: Experimental Facilities will cover the contributions that this work has made to the experimental facilities used by the SRCL. Specifically, this chapter covers the physical assembly of the gripper and its integration with the SPOT, the instrumentation and measurement system used to acquire state feedback from the gripper links, and a high-level view at the driving software used to control the experiments.

With the physical system identified, the control strategies used to actuate the gripper will be highlighted in Chapter 4: Adaptive Control Strategies for CSTM. In 
this chapter, a description of the experimental system is given with context to the control problem, followed by the theory of the selected control algorithms; the indirect adaptive controller proposed by Do et al. [45,46], and the $\mathcal{L}_{1}$ controller.

To ensure that the control algorithms are indeed suitable for the application prior to the experiments, Chapter 5: Control Simulations and Tuning constructs a simulated environment for the purposes of preliminarily evaluating the controller performance. After modelling the experimental plant through parametric estimation techniques, each selected control case (no control, PID control, indirect control by Do. et al. $[45,46]$, and $\mathcal{L}_{1}$ control) is then programmed in the MATLAB/Simulink environment to be easily transferred to the experimental testbed for later use. A brief sensitivity analysis is then given to provide a qualitative insight to tuning the adaptive controllers.

After the controllers have been programmed and implemented into the full experiment, the experimental results of the full articulated gripper are presented in Chapter 6: Experimental Results and Discussion. Due to the high number of selected cases, this chapter focuses on a single joint of the gripper and compares the performance across several CSTM configurations under static and dynamic conditions for each of the four control scenarios. All supplementary data is included in Appendix C.

Finally, Chapter 7: Conclusion ends this thesis with some conclusionary statements, a comment on the practicality of the proposed techniques in the context of spaceflight application, and suggestions on future work. 


\section{Chapter 2}

\section{End Effector Design}

As it was mentioned in Section 1.4, the capture mechanism proposed is a tendondriven robotic gripper that can be equipped with a synthetic gecko adhesive contact interface. Since this is a novel concept in the context of non-cooperative target capture of free-floating bodies, there are several design considerations that must be investigated to ensure that the system will operate as desired. Thus, this chapter is dedicated to outlining the details of these design considerations, among which include the selection of the gripper configuration based on requirements in Section 2.1, the design and optimization of the joints in Section 2.2, and the design of the CSTM and associated actuation hardware in Section 2.3. The final design and assembly of the gripper will be covered in Chapter 3, alongside an overview of the experimental facility.

\subsection{High Level Concept}

Prior to pursuing the detailed design of the robotic capture mechanism, the overall objectives and requirements for the gripper must first be established. As it was outlined previously in Section 1.4, the gripper considered in this work will be as a prototype for the SPOT platforms at the Carleton University SRCL. The SPOT platforms are a $30 \times 30 \times 30 \mathrm{~cm}$ free-floating platform which operate on a gravity offset table via planar air-bearings. The platforms are free to translate in Cartesian $x-y$ coordinates, while its attitude is defined about the $z$ axis normal to the table. A single SPOT platform can be seen in Fig. 2.1; for more details on the SRCL facility, please refer to Chapter 3.

Since the gripper must be compatible with these platforms, there are several constraints placed on the design. First and foremost, the gripper must be able to operate within the capabilities of the facility (see Section 3.1 for details). This means that the 


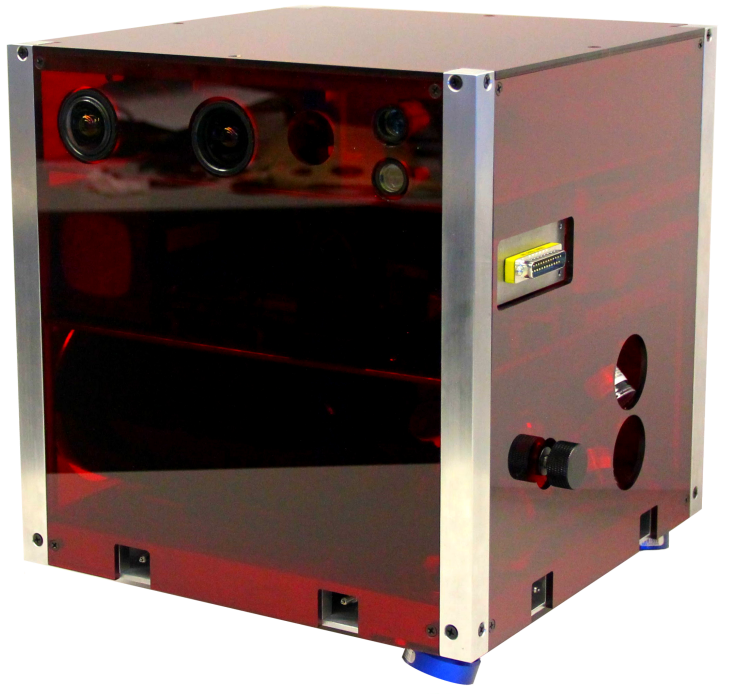

Figure 2.1: Single SPOT platform.

servicing and target platforms are predefined, imposing several hard constraints on the design of the gripper. The primary requirement is that the gripper must operate exclusively in a single plane. This immediately implies that the gripper will have revolute joints perpendicular to the operating plane. Secondly, based on the nature of the experimental platforms, it was determined that the capture mechanism must be able to perform the following capture manoeuvres on the target in future experiments: a flat surface capture utilizing the synthetic gecko adhesives (once acquired), a non-enveloping grasp on the corner of the target, and an enveloping grasp on a semi-cooperative capture handle (interchangeable). Additionally, the initial concept must be low in cost, mass, and complexity due to the nature of the project.

Several concepts were considered to achieve these key requirements, however, only the selected concept will be presented in this work. At its most fundamental level, the selected concept is a planar, fully actuated symmetric gripper with two-link phalanxes on each side. Due to the scale of the experiment, it is important that the open gripper does not exceed a length of $L_{0}<30 \mathrm{~cm}$ to ensure that the gripper can accommodate the proposed captures on the target as per Fig. 2.2. In this schematic, the captures are labelled as follows: the surface capture (capture case A) is achieved with grip configuration A (GC-A), the corner capture (capture case B) is achieved with GC-B, 
and the semi-cooperative capture (capture case C) is accomplished by GC-C. Note that the gecko adhesives must be fitted to the contact interface of each link to enable GC-A and GC-B.

Capture Case A

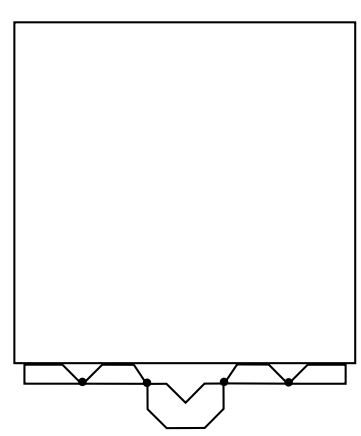

Capture Case B

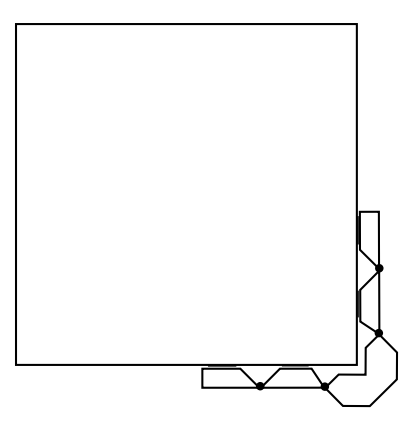

Capture Case C

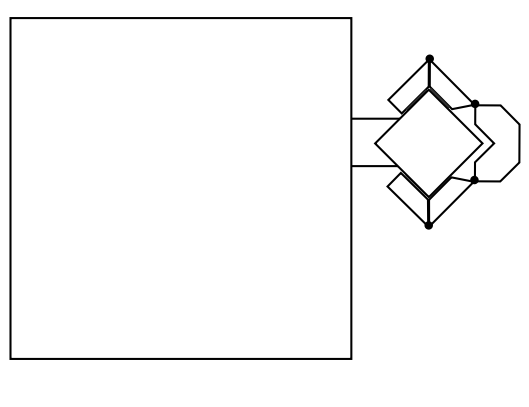

Figure 2.2: Selected capture cases and the corresponding grip configurations of the proposed gripper: Case A is a surface capture, Case B is the corner grip, and Case C is the semi-cooperative enveloping grasp.

\subsection{Joint Design}

With the general concept of the gripper in the previous section, the first step in the design of the end-effector is the design of the joints. This is necessary since all other systems of the EE, (i.e. actuation system, capture interface, control system, etc.,) are dependent upon the joint type and the CSTM connection points within the joint. Therefore, this section will cover the configuration selection process, followed by the optimal design of the CSTM within the joint itself.

\subsubsection{Joint Configuration Selection}

Based on the literature, there are several joint configurations that are possible for a tendon-driven manipulator. One common method is by fully rigidizing the joint by coupling an antagonistic pair of cables routed through independent sheaths $[32$, $34,35,38]$. This method has the advantage of ensuring that the joints are stiff, and that there is actuated control in both operating directions. However, this type of strategy doubles the amount of required hardware, is more complicated to install with the correct pre-tension, and the joint must have a pulley to function. Since the 
mechanism of interest only requires a clamping force in one direction, this solution would add cost, complexity, and mass to the project.

Conversely, there exists an open-ended design which employs a single antagonistic tendon in the direction of operation, and a spring to provide a restitution torque in the opening direction when the cable is slackened $[31,34,48]$. This method offers a great degree of flexibility in the design process as it simplifies a number of challenges associated with dual antagonistic methods. For instance, the use of a spring will ensure that the cable is in sufficient pretension at all times of its operation, while reducing the complexity and cost of the system. This configuration was selected due to the advantages that the open-ended single tendon design has in the context of this project.

An uncommon subset of this type of CSTM joint is one that does not employ a pulley at the joint. The use of the pulley is primarily used to maintain a constant torque arm at the joint, and to ensure that the tendon is routed properly. Since the volumetric form factor of the experiment is restricted, and the joints must be kept simplistic in their design, it was deemed most appropriate to select the joint type with no pulley. Thus, the selected joint configuration is highlighted by Fig. 2.3, where the key components are labelled.

\subsubsection{Optimized Joint Configuration}

Due to the nature of the selected CSTM joint type, the torque applied to the joint will be a function of both the cable tensions, and the joint angle itself. This is illustrated by Fig. 2.4, where two angles are shown for the same joint. 


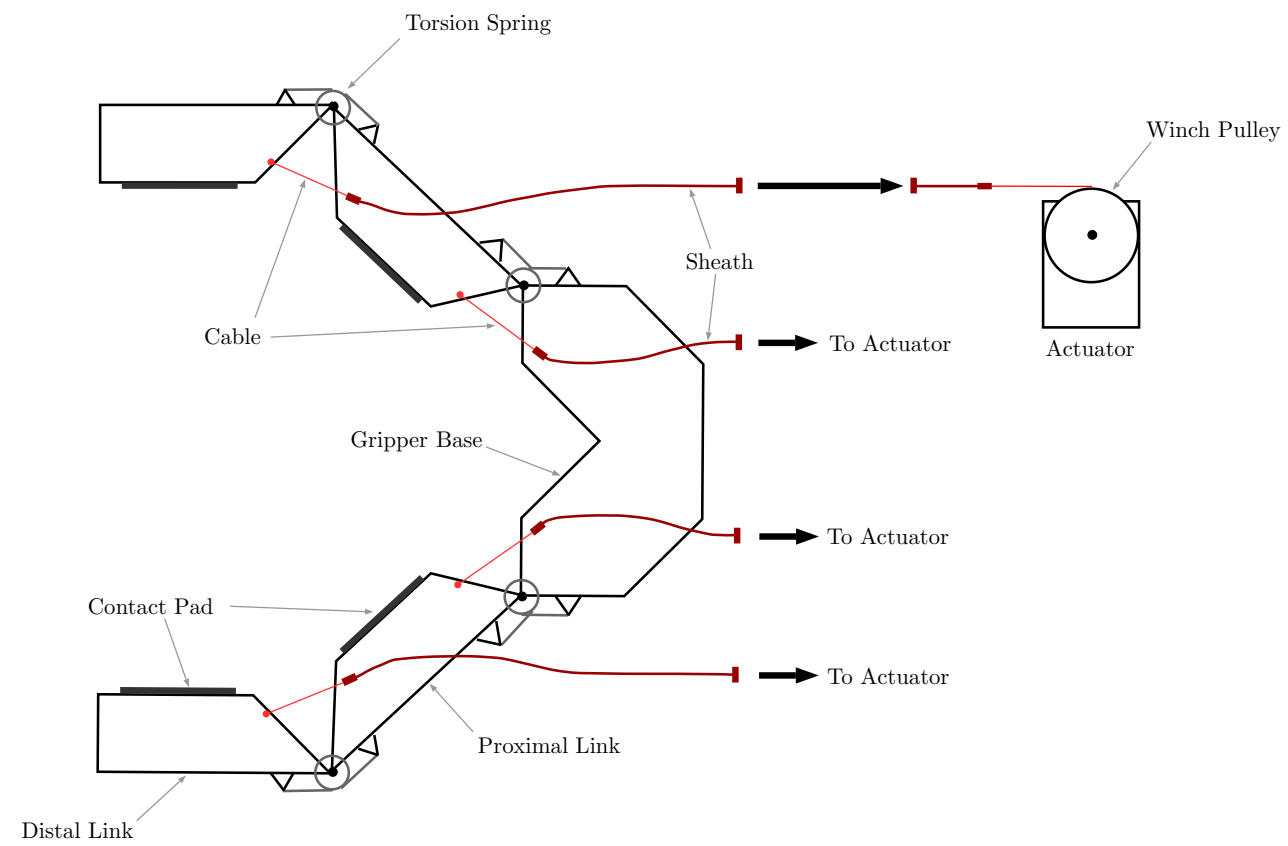

Figure 2.3: Selected joint configuration for the gripper.
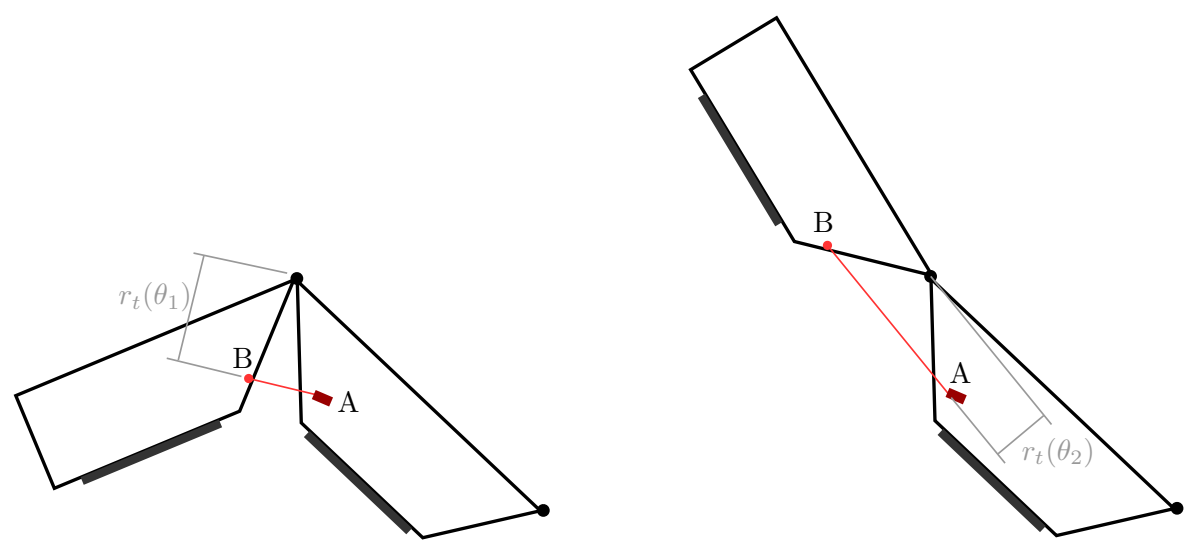

Figure 2.4: Effect of joint angle on the torque arm of the applied tension.

From the figure, it can be seen that the magnitude of the moment arm will vary as a function of the joint angle, ultimately resulting in a non-linear output torque profile. Additionally, there will be a joint angle which produces the maximum moment arm for fixed locations of both the sheath and tendon termination points within the links (points A and B respectively). Therefore, to ensure that the gripper will have a suitable performance for the proposed experiments, the geometry of the joint will be treated as an optimization problem, where the objective will be to select the location 
of $\mathrm{A}$ and $\mathrm{B}$ such that the moment arm is maximized for the grip configurations highlighted in Fig. 2.2.

\section{Objective Function Formulation}

Before the specifics of the optimization problem are determined, let us define the general form of the objective function and constraints.

$$
\begin{array}{cl}
\underset{\boldsymbol{x}}{\operatorname{maximize}} & r_{t}(\boldsymbol{x}, \theta) \\
\text { subject to } & f_{i}(\boldsymbol{x}) \leq b_{i}, i=1, \ldots, m \\
& g_{j}(\boldsymbol{x})=c_{j}, j=1, \ldots, n
\end{array}
$$

where $r_{t}(\boldsymbol{x}, \theta)$ represents the magnitude of the moment arm (the cost function), $\boldsymbol{x}$ represents the array of decision variables (placement of points $\mathrm{A}$ and $\mathrm{B}$ ), $\theta$ represents the joint angle, with the inequality constraints given by $f_{i}(\boldsymbol{x})$ and the equality constraints given by $g_{j}(\boldsymbol{x})$. To formulate the objective function and its constraints, let us first consider the simplified geometry of one of the joints; in this case, the distal joint is shown in Fig. 2.5.

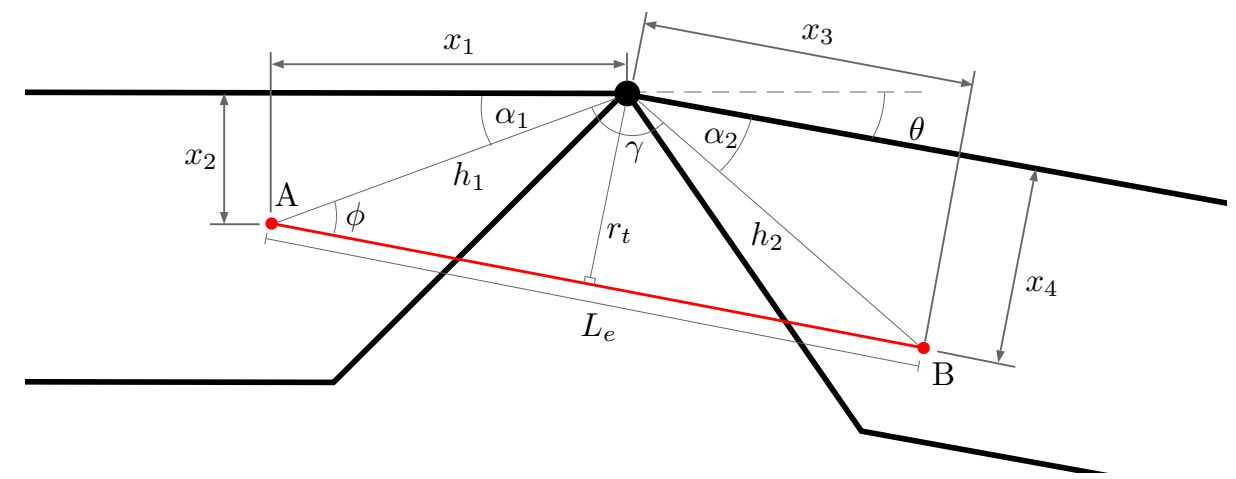

Figure 2.5: Simplified schematic of the joint geometry.

For a given joint angle $\theta$, the design parameters which affect the moment arm directly include the location of the sheath termination point on the proximal link $\left(x_{1}\right.$ and $\left.x_{2}\right)$, and the location of the tendon termination point on the distal link $\left(x_{3}\right.$ and $\left.x_{4}\right)$. Therefore, the decision variable array $\boldsymbol{x}$ will be defined as the following. 


$$
\boldsymbol{x}=\left[\begin{array}{l}
x_{1} \\
x_{2} \\
x_{3} \\
x_{4}
\end{array}\right]
$$

To find $r_{t}(\boldsymbol{x}, \theta)$, the height of the scalene triangle subtended between the joint and points $\mathrm{A}$ and $\mathrm{B}$ must be found. First, let us define the parameters $h_{1}, h_{2}, \alpha_{1}$, and $\alpha_{2}$ as a function of the decision variables.

$$
\begin{aligned}
h_{1} & =\sqrt{x_{1}^{2}+x_{2}^{2}} \\
h_{2} & =\sqrt{x_{3}^{2}+x_{4}^{2}} \\
\alpha_{1} & =\tan ^{-1}\left(\frac{x_{2}}{x_{1}}\right) \\
\alpha_{2} & =\tan ^{-1}\left(\frac{x_{4}}{x_{3}}\right)
\end{aligned}
$$

Additionally, the angle subtended between the line segments $h_{1}$ and $h_{2}$ can be found by the following expression.

$$
\gamma=\pi-\left(\theta+\alpha_{1}+\alpha_{2}\right)
$$

With Eq. (2.3) to (2.7) defined, the length of exposed tendon, $L_{e}$, can be found through the use of the cosine law.

$$
L_{e}(\boldsymbol{x}, \theta)=\sqrt{h_{1}^{2}+h_{2}^{2}+2 h_{1} h_{2} \cos \gamma}
$$

By making use of the law of sines, the angle $\phi$ can then be found.

$$
\phi=\sin ^{-1}\left(\frac{h_{2}}{L_{e} \sin \gamma}\right)
$$

Finally, the cost function $r_{t}(\boldsymbol{x}, \theta)$ can then be expressed in terms of $h_{1}$ and $\phi$.

$$
r_{t}(\boldsymbol{x}, \theta)=h_{1} \sin \phi
$$


Since Eq. (2.10) was formulated by solely considering the location of points A and $\mathrm{B}$ with respect to the joint axis, it can be used as the cost function for both the distal and proximal joint optimization problems. However, due to the physical differences between the two joints, each optimization problem will require a slightly different constraint formulation.

\section{Constraint Formulation}

Since the decision variables in the aforementioned optimization problem are entirely geometric, the constraints on these variables must also relate to the physical characteristics of the gripper. To formulate the constraints, consider the simplified geometry of both the proximal and distal joints in Fig. 2.6, and Fig. 2.7, respectively.

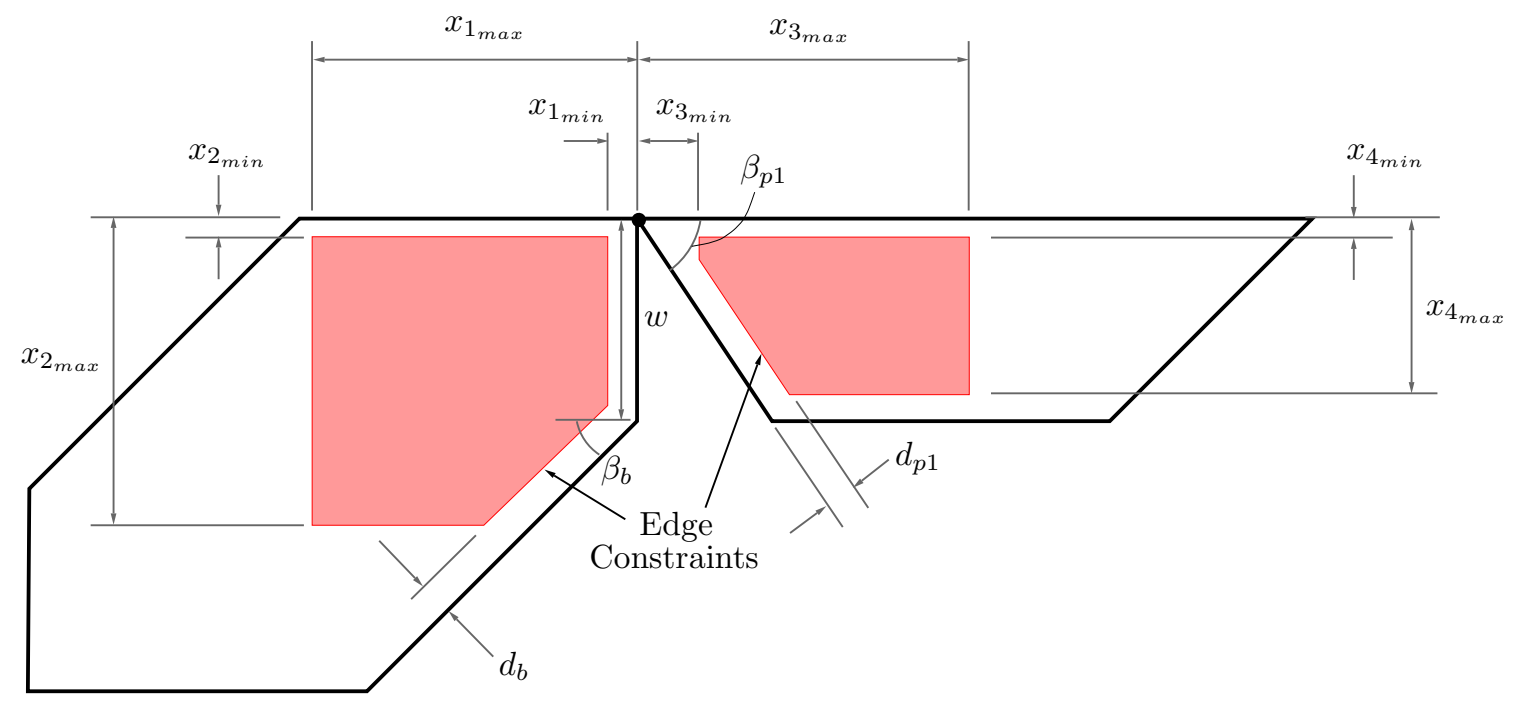

Figure 2.6: Constraint definition of the proximal joint.

From this geometric model, there are several constraints which can be derived. The first being the bounds on the decision variables, where the constraints can be formulated as per Eq. (2.11) to (2.14). Note that the actual values of $x_{i \min }$ and $x_{i \max }$ are different for the proximal and distal joints. 


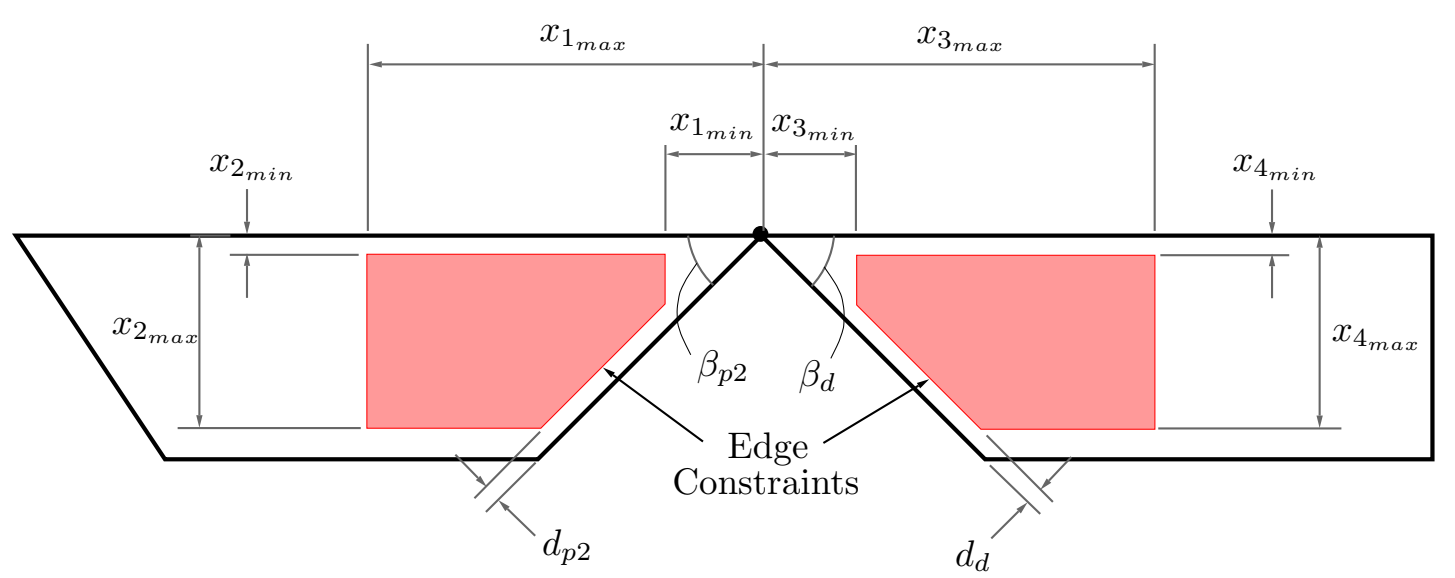

Figure 2.7: Constraint definition of the distal joint.

$$
\begin{aligned}
& x_{1 \text { min }} \leq x_{1} \leq x_{1 \max } \\
& x_{2 \min } \leq x_{2} \leq x_{2 \max } \\
& x_{3 \min } \leq x_{3} \leq x_{3 \max } \\
& x_{4 \min } \leq x_{4} \leq x_{4 \max }
\end{aligned}
$$

The edge constraints inequalities can be formulated in a way such that the points $\mathrm{A}$ and $\mathrm{B}$ must be at a distance $d_{i}$ from the angled edge. By fixing a coordinate system to the joint and aligning the principle axes in the direction of the decision variables, this constraint can be formulated as the linear combination of decision variables $x_{1}$ and $x_{2}$ as per Fig. 2.8, where the horizontal and vertical axes correspond to $x_{1}$ and $x_{2}$ respectively.

Since the distance $d_{i}$ and the angle $\beta_{i}$ are known based on design decisions, then the general equation of the line in Fig. 2.8 can be analytically expressed as per Eq. (2.15) below.

$$
x_{2}=x_{1} \tan \beta-\frac{d}{\cos \beta}
$$

By rearranging Eq. (2.15) into an inequality constraint for both joints, the inequality constraints on the first edge of the link for the proximal and distal joint 


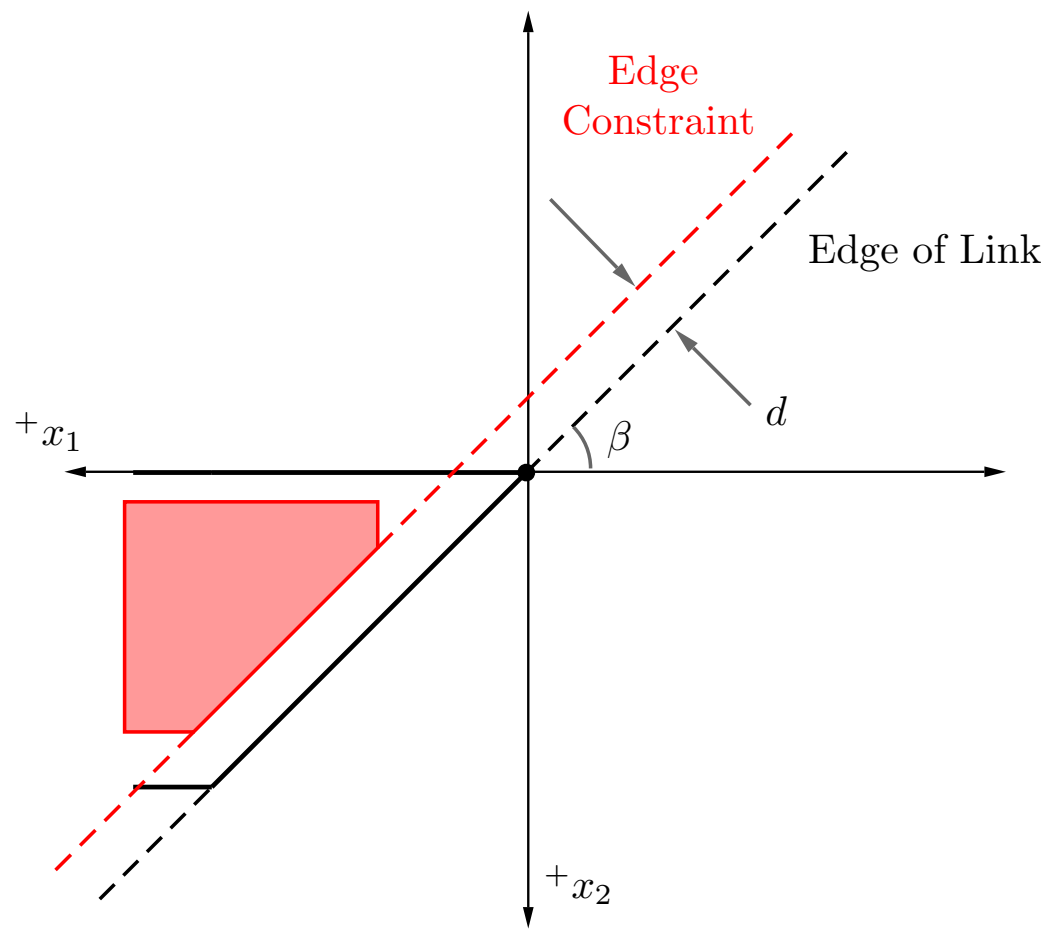

Figure 2.8: Coordinate system mapped to distal joint for the proximal link edge constraint.

problems are presented in Eqs. (2.16) and (2.17) respectively.

$$
\begin{gathered}
x_{1} \sin \beta_{b}-x_{2} \cos \beta_{b} \geq d_{b}-w \cos \beta_{b} \\
x_{1} \sin \beta_{p_{2}}-x_{2} \cos \beta_{p_{2}} \geq d_{p_{2}}
\end{gathered}
$$

where $\beta_{i}$ represents the angle of the edge with respect to the horizontal, $d_{i}$ represents the magnitude of the edge constraint distance, $w$ represents a dimension specific to the gripper base (see Fig. 2.7), and subscripts $b$ and $p_{2}$ and represent the gripper base and the second proximal link edge respectively.

As for the equality constraints on the angled edge of the second link, the constraint equations are formulated using the same technique used for the previous inequality constraints. As such, the edge equality constraints for the proximal and distal joints are given in Eqs. (2.18) and (2.19) respectively.

$$
x_{3} \sin \beta_{p_{1}}-x_{4} \cos \beta_{p_{1}}=d_{p_{1}}
$$




$$
x_{3} \sin \beta_{d}-x_{4} \cos \beta_{d}=d_{d}
$$

where the subscript $p_{1}$ represents the first angled edge on the proximal link, and $d$ represents the distal link. The reason that the constraints on the tendon termination location are given as an equality constraint are a result of physical properties of the obtained hardware. As it will be further described in Section 3.2.1, there is only one way that the spherical ferrule on the cable could be mounted to the link with the limited volume available on the links.

Finally, there exists a non-linear constraint on the magnitude of the moment arm, $r_{t}(\boldsymbol{x}, \theta)$, such that it is bound between zero and a user specified maximum $r_{\max }$. This is expressed by the nonlinear constraint in Eq. (2.20), where the magnitude of the moment arm was defined previously as per Eq. (2.10).

$$
0 \leq r_{t}(\boldsymbol{x}, \theta) \leq r_{\max }
$$

The constraints defined thus far have represented the physical bounds on the feasible region, and the bounds on the actual magnitude of the moment arm $r_{t}(\boldsymbol{x}, \theta)$ itself. These are hard constraints since they must be satisfied at the optimal solution. However, there also exists a soft constraint for this optimization problem.

\section{Possible Optimal Solutions and Soft Constraint Satisfaction}

Let us first consider the fictitious optimal solution for an arbitrary joint angle on the distal joint, as shown by Fig. 2.9.

Given an optimal solution of $\mathbf{x}$, it can be seen the the maximum moment arm can be achieved for an infinite number of solutions as long as the points lie on the line connecting $\left(x_{1}, x_{2}\right)$ and $\left(x_{3}, x_{4}\right)$ (hereinafter referred to as the optimal-line), and are within the feasible region. This implies that the solver may return a different optimal solution based on the starting point that was selected.

To correct this issue, a soft constraint on the optimization problem was included. Linguistically, the constraint is defined such that for a given optimal solution, the exposed tendon length $L_{e}$ must be minimized while $\mathbf{x}$ remains within the feasible region and lies upon the optimal-line. This constraint will not only allow the solver 


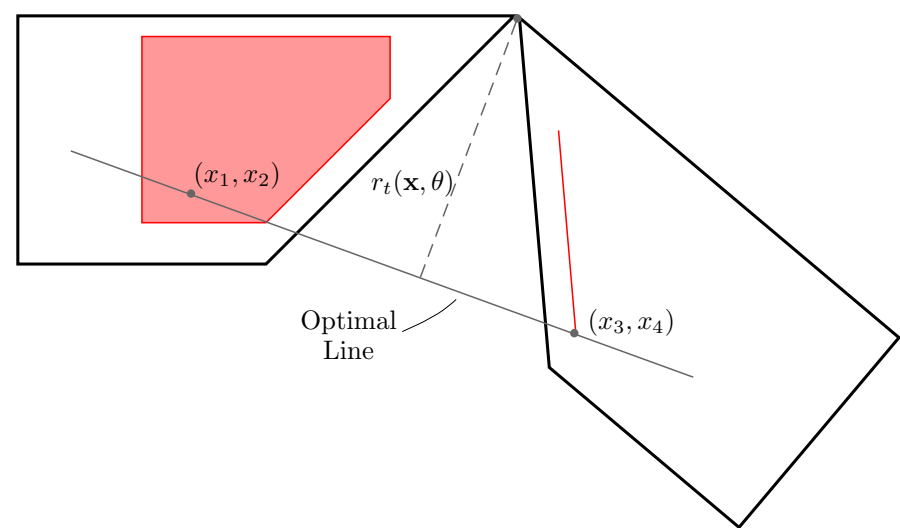

Figure 2.9: Demonstration of infinite optimal solutions on the distal joint.

to converge to the same solution for a given joint angle, but also reduce the size of the CSTM at the joint; making the joint more compact.

Several options were considered to implement this soft constraint in the optimization routine: 1) run a separate optimization routine with a different objective function to minimize $L_{e}$ for a given optimal-line, 2) select a software suite that could solve non-linear soft-constrained optimization problems, and 3) use a conditional statement to analytically determine the intersection of the feasible region bounds with the optimal-line. Of these options, option 3) was selected since an analytical expression is more accurate than the other options.

To implement option 3), the geometry of the feasible region and optimal-line for a given solution must first be defined. By mapping the feasible region and the optimal line to the coordinate system used in Fig. 2.8, the minimum exposed tendon length can be found by solving a system of linear equations to determine the desired point $\left(x_{1_{\text {des }}}, x_{2_{\text {des }}}\right)$, as per Fig. 2.10, while still ensuring that the new solution is within the feasible region.

This is accomplished by executing Algorithm 1, where the first step of the algorithm is to shift the optimal point $\left(x_{1_{\text {opt }}}, x_{2_{\text {opt }}}\right)$ along the optimum line until it intersects the edge constraint at $\left(x_{1_{t e m p}}, x_{2_{\text {temp }}}\right)$. Then, a series of conditional statements are executed to shift the point $\left(x_{1_{t e m p}}, x_{2_{\text {temp }}}\right)$ back along the optimal line if the hard constraints are violated. The definitions used in this algorithm are presented by the following expressions. 


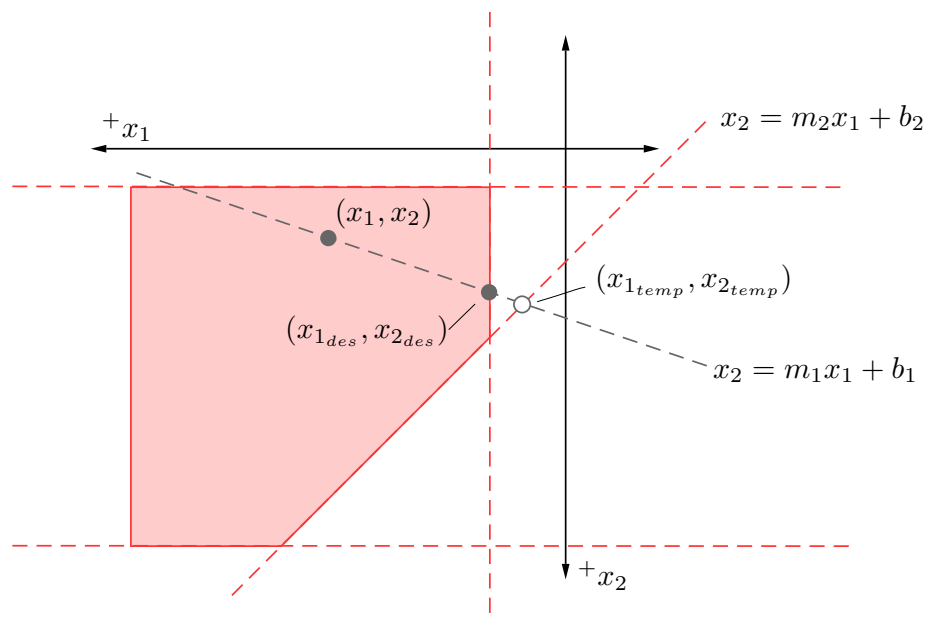

Figure 2.10: Optimal line and constraints mapped to the $x_{1}-x_{2}$ coordinate frame.

$$
\begin{gathered}
m_{1}=\tan \left(\beta_{b}\right) \\
b_{1}=-\frac{d_{b}}{\cos \left(\beta_{b}\right)}+w \\
m_{1}=\tan \left(\beta_{p_{2}}\right) \\
b_{1}=-\frac{d_{p_{2}}}{\cos \left(\beta_{p_{2}}\right)} \\
m_{2}=\tan \left(\alpha_{1}-\phi\right) \\
b_{2}=x_{2_{o p t}}-x_{1_{o p t}} \tan \left(\alpha_{1}-\phi\right)
\end{gathered}
$$

where the edge constraint line equation parameters are defined by Eqs. (2.21) and (2.22) for the proximal joint, and Eqs. (2.23) and (2.24) for the distal joint for the 
general form of $x_{2}=m x_{1}+b$. Equations (2.25) and (2.26) are the line parameters for the optimal line.

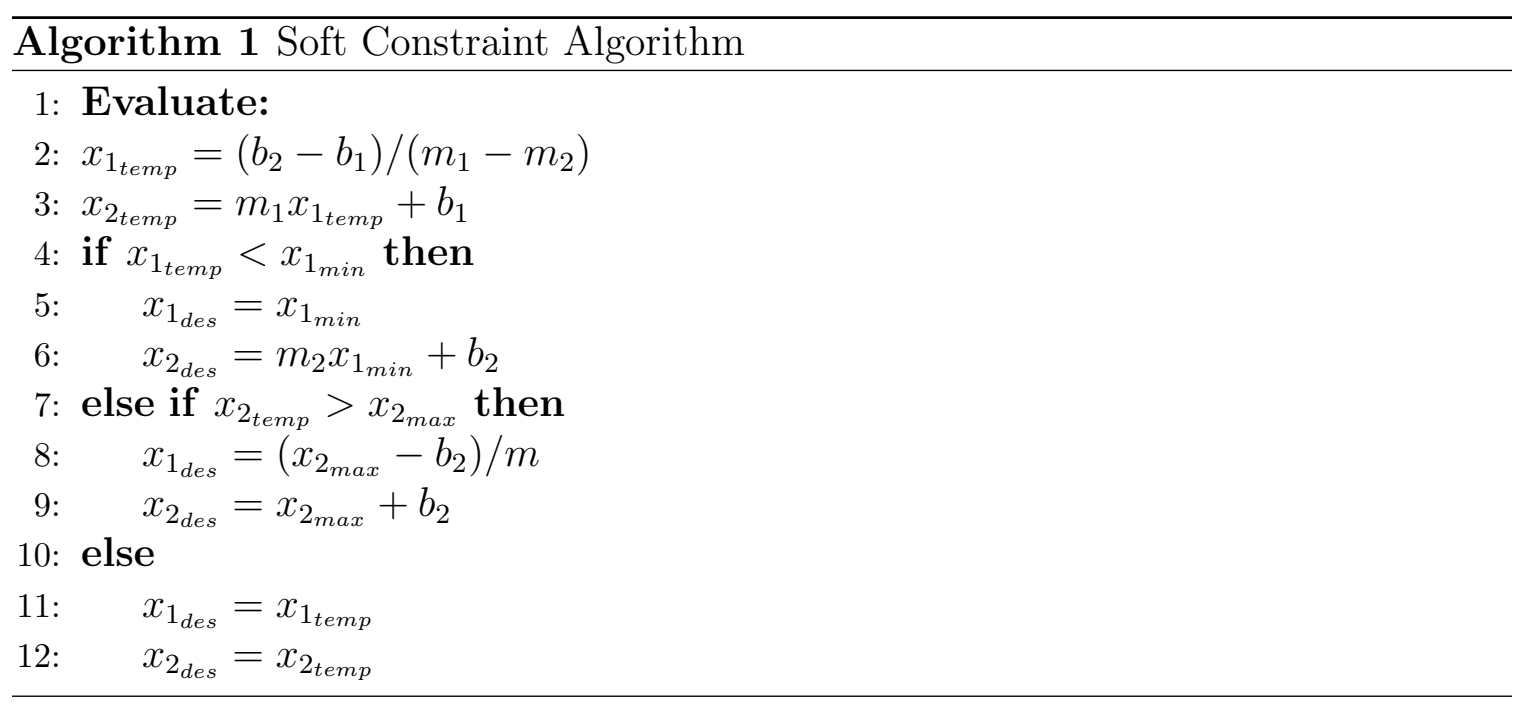

Algorithm 1 is to be executed following the optimization routines for the proximal and distal links. The details on the optimization algorithm selected, along with the software used to execute it are presented in the following section.

\section{Optimization Software}

As it was mentioned previously, the optimization problem must be solved for several cases since there are multiple joints and grip configurations, as described by Fig. 2.2. These are broken into the following cases: Case P1 is the proximal joint under GC-B and GC-C $\left(\theta_{p}=-45\right.$ degrees), Case P2 is the proximal joint under GC-A $\left(\theta_{p}=-90\right.$ degrees), Case D1 is the distal joint under GC-C $\left(\theta_{d}=0\right.$ degrees $)$, and Case D2 is the distal joint under GC-A and GC-B ( $\theta_{p}=90$ degrees).

Due to the presence of the non-linear boundary constraints on the cost function in Eq. (2.20), the problem requires a constrained, non-linear optimization tool in order to find the optimal solutions. Therefore, the MATLAB function fmincon (version 2017b) was selected due to its availability, computational fidelity, and ease of use [49]. This function allows the user to customize several settings, including: the solver algorithm, tolerances, maximum iterations, and step sizes, among many others.

The solver algorithms available within fmincon include interior point, sequential 
quadratic programming (SQP), trust-region-reflective, and active-set. The algorithm selected for this thesis was the SQP method since it offered more accurate solutions than interior point for this problem. Additionally, SQP allows all types of constraints simultaneously (unlike test-region-reflective), and it can recover from $\mathrm{NaN}$ or inf numeric errors (unlike active-set) [49]. Furthermore, the SQP algorithm has the Karush-Kuhn-Tucker (KKT) first order optimality check built into it [50].

With the solver algorithm selected, the tolerances on both the relative first-order optimality check and the maximum constraint violation were modified to be $10^{-9}$ while all other numerical settings in fmincon were left as the default values. For details on how the optimization problem was implemented in MATLAB, please refer to Appendix B.1.

\section{Optimal Solutions and Analysis}

Following the execution of the optimization routine, there were two optimal solutions per joint based on the angles of interest derived from the different grip configurations (see Table 2.1). Given that these solutions reflect the physical placement for the CSTM components, only one solution can be implemented at each joint.

Table 2.1: Optimal Solutions for Each Case.

\begin{tabular}{|c|c|c|c|c|}
\cline { 2 - 5 } \multicolumn{1}{c|}{} & $x_{1}$ & $x_{2}$ & $x_{3}$ & $x_{4}$ \\
\hline \hline Case P1 & $4.74 \mathrm{~mm}$ & $15.00 \mathrm{~mm}$ & $4.66 \mathrm{~mm}$ & $10.00 \mathrm{~mm}$ \\
\hline Case P2 & $2.50 \mathrm{~mm}$ & $12.76 \mathrm{~mm}$ & $4.66 \mathrm{~mm}$ & $10.00 \mathrm{~mm}$ \\
\hline Case D1 & $14.00 \mathrm{~mm}$ & $9.00 \mathrm{~mm}$ & $11.24 \mathrm{~mm}$ & $10.30 \mathrm{~mm}$ \\
\hline Case D2 & $12.75 \mathrm{~mm}$ & $9.00 \mathrm{~mm}$ & $11.24 \mathrm{~mm}$ & $10.30 \mathrm{~mm}$ \\
\hline
\end{tabular}

Since each solution will affect the performance of the joint differently throughout all operational angles, an analysis of the overall performance of each solution must be considered to determine the better solution. To do so, the moment arm at every integer joint angle was found by applying Eq. (2.10) for each solution highlighted in Table 2.1. For the purpose of comparison, the maximum moment arm at all joint angles for each joint was also determined using the optimization routine highlighted 
in Section 2.2.2. These performance curves are found in Fig. 2.11 and Fig. 2.12 for the proximal and distal joints respectively, where the vertical axis corresponds to the magnitude of the torque arm and the horizontal axis represents the joint angle. Additionally, the efficiency of each case can be seen as per Fig. 2.13 and Fig. 2.14, where the vertical axis represents the percentage of torque transmission relative to the the optimal case for a given joint angle in the horizontal axis. These figures are plotted for each joint as a way to quantitatively determine the more suitable solution.

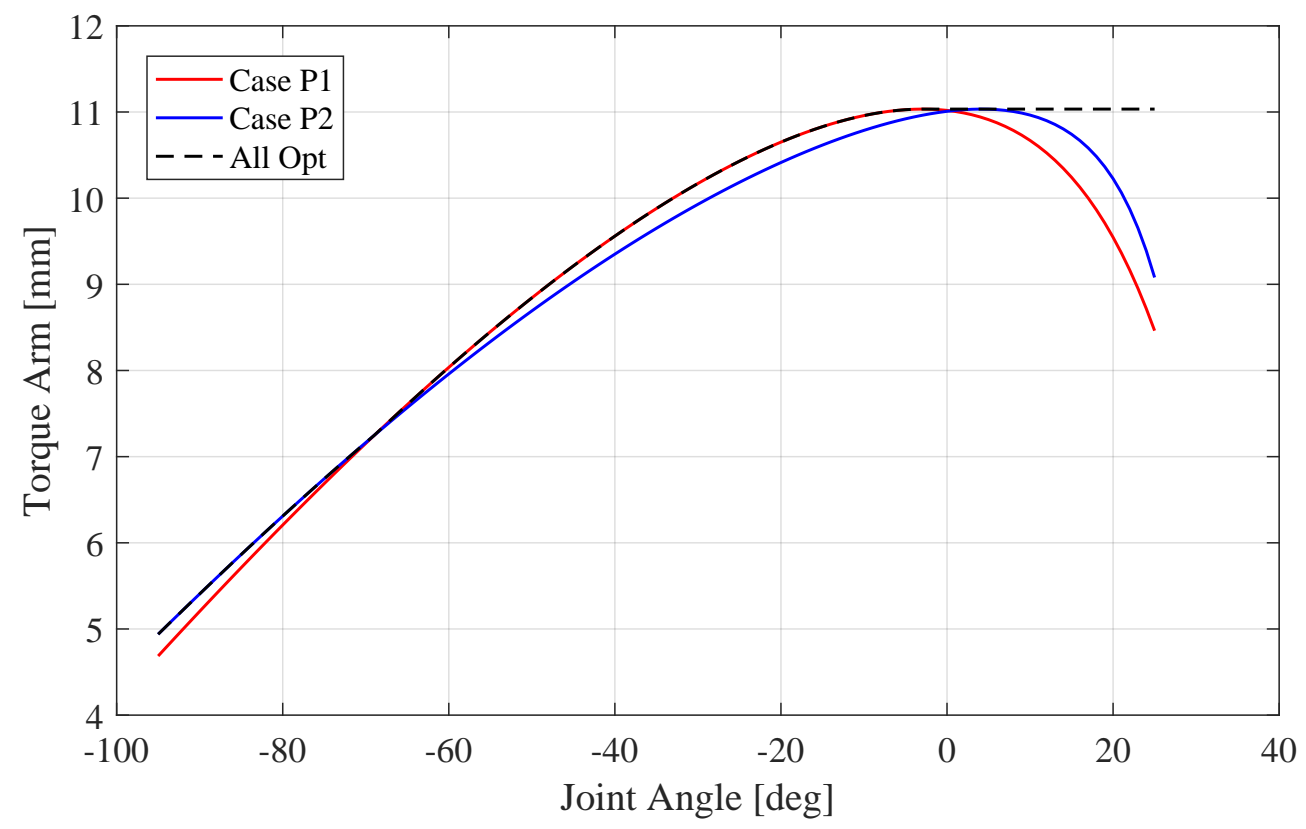

Figure 2.11: Moment arm performance comparison of Case P1 and Case P2 compared against the optimal case as a function of joint angle.

Let us first consider the performance of each proximal joint case (Case P1, and Case P2). In general, Case P1 matches the optimal curve for more joint angles than Case P2 implying that it has optimal performance at more than just the desired -45 degrees. However, when looking at the joint angles for all possible grip configurations $\left(\theta_{p}=-45\right.$ degrees, and $\theta_{p}=-90$ degrees $)$ in Fig. 2.13, the percent efficiency shows that Case P2 will have a better collective performance over Case P1 (see Table 2.2). For this reason, the solution for Case P2 will be implemented into the gripper design for the proximal joint.

The distal joint cases (Case D1, and Case D2) have a similar trend to the proximal 


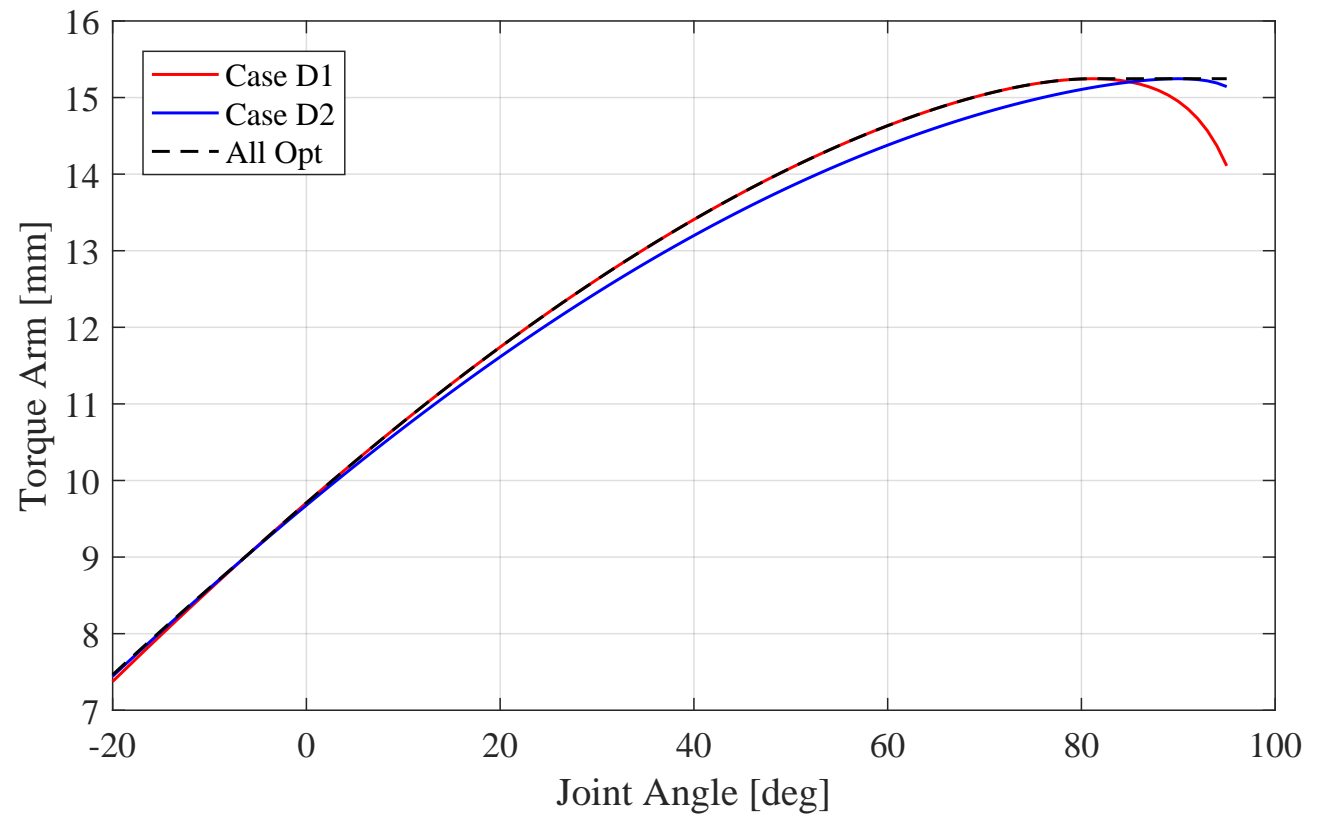

Figure 2.12: Moment arm performance comparison of Case D1 and Case D2 compared against the optimal case as a function of joint angle.

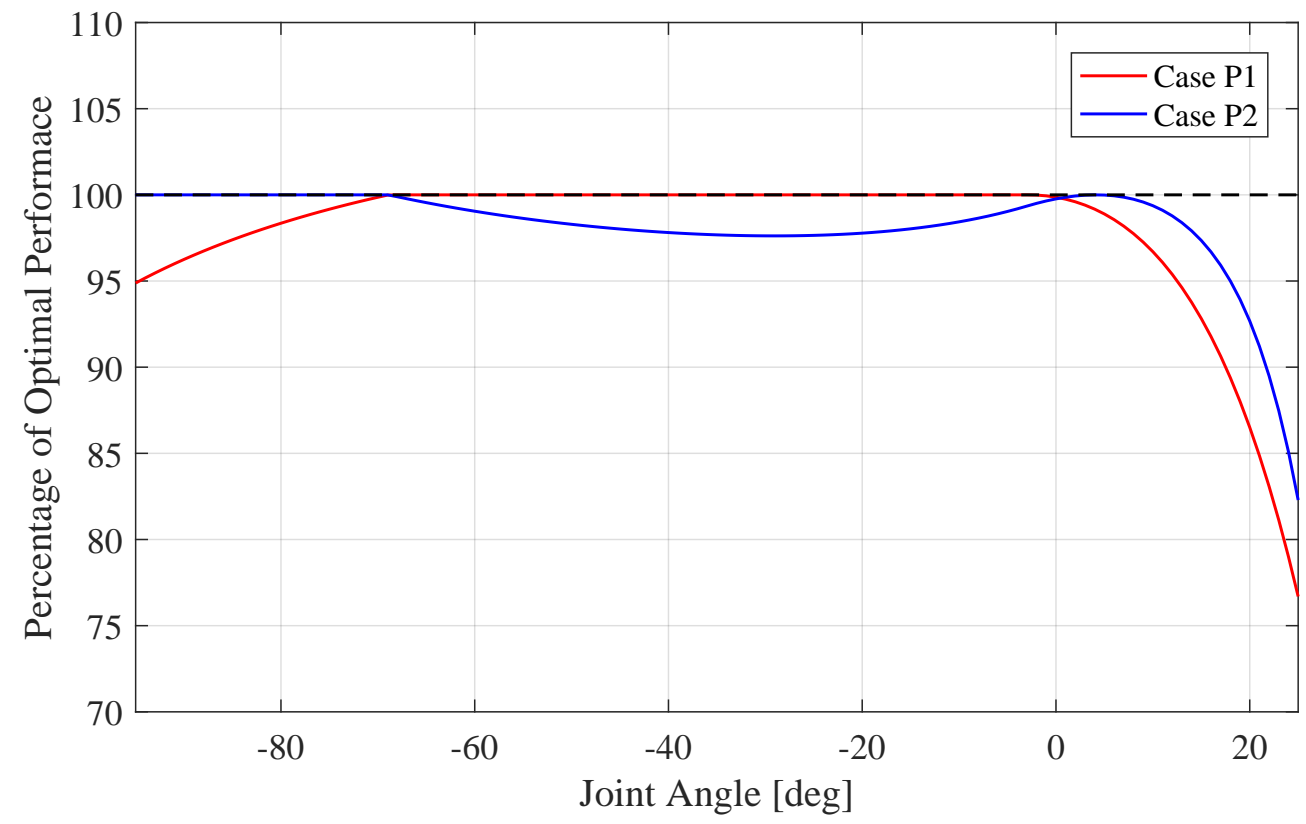

Figure 2.13: Performance efficiency relative to optimal curve for Case P1 and Case P2. 


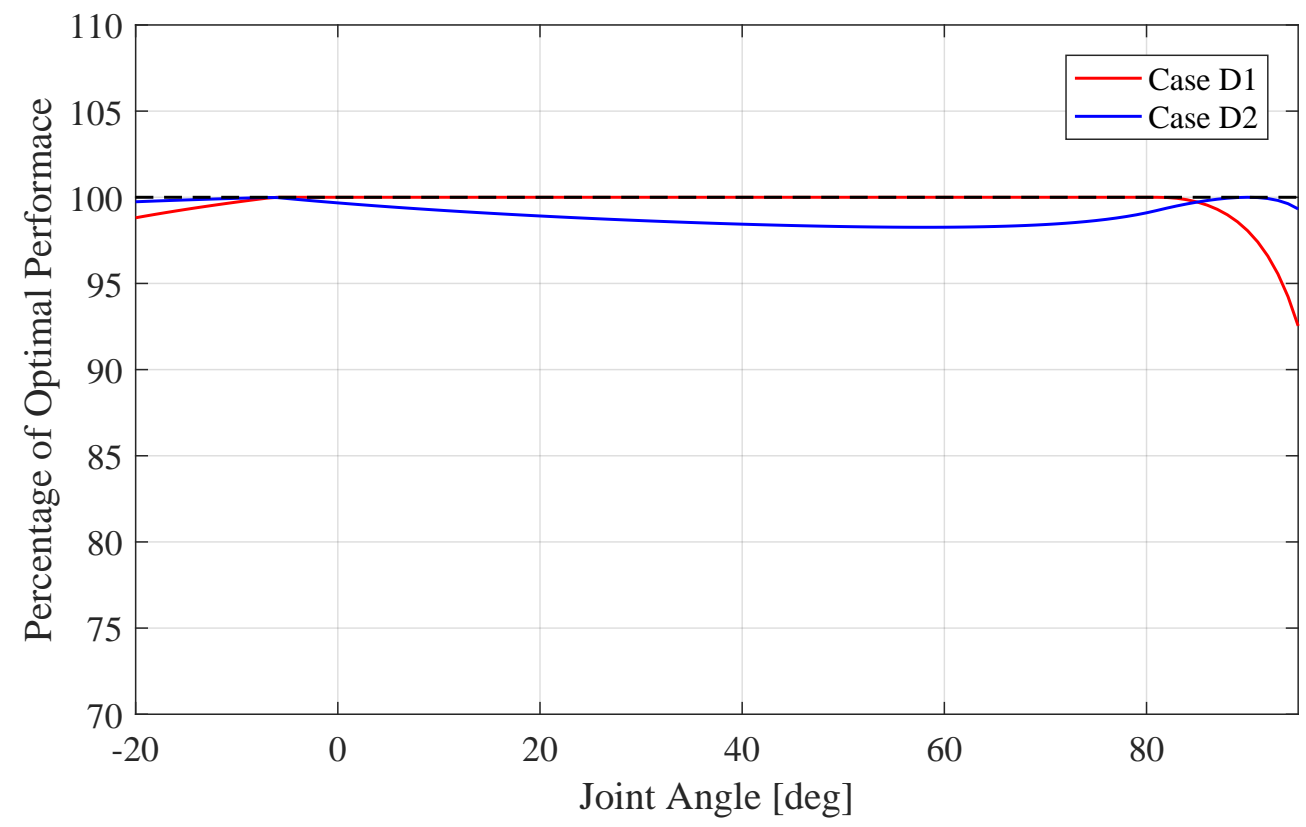

Figure 2.14: Performance efficiency relative to optimal curve for Case D1 and Case D2.

Table 2.2: Performance Efficiency for Proximal Joint.

\begin{tabular}{|c|c|c|}
\cline { 2 - 3 } \multicolumn{1}{c|}{} & @-45 deg & @-90 deg \\
\hline \hline Case P1 & $100.0 \%$ & $96.26 \%$ \\
\hline Case P2 & $98.02 \%$ & $100.0 \%$ \\
\hline
\end{tabular}

joint. When considering the joint angles of interest based on the desired grip configurations $\left(\theta_{d}=0\right.$ degrees, and $\theta_{d}=90$ degrees), Case D2 demonstrates a slightly better performance over Case D1 (see Table 2.3). Therefore, Case D2 will be implemented into the design of the distal joint.

\subsection{Actuation Design}

With the joint configuration selected, the actuation subsystem of the gripper can now be designed. This subsystem is defined to contain all of the components which affect the motion of the gripper; among which include the actuating motors, the CSTM used to transmit the load to the links, and the restitution springs in the 
Table 2.3: Performance Efficiency for Distal Joint.

\begin{tabular}{|c|c|c|}
\cline { 2 - 3 } \multicolumn{1}{c|}{} & @-45 deg & @-90 deg \\
\hline \hline Case D1 & $100.0 \%$ & $98.03 \%$ \\
\hline Case D2 & $99.67 \%$ & $100.0 \%$ \\
\hline
\end{tabular}

joints used to return the gripper to its neutral position. It can be said that the primary objective of the actuation subsystem is to maintain a rigid capture for all grip configurations outlined in Fig. 2.2 for a variety of manoeuvres. To simplify the sizing analysis, the actuators will be sized to withstand the worst-case manoeuvre for each grip configuration. Therefore, this section will cover the manoeuvre requirements for each grip configuration in Section 2.3.1, the CSTM design in Section 2.3.2, and the sizing of the actuator and restitution springs in Section 2.3.3.

\subsubsection{Grip Requirements}

To determine the worst-case manoeuvre for each grip configuration, the operational limits of the target manipulation manoeuvres must first be defined. It was stipulated that the target platform shall not be manipulated at an acceleration greater than $a_{p}=0.1 \mathrm{~m} / \mathrm{s}^{2}$ in any direction, nor shall it exceed an angular acceleration greater than $\alpha_{p}=0.25 \mathrm{rad} / s^{2}$ based on the specifications of the SRCL. To translate this requirement into a maximum required joint torque for each grip configuration, the interactions between platform and gripper must be determined. Since the moment arm for the links will vary as a function of angle as per Eq. (2.10), it would be more useful to determine the required cable tension at the joint to accomplish the manoeuvre. Thus, the following sections will outline the relationship between the worst-case manoeuvre and the required cable tension.

\section{Grip Configuration A}

Under normal operational conditions, the primary mechanism responsible for maintaining a rigid capture in GC-A is the adhesion of the contact interface on the target rather than the closing strength of the gripper. However, once captured, the links 
must resist any inertial loading of the target during manipulation. Let us examine the specific case where the target is already captured and the gripper must induce the maximum angular and linear acceleration on the target for a detumbling manoeuvre, as per Fig. 2.15.

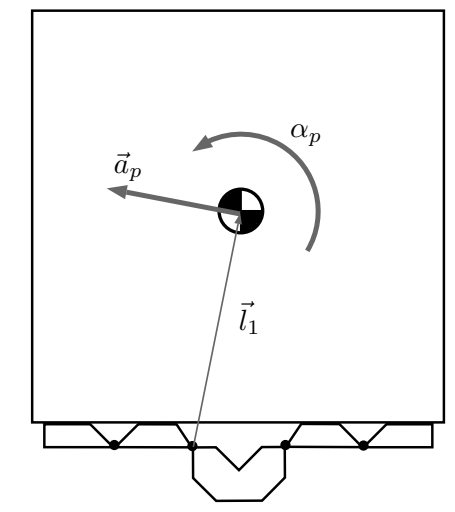

Figure 2.15: Detumbling manoeuvre of target under GC-A.

Since the failure of the gripper is being considered (not the failure of the adhesive), it is assumed that the adhesion between the target and contact interface is a rigid connection. This implies that all of the inertial back-torque due to the platform is loaded entirely on the proximal joint since there will be no relative motion between the proximal and distal links (i.e. no torque at the distal joint). Furthermore, it can be assumed that the manoeuvre is acting exclusively on one side of the gripper to synthesize a worst case loading scenario. These assumptions, though pessimistic, will ensure that the maximum required joint torque is determined conservatively, thereby guaranteeing its performance under normal operating conditions. Figure 2.16 represents the free-body diagram $(\mathrm{FBD})$ of this simplified case, where the body shown is the lumped mass of the gripper links and the target platform.

In Fig. 2.16, $T_{c}$ is the torque generated by the cable, $T_{s}$ is the torque generated by the spring, and $T_{I}$ is the inertial back torque generated by the angular and linear acceleration of the target platform ( $\alpha_{p}$ and $\vec{a}_{p}$ respectively). For later convenience, $I_{T}$ is defined generally as the the combined mass moment of inertia of the platform and the link assembly about the proximal joint. Note that for each capture case, there is a unique $I_{T}$ associated to it; that is to say, $I_{T_{A}}$ corresponds to GC-A, $I_{T_{B}}$ corresponds 


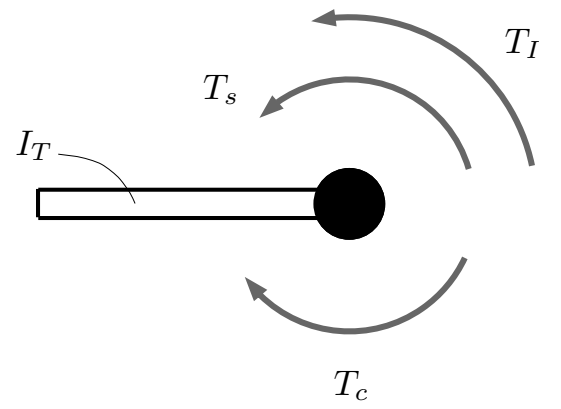

Figure 2.16: FBD of detumbling manoeuvre of target under GC-A.

to GC-B, an $I_{T_{C}}$ corresponds to GC-C. For the system to be in equilibrium, the sum of torques about the proximal joint must be zero.

$$
\sum T=0=T_{c}+T_{s}+T_{I}
$$

By definition, the torque generated by the cable is simply the product of the torque arm $r_{t}(\mathbf{x}, \theta)$ (see Eq. (2.10)) and the magnitude of the cable tension at the output end $F_{o_{A}}$, as per Eq. (2.28) below. Note that for the proximal link, $\mathbf{x}=\mathbf{x}_{p}$ remains constant as it is the selected design configuration for the proximal joint (refer to Case P2 in Table 2.1).

$$
T_{c}=F_{o_{A}} r_{t}\left(\mathbf{x}_{p}, \theta\right)
$$

The back torque due to the spring is defined by Eq. (2.29), where $k_{s p}$ is the torsional spring constant of the proximal joint spring, and $\theta_{0}$ is the initial angular displacement of the spring.

$$
T_{s}=k_{s p}\left(\theta-\theta_{0}\right)
$$

The inertial torque $T_{I}$ is simply the sum of the angular and linear accelerations as per Newton's second law.

$$
T_{I}=I_{T_{A}} \alpha_{p}+m_{p}\left|\vec{a}_{p} \times \vec{l}_{1}\right|
$$

where $m_{p}$ is the mass of the target platform. To find $I_{T_{A}}$, parallel axis theorem has 
been applied to combine the mass moment of inertia of the link assembly about the proximal joint $I_{l}$, with the mass moment of inertia of the platform about its center of mass $I_{p}$ at a distance of $l_{1}$ from the point of rotation.

$$
I_{T_{A}}=I_{l}+I_{p}+m_{p} l_{1}^{2}
$$

Since the direction of the linear acceleration in this manoeuvre is unknown, it is assumed that the platform is manipulated in a direction that is perpendicular to the

vector $\vec{l}_{1}$, such that $\left|\vec{a}_{p} \times \vec{l}_{1}\right|=a_{p} l_{1}$ to maximize the possible inertial back torque on the joint. Therefore, by combining Eq. (2.28) to (2.30) into Eq. (2.27), the required cable tension at the proximal joint for a detumbling manoeuvre under GC-A can be resolved as per Eq. (2.32).

$$
F_{o_{A}}=\frac{k_{s p}\left(\theta-\theta_{0}\right)+I_{T_{A}} \alpha_{p}+m_{p} a_{p} l_{1}}{r_{t}\left(\mathbf{x}_{p}, \theta\right)}
$$

\section{Grip Configuration B}

For the corner grip manoeuvre, GC-B, one or both of the following two conditions must be met in order to maintain retention of the target during a pull manoeuvre: 1) there must be an adhesion force between the target and the gripper contact interface, or 2) in the absence of adhesion, the coefficient of friction between the target and contact interface must be greater than 1 . Since these interactions are entirely material dependent and can be easily modified post-manufacture based on the link design (see Section 3.2 for details), they cannot be accurately incorporated into the sizing analysis.

Instead, a similar analysis to GC-A will be performed, it is assumed that the target has already been successfully captured and must be manipulated at angular and linear accelerations of $\alpha_{p}$ and $\vec{a}_{p}$ respectively in a push manoeuvre. This scenario is depicted in Fig. 2.17.

As such, a similar assumption will be made as in GC-A, where it is assumed that the target is in a rigid no-slip condition with the contact interface of the gripper. Furthermore, as with the analysis of GC-A, it is assumed that the target platform is loaded entirely on one side of the gripper. This means that the mass properties of 


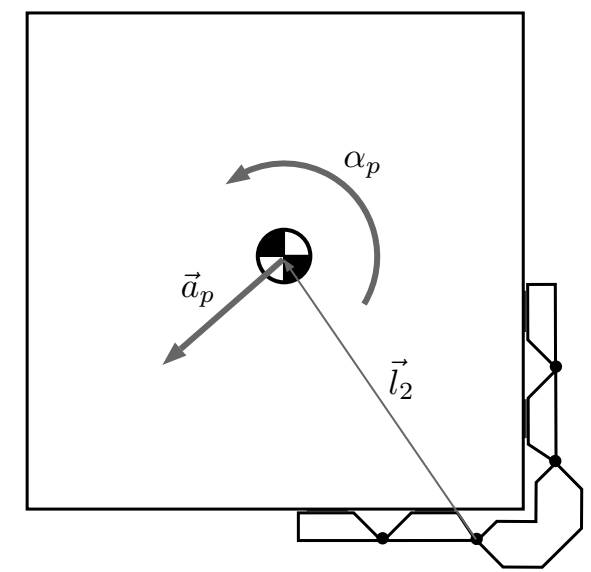

Figure 2.17: Push manoeuvre of the target in GC-B.

the target platform and link assembly can be combined in a similar way as with the FBD from Fig. 2.16.

Since this scenario is nearly identical to the analysis performed in Section 2.3.1, we can simply rewrite the definition of the total mass moment of inertia with respect to the new position vector $\overrightarrow{l_{2}}$ as per Eq. (2.33), and the total inertial back-torque on the link assembly as per Eq. (2.34).

$$
\begin{aligned}
& I_{T_{B}}=I_{l}+I_{p}+m_{p} l_{2}^{2} \\
& T_{I}=I_{T_{B}} \alpha_{p}+m_{p} a_{p} l_{2}
\end{aligned}
$$

Given that the cable torque $T_{c}$ and spring torque $T_{s}$ defined for GC-A are the same for GC-B, the torque balance will yield the Eq. (2.35) for the cable tension required to retain the GC-B grip during the worst case manoeuvre.

$$
F_{o_{B}}=\frac{k_{s p}\left(\theta-\theta_{0}\right)+I_{T_{B}} \alpha_{p}+m_{p} a_{p} l_{2}}{r_{t}\left(\mathbf{x}_{p}, \theta\right)}
$$

\section{Grip Configuration C}

Unlike the other grip configurations, GC-C is an enveloping grasp which does not require an adhesive contact pad to retain a rigid capture. Since the distal links are 
what envelop the target handle, retention would be lost if the target is manipulated such that the inertial loads will exceed the closing strength of the distal links. Thus, as a conservative approach, it will be assumed that the entirety of the inertial loads will be transmitted through a single distal link as per Fig. 2.18.

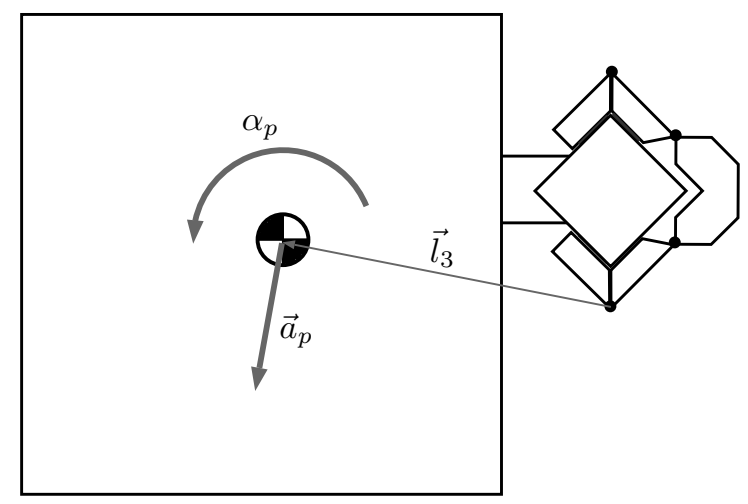

Figure 2.18: Pull manoeuvre of the target in GC-C.

Once again, it will also be assumed that the target handle is in a no slip condition with the contact interface at the moment of failure such that the inertial properties of the link assembly and target platform can be lumped as a single rigid body, as by the FBD in Fig. 2.16. The total mass moment of inertia of this lumped body is presented in Eq. (2.36).

$$
I_{T_{C}}=I_{l}+I_{p}+m_{p} l_{3}^{2}
$$

In the case of GC-C, the cable torque is a function of the distal link sheath placement $\mathbf{x}_{d}$ (Case D2 in Table 2.1), which is a constant design parameter.

$$
T_{c}=F_{o_{C}} r\left(\mathbf{x}_{p}, \theta\right)
$$

Similarly, the torque caused by the torsion spring is now a function of the stiffness $k_{s d}$, which is unique to the distal joints.

$$
T_{s}=k_{s d}\left(\theta-\theta_{0}\right)
$$

Finally, as with the other two manoeuvres, the combined inertial back-torque on the 
link is simply the sum of the angular and linear inertial torques of the target platform.

$$
T_{I}=\alpha_{p} I_{T_{C}}+m_{p} a_{p} l_{3}
$$

Thus, after performing the torque balance of Eqs. (2.37) to (2.39), the required cable tension for GC-C under the maximum expected accelerations is presented below.

$$
F_{o_{C}}=\frac{k_{s d}\left(\theta-\theta_{0}\right)+I_{T_{C}} \alpha_{p}+m_{p} a_{p} l_{3}}{r_{t}\left(\mathbf{x}_{d}, \theta\right)}
$$

\subsubsection{CSTM Design}

With the required output tensions defined for each worst-case grip scenario, it is now necessary to derive the load transmission dynamics of the CSTM to determine what input loads are required. As alluded to previously, CSTMs are non-linear hysteretic systems due to friction between the cable and sheath. There are several models which exist for characterizing the force transmission in CSTMs, however for the sake of simplicity, the model developed by Kaneko et al. [38] based on the Coulomb friction model will be used in this section. The accuracy of this model is quite suitable for a sizing analysis, as proven by Lin [37] and Palli [41].

First, consider an infinitesimally small tendon element with a length of $d x$ subtending an arc of $d \gamma$ radians, as per Fig. 2.19, where $F_{N}(x)$ is the normal force of the sheath acting against the tendon, $F_{F}(x)$ is the force of friction acting on the tendon element, and $F_{T}(x)$ is the tension applied at the input which varies by $d F_{T}(x)$ to the output.

By small angle theory, one can assume that the normal force can be approximated to be $F_{N}(x) \simeq F_{T}(x) d \gamma$, such that the maximum Coulomb friction acting on the element can be defined with the following relationship.

$$
F_{F}(x)=\mu_{s} F_{N}(x)=\mu_{s} F_{T}(x) d \gamma
$$

By recognizing that the arc element $d \gamma$ can be expressed in terms of the infinitesimal length $d x$, the friction can be expressed as a function of its length. Note that the curvature of the tendon element $R(x)$ is expressed as a function of $x$ for the sake 


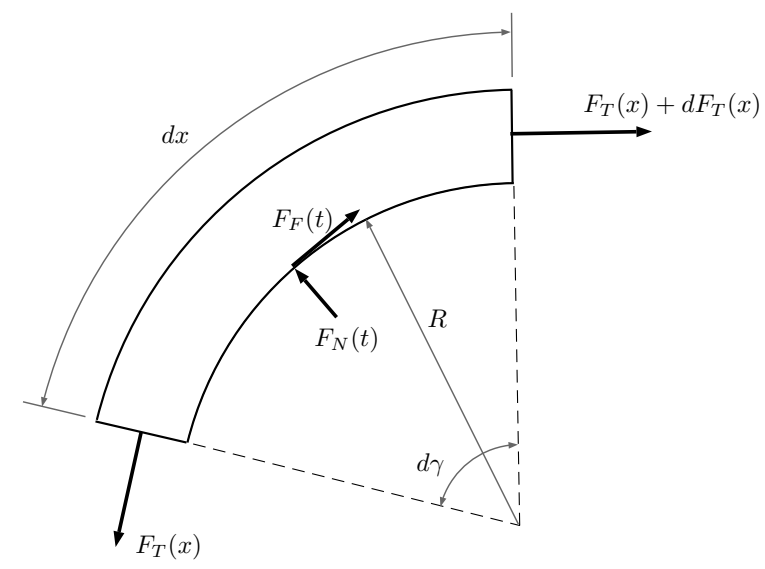

Figure 2.19: Infinitesimal tendon element within sheath.

of convenience later.

$$
F_{F}(x)=\frac{\mu_{s} F_{T}(x)}{R(x)} d x
$$

Since only the magnitude of the friction force is required for this sizing analysis, the direction of motion is not included in this form of the expression. By assuming that the angle between the input and output is infinitely small, the force balance on the tendon element simplifies as follows.

$$
\begin{gathered}
F_{T}(x)=F_{F}(x)+\left(F_{T}(x)+d F_{T}(x)\right) \\
d F_{T}(x)=-\frac{\mu_{s} F_{T}(x)}{R(x)} d x
\end{gathered}
$$

The analytical solution to Eq. (2.44) can be found by integrating both sides to give the tension of the cable at some distance $x$ along the length of the entire sheath $L$.

$$
F_{T}(x)=\left\{\begin{array}{lc}
F_{T_{i}} \exp \left(-\mu_{s} \int_{0}^{x} \frac{1}{R(x)} d x\right), & 0 \leq x \leq L_{1} \\
F_{T_{0}}, & L_{1} \leq x \leq L
\end{array}\right.
$$

Note that for a sheath length $L$, there is a point along the tendon at $x=L_{1}$ for $L_{1} \leq L$ where a commanded input tension $F_{T_{i}}$ does not affect the output tension $F_{T}(L)$. This can be seen by Fig. 2.20, where tension of the cable $F_{T}(x)$ is plotted as 
a function of distance along the CSTM $x$. The phenomenon of a given input being unable to command a reaction at the output is caused by the input tension being unable to overcome the total friction within the CSTM. One can find the critical point $x=L_{1}$ by solving $F_{T}(x)=F_{T_{0}}$, where $F_{T_{0}}$ is the pretension in the cable.

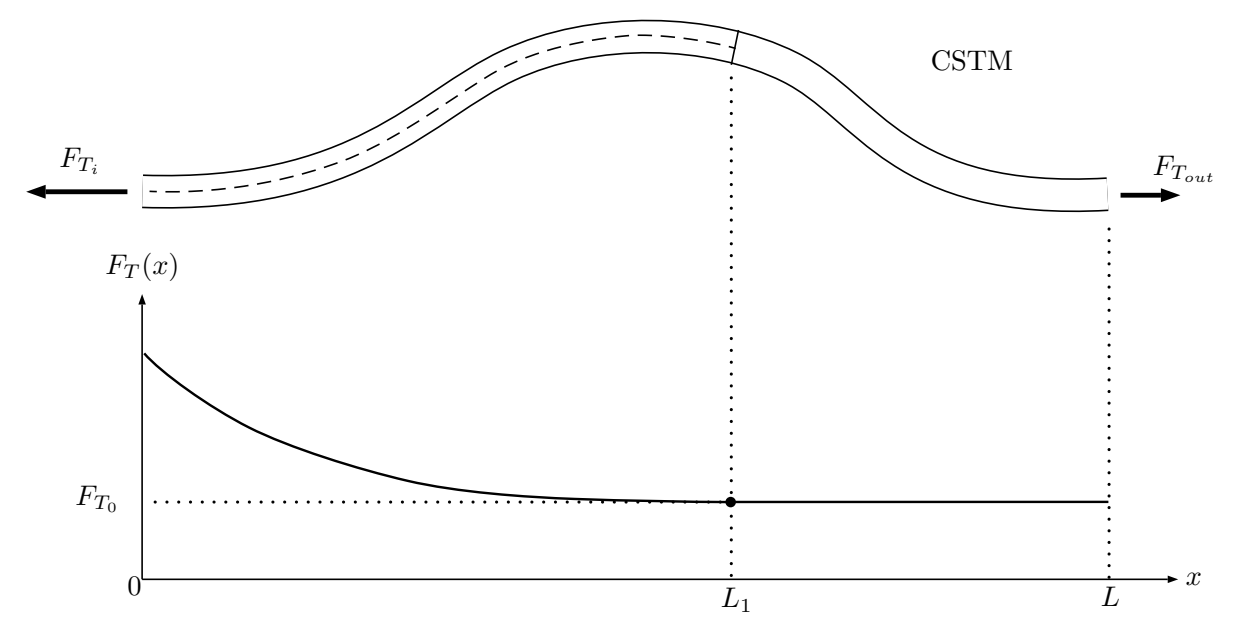

Figure 2.20: Critical point within CSTM.

Once the critical point is $L_{1}=L$, an input load will cause an nigh-instantaneous response to the output. Since we are interested in the maximum cable tension required at the input for the purposes of sizing the actuators, only the first part of Eq. (2.45) is necessary. By evaluating this expression over the whole length of the tendon, a continuous function for the ratio of output tension to input tension can be found, as per Eq. (2.46).

$$
\frac{F_{T_{\text {out }}}}{F_{T_{i}}}=\exp \left(-\mu_{s} \int_{0}^{L} \frac{1}{R(x)} d x\right)
$$

A consequence of this model is that one must know the curvature of the sheath as a function of position along the tendon. Fortunately, it can be shown that the integral of the CSTM curvature over its entire length is simply the total bend angle throughout the entire sheath $\Theta_{T}[37,38,41]$, which was further demonstrated experimentally by Do [44].

$$
\int_{0}^{L} \frac{1}{R(x)} d x=\Theta_{T}
$$


This simplifies the tension ratio in Eq. (2.46) to become solely a function of the static friction coefficient and the total curvature.

$$
\frac{F_{T_{\text {out }}}}{F_{T_{i}}}=\exp \left(-\mu_{s} \Theta_{T}\right)
$$

Since the maximum required actuator torque for a worst-case manoeuvre is desired, it will be assumed that the sheath will be routed along the arm and has a total bend angle equivalent to the sum of the arm and gripper joint limits (see Fig. 2.21). Note that this configuration does not reflect any of the proposed manoeuvres, as it is simply used as a pessimistic estimate for this analysis.

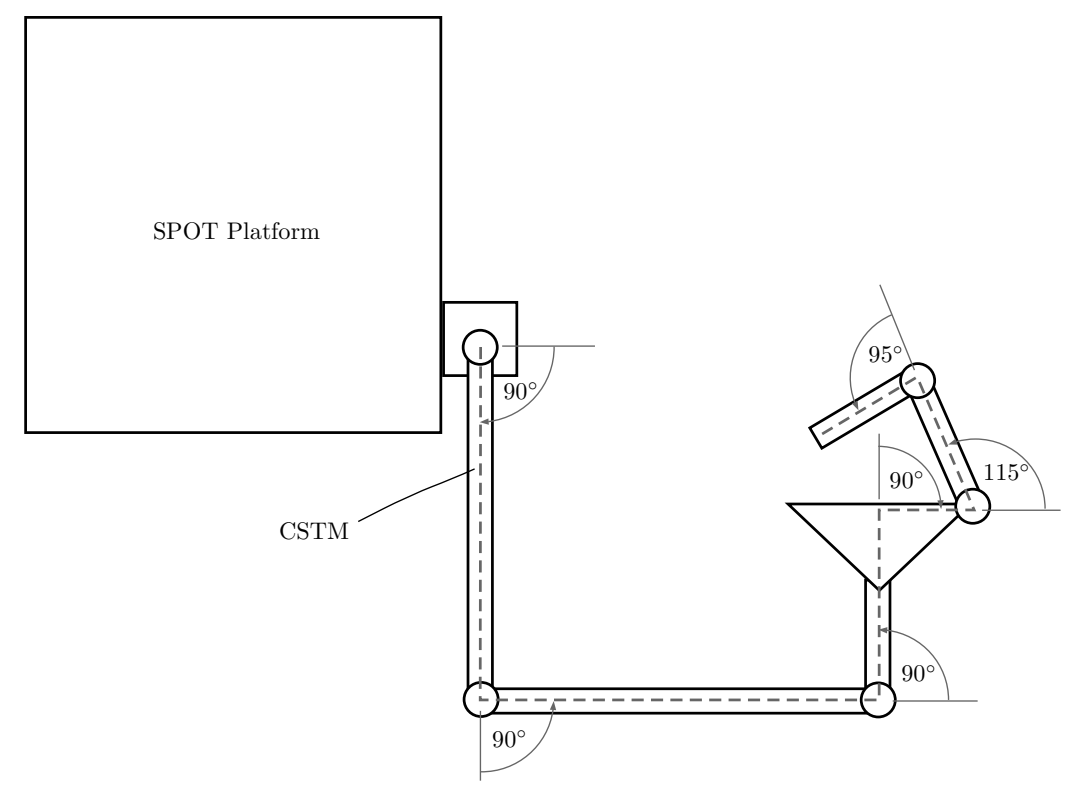

Figure 2.21: Worst-case sheath routing through arm and gripper.

From this schematic, the total bend angle of the CSTM is $\Theta_{T}=570 \mathrm{deg}(9.95$ radians). This value will be applied in the actuator sizing analysis as per Eq. (2.50) to (2.52). From Eq. (2.48), the only other parameter subject to design is the static friction coefficient between the sheath and cable. To minimize the transmission loss, it is common practice to use a stainless steel sheath with a polytetrafluoroethene (PTFE) coating [43], which has a coefficient of friction of 0.05-0.1 under varying conditions such as contact pressure and room temperature [51]. For the purposes of this analysis, coefficient of friction $\mu_{s}$ is assumed to be 0.1 as a conservative estimate. 


\subsubsection{Actuator and Spring Design}

Now that the required input tension can be found for each manoeuvre, the maximum required actuator torque can be determined. The actuation assembly for each link is assumed to be a winch style mechanism attached directly to the horn of a servomotor (see Fig. 2.3). The radius of the winch pulley $r_{p}$ is subject to design. For safety purposes, it is assumed that the scenarios outlined for GC-A, GC-B, and GC-C are performed at $80 \%$ of the actuator's stall torque. Thus, with $\eta=0.8$, the stall torque of the selected actuator should follow Eq. (2.49).

$$
T_{\max }=\frac{F_{T_{i}} r_{p}}{\eta}
$$

The required output tensions for GC-A, GC-B, and GC-C (Eqs. (2.32), (2.35), and (2.40), respectively) are then combined with the tension ratio (Eq. (2.48)) and the servomotor stall torque expression in Eq. (2.49). This will yield three expressions for the minimum stall torque required by the selected actuators to perform the expected tasks, given by Eq. (2.50) to (2.52) for grip configurations A to C respectively.

$$
\begin{aligned}
& T_{\text {max }_{A}}=\frac{\left(k_{s p}\left(\theta-\theta_{0_{p}}\right)+I_{T_{A}} \alpha_{p}+m_{p} a_{p} l_{1}\right) r_{p}}{\eta r_{t}\left(\mathbf{x}_{p}, \theta\right) \exp \left(-\mu_{s} \Theta_{T}\right)} \\
& T_{\text {max }_{B}}=\frac{\left(k_{s p}\left(\theta-\theta_{0_{p}}\right)+I_{T_{B}} \alpha_{p}+m_{p} a_{p} l_{2}\right) r_{p}}{\eta r_{t}\left(\mathbf{x}_{p}, \theta\right) \exp \left(-\mu_{s} \Theta_{T}\right)} \\
& T_{\text {max }_{C}}=\frac{\left(k_{s d}\left(\theta-\theta_{0_{d}}\right)+I_{T_{C}} \alpha_{p}+m_{p} a_{p} l_{3}\right) r_{p}}{\eta r_{t}\left(\mathbf{x}_{d}, \theta\right) \exp \left(-\mu_{s} \Theta_{T}\right)}
\end{aligned}
$$

where the values of $\theta$ are the joint angles for each corresponding grip configuration. Before expressions can be evaluated, there are several parameters that are subject to design: the torsion spring stiffnesses $k_{s p}$ and $k_{s d}$, the pretension angle of these springs $\theta_{0_{p}}$ and $\theta_{0_{d}}$, and the radius of the winch pulley $r_{p}$.

To determine these parameters, a MATLAB script was developed to evaluate Eqs. (2.50) to (2.52) based on selected hardware from commercial-off-the-shelf (COTS) products (see Appendix B.2). Through an iterative process, the design results in Table 2.4 were obtained. 
Table 2.4: Selected design parameters of actuation system.

\begin{tabular}{|c|c|c|}
\hline Parameter & Value & Units \\
\hline \hline$r_{p}$ & 11.0 & $\mathrm{~mm}$ \\
\hline$k_{s p}$ & $42.2 \times 10^{-3}$ & $\mathrm{~N} \cdot \mathrm{m} / \mathrm{rad}$ \\
\hline$\theta_{0_{p}}$ & 60 & $\mathrm{deg}$ \\
\hline$k_{s d}$ & $35.7 \times 10^{-3}$ & $\mathrm{~N} \cdot \mathrm{m} / \mathrm{rad}$ \\
\hline$\theta_{0_{d}}$ & 60 & $\mathrm{deg}$ \\
\hline
\end{tabular}

Table 2.5: Maximum required actuator torques for each manoeuvre.

\begin{tabular}{|l|c|c|}
\cline { 2 - 3 } \multicolumn{1}{c|}{} & Value & Units \\
\hline \hline$T_{\text {max }_{A}}$ & 1.47 & $\mathrm{~N} \cdot \mathrm{m}$ \\
\hline$T_{\text {max }_{B}}$ & 1.23 & $\mathrm{~N} \cdot \mathrm{m}$ \\
\hline$T_{\text {max }_{C}}$ & 1.25 & $\mathrm{~N} \cdot \mathrm{m}$ \\
\hline
\end{tabular}

The springs selected were sourced from Lee Spring with part numbers LTML100N $03 \mathrm{M}$ (left-hand wound) and LTMR100N 03 M (right-hand wound) for the proximal joints, and LTL038D $20 \mathrm{M}$ and LTR038D $20 \mathrm{M}$ for the distal joints. With these components selected, the required actuator torques from Eq. (2.50) to (2.52) were evaluated to give the required actuator torques for each manoeuvre, as reported in Table 2.5.

Note that the manoeuvre which requires the most actuation torque is GC-A, with a value of $1.47 \mathrm{~N} \cdot \mathrm{m}$. Based on this value, the MX-28 Dynamixel actuators were selected, with a stall torque of $2.5 \mathrm{~N} \cdot \mathrm{m}$ on a $12 \mathrm{~V}$ supply. It is recognized that the actuators exceed the requirement by a substantial margin, however, the MX-28 servos were selected for several reasons. The primary reason is that three of the required four were already procured from a previous iteration of the robotic arm. This meant that the integration into the existing laboratory software would be a trivial task and that the overall cost of the gripper would be reduced. Furthermore, the internal encoders of the MX-28 actuators are of 12 bit precision, allowing for smooth position control of the input winch. 


\section{Chapter 3}

\section{Experimental Facilities}

As stated previously, one of the main objectives of this thesis is that the capture mechanism developed must be implemented on the SPOT platform at Carleton University's SRCL. As such, the design must be compliant with the capabilities of the existing facilities, while still meeting the performance objectives outlined in Chapter 2. This chapter will therefore give a brief outline of the SPOT platform in Section 3.1, the mechanical design and integration of the gripper in Section 3.2, the instrumentation and measurement system used to acquired state feedback in Section 3.3, and an overview on the driving software in Section 3.4.

\subsection{SPOT Overview}

The SRCL SPOT is the largest free-floating spacecraft robotics testing facility in Canada. There are two key components to the facility: a large gravity offset surface, and an autonomous planar free-floating spacecraft analogue (see Fig. 3.1). The plat-

forms operate by forcing compressed air through a series of air bearings to create a near frictionless interface on the gravity offset surface; allowing planar 3-DOF motion to emulate the free-floating nature of spacecraft. 


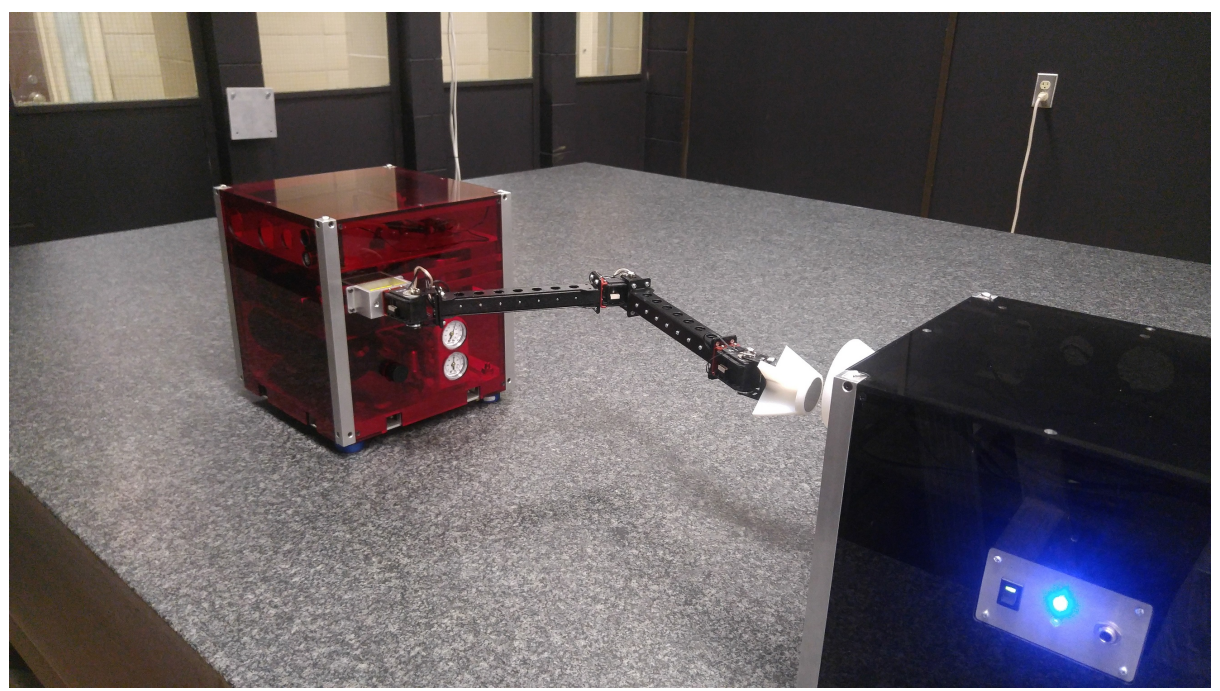

Figure 3.1: SPOT facilities: gravity offset surface and two free-floating platforms.

The on-board computer is a Raspberry Pi 3 (RP3) microcomputer running a Raspbian Jessie Linux distribution as its operating system. The RP3 executes commands which drive any actuated components, on-board instruments, and data logging of the ground truth system. For the purposes of the experimental work presented in this thesis, the platforms were assumed in a fixed-base configuration to isolate the gripper during its operation.

At the time of its conception in 2016, the SPOT platforms were developed as a collaborative effort by several graduate students and researchers. The structure and magnetic docking mechanism were developed by Justin Kernot (see Fig. 3.1), the avionics, propulsion, floatation, and ground truth systems were developed by Kirk Hovell [52], and the robotic arm and SPOT 2.0 software were developed by Alexander Crain [53]. For any further details that were not outlined in this section pertaining to the SRCL facilities, please refer to the resources provided [52-54].

\subsection{Gripper Assembly and Integration}

The key considerations for the mechanical design of the gripper are as follows: the gripper must interface with the wrist joint of the 3-link manipulator, the actuators driving the gripper must be mounted to the base of the platform, the gripper must be modular to allow for rapid installation/detachment from SPOT, and the contact 
interface of the gripper links must be easily interchangeable. Of the several designs that were considered, the selected configuration comprises two main components; the gripper, and the actuator bank.

\subsubsection{Gripper Assembly}

The gripper links were designed to reflect the optimal joint design outlined in Chapter 2 , such that the cable pin-off point and sheath mounting location comply with Case P2 and Case D2 in Table 2.1 for the proximal and distal joint respectively (refer to Appendix A for technical drawings). Figure 3.2 shows a rendering of the final design in its exploded view, and its assembled view. Note that the exploded view has been simplified due to the symmetry of the gripper; for more details on all the components, please refer to the full assembly drawing in Appendix A.1.
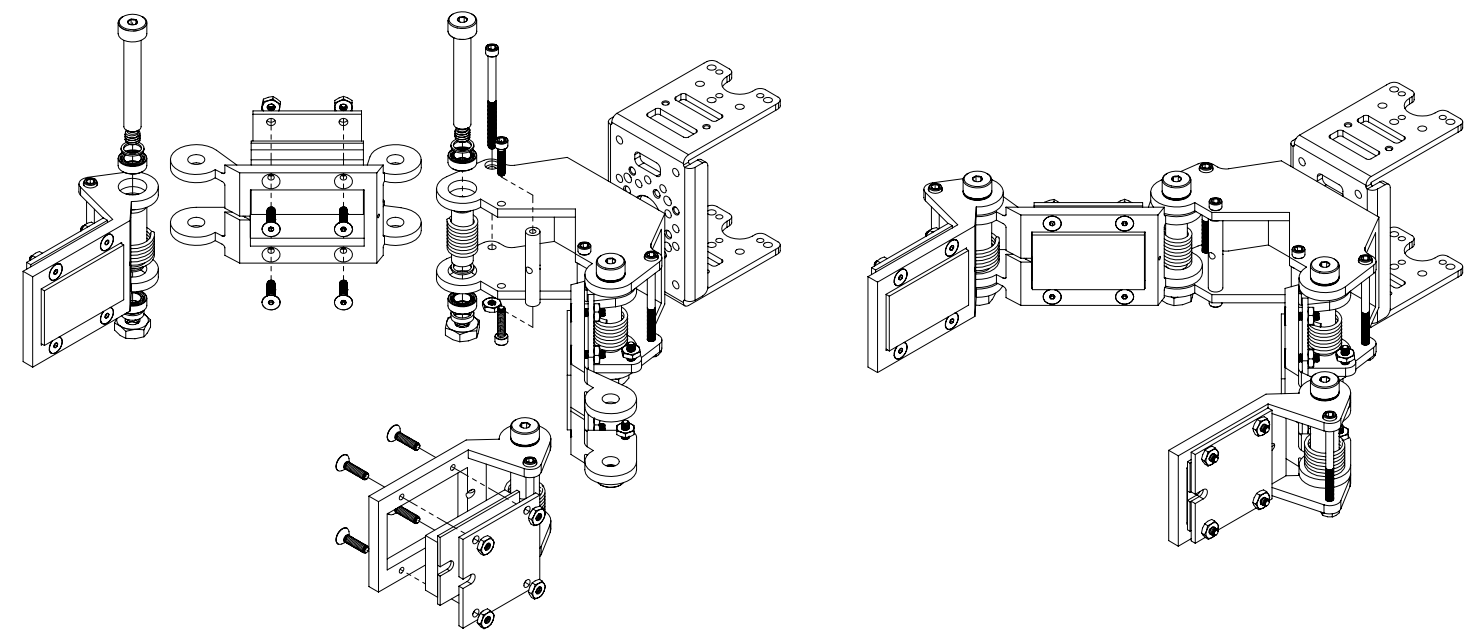

Figure 3.2: Gripper design. Exploded view (left) and assembled view (right).

In this design, the entire gripper is modular and does not require any permanent bonding methods to assemble, aside from press fitting the rotary bearings in their respective locations. This allows parts to be interchanged as necessary in the event of a component failure, or design revision. Rotational ball bearings were selected to reduce friction in the joint, ensuring smooth motion and effective force transmission. The contact pads are removable from each link by simply removing four hex nuts; allowing rapid interchangeability while avoiding the need for bonding materials to 
the links directly. Furthermore, the base of the gripper was designed to interface directly with the wrist bracket on the robotic arm with four screws, enabling a simple detachment method when interchanging EEs on the SPOT platform.

Once the author had procured the COTS components and manufactured the parts in-house with manual and CNC techniques, the gripper was assembled as seen by Fig. 3.3. The LEDs attached to the gripper are position tracking markers used by the PhaseSpace motion capture (ground truth) system, and will be further discussed in Section 3.3.

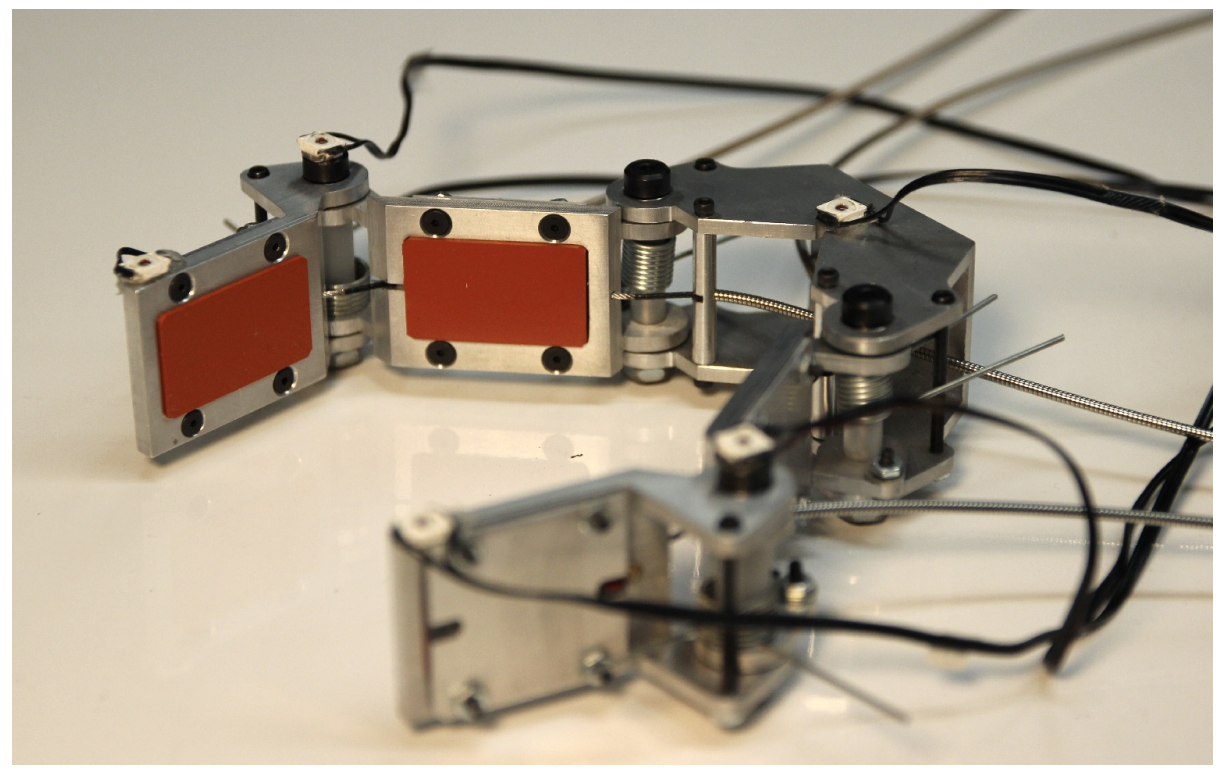

Figure 3.3: Fully assembled gripper.

\subsubsection{Actuation and Actuator Bank}

The CSTM selected in this assembly was sourced from Asahi Intecc Inc., where the cable is a $1.07 \mathrm{~mm}$ PTFE coated 7x7 braided SS304 wire rope with a spherical brass ferrule, and the sheath is a ID $1.2 \mathrm{~mm} \times$ OD $2.03 \mathrm{~mm}$ SS304 flat-wire coil. The tensile strength of the assembly was quoted at $300 \mathrm{~N}$ with a minimum bend radius of $8 \mathrm{~mm}$. On the gripper side, the sheaths are friction fitted into the terminal points within the links (Fig. 3.4) and the cables are attached to the pin-off point by resting the spherical ferrule into a socket where the wire rope can pass through (Fig. 3.5). Figure 3.4 also shows how the legs of the torsion spring are attached to the links to 
apply a restitution torque to open the gripper.
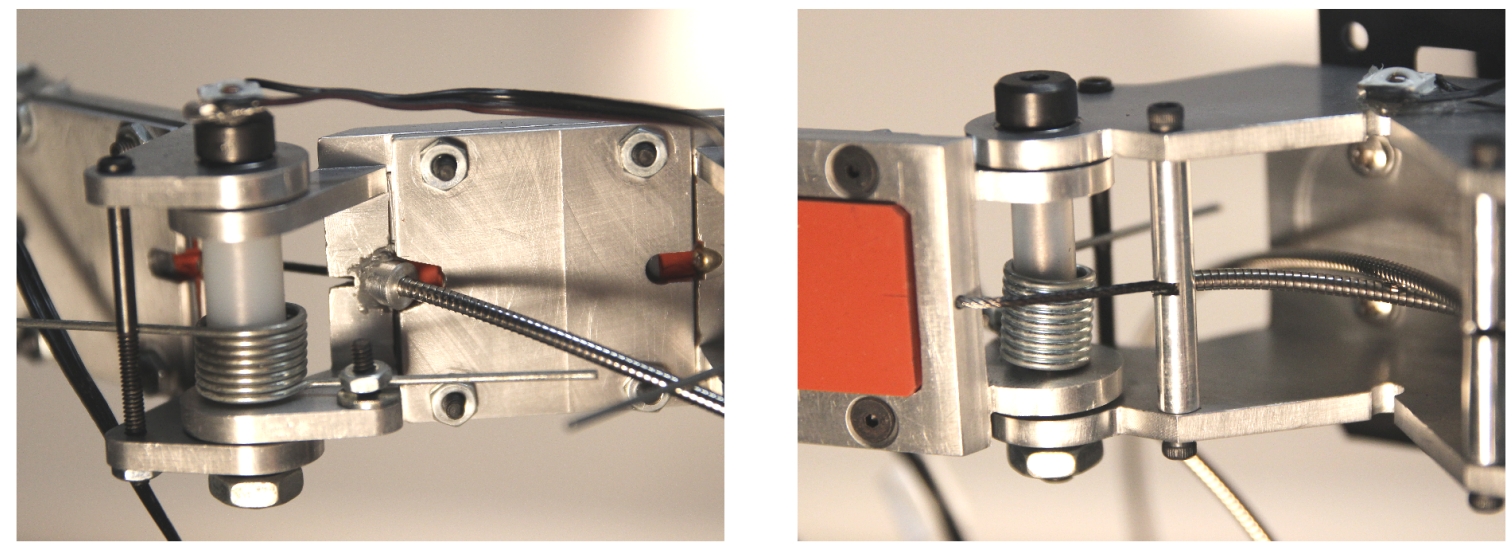

Figure 3.4: Left: friction fit of sheath into the proximal link for the distal joint and torsion spring mounting. Right: friction fit of sheath into the base for the proximal joint.

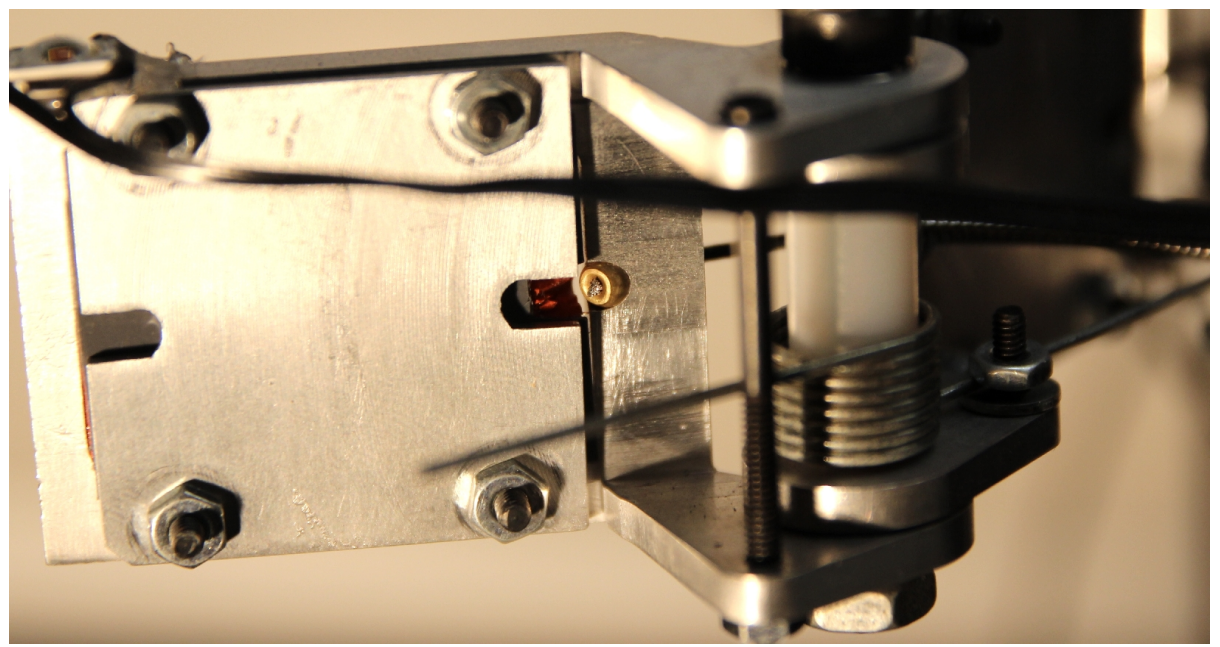

Figure 3.5: Ferrule socket used to attach cable to the pin-off point.

The MX-28 servomotors which apply tension to the cables in the CSTM are contained within the actuator bank; a modular component which mounts to the base of the SPOT platforms below the shoulder joint. The design of the actuator bank is presented in Fig. 3.6, where the four servomotors are mounted within a U-channel. 

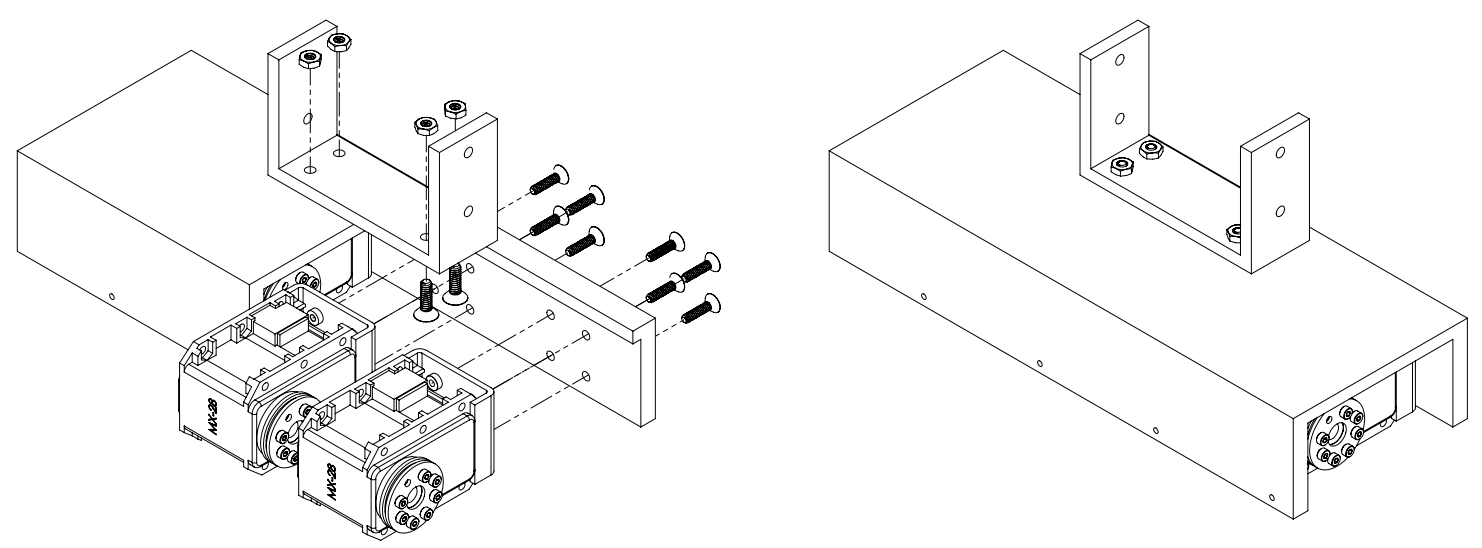

Figure 3.6: Actuator bank design. Exploded view (left) and assembled view (right).

To attach the CSTM to the actuator bank, the sheaths are friction fitted into the four holes on the front of the U-channel such that the cable can be passed through and attached to the winch pulleys. The open end of the cable is clamped between the two pulley plates (see Appendix A.2) screwed into place to ensure there is no pull-out. The installation of the CSTM to the actuator bank is shown by Fig. 3.7 for clarity.

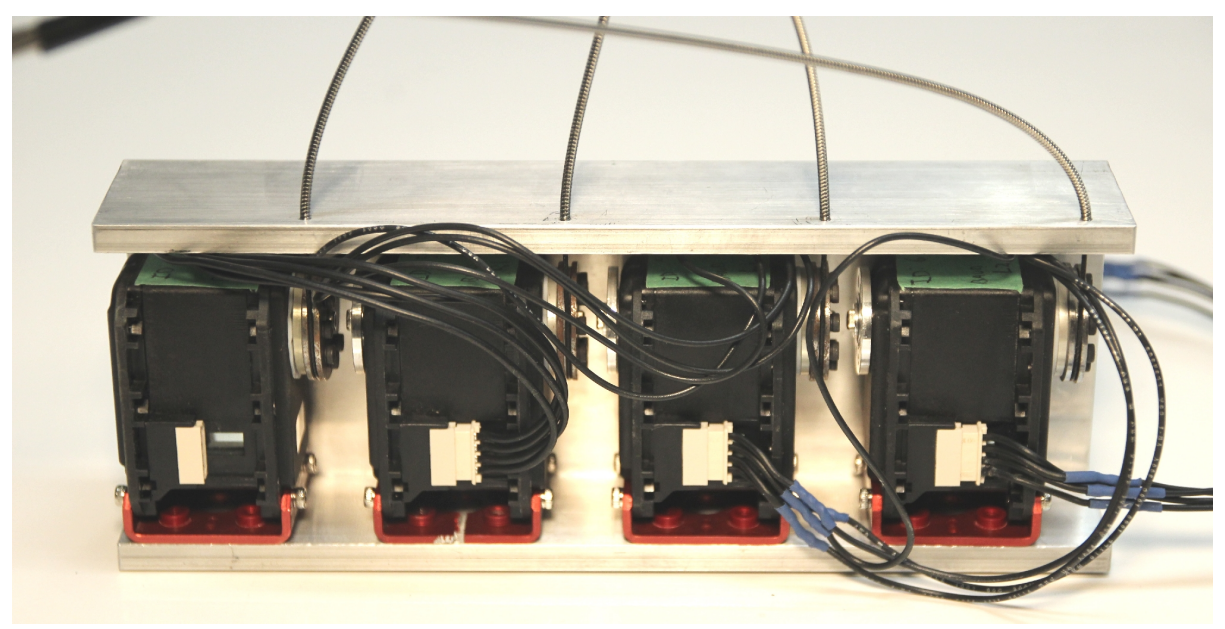

Figure 3.7: CSTM integrated into the actuator bank.

Figure 3.8 highlights the modularity of the fully actuated gripper system, where it can be seen fully installed on the SPOT platform in Fig. 3.9. 


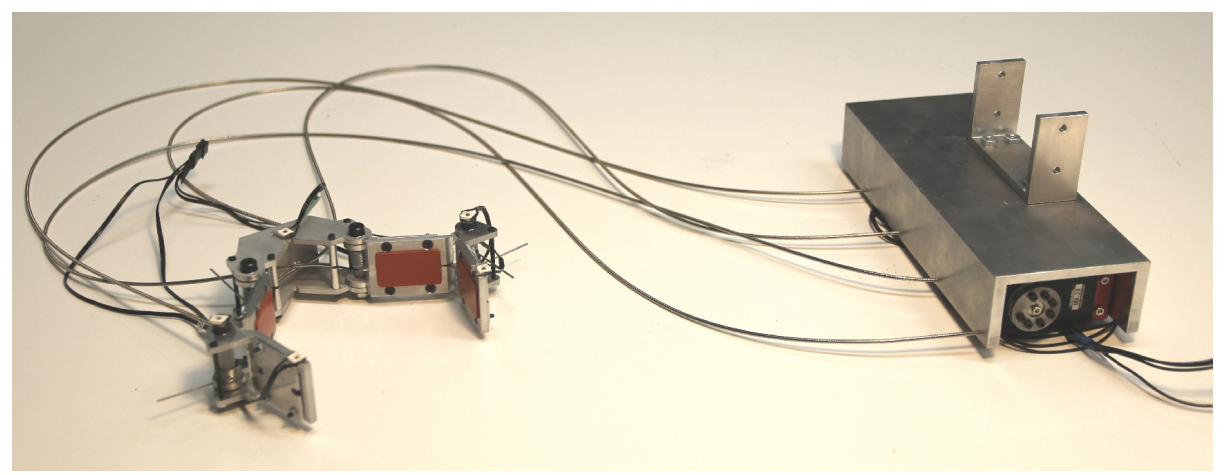

Figure 3.8: Full assembly of the actuated gripper system.

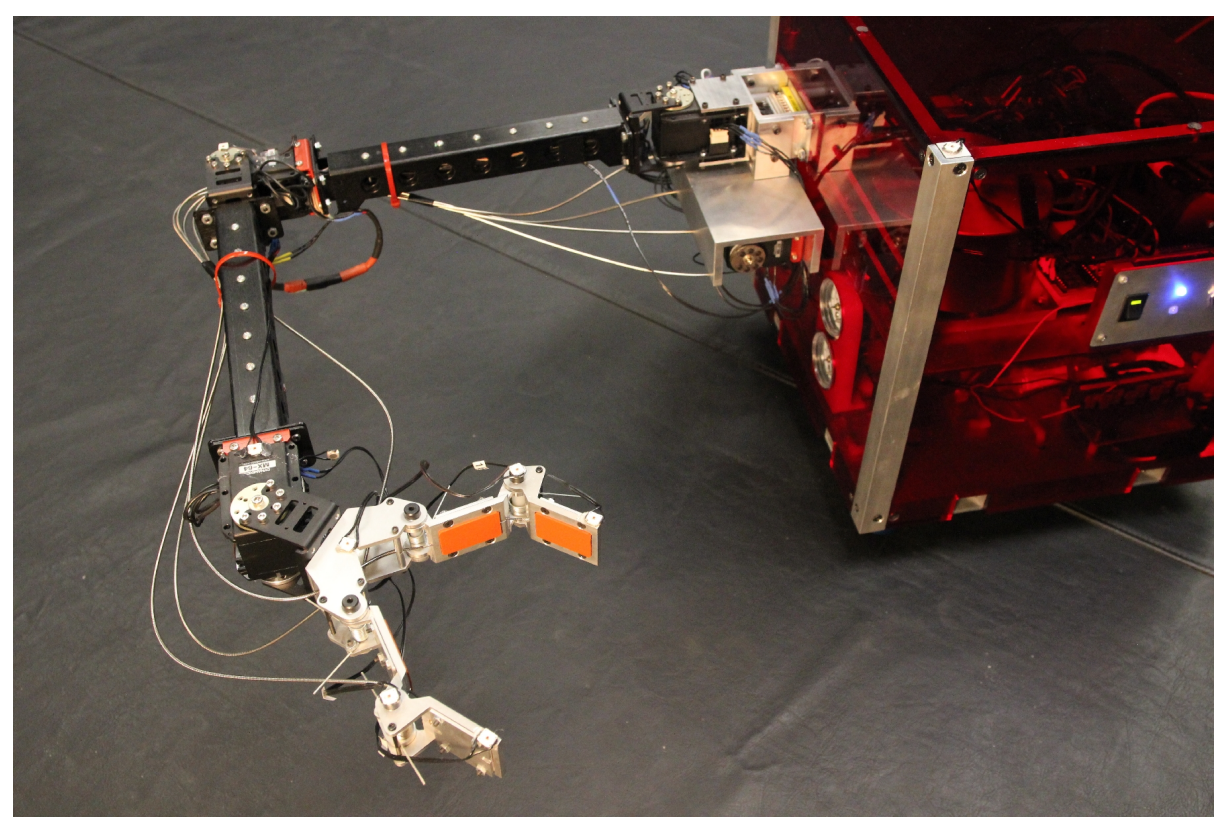

Figure 3.9: Fully integrated gripper onto the SPOT platform.

\subsection{Instrumentation and Measurements}

To extract the joint angles in real time from the gripper, it was deemed most appropriate to utilize the existing ground truth system to extract the position data of the joints from the experiment, and convert them into joint angles by using the inverse kinematics of the manipulator. The primary motivations of choosing the ground truth system over other measurement system, such as encoders, include: cost effectiveness, proven reliability and accuracy from several previous experiments, simplification of 
the joint design, and the simplicity of physical/software implementation in the existing SPOT facilities.

The ground truth system is a PhaseSpace motion capture system. An array of eight stereoscopic cameras track the 3-dimensional Cartesian coordinates of several LED markers with respect to an inertial reference frame; in this case the corner of the gravity offset table. Each LED is pulsing at a unique frequency for marker identification. This motion capture system can determine the position of an LED marker to a resolution of $0.01 \mathrm{~mm}$, which can be measured up to a rate of $240 \mathrm{~Hz}$ by the PhaseSpace server. In the case of this experiment, measurements were set to be taken at a rate of $50 \mathrm{~Hz}$.

The LED configuration on the experimental hardware can be seen in Fig. 3.10, where there are four LEDs attached to the corners of the platform base. These LEDs are used to compute the position and attitude of the base, which is handled by the server directly. The remaining LEDs are attached to the end of each link with respect to the rotational joints.

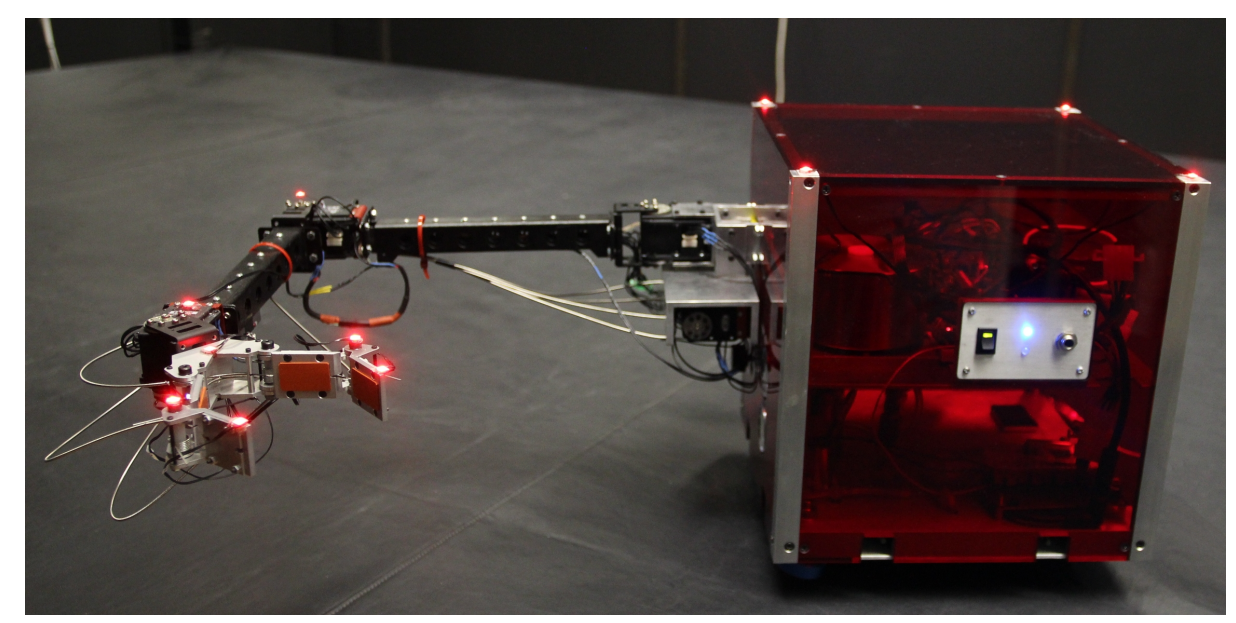

Figure 3.10: Location of LED markers on the SPOT platforms.

In order to convert the position data of these LED markers into joint angles, the inverse kinematics of the SPOT must first be defined. The kinematic model used to derive these equations is presented in Fig. 3.11, where the points marked with a dashed circle are known in the inertial reference frame from the PhaseSpace measurement system. 

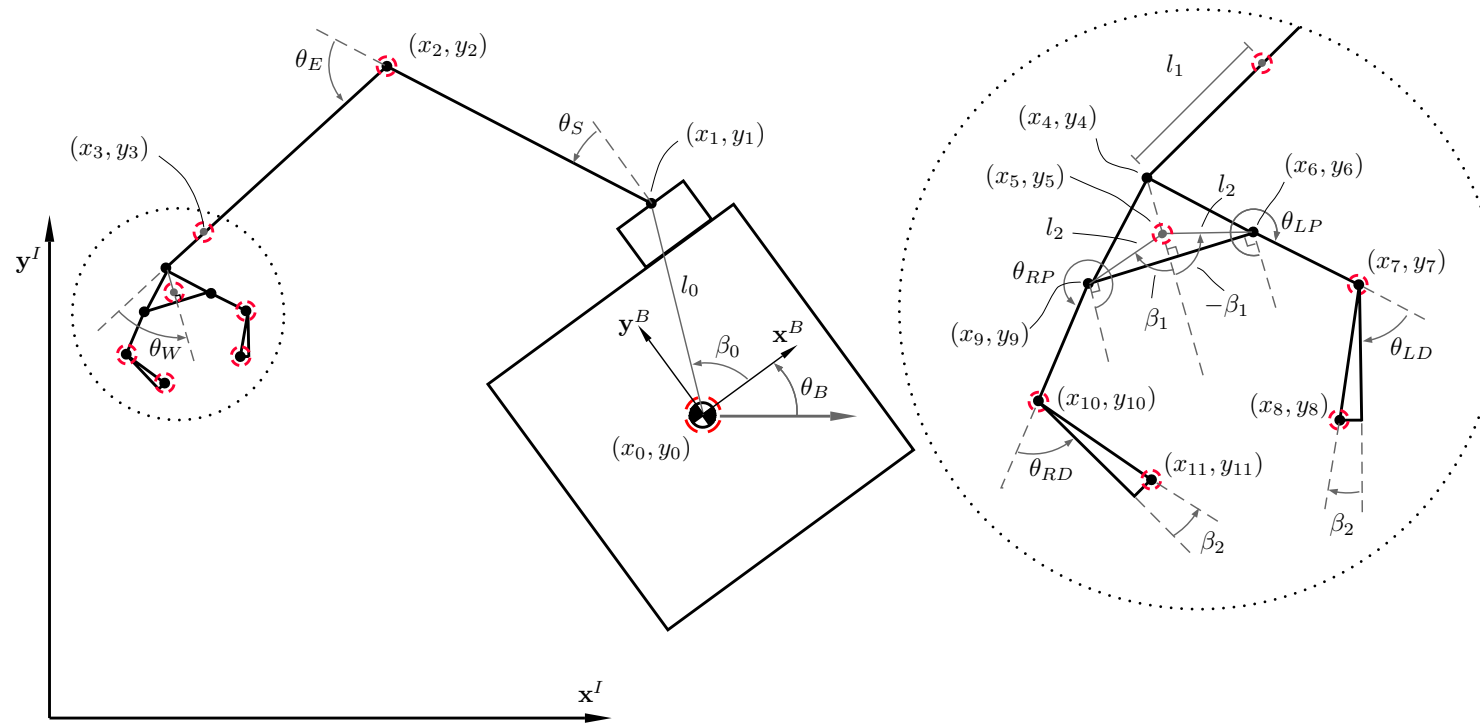

Figure 3.11: Inverse kinematic model of the SPOT platform with arm+gripper.

Note that the joint angles of the arm are measured positive-counter-clockwise, and the gripper joint angles are measured positive with respect to the closing direction. From simple geometry, the joint angle of the arm and gripper can be extracted based on the knowledge of the points $\left(x_{0}, y_{0}\right)$ to $\left(x_{11}, y_{11}\right)$ as per the following relationships.

$$
\begin{aligned}
\theta_{S} & =\arctan \left(\frac{y_{2}-y_{1}}{x_{2}-x_{1}}\right)-\left(\pi / 2+\theta_{B}\right) \\
\theta_{E} & =\arctan \left(\frac{y_{3}-y_{2}}{x_{3}-x_{2}}\right)-\left(\pi / 2+\theta_{B}+\theta_{S}\right) \\
\theta_{W} & =\arctan \left(\frac{y_{5}-y_{4}}{x_{5}-x_{4}}\right)-\left(\pi / 2+\theta_{B}+\theta_{S}+\theta_{E}\right) \\
\theta_{L P} & =-\left(\arctan \left(\frac{y_{7}-y_{6}}{x_{7}-x_{6}}\right)-\left(\pi / 2+\theta_{B}+\theta_{S}+\theta_{E}+\theta_{W}\right)\right) \\
\theta_{L D} & =-\left(\arctan \left(\frac{y_{8}-y_{7}}{x_{8}-x_{7}}\right)-\left(\pi / 2+\theta_{B}+\theta_{S}+\theta_{E}-\theta_{L P}-\beta_{2}\right)\right) \\
\theta_{R P} & =\arctan \left(\frac{y_{10}-y_{9}}{x_{10}-x_{9}}\right)-\left(\pi / 2+\theta_{B}+\theta_{S}+\theta_{E}+\theta_{W}\right) \\
\theta_{R D} & \left.=\arctan \left(\frac{y_{11}-y_{10}}{x_{11}-x_{10}}\right)-\left(\pi / 2+\theta_{B}+\theta_{S}+\theta_{E}+\theta_{R P}+\beta_{2}\right)\right)
\end{aligned}
$$

For the sake of convenience, these equations are wrapped between $-\pi<\theta<\pi$ in 
Table 3.1: List of measured geometric properties.

\begin{tabular}{|c|c|c|}
\hline Parameter & Value & Units \\
\hline \hline$l_{0}$ & 230.4 & $\mathrm{~mm}$ \\
\hline$l_{1}$ & 38.35 & $\mathrm{~mm}$ \\
\hline$l_{2}$ & 34.27 & $\mathrm{~mm}$ \\
\hline$\beta_{0}$ & 68.3 & $\mathrm{deg}$ \\
\hline$\beta_{1}$ & 57.0 & $\mathrm{deg}$ \\
\hline$\beta_{2}$ & 10.0 & $\mathrm{deg}$ \\
\hline
\end{tabular}

the software. Of the points shown, $\left(x_{1}, y_{1}\right),\left(x_{4}, y_{4}\right),\left(x_{6}, y_{6}\right),\left(x_{9}, y_{9}\right)$, are not measured directly and must then be computed based on geometric relationships. From Fig. 3.11, the known parameters include $\beta_{0}, \beta_{1}, \beta_{2}, l_{0}, l_{1}$, and $l_{2}$ based on physical measurements. These values are recorded in Table 3.1.

Using these properties with reference to Eqs. (3.1) to (3.7), the unknown points can be found from the following equations.

$$
\begin{gathered}
{\left[\begin{array}{l}
x_{1} \\
y_{1}
\end{array}\right]=\left[\begin{array}{l}
x_{0}+l_{0} \cos \left(\theta_{B}+\beta_{0}\right) \\
y_{0}+l_{0} \sin \left(\theta_{B}+\beta_{0}\right)
\end{array}\right]} \\
{\left[\begin{array}{l}
x_{4} \\
y_{4}
\end{array}\right]=\left[\begin{array}{l}
x_{3}+l_{1} \cos \left(\pi / 2+\theta_{B}+\theta_{S}+\theta_{E}\right) \\
y_{3}+l_{1} \sin \left(\pi / 2+\theta_{B}+\theta_{S}+\theta_{E}\right)
\end{array}\right]} \\
{\left[\begin{array}{l}
x_{6} \\
y_{6}
\end{array}\right]=\left[\begin{array}{l}
x_{5}+l_{2} \cos \left(\pi / 2+\theta_{B}+\theta_{S}+\theta_{E}+\beta_{1}\right) \\
y_{5}+l_{2} \sin \left(\pi / 2+\theta_{B}+\theta_{S}+\theta_{E}+\beta_{1}\right)
\end{array}\right]} \\
{\left[\begin{array}{l}
x_{9} \\
y_{9}
\end{array}\right]=\left[\begin{array}{l}
x_{5}+l_{2} \cos \left(\pi / 2+\theta_{B}+\theta_{S}+\theta_{E}-\beta_{1}\right) \\
y_{5}+l_{2} \sin \left(\pi / 2+\theta_{B}+\theta_{S}+\theta_{E}-\beta_{1}\right)
\end{array}\right]}
\end{gathered}
$$

Since Eqs. (3.1) through (3.7) depend on having knowledge of the preceding joint angles, they must be executed sequentially (from base to gripper) to be fully defined during the experiments. 


\subsection{Experiment Software}

The SPOT experimental facilities run on software that is developed in the MAT$\mathrm{LAB} /$ Simulink environment, and then is compiled into $\mathrm{C}$ code to run on the on-board computer. The key enabling component that allows the software to communicate with the PhaseSpace measurement system and the Dynamixel MX-28 servo motors is the use of custom hardware access functions. The use of these functions is detailed by Crain [53].

For the purposes of these experiments, there are only two main hardware components that must be integrated into the software. The first being the LED positions from the ground truth system to determine the joint angles as per Section 3.3. The second is having access to the "write" command of the Dynamixel actuators to send position signals from the controllers. To achieve this, the software is set-up as per Fig. 3.12, where the custom hardware access function "Get PhaseSpace Data" acquires the position of all the LED markers, and the "InitializeForArmGripper" block initiates the communication protocol on the Dynamixel servomotors. The remaining features include an array of data-store blocks, the inverse kinematics used to convert the LED positions into joint angles as per Section 3.3, and the initialization logic to begin the experiments 20 seconds after the LEDs have turned on. Within the "If Action Control" block is the main logic for executing the experiment, which is shown in Fig. 3.13. 
Initialize Code Logic

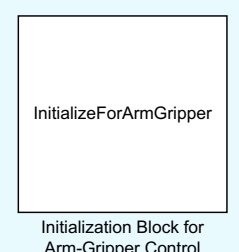

Arm-Gripper Control

Data Store Block Definitions
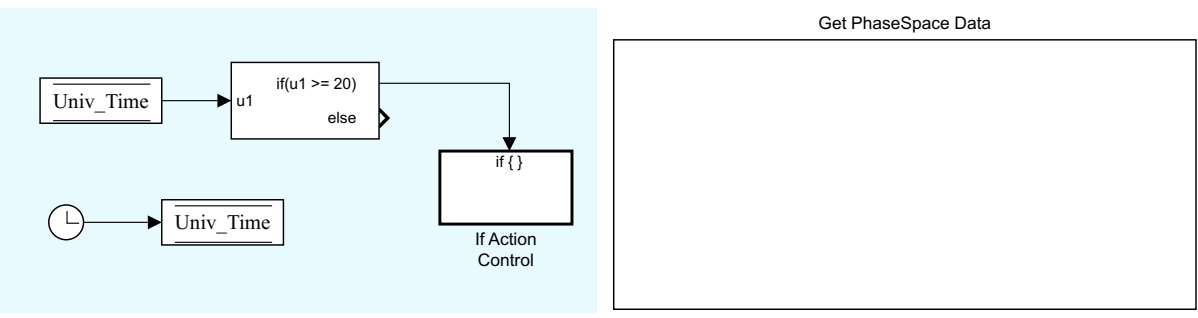

\begin{tabular}{|c|}
\hline ARM_Elbow_Px \\
\hline ARM_Elbow_Py \\
\hline ARM_Wrist_Px \\
\hline GRIPPER_L1_Px \\
\hline GRIPPER_L1_Py \\
\hline GRIPPER_L2_Px \\
\hline
\end{tabular}

\begin{tabular}{|c|c|c|}
\hline RED_Vx & GRIPPER_L1_Rz & GRIPPER_L1_RzD \\
\hline RED_Vy & GRIPPER_L2_Rz & GRIPPER_R1_RzD \\
\hline RED_RzD & GRIPPER_R1_Rz & GRIPPER_L2_RzD \\
\hline GRIPPER_R2_Px & GRIPPER_R2_Rz & GRIPPER_R2_RzD \\
\hline GRIPPER_R2_Py & & \\
\hline Univ_Time & & \\
\hline
\end{tabular}

Inverse Kinematics
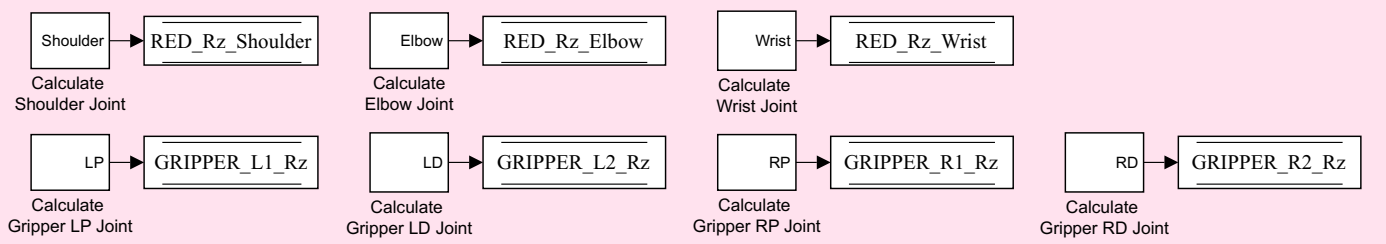

Figure 3.12: Top level Simulink diagram to execute experiments.

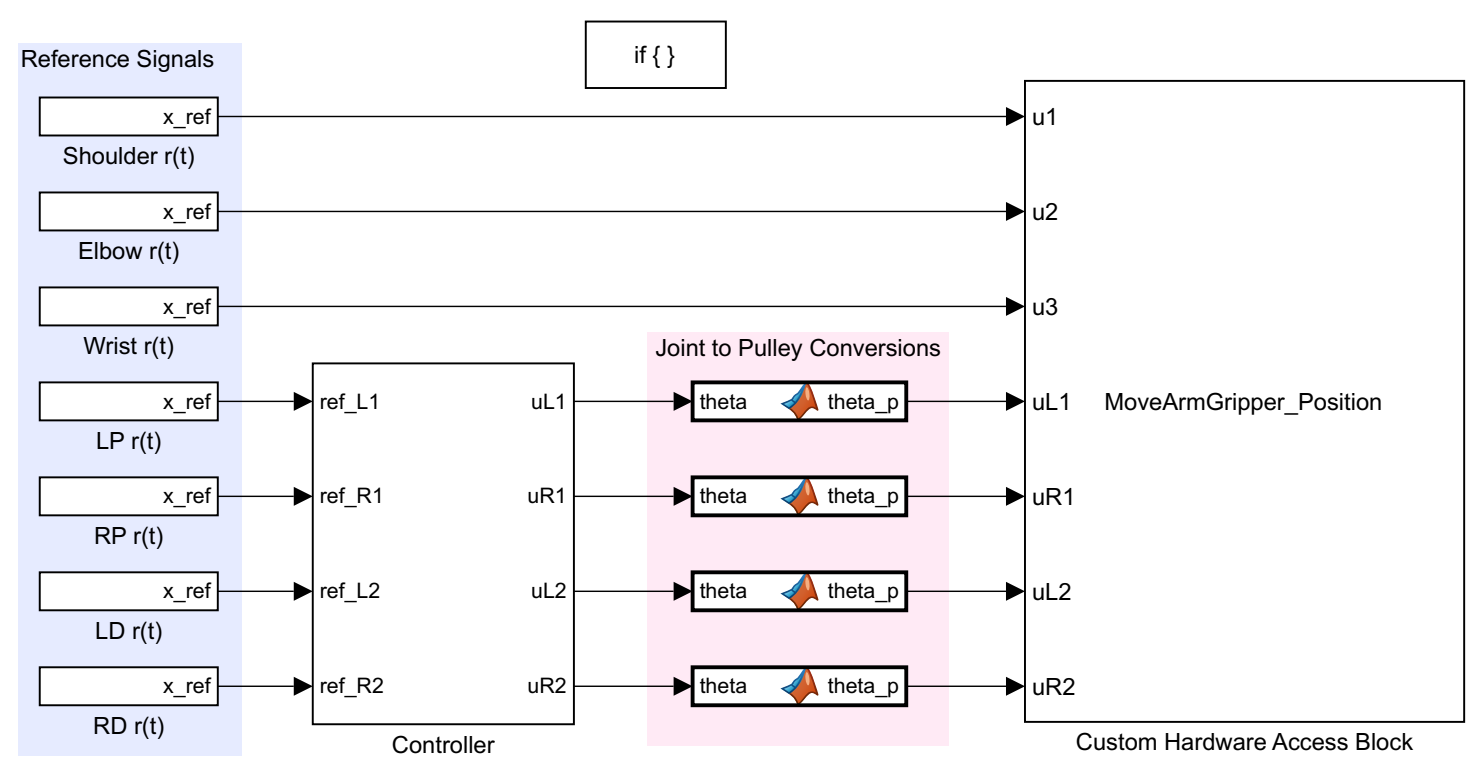

Figure 3.13: Main control logic to drive the experiment. 
The reference signals for the gripper are first sent through the controller block to compute the desired control signal; refer to Chapter 5 for details on the controller implementation. Notice that the output of the controller has an additional conversion applied to it before it is sent to the actuators. This is due to the nature of the nonconstant torque arm at the joints, which is highlighted in Fig. 2.4. In most cases, the output of the CSTM is connected to a pulley which drives the rotation of the joint. This implies that in an ideal CSTM (i.e. no friction), the pulley ratio of input to output is constant. It is clear that this is not the case for this application, so a corrective factor must be applied to relate the commanded actuator input to the joint angle output.

Fortunately, many of the equations in Section 2.2.2 can be used to create a geometric relationship between the desired joint angle, and its corresponding pulley angle at the input. To do so, let us define the change tendon displacement at the input as $\Delta l_{p}$ (winch pulley), and $\Delta l_{j}$ at the output (joint). Since the winch has a constant radius, $\Delta l_{p}$ is simply the arc length that the pulley has rotated a displacement of $\theta_{m}$ from its maximum angle $\theta_{m_{\max }}$.

$$
\Delta l_{p}=\left(r_{p}+r_{c}\right)\left(\theta_{m}-\theta_{m_{\max }}\right)
$$

where $r_{p}$ is the radius of the winch pulley, and $r_{c}$ is the radius of the cable. For the joint cable displacement, Eq. (2.8) can be used directly by taking the difference of the exposed tendon length at the desired joint angle $\theta_{j}$, and the minimum tendon length (which occurs at the maximum joint angle $\theta_{j_{\max }}$ ).

$$
\Delta l_{j}=L_{e}\left(\mathbf{x}_{j}, \theta_{j}\right)-L_{e}\left(\mathbf{x}_{j}, \theta_{j_{\max }}\right)
$$

Note that this expression is generalized for each joint, where $\mathbf{x}_{j}$ is the sheath and tendon placement at the joint of interest (see Table 2.1). For an ideal CSTM with no friction, the change in tendon length should be equivalent such that $\Delta l_{m}=\Delta l_{j}$. Thus, by combining Eq. (3.12) and Eq. (3.13), the required pulley angle for a desired joint angle $\theta_{j}$ can be determined as follows. 


$$
\theta_{m}=\frac{L_{e}\left(\mathbf{x}_{j}, \theta_{j}\right)-L_{e}\left(\mathbf{x}_{j}, \theta_{j_{\max }}\right)}{r_{p}+r_{c}}+\theta_{m_{\max }}
$$

With this conversion implemented in the user-defined function blocks for each joint, the control signal is then converted into the required actuator angle $\theta_{p}$ and then sent to the custom hardware access block "MoveArmGripper_Position". This block establishes communication with the Dynamixel actuators, and sends the signal as a goal position for its internal PID control logic (see Fig. 3.14), where the PID gains and the angular limiter can be adjusted.

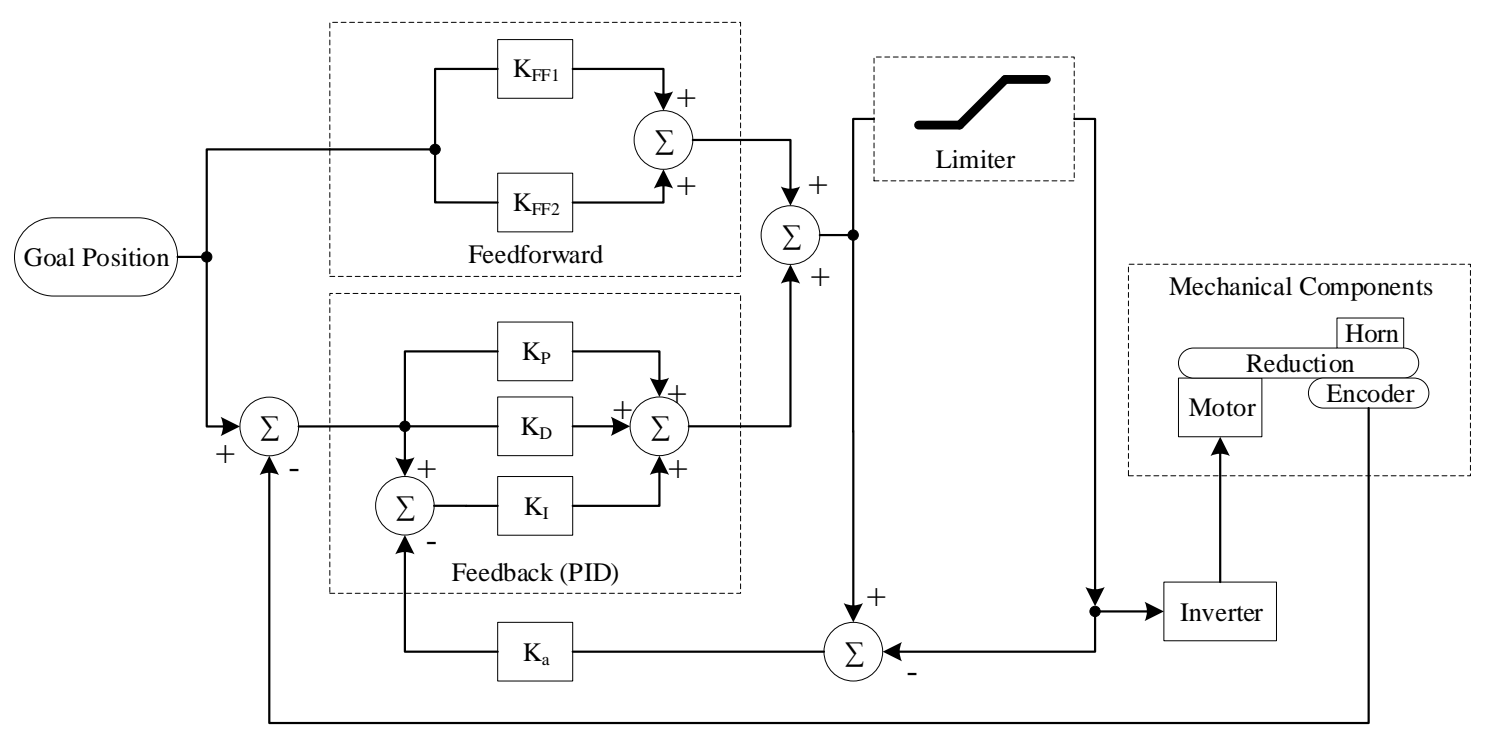

Figure 3.14: Internal Dynamixel PID control logic [53].

The PID gains selected were $K_{P}=500, K_{I}=25$, and $K_{D}=500$ for the arm, and $K_{P}=1000, K_{I}=25$, and $K_{D}=50$ for the gripper actuators by trial and error to ensure smooth motion of the manipulator with minimal overshoot. These gains were held constant across all experiments to maintain continuity. 


\section{Chapter 4}

\section{Adaptive Control Strategies for CSTMs}

This chapter explores the system description of the CSTM in context to the control problem, and the theory of the proposed control strategies to be implemented in both simulation and experiment. Specifically, Section 4.1 covers the system architecture in the context of the proposed control problem. Next, Sections 4.3 and 4.2 cover the theory of the $\mathcal{L}_{1}$ controller and the indirect adaptive controller developed by Do et al. $[45,46]$ respectively.

\subsection{System Description and Control Strategies}

Before the specifics of the selected control strategies are considered, let us first review the control objectives for the gripper. First, we define the states of each gripper joint to be the angular position and velocity of each individual link.

$$
\mathbf{x}_{i}=\left[\begin{array}{c}
\theta_{i} \\
\dot{\theta}_{i}
\end{array}\right]
$$

where the subscript $i$ can be $L P, R P, L D$, and $R D$ for the right proximal, left proximal, right distal, and left distal joints, respectively. Since each link is controlled by an independent actuator with its own CSTM and unique joint geometry, it was deemed appropriate to implement an individual control loop for each joint separately. The general control loop for a given joint is presented by Fig. 4.1, where $\mathbf{u}(t)$ is the control signal based on state feedback sent to the MX-28 servomotor, which drives the CSTM to actuate the joint. 


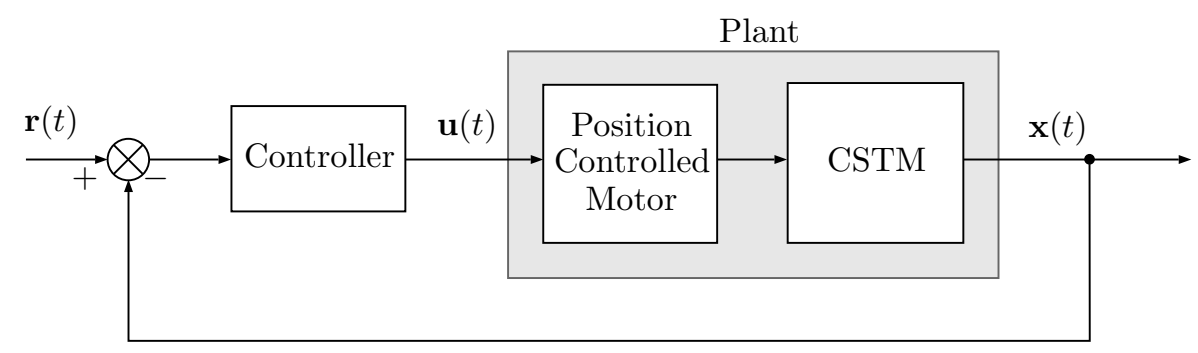

Figure 4.1: General control architecture for the CSTM driven gripper.

The exact control architecture may change based on the control algorithms selected, however, the principle of the control objective will remain the same: implement a state-feedback type controller to command a desired joint angle trajectory.

As it was mentioned previously in Section 1.3.2, CSTMs used in robotic manipulators have many desirable characteristics. However, due to the transmission characteristics of CSTMs, they are rather challenging to implement from the controls perspective due to friction and backlash hysteresis that is present in the system. This would imply that the set point tracking accuracy for a fixed controller would vary as a function of the CSTM configuration at any given time. Therefore, it was deemed appropriate to employ two adaptive control strategies to compare against the uncontrolled case and a standard fixed-gain PID controller. The control laws selected for this application include the system specific indirect controller developed for CSTMs by Do et al. $[45,46]$, and the direct $\mathcal{L}_{1}$ controller, which has not been applied to a CSTM before.

\subsection{Indirect Adaptive Control from Modified Bouc-Wen Hysteresis Model}

In the case of the indirect control method developed by Do et al. [45,46], the algorithm makes use of the modified Bouc-Wen kinematic model for CSTMs (hereinafter referred to as the $\mathrm{AB}$ controller). Therefore, this section will cover the mathematical form of the CSTM model required (Section 4.2.1), and the derivation of the AB control algorithm (Section 4.2.2). 


\subsubsection{CSTM Kinematic Model}

As it was shown by Do et al. [44], any CSTM can be defined by using a non-linear backlash hysteresis model for a fixed configuration. This model was adapted from the generalized asymmetric Bouc-Wen model developed by Song [55], which was optimized for computational efficiency by reducing the number of non-linear parameters while giving it a continuous form. This model is able to determine the output position of a fixed CSTM configuration given the position and velocity of the tendon at the input end of the sheath. The model is given by Eq. (4.2), where $x_{i n}$ represents the position of the tendon at the input, and $x_{\text {out }}$ represents the position of the tendon at the output end of the sheath.

$$
x_{\text {out }}(t)=\alpha_{x}\left(\dot{x}_{i n}(t)\right) x_{i n}(t)+\zeta(t)
$$

In this expression, the function $\alpha_{x}\left(\dot{x}_{i n}(t)\right)$ is a smooth transition function between loading and unloading phases of the hysteresis loop, and $\zeta(t)$ is the internal state variable of the CSTM. These parameters can be determined by Eq. (4.3) and Eq. (4.4) respectively.

$$
\begin{gathered}
\alpha_{x}\left(\dot{x}_{i n}(t)\right)=\frac{\alpha_{1} e^{2 \dot{x}_{i n}(t)}+\alpha_{2}}{e^{2 \dot{x}_{i n}(t)}+1} \\
\dot{\zeta}(t)=A \dot{x}_{i n}(t)-\nu\left|\dot{x}_{i n}(t)\right| \zeta(t)+\delta\left|\dot{x}_{i n}(t)\right|
\end{gathered}
$$

where the parameters $\alpha_{1}>0, \alpha_{2}>0, A, \nu$, and $\delta$ are all constant parameters which control the shape of the hysteresis loop for a fixed configuration. In practice, these parameters would be determined for a single configuration of the CSTM by recording experimental data and performing any form of parameter estimation technique (i.e. optimization routine, genetic algorithm, etc.,) to return the model values that best fit the data. 


\subsubsection{AB Control Algorithm}

With the reference model outlined in Section 4.2.1, it can be restructured as a controlled system by recognizing that $x_{i n}(t)$ is the input to the system and consider it as the control input $u(t)$ to the CSTM. Additionally, we introduce an unknown disturbance $d(t)$ on the internal state $\zeta(t)$ of the system (Eq. (4.2)) to represent a sudden configuration change to the CSTM.

$$
\begin{aligned}
\Phi(t) & =\alpha_{x}(\dot{u}(t)) u(t)+\zeta(t)+d(t) \\
& =\alpha_{x}(\dot{u}(t)) u(t)+\Delta_{n}(t)
\end{aligned}
$$

where $\Phi(t)$ is the output of the system, and $\Delta_{n}(t)$ is the internal state combined with the disturbance bounded by $\Delta_{n}^{*}(t)$, which is a positive value. Before the AB control algorithm is developed, the following assumptions must be made: 1), the cable is under tension at all times and 2), the exact model parameters of the CSTM and their bounds are unknown and are not required for the operation of the controller.

The objective of the controller is to have $\Phi(t)$ track a known reference position signal $x_{r}(t)$, where the tracking error between them is defined to be $e_{r}(t)=\Phi(t)-$ $x_{r}(t)$. With this definition, the following coordinate transformations for Eq. (4.6) can be defined.

$$
\begin{gathered}
n(t)=\int_{0}^{t} e_{r}(\tau) d \tau \\
\omega(t)=e_{r}(t)+\alpha n(t)
\end{gathered}
$$

where $n(t)$ is the cumulative integral of the tracking error (or error history), $\alpha$ is a user defined scaling factor, and $\omega(t)$ can be is a linear combination of the error and its integral. Therefore, $\omega(t)$ is analogous to a proportional-integral controller applied to the error signal of the system. By taking the derivative of this term, and by making use of Eqs. (4.3), (4.6), and (4.8), $\dot{\omega}(t)$ can be re-written as the following. 


$$
\begin{gathered}
\dot{\omega}(t)=\alpha e_{r}(t)+\dot{e}_{r}(t) \\
\dot{\omega}(t)=\alpha\left[\left(\frac{\alpha_{1} e^{2 \dot{u}(t)}+\alpha_{2}}{e^{2 \dot{u}(t)}+1}\right) u(t)+\Delta_{n}(t)-x_{r}(t)\right]+\dot{e}_{r}(t)
\end{gathered}
$$

To simplify the controller design, $u(t)$ can be defined as the linear combination of two separate controllers $u_{1}(t)$ and $u_{2}(t)$ such that Eq. (4.11) is valid.

$$
u(t)=u_{1}(t)+u_{2}(t)
$$

where $u_{1}(t)$ and $u_{2}(t)$ are defined as follows.

$$
\begin{aligned}
& u_{1}(t) \triangleq \frac{u e^{2 u(t)}}{e^{2 \dot{u}(t)}+1} \\
& u_{2}(t) \triangleq \frac{u(t)}{e^{2 \dot{u}(t)}+1}
\end{aligned}
$$

Upon careful inspection, one can conclude that the term in Eq. (4.10) due to $\alpha_{x}(\dot{u}(t)) u(t)$ is equivalent to the linear combination of $u_{1}(t)$ and $u_{2}(t)$ as demonstrated below.

$$
\alpha_{1} u_{1}(t)+\alpha_{2} u_{2}(t)=\frac{\alpha_{1} e^{2 \dot{u}(t)}+\alpha_{2}}{e^{2 \dot{u}(t)}+1} u(t)
$$

Combining Eq. (4.14) reduces Eq. (4.10) to a simpler form.

$$
\dot{\omega}(t)=\alpha\left[\alpha_{1} u_{1}(t)+\alpha_{2} u_{2}(t)+\Delta_{n}(t)-x_{r}(t)\right]+\dot{e}_{r}(t)
$$

For later convenience, let $\chi_{i}(i=1,2)$ be the inverse of $\alpha_{i}$, and let $\Delta_{n}(t)$ be redefined in terms of its boundary $\Delta_{n}^{*}(t)$, as provided in Eq. (4.16).

$$
\Delta_{n}(t)=\tanh \left(\frac{\omega(t)}{\epsilon}\right) \Delta_{n}^{*}(t)
$$

By substituting these definitions back into Eq. (4.15), the expression for $\dot{\omega}(t)$ becomes the following. 


$$
\dot{\omega}(t)=\alpha\left[\frac{1}{\chi_{1}} u_{1}(t)+\frac{1}{\chi_{2}} u_{2}(t)+\tanh \left(\frac{\omega(t)}{\epsilon}\right) \Delta_{n}^{*}(t)-x_{r}(t)\right]+\dot{e}_{r}(t)
$$

Since $\chi_{1}, \chi_{2}$, and $\Delta_{n}^{*}(t)$ are unknown parameters, they are replaced with their respective estimated parameters which are assumed to be ideal.

$$
\dot{\omega}(t)=\alpha\left[\frac{1}{\hat{\chi}_{1}} u_{1}(t)+\frac{1}{\hat{\chi}_{2}} u_{2}(t)+\tanh \left(\frac{\omega(t)}{\epsilon}\right) \hat{\Delta}_{n}^{*}(t)-x_{r}(t)\right]+\dot{e}_{r}(t)
$$

Equation 4.18 is then further simplified by expressing $u_{i}(t)$ in terms of virtual controllers $\bar{u}_{i}(t)$, where by definition $u_{i}(t)=\hat{\chi}_{i} \bar{u}_{i}(t)$.

$$
\dot{\omega}(t)=\alpha\left[\bar{u}_{1}(t)+\bar{u}_{2}(t)+\tanh \left(\frac{\omega(t)}{\epsilon}\right) \hat{\Delta}_{n}^{*}(t)-x_{r}(t)\right]+\dot{e}_{r}(t)
$$

Since $\dot{\omega}(t)$ is related to the error signal $e_{r}(t), \bar{u}_{i}$ is selected such that Eq. (4.19) can be expressed in a similar form to Eq. (4.9) while cancelling out unnecessary terms. Therefore, the virtual controllers are designed as per the following expressions.

$$
\begin{aligned}
& \bar{u}_{1}(t)=-k_{1} \omega(t)-\tanh \left(\frac{\omega(t)}{\epsilon}\right) \hat{\Delta}_{n}^{*}(t)+x_{r}(t)-\frac{1}{\alpha} \dot{e}_{r}(t) \\
& \bar{u}_{2}(t)=-k_{2} \omega(t)
\end{aligned}
$$

where $k_{1}$ and $k_{2}$ are positive proportional constants used to control the weight of each virtual controller. Recalling the definition of $u(t)$ from Eq. (4.11), the final form of the controller can be constructed as per Eq. (4.22). Note that $\hat{\chi}_{i}$ will vary with time for a changing CSTM configuration.

$$
u(t)=\hat{\chi}_{1}(t) \bar{u}_{1}(t)+\hat{\chi}_{2}(t) \bar{u}_{2}(t)
$$

The adaptive laws to determine the estimated parameters $\hat{\chi}_{1}(t), \hat{\chi}_{2}(t)$, and $\hat{\Delta}_{n}^{*}(t)$ are given in Eqs. (4.23) to (4.25), where $\delta_{i}$ is a user specified positive constant to control the adaptation rate, and $\sigma_{i}$ is a small positive constant used to prevent the estimated 
parameters from diverging to large values.

$$
\begin{aligned}
& \dot{\hat{\chi}}_{1}(t)=-\delta_{1} \bar{u}_{1}(t) \omega(t)-\sigma_{1} \hat{\chi}_{1}(t) \\
& \dot{\hat{\chi}}_{2}(t)=-\delta_{2} \bar{u}_{2}(t) \omega(t)-\sigma_{2} \hat{\chi}_{1}(t) \\
& \dot{\hat{\Delta}}_{n}^{*}(t)=\delta_{3} \omega(t) \tanh \left(\frac{\omega(t)}{\epsilon}\right)-\sigma_{3} \hat{\Delta}_{n}^{*}(t)
\end{aligned}
$$

Theorem 1. Consider the non-linear system outlined in Eq. (4.10), and the adaptive controller given by Eqs. (4.20) to (4.25).

1. The position tracking error $e_{r}(t)$ and the adaptive parameters $\hat{\chi}_{1}(t), \hat{\chi}_{2}(t)$, and $\hat{\Delta}_{n}^{*}(t)$ are uniformly ultimately bounded in the closed-loop system.

2. The position tracking error $e_{r}(t)$ converges to a desired compact region in the presence of unknown model parameters.

Proof. Please refer to the work of Do et al. $[45,46]$ for the Lyapunov stability proof of this theorem.

\section{3 $\mathcal{L}_{1}$ Control with Hysteresis Compensation}

There are several $\mathcal{L}_{1}$ adaptive control strategies available in literature which have been shown to be effective for a many types of systems. In the case of a system with hysteresis, such as the implemented CSTM, it has been shown by multiple authors that the $\mathcal{L}_{1}$ controller with a state predictor and uncertain input gains is an appropriate choice [56-58].

This section covers the system identification and definition of the gripper in Section 4.3.1, the development of the companion model adaptive controller (CMAC) with a state predictor in Section 4.3.2, and the modification of the CMAC architecture into the $\mathcal{L}_{1}$ control architecture in Section 4.3.3. 


\subsubsection{System Description}

In CMAC control, it is necessary that the plant is a linear time invariant, single-input single-output (SISO) system governed by the following state space formulation.

$$
\begin{aligned}
& \dot{\mathbf{x}}(t)=\mathbf{A} \mathbf{x}(t)+\mathbf{B u}(t) \\
& \mathbf{y}(t)=\mathbf{C x}(t)
\end{aligned}
$$

where $\mathbf{x}(t)$ represents the $n \times 1$ state vector of the system, $\mathbf{u}(t)$ is the $n \times 1$ control input, and $\mathbf{y}(t)$ is the output of the system, $\mathbf{A}$ is the $n \times n$ system matrix, and $\mathbf{B}$ is the $n \times m$ control matrix. However, since CSTM systems are highly non-linear hysteretic systems, their dynamics do not follow Eq. (4.26) and Eq. (4.27). Fortunately, it has been demonstrated that such systems can be controlled with a modified $\mathcal{L}_{1}$ control architecture, where the hysteresis non-linearities are treated as an uncertain system input gain [56-59]. This can be shown graphically in Fig. 4.2 as a decoupled system with the hysteresis transformation occurring before the plant.

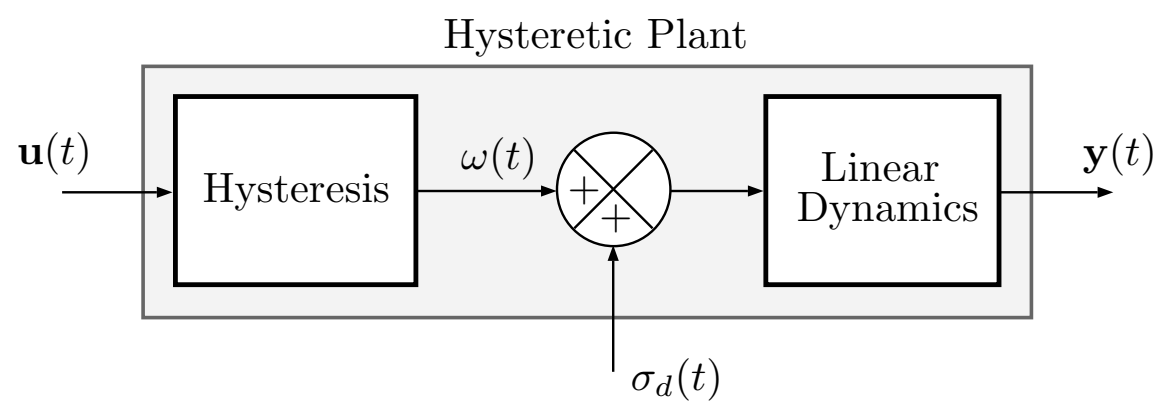

Figure 4.2: Decoupled hysteresis model and plant for non-linear system.

where the signal $\boldsymbol{\omega}(t)$ is the transformed control signal, and $\boldsymbol{\sigma}_{d}(t)$ is a vector of bounded disturbances such as noise and other perturbations. By applying these signals to the definition of the dynamics presented by Eq. (4.26), the system dynamics can then be rewritten as per Eq. (4.28) below.

$$
\dot{\mathbf{x}}(t)=\mathbf{A x}(t)+\mathbf{B}\left(\boldsymbol{\omega}_{u}(t)+\boldsymbol{\sigma}_{d}(t)\right)
$$


The hysteresis transformation $\boldsymbol{\omega}_{u}(t)$ is found by applying Eq. (4.29) to the bounded control signal $\mathbf{u}(t)$.

$$
\boldsymbol{\omega}_{u}(t)=\gamma(t) \mathbf{u}(t)+\boldsymbol{\sigma}_{u}(t)
$$

where the definitions of $\gamma(t)$ and $\boldsymbol{\sigma}_{u}(t)$ are best described by referring to the graphical representation of the hysteresis transformation shown in Fig. 4.3 below, where $\boldsymbol{\omega}_{u}(t)$ and $\mathbf{u}(t)$ are mapped to the vertical and horizontal axes respectively.

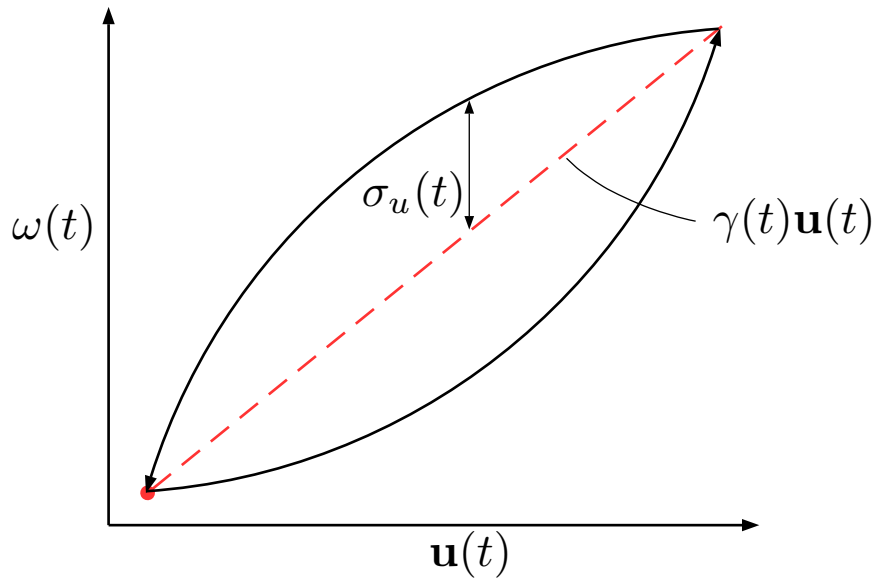

Figure 4.3: Graphical representation of the non-linear transformation applied to the system input $\mathbf{u}(t)$.

By substituting Eq. (4.29) into Eq. (4.28), and by defining $\boldsymbol{\sigma}(t)=\boldsymbol{\sigma}_{d}(t)+\boldsymbol{\sigma}_{u}(t)$, the state equation of the system can be found by Eq. (4.30) below.

$$
\dot{\mathbf{x}}(t)=\mathbf{A x}(t)+\mathbf{B}(\gamma(t) \mathbf{u}(t)+\boldsymbol{\sigma}(t))
$$

For a real system, it can be assumed that the parameters $\gamma(t)$ and $\boldsymbol{\sigma}(t)$ are uniformly bounded, and that there is partial knowledge of the system as per Eq. (4.31) and Eq. (4.32).

$$
\gamma(t) \in \Upsilon_{0} \triangleq\left[\gamma_{l}, \gamma_{u}\right]
$$




$$
|\boldsymbol{\sigma}(t)| \leq \Delta_{o} \quad \forall t \geq 0
$$

where $0 \leq \gamma_{l} \leq \gamma_{u}$, and $\Delta_{o}$ are known/user-defined constants which define the conservative bounds for their respective parameters.

\subsubsection{CMAC Architecture}

Now that the system to be controlled has been defined, let us define a control objective based on the standard CMAC architecture. At its most fundamental level, the control objective of CMAC is to design an adaptive feedback control signal $\mathbf{u}(t)$ which minimizes the error between the output of the system $\mathbf{y}(t)$ and a user defined reference model. This reference model is defined by Eqs. (4.33) and (4.34), which determines the desired closed-loop dynamics in which the real system should follow.

$$
\begin{aligned}
& \dot{\mathbf{x}}_{m}(t)=\mathbf{A}_{m} \mathbf{x}_{m}(t)+\mathbf{B} k_{g} \mathbf{r}(t) \\
& \mathbf{y}_{m}(t)=\mathbf{C x}(t)
\end{aligned}
$$

In this model, $\mathbf{A}_{m}$ is a known Hurwitz matrix of the desired dynamics defined by the user, $\mathbf{B}$ is the control matrix of the system, and $\mathbf{r}(t)$ is the reference signal known a priori. The matrix $\mathbf{A}_{m}$ is considered Hurwitz if and only if every eigenvalue of $\mathbf{A}_{m}$ has a strictly negative real part. The scalar $k_{g}$ is defined by Eq. (4.35) such that the output of the system will converge to the reference signal as $t \rightarrow \infty$.

$$
k_{g} \triangleq-\frac{1}{\mathbf{C A}_{m}^{-1} \mathbf{B}}
$$

By definition, the relationship to the actual system matrix $\mathbf{A}$ and the companion

matrix $\mathbf{A}_{m}$ is defined by Eq. (4.36), where $\boldsymbol{\theta}(t)=\left[\theta_{1}, \ldots, \theta_{n}\right]^{T}$ is a set of unknown parameters assumed to be uniformly bounded and belonging to the convex compact set $\boldsymbol{\theta}(t) \in \boldsymbol{\Theta}$.

$$
\mathbf{A} \triangleq \mathbf{A}_{m}+\mathbf{B} \boldsymbol{\theta}(t)^{T}
$$


The system matrix A outlined previously in Eq. (4.30) is typically unknown. However, with the relationship given in Eq. (4.36), the system can now be more conveniently expressed in terms of the companion matrix $\mathbf{A}_{m}$, as shown in Eq. (4.37).

$$
\dot{\mathbf{x}}(t)=\mathbf{A}_{m} \mathbf{x}(t)+\mathbf{B}\left(\gamma(t) \mathbf{u}(t)+\boldsymbol{\theta}(t)^{T} \mathbf{x}(t)+\boldsymbol{\sigma}(t)\right)
$$

By assuming the same form of the redefined system model in Eq. (4.37), the state predictor in Eq. (4.38) is defined, where the the unknown parameters are replaced with their estimated values, $\hat{\gamma}(t), \hat{\boldsymbol{\theta}}(t)$, and $\hat{\boldsymbol{\sigma}}(t)$ accordingly.

$$
\dot{\hat{\mathbf{x}}}(t)=\mathbf{A}_{m} \hat{\mathbf{x}}(t)+\mathbf{B}\left(\hat{\gamma}(t) \mathbf{u}(t)+\hat{\boldsymbol{\theta}}(t)^{T} \mathbf{x}(t)+\hat{\boldsymbol{\sigma}}(t)\right)
$$

The projection adaptation laws used to govern the estimated parameters are given by Eqs. (4.39) to (4.41), where $\tilde{\mathbf{x}}(t)=\hat{\mathbf{x}}(t)-\mathbf{x}(t)$ is the parametric estimation error dynamics by definition.

$$
\begin{aligned}
& \dot{\hat{\gamma}}(t)=\Gamma \operatorname{Proj}(\hat{\gamma}(t),-\tilde{\mathbf{x}}(t) \mathbf{P B u}(t)) \\
& \dot{\hat{\boldsymbol{\theta}}}(t)=\Gamma \operatorname{Proj}(\hat{\boldsymbol{\theta}}(t),-\tilde{\mathbf{x}}(t) \mathbf{P B} \mathbf{x}(t)) \\
& \dot{\hat{\boldsymbol{\sigma}}}(t)=\Gamma \operatorname{Proj}(\hat{\boldsymbol{\sigma}}(t),-\tilde{\mathbf{x}}(t) \mathbf{P B})
\end{aligned}
$$

In these adaptive laws, $\Gamma$ is the learning rate of the control algorithm, and the matrix $\mathbf{P}=\mathbf{P}^{T}>0$ is the solution to the Lyapunov equation $\mathbf{A}_{m}^{T} \mathbf{P}+\mathbf{P} \mathbf{A}_{m}=-\mathbf{Q}$ for an arbitrary $\mathbf{Q}=\mathbf{Q}^{T}>0$. The projection operation used in these adaptation laws is outlined by Hovakimyan and Cao [59].

With this information, a nominal controller $\mathbf{u}_{n}(t)$ can be defined such that the effects of the unknown parameters are cancelled out, and that the system dynamics become the reference model outlined in Eq. (4.33), implying perfect set-point tracking. By letting $\boldsymbol{\eta}(t) \triangleq \gamma(t) \mathbf{u}(t)+\boldsymbol{\theta}(t)^{T} \mathbf{x}(t)+\boldsymbol{\sigma}(t)$, the nominal controller can be designed as per Eq. (4.42).

$$
\mathbf{u}_{n}(t)=-\boldsymbol{\eta}(t)+k_{g} \mathbf{r}(t)
$$


Unfortunately, as previously stated, the parameters contained within $\boldsymbol{\eta}(t)$ are unknown, so nominal control performance cannot be achieved. Thus, the controller must make use of the estimated parameters, resulting in the final form of the control law in Eq. (4.43).

$$
\mathbf{u}(t)=-\hat{\boldsymbol{\eta}}(t)+k_{g} \mathbf{r}(t)
$$

One of the key properties of the CMAC algorithm is that the tracking error between the companion system and the plant can be shown to converge to zero as one increases adaptation rate.

$$
\|\tilde{\mathbf{x}}(t)\| \leq \sqrt{\frac{\bar{\theta}_{\max }}{\lambda_{\min }(\mathbf{P}) \Gamma}}
$$

where $\lambda_{\min }(\mathbf{P})$ is the minimum eigenvalue of $\mathbf{P}$, and $\bar{\theta}_{\text {max }}$ is defined by the following expression.

$$
\bar{\theta}_{\max }=\max _{\theta \in \Theta} \sum_{i=1}^{n} 4 \theta_{i}^{2}
$$

\subsection{3 $\mathcal{L}_{1}$ Control Architecture}

In its current form, the CMAC control algorithm is an acceptable controller for systems with uncertain input gain, however, Hovakimyan and Cao [59] demonstrate that as one increases the adaptation rate $\Gamma$, the tracking performance is improved at the expense of robustness. This can be explained upon further inspection of Eqs. (4.39) to (4.41); where one can conclude that by increasing the magnitude of the adaptation rate $\Gamma$, the adaptive parameters become increasingly sensitive to changes in the error signal $\tilde{\mathbf{x}}(t)$. As a result, the rate of change of the control signal $\mathbf{u}(t)$ could exceed the capabilities of the plant, and therefore may drive the closed-loop system to instability.

The $\mathcal{L}_{1}$ control algorithm is a relatively simple extension of the CMAC architecture that addresses this issue by applying a filtering technique which allows the performance bounds to be decoupled from the robustness of the closed-loop system. This means that the controlled system can achieve both relative fast adaptation and robustness simultaneously. 
To apply this technique, let us first take the Laplace transforms of $\hat{\boldsymbol{\eta}}(t)$ to be $\hat{\boldsymbol{\eta}}(s)$, and the reference signal $\mathbf{r}(t)$ to be $\mathbf{R}(s)$. By applying a strictly proper transfer function (or low-pass filter) $D(s)$ to Eq. (4.43), the $\mathcal{L}_{1}$ control law is defined by Eq. (4.46) below.

$$
\mathbf{U}(s)=-k D(s)\left(\hat{\boldsymbol{\eta}}(s)-k_{g} \mathbf{R}(s)\right)
$$

where $k$ is a positive scalar selected by the designer. Figure 4.4 shows the full closedloop system of the $\mathcal{L}_{1}$ control scheme.

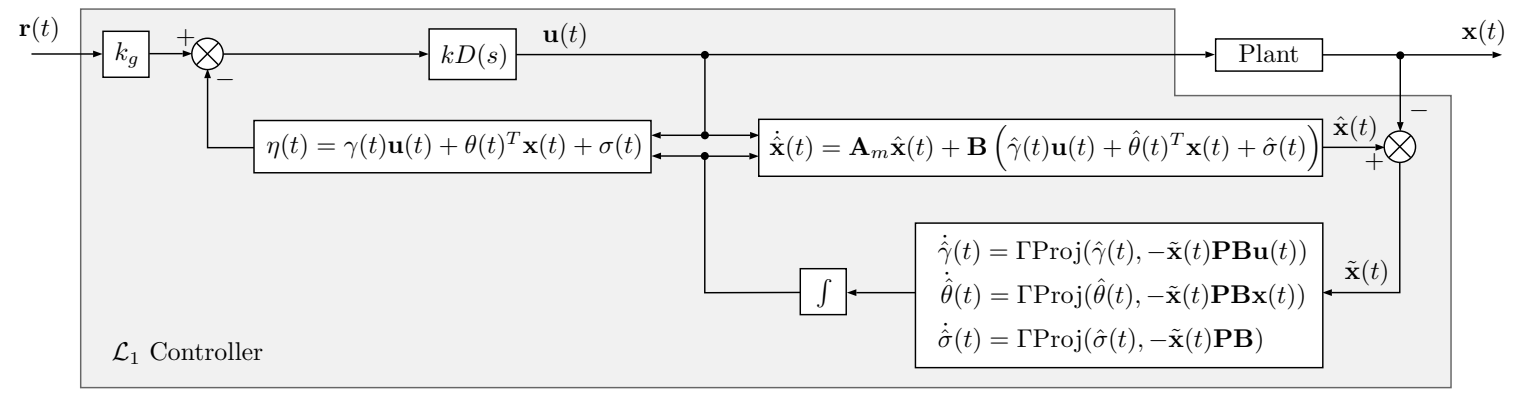

Figure 4.4: $\mathcal{L}_{1}$ control architecture for systems with uncertain input gain.

Despite the simplicity of adding a low-pass filter to the control signal, there are criteria that must be met before it can be deemed " $\mathcal{L}_{1}$ " stable (thus earning its namesake as an $\mathcal{L}_{1}$ controller). Let us first consider the closed-loop form of the filter $D(s)$, which must yield a strictly proper, bounded-input bounded-output (BIBO) stable transfer function $C(s)$ that has a finite gain as $s \rightarrow 0$.

$$
C(s)=\frac{k \gamma D(s)}{1+k \gamma D(s)} \quad \forall \quad \gamma \in \Upsilon
$$

In order for the system to be considered $\mathcal{L}_{1}$ stable, $C(s)$ and $\gamma$ must satisfy the following condition.

$$
\|G(s)\|_{\mathcal{L}_{1}} L<1
$$

where: 


$$
\begin{gathered}
L \triangleq \max _{\theta \in \Theta}\|\boldsymbol{\theta}(t)\|_{1} \\
H(s) \triangleq\left(s \mathbb{I}-\mathbf{A}_{m}\right)^{-1} \mathbf{B} \\
G(s) \triangleq H(s)(1-C(s))
\end{gathered}
$$

Now, let us consider a closed-loop reference system for the class of system outlined previously in Eq. (4.37) for the non adaptive version of this controller.

$$
\begin{gathered}
\dot{\mathbf{x}}_{r e f}(t)=\mathbf{A}_{m} \mathbf{x}_{r e f}(t)+\mathbf{B}\left(\gamma(t) \mathbf{u}_{r e f}(t)+\boldsymbol{\theta}(t)^{T} \mathbf{x}_{r e f}(t)+\boldsymbol{\sigma}(t)\right) \\
\mathbf{U}_{r e f}(s)=-\frac{C(s)}{\gamma}\left(\boldsymbol{\eta}_{r e f}(s)-k_{g} \mathbf{R}(s)\right)
\end{gathered}
$$

where $\mathbf{x}_{r e f}, \mathbf{U}_{r e f}(s)$, and $\boldsymbol{\eta}_{r e f}(s)$ correspond to the closed-loop signals of $\mathbf{x}, \mathbf{U}(s)$, and $\boldsymbol{\eta}(s)$, respectively.

Theorem 2. If the selection of $k$ and $D(s)$ verify $\|G(s)\|_{\mathcal{L}_{1}} L<1$, then the closedloop reference system in Eqs. (4.52) to (4.53) is bounded-input bounded-output stable with respect to $\mathbf{r}(t)$ and the initial condition $x_{0}$.

Proof. For the sake of brevity, please refer to the proof outlined by Zou et al. in [58], or by Hovakimyan and Cao in [59].

This implies that the stability of the system greatly depends on the user's selection of the filter $D(s)$, the companion system matrices $\mathbf{A}_{m}$ and $\mathbf{B}$, and the boundaries on the parameters $\gamma(t)$, and $\boldsymbol{\theta}(t)$. As such, these parameters cannot be arbitrarily selected and careful consideration must be exercised when designing the $\mathcal{L}_{1}$ controller. 


\section{Chapter 5}

\section{Controller Simulations and Tuning}

Before the control algorithms are implemented into the experimental testbed software, it was deemed appropriate to create simulations of the plant and controllers for a number of reasons. The primary purpose is to develop the control laws in a simulated environment to ensure that the algorithms are functioning as expected prior to experiments. This will allow for rapid troubleshooting and will eliminate the risk of damaging the experimental apparatus during the coding process. Furthermore, since MATLAB/Simulink is used to run the experimental hardware, the control laws developed in simulation can be seamlessly implemented into the testbed software with only minor modifications required.

In addition to verifying controller functionality, another key purpose of these simulations is to provide a virtual tool to understand how to tune the adaptive control laws in a general sense for a system of similar likeness. In doing so, the effects of certain parameters on system response could be observed in a controlled environment, without creating any unnecessary risk to the experimental testbed. Moreover, by simulating a plant that is similar to what is actually implemented in experiment, the gains found in simulation will act as a suitable starting point for the tuning process of the experiment.

Thus, this chapter will cover the simulation and parameter identification of the plant in Section 5.1, the implementation of the baseline PID controller in Section 5.2, and the implementation of the $\mathrm{AB}$ and $\mathcal{L}_{1}$ adaptive controllers in Sections 5.3 and 5.4 respectively.

\subsection{Simulating the Plant}

To simulate the plant, there are two main components that must be considered: the CSTM and the MX-28 servomotors. To ensure that the simulated plant is similar 
to the actual plant, the parameters and models associated with these two components were extracted using the Parameter Estimation Toolbox within Simulink from experimental data. Thus, Section 5.1.1 will cover the set-up of the Simulink environment, Section 5.1.2 will cover the model identification of the actuators, and finally Section 5.1.3 will cover the parameter estimation of the model parameters for the actual CSTM from experimental data.

Note that since the simulations are being used solely as a tool for preliminary analyses, it was deemed unnecessary to verify the accuracy of the plant model parameters against other manoeuvres in experiment. As a result, the simulated performance of the controllers cannot be directly compared to the experimental results; rather, the general performance characteristics between simulations and experiments will be compared in a qualitative manner in Chapter 6.

\subsubsection{Setting Up the Simulated Environment}

For the sake of simplicity, the simulations were designed to simulate a single link at any given time. For the purposes of this section, only the the LP joint is considered. The top-level Simulink diagram was structured as per Fig. 5.1 below, and runs on a discrete time solver to better match the experiment. For the sake of modularity, the plant consists of two separate systems containing the actuator and CSTM models as independent modules, and the controller block contains all necessary logic for the desired controller (i.e. PID, AB, or $\mathcal{L}_{1}$ ).

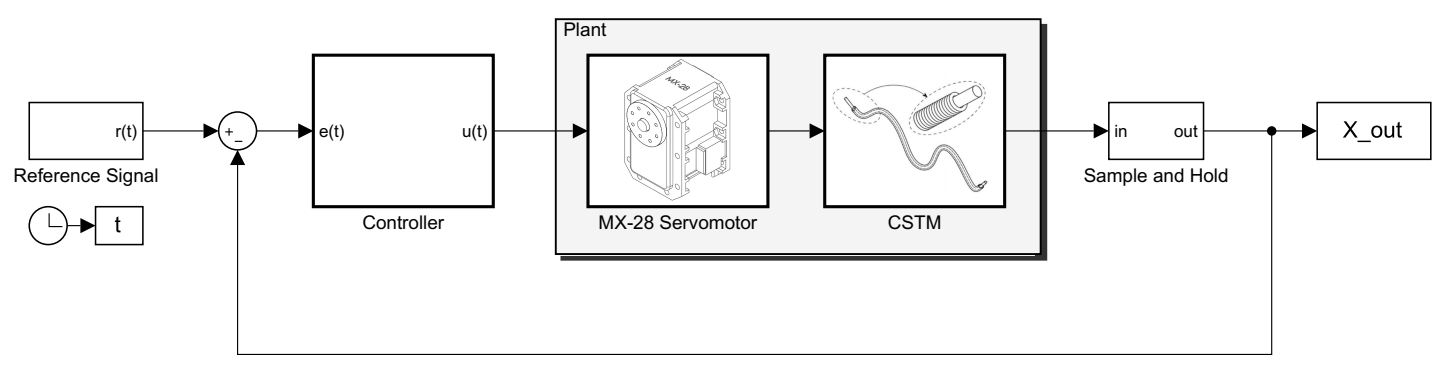

Figure 5.1: General control architecture for systems with uncertain input gain.

The sample and hold block within this diagram is designed to simulate the sampling nature of the PhaseSpace measurement system used within the experiments. 
As mentioned in Section 3.3, the Simulink diagram must be run at a higher rate than the sample rate of the LED position measurements. To replicate this in simulation, the output produced by the plant module is sampled and held at a lower rate. In this case, the plant model is propagating with the Simulink diagram at $100 \mathrm{~Hz}$, however, the output of the plant is only recorded at a rate of $50 \mathrm{~Hz}$; which is the rate of the PhaseSpace server measurements.

\subsubsection{Actuator Model Identification}

With the general software architecture created, the components of the plant can now be modelled. Normally, stand-alone servomotors can be modelled as DC motors with a gearbox and a simple internal PID controller [60]. Unfortunately, the servomotors in the experiment are coupled with hysteretic friction, a torsional stiffness at the gripper joints, and an additional inertia associated with the gripper links. A simplified schematic of an analogous system can be seen in Fig. 5.2.
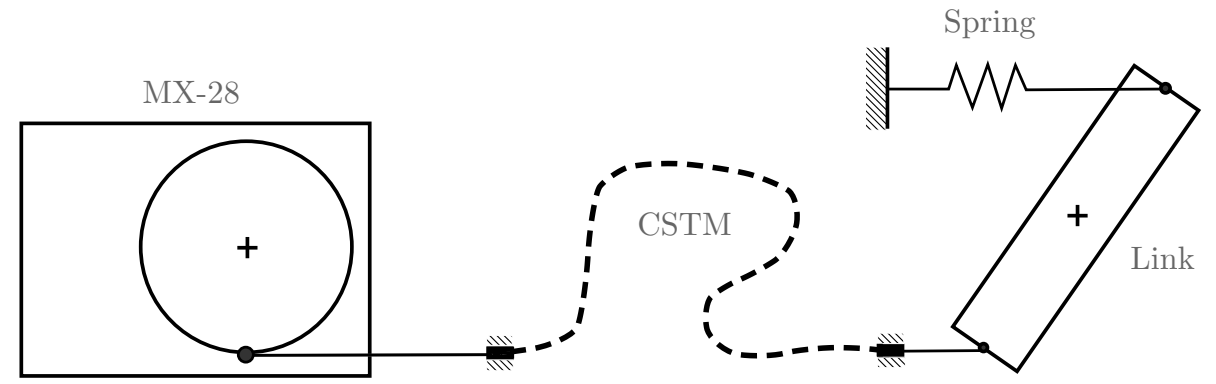

Figure 5.2: Simplified schematic of the actuation system in the testbed.

Since the dynamics of the gripper are coupled to the actuator, their effect on the servomotor cannot be neglected. Luckily, a high fidelity model is not required for this simulation since the objective is not to match the experiment perfectly; rather to have a similar performance. Thus, it was deemed appropriate to treat the system as a black-box system with an unknown strictly proper transfer function. This can be accomplished by making use of the System Identification Toolbox in MATLAB by providing experimental data of the system for a given input signal.

To do so, the MX-28 actuators in the fully assembled gripper were sent a multistep input with an increasing amplitude of $10 \%$ of the full range of the actuator. To 
ensure that the effects of hysteresis were not captured in the data, the encoder values of the actuators were sampled directly. The script used to initialize the connection, command the actuators, and record the encoder data was programmed in MATLAB using the Dynamixel Protocol 2.0 library (refer Appendix B.3 for the script). The response of the actuation system for the LP link is given by Fig. 5.3, where the vertical axis represents the servomotor output in bits as a function of time. These results were recorded at a rate of $60 \mathrm{~Hz}$ via USB connection. Upon initial inspection of the response, it is noted that as the magnitude of the step command increases, the response generally demonstrates a greater steady state error. This is a reasonable outcome since the torque generated by the spring is a function of angular displacement, and as the joint angle increases, the servomotor must overcome a greater tension in the cable.

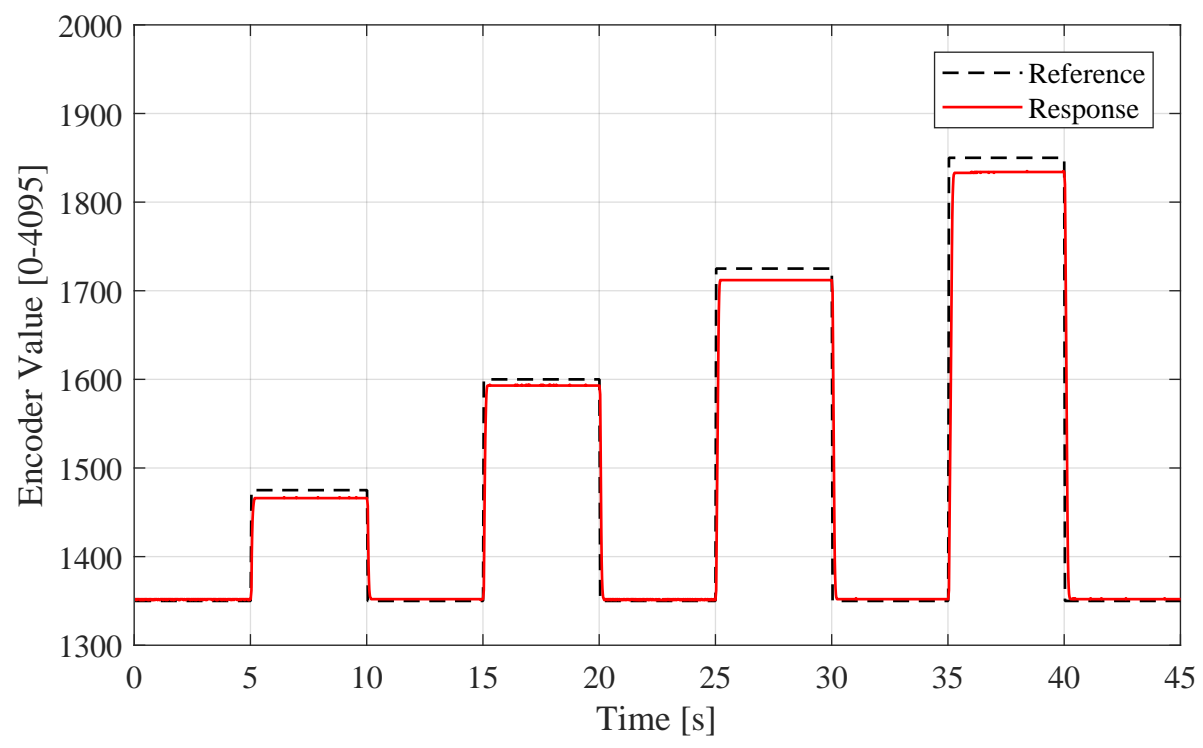

Figure 5.3: Multi-step response of the actuation system controlling the LP joint.

By importing this data into the System Identification Toolbox, the model estimation algorithm will attempt to minimize the mean-squared-error (MSE) of the experimental data and the estimated model for the same input. Since this is an optimization routine, the solver and its terminating conditions can be adjusted to yield the best results. For the purposes of this analysis, MATLAB's internal fmincon algorithm was selected and set to run based on SQP method (as used in Chapter 2 
for details) with the maximum iterations set to 1000 .

Furthermore, the user must define the type of model and its form prior to running the optimization routine. It is assumed that the full actuation system is a black-box strictly proper transfer function of unknown order. To determine the numerator and denominator order of the transfer function, a systematic trial-by-error approach was taken to determine the case with the most suitable fit. Of the many tests that were completed, the two cases with the best $R^{2}$ value include: Case 1) a 2 zero -3 pole transfer function with an $R^{2}=95.26$, and Case 2) a 2 zero - 5 pole transfer function with an $R^{2}=95.84$. The coefficients of these transfer functions are presented in Table 5.1, which are used by the generalized continuous transfer function defined in Eq. (5.1).

$$
A(s)=\frac{b_{2} s^{2}+b_{1} s+b_{0}}{a_{5} s^{5}+a_{4} s^{4}+a_{3} s^{3}+a_{2} s^{2}+a_{1} s+a_{0}}
$$

Table 5.1: Transfer function coefficients for Case 1 and Case 2.

\begin{tabular}{|c|c|c|}
\cline { 2 - 3 } \multicolumn{1}{c|}{} & Case 1 & Case 2 \\
\hline \hline$a_{0}$ & 5.734 & $1.521 \times 10^{4}$ \\
\hline$a_{1}$ & $2.835 \times 10^{2}$ & $1.085 \times 10^{3}$ \\
\hline$a_{2}$ & 33.81 & $6.779 \times 10^{4}$ \\
\hline$a_{3}$ & 1.000 & $4.836 \times 10^{3}$ \\
\hline$a_{4}$ & 0 & 96.53 \\
\hline$a_{5}$ & 0 & 1.000 \\
\hline$b_{0}$ & 5.671 & $1.516 \times 10^{4}$ \\
\hline$b_{1}$ & $2.800 \times 10^{2}$ & $-2.824 \times 10^{2}$ \\
\hline$b_{2}$ & 13.98 & $6.653 \times 10^{4}$ \\
\hline
\end{tabular}

Based solely on the $R^{2}$ value, one would assume that Case 2 would be the better choice; however, upon closer inspection of the transfer function response in comparison to the measurements shows that Case 1 demonstrates better steady state characteristics (i.e. no transient behaviour at the peaks of the step). This is evident 
by the simulated multi-step response of each transfer function given in Fig. 5.4, with the error between each model and the measurements given in Fig. 5.5.

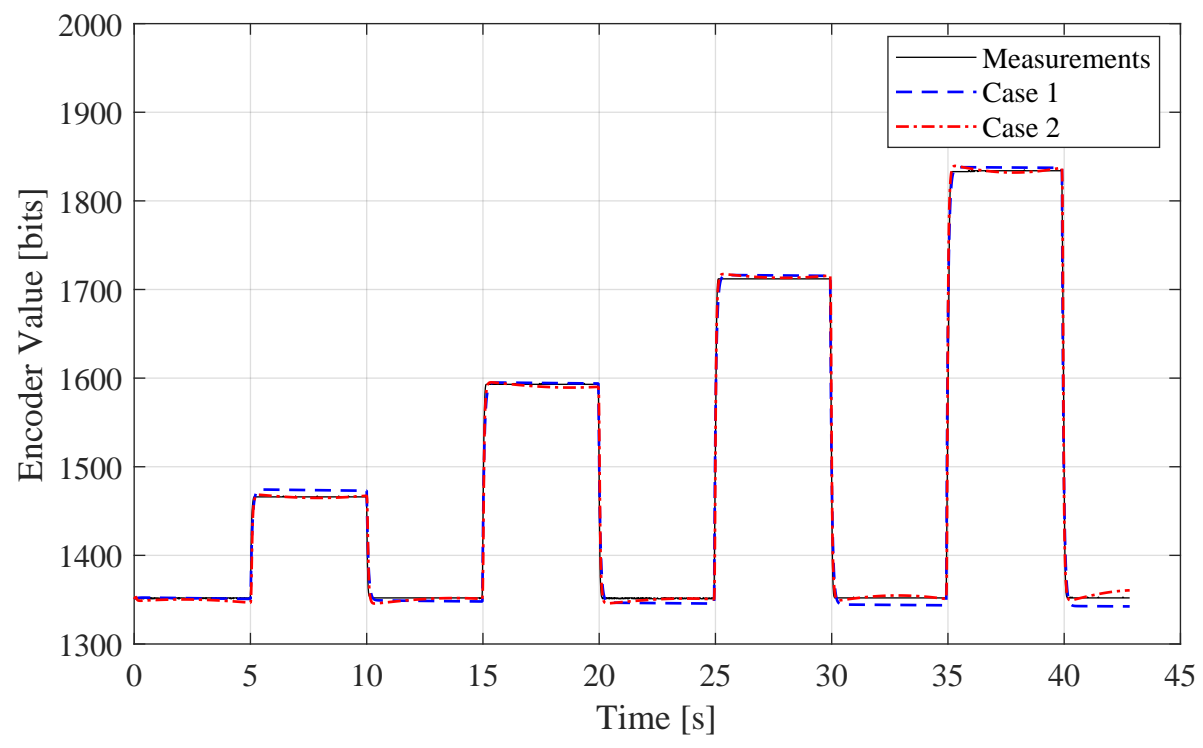

Figure 5.4: Multi-step response of the Case 1 and Case 2 compared to experimental data.

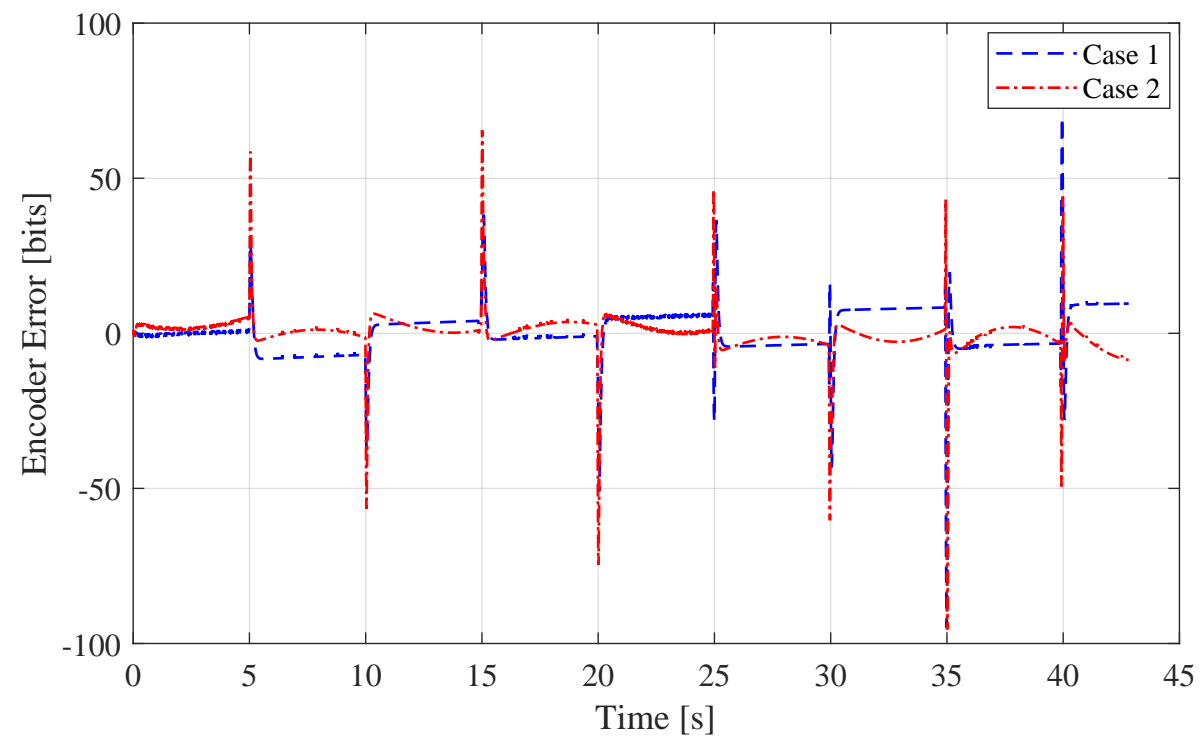

Figure 5.5: Absolute error of Case 1 and Case 2 with respect to experimental data.

Since Case 1 demonstrates a response that is more similar in shape to what was 
observed experimentally, it will be selected as the motor model in the simulation. It is recognized that there are obvious errors between the selected model and the experimental apparatus, however, it is sufficient given the purpose of the simulations.

Within the "MX-28" block in Fig. 5.1, the model is implemented as a discretetime state-space representation of the transfer function (see Fig. 5.6). The conversion from continuous transfer function to discrete state-space is shown in lines 35 to 43 of the RUN_none_LP_sim.m script in Appendix B.5.

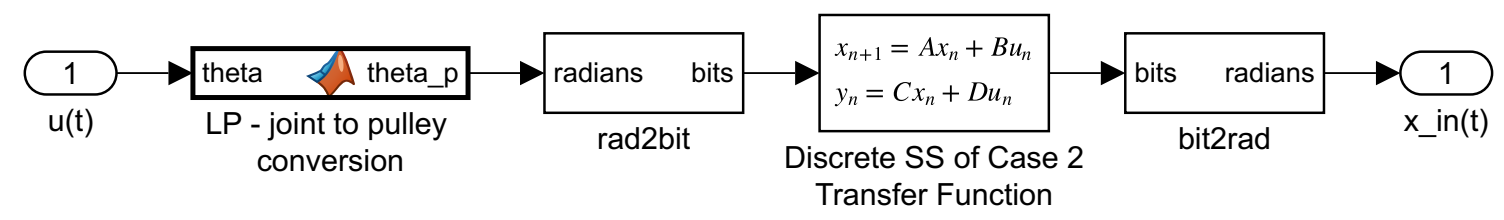

Figure 5.6: Implementation of Case 2 model into Simulink.

Since the model developed was calibrated using encoder bit values, a conversion was included to ensure unit consistency. Additionally, the conversion which translates the desired joint angle into actuator angle (as per Eq. (3.14)) was included as it is in the experimental set-up.

\subsubsection{CSTM Modelling and Parameter Estimation}

The CSTM can be simulated as per the modified Bouc-Wen model outlined in Section 4.2.1, where its parameters can be extracted from experimental data of the uncontrolled system. Figure 5.7 shows the Simulink implementation of the CSTM model, where the user defined function blocks "alpha_x", "d_zeta", and "phi" are the functions from Eqs. (4.3), (4.4) and (4.2) respectively. Blocks [A]-[E] correspond to the constants $\alpha_{1}, \alpha_{2}, A, \nu$, and $\delta$ respectively, which are the unknown model parameters of the CSTM to be determined by the Parameter Estimation Toolbox within MATLAB. 


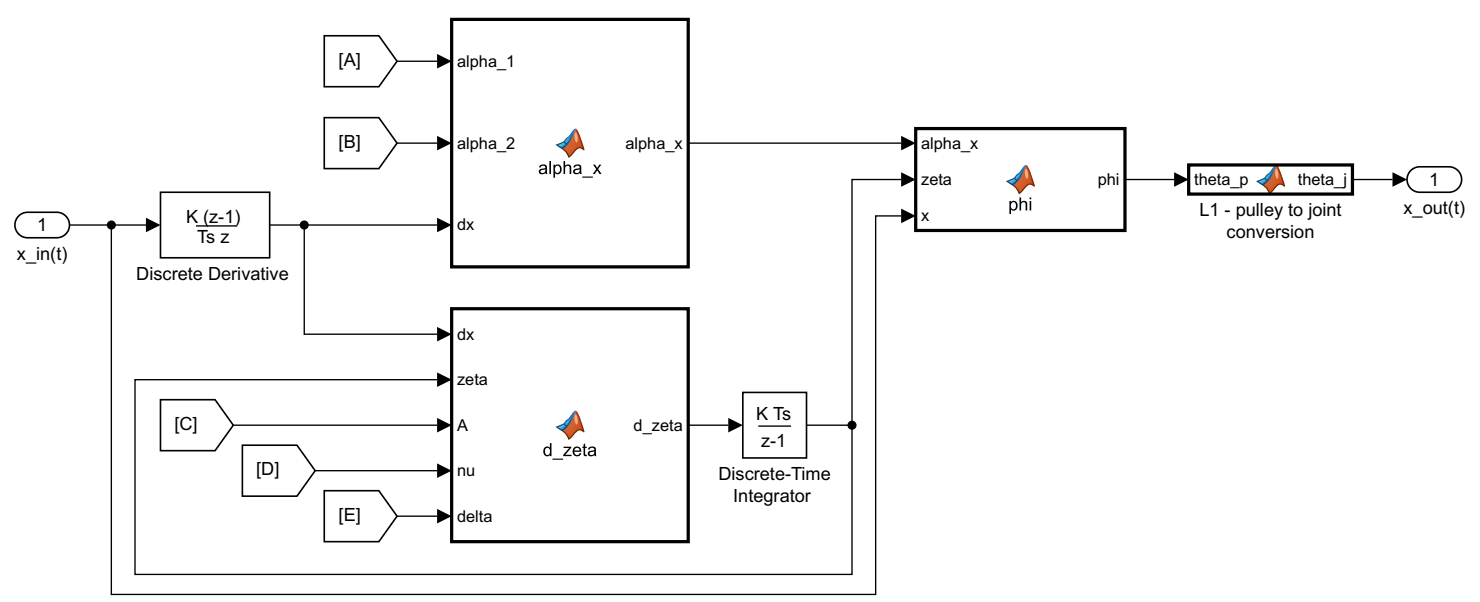

Figure 5.7: Implementation of the CSTM model into Simulink.

To run the estimation tool, the uncontrolled response of the system must be acquired experimentally. The LP link of the gripper was fed the "Manoeuvre 1" reference signal (see Section 6.1 for details) directly to the actuators, and the joint angle was measured over the duration of the manoeuvre, as shown by Fig. 5.8.

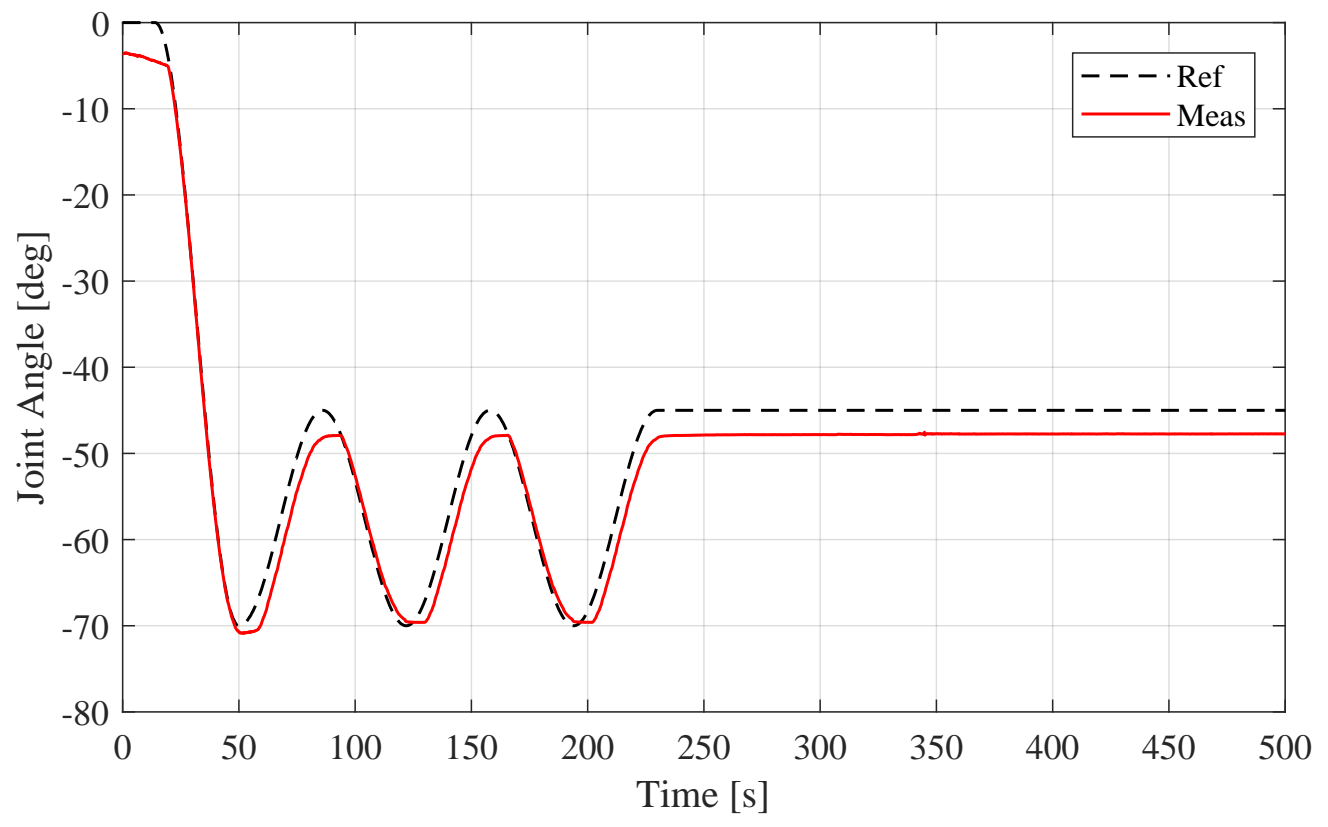

Figure 5.8: Experimental data of the LP joint of Manoeuvre 1. 
This data was compared against the simulated plant where a MSE minimization routine is run between the two. The simulated plant used in the parameter estimation process is the MX-28 servomotor and the CSTM, as highlighted in Fig. 5.1. To reduce the number of iterations in the optimization routine, the initial estimate of the CSTM parameters were taken from a similar system by Do et al. [44] with values being $\alpha_{1}=0.8972, \alpha_{2}=0.9487, A=-0.8745, \nu=5.034$, and $\delta=0.1452$. With these parameters set, the estimation routine took 8 iterations to converge to a final MSE of $1.880 \times 10^{-4}$. Figure 5.9 shows the propagation of the estimated parameters throughout each iteration, where the final values were found to be $\alpha_{1}=1.677, \alpha_{2}=$ 0.2968, $A=-0.3572, \nu=16.97$, and $\delta=1.094 \times 10^{-6}$. The performance of these estimated parameters with respect to the experimental data is given in the following section.

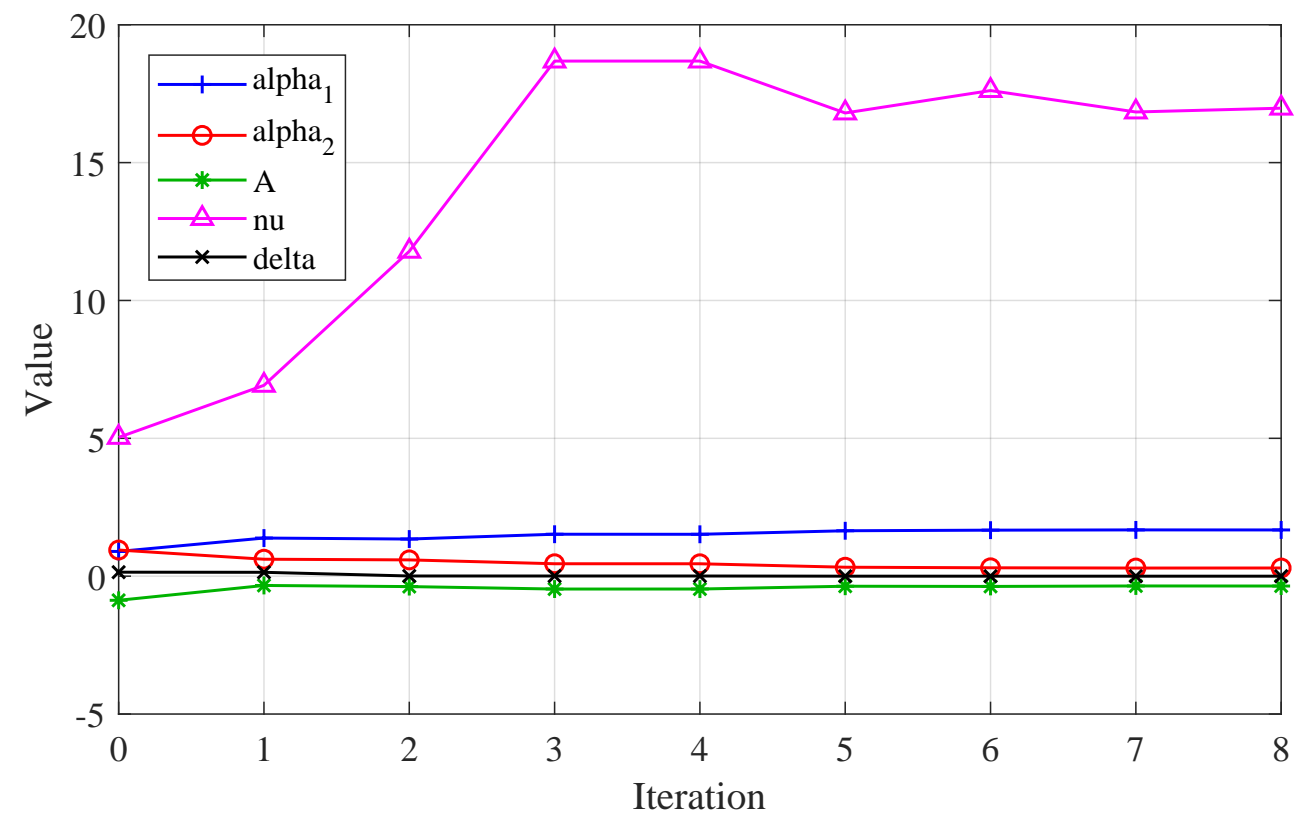

Figure 5.9: Propagation of CSTM parameters within the parameter estimation routine.

\subsubsection{Full Plant Simulation}

With the actuation system and the CSTM modelled, the response of the fully simulated plant can be compared to experimental data. For the sake of consistency, the 
full plant was given the Manoeuvre 1 reference signal so that it could be compared directly to the experimental data captured in Fig. 5.8. The simulation results demonstrate a high degree of likeness with respect to the experimental data, where the coefficient of determination was found to be $R^{2}=99.59 \%$. This is further reinforced by Fig. 5.10, where the experimental data and the simulation data of the LP joint angle as a function of time are shown for the same manoeuvre.

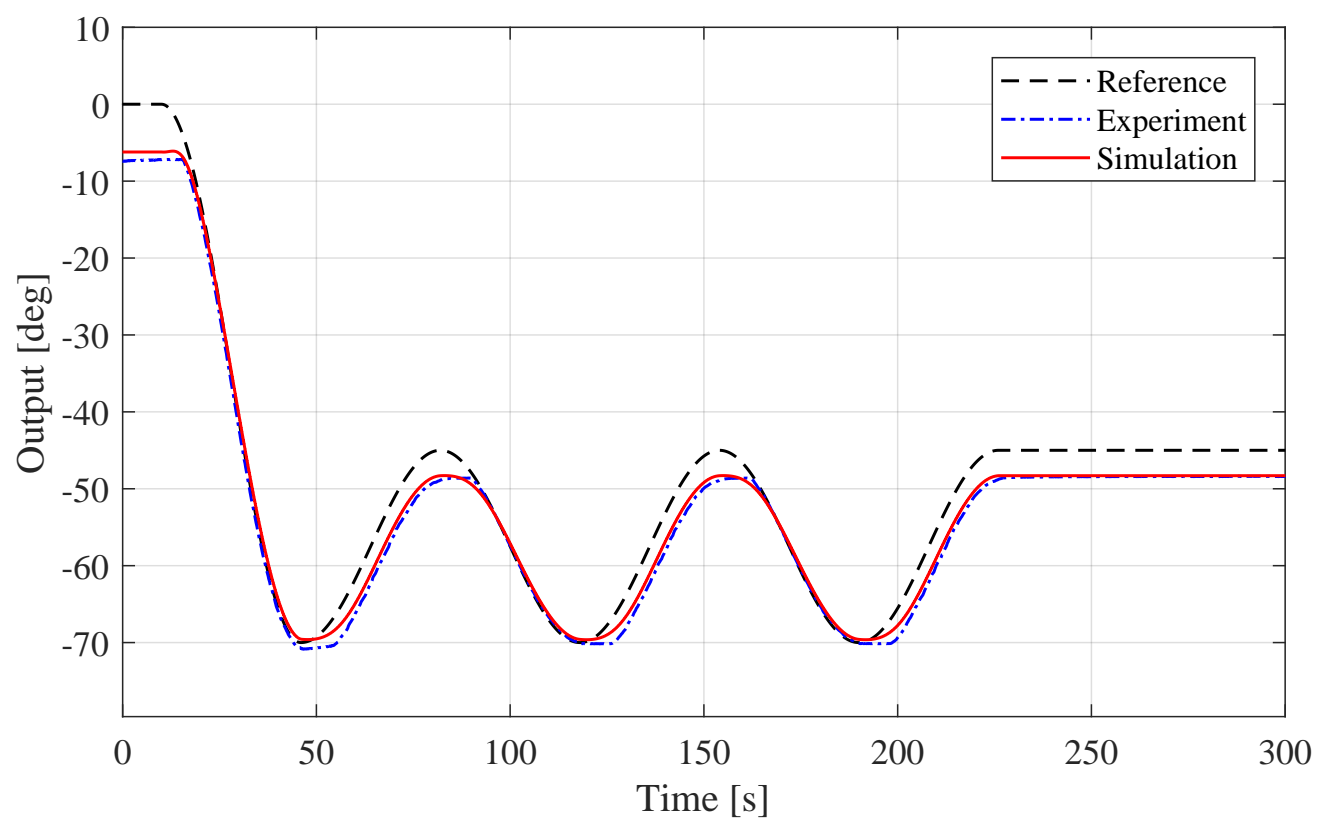

Figure 5.10: Comparison of experimental and simulated data of the LP joint.

It can therefore be stated that the simulated plant will serve as a reasonable approximation of the experimental hardware for Manoeuvre 1. However, it is noted that the CSTM parameters were estimated using only a single manoeuvre. It would be best practice to run multiple manoeuvres in experiment and then validate the model parameters using that data, however, the purpose of the simulations is to simply emulate a similar system for controller programming purposes. Therefore, to maintain continuity and to ensure that the fidelity of the simulated plant is consistent, the simulations will only be run using Manoeuvre 1.

For the purposes of later comparison, the tracking performance, tracking error of the simulated plant, and the hysteresis of the transient phase is given by Fig. 5.11. 

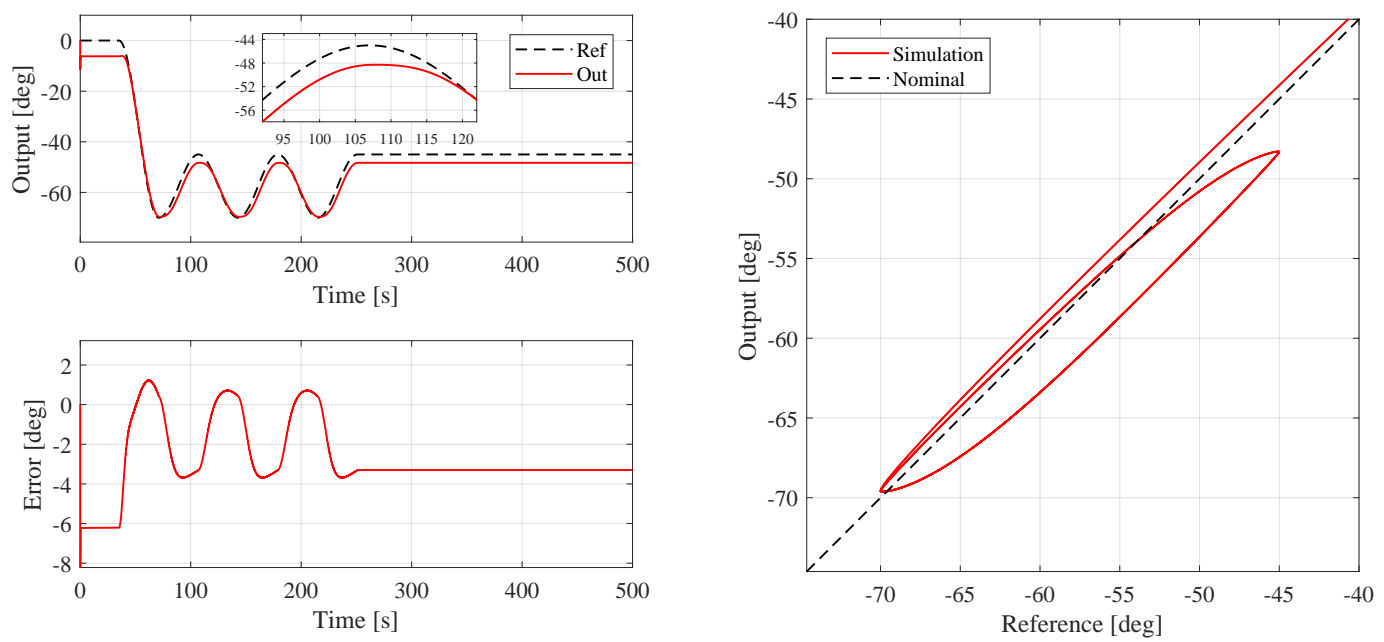

Figure 5.11: Simulated LP joint performance under no control. Top: actual and desired proximal link angular trajectory, bottom: trajectory tracking error, and right: hysteresis loop of transient phase.

Based on these plots, the model appears to have a large steady state error and a large phase delay in the response; which is identifiable by the width of the hysteresis loop. Note that for perfect set point tracking, the hysteresis loop would lie perfectly on the "nominal" line, and meets the limits of the manoeuvre (in this case, $-45 \mathrm{deg}$, and $-70 \mathrm{deg}$ ). It is clear that the uncontrolled system does not perform adequately, which is to be expected given the nature of the system.

\subsection{Implementation of PID}

As it was mentioned in Section 4.1, the PID controller will be used as a baseline case when analysing the controlled response of the system. Due to its simplicity, the PID algorithm was implemented in parallel form (see Fig. 5.12), where $K_{p}, K_{i}$, and $K_{d}$ correspond to the proportional, integral, and derivative terms respectively. 


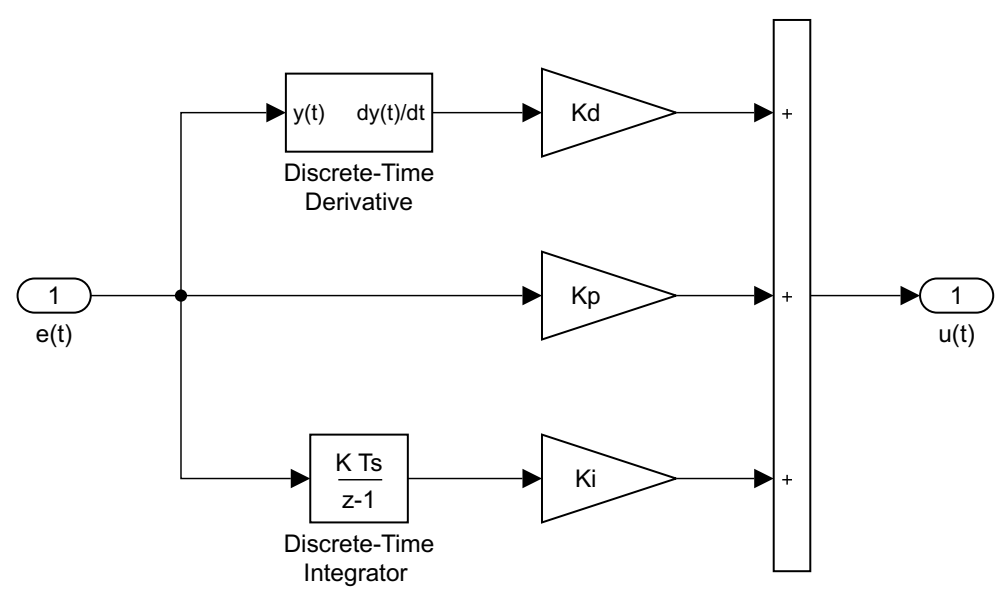

Figure 5.12: Simulink diagram of the implemented PID controller.

When this controller is implemented into the controller block of the simulation as per Fig. 5.1, the closed-loop system response is notably improved. With gains of $K_{p}=0.850, K_{i}=1.21$, and $K_{d}=0.113$, the system performance is reported by Fig. 5.13. These gains were selected to give the simulation the most desirable performance through the process of trial and error to reduce overshoot and phase delay. Note that the controller is not enabled until $t=35$ seconds to maintain continuity with the experiments in Chapter 6 .
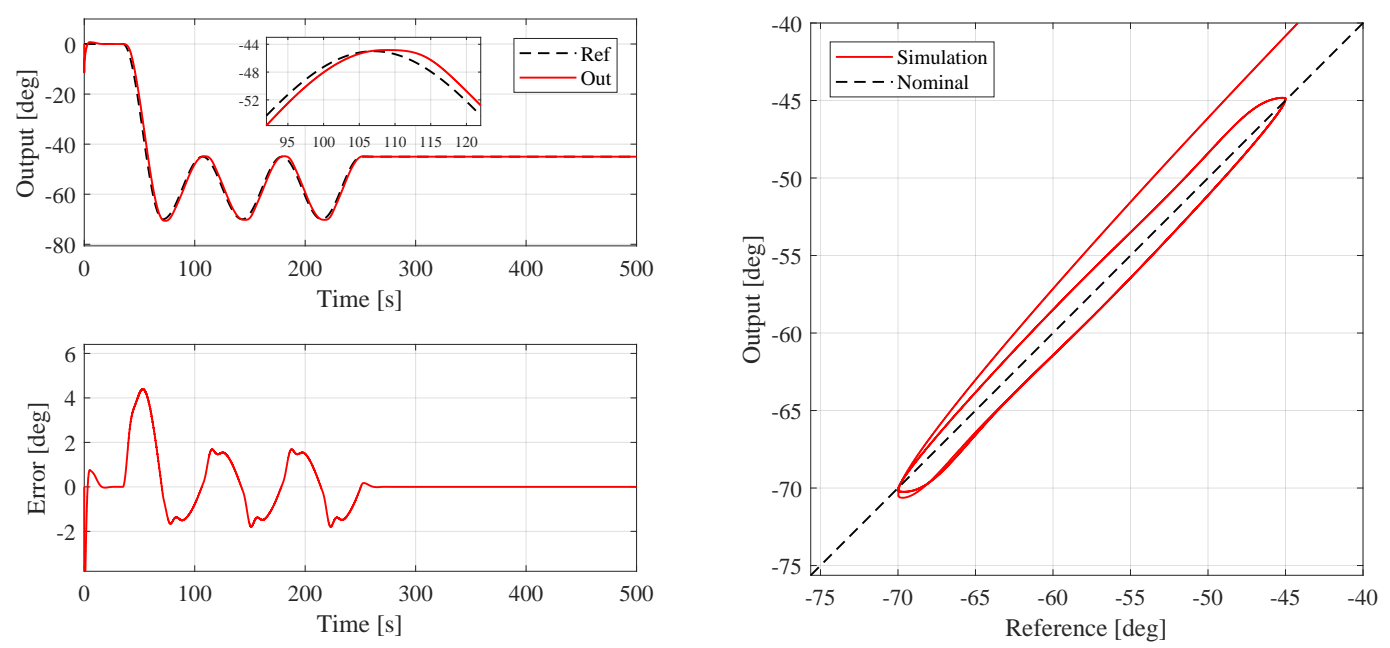

Figure 5.13: Simulated LP joint performance under PID control. Top: actual and desired proximal link angular trajectory, bottom: trajectory tracking error, and right: hysteresis loop of transient phase. 
Since it is so thoroughly researched, the details of how each gain affects the system response will not be covered in this thesis. As a general comment on the PID controller performance, the closed-loop system was indeed able to converge the steady state error to zero, however, there were no combinations of gains found that could offset the phase delay in the response.

\subsection{Implementation of AB Control}

To implement the $\mathrm{AB}$ controller, the same general structure for the system diagram outlined in Fig. 5.21 is used devoid of the state vector conversion block, since the angular rate is not required for the algorithm. Within the controller block, the AB algorithm is executed as per Fig. 5.14, where the "omega(t) and d_er(t)" block determines $\dot{e}_{r}(t)$ and evaluates Eq. (4.8) to find $\omega(t)$, the "dDelta(d)/dt" block determines $\dot{\hat{\Delta}}_{n}^{*}(t)$ as per Eq. (4.25), the "d_chi" blocks execute Eqs. (4.23) and (4.24) for $\dot{\hat{\chi}}_{1}(t)$ and $\dot{\hat{\chi}}_{2}(t)$, respectively, the "ubar" blocks compute the virtual controllers $\bar{u}_{1}(t)$ and $\bar{u}_{2}(t)$ from Eqs. (4.20) and (4.21), and finally "Control Law" block finds the control input $u(t)$ by Eq. (4.22).

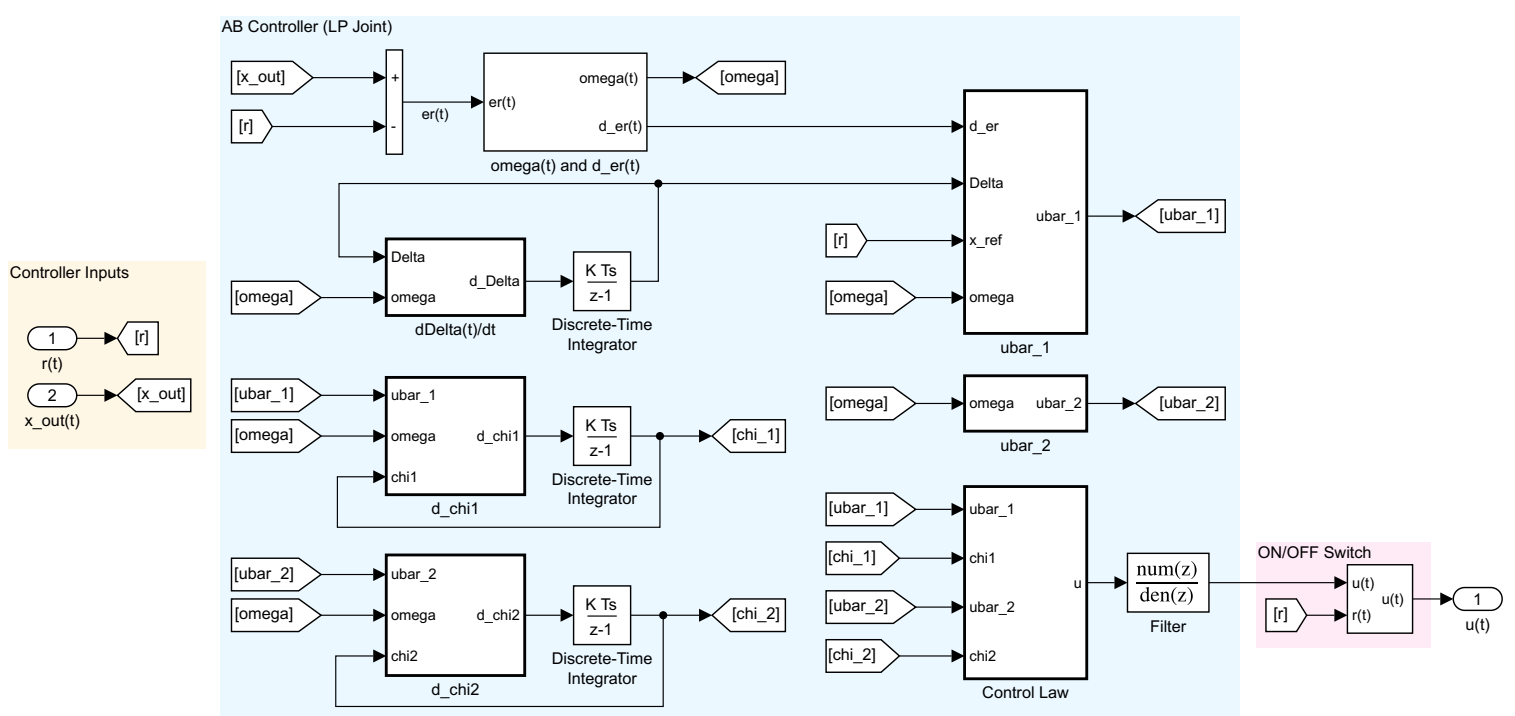

Figure 5.14: Algorithm implementation for the $\mathrm{AB}$ controller for the LP joint. 
Note that in this implementation, there is the same control switch from the $\mathcal{L}_{1}$ simulation diagram, and the addition of a filter at the control signal. This filter, though not mentioned in the literature, was necessary to run the simulations since the adaptation laws produce high frequency oscillations due to rate inversions (a mathematical phenomenon) which cannot be evaluated numerically by the plant model. For the case of the simulation, the filter was selected based on trial and error with the objective of damping out the high frequency oscillations, and found to be a discrete first-order low-pass filter below, which remained fixed throughout the tuning process.

$$
f(z)=\frac{3.333 \times 10^{-4}}{z-0.9997}
$$

\subsubsection{Sensitivity Analysis on AB Controller Parameters}

The tuning process for the $\mathrm{AB}$ controller involves properly selecting the values for ten

parameters, including $k_{1}, k_{2}, \delta_{1}, \delta_{2}, \delta_{3}, \sigma_{1}, \sigma_{2}, \sigma_{3}, \epsilon$ and $\alpha$. Fortunately, as was done previously for the $\mathcal{L}_{1}$ controller tuning analysis, there are a few assumptions that can be made to reduce the number of tunable parameters. The first assumption is that the value of $\epsilon$ contributes a negligible effect on the controller performance since it is simply a smoothing parameter for the hyperbolic tangents within the control law. This assumption was reinforced through a simulation, where a large variation in $\epsilon$ produced no noticeable effect on the closed-loop response. As such, this parameter was set to be $\epsilon=0.05$, which was the value selected in Do's work $[45,46]$.

Another assumption that can be made is that the parameters associated with the adaptation laws are all the same magnitude such that $\delta_{1}=\delta_{2}=\delta_{3}=\delta$, and $\sigma_{1}=\sigma_{2}=\sigma_{3}=\sigma$. This strategy was also used by Do [45], which demonstrated that excellent control performance was still achievable under this assumption. Thus, there are now only five parameters to tune $\left(k_{1}, k_{2}, \delta, \sigma\right.$, and $\left.\alpha\right)$, which greatly simplifies the tuning process.

As before, the control parameters were initially selected arbitrarily, and manipulated individually to determine their isolated effects on the closed-loop response of the LP joint under Manoeuvre 1 in the transient-hold transition period. The initial values for these parameters were selected to be $k_{1}=k_{2}=\alpha=1$ and $\delta=\sigma=1$. 
Figure 5.15 to 5.19 shows the simulated response of the LP joint angle as each parameter is varied, where the black-asterisk marked curve represents the common response between all figures.

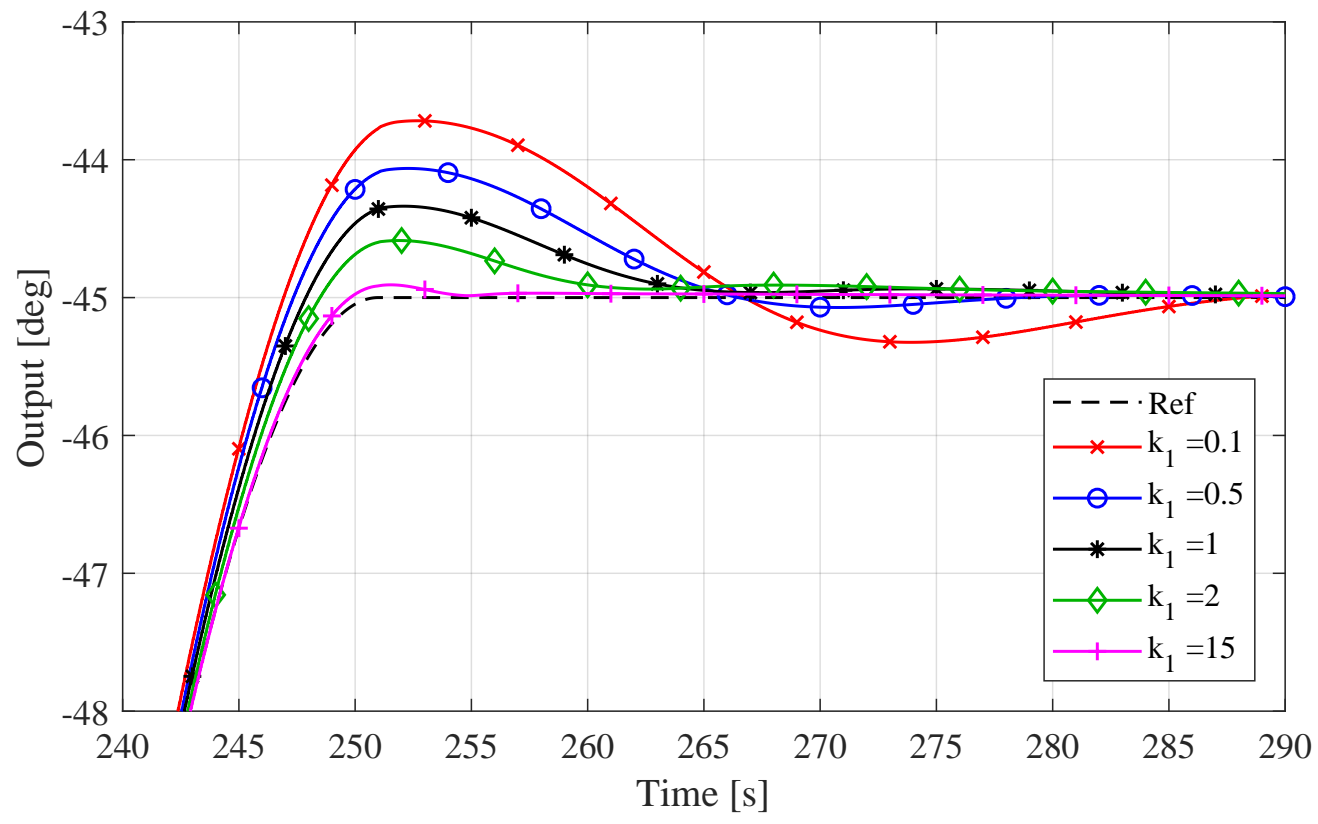

Figure 5.15: Effect of $k_{1}$ on closed-loop response. 


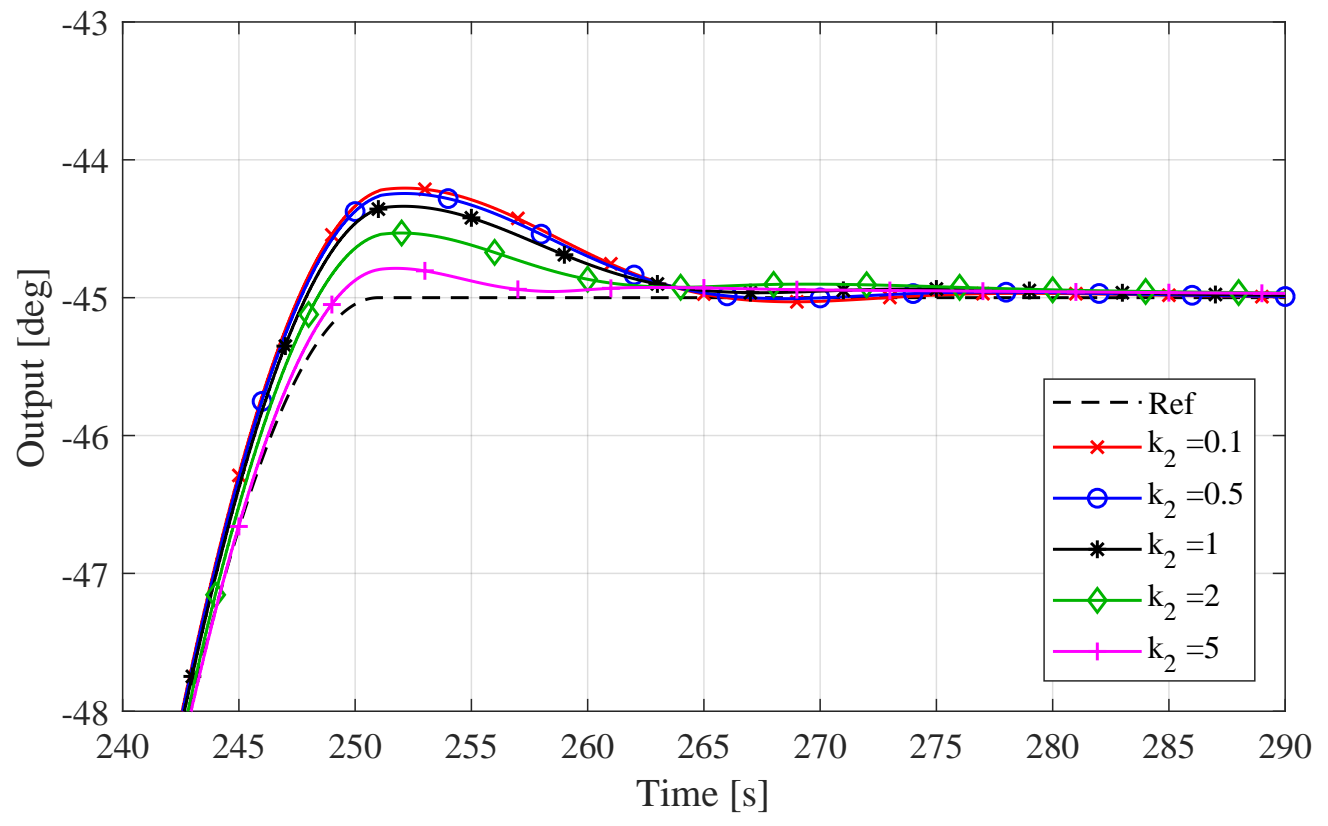

Figure 5.16: Effect of $k_{2}$ on closed-loop response.

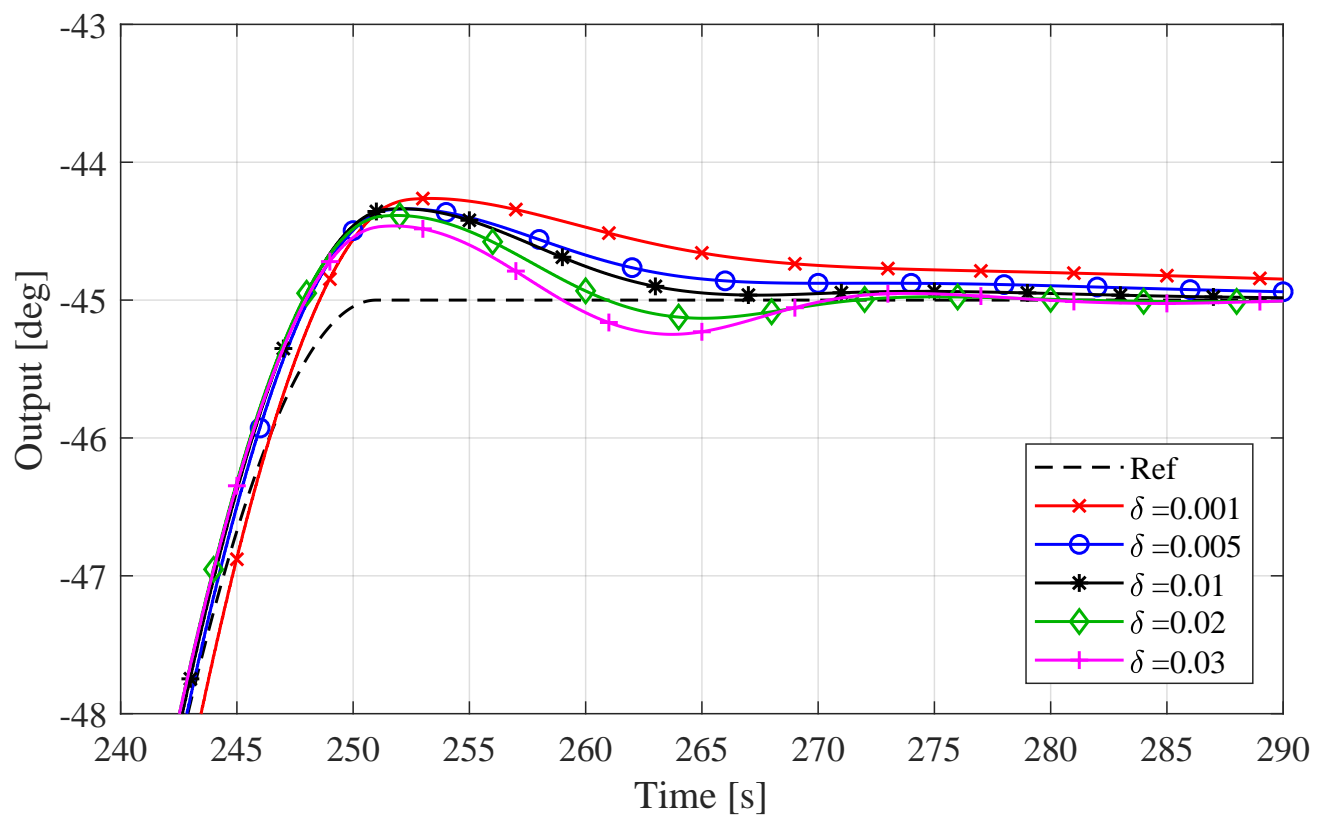

Figure 5.17: Effect of $\delta$ on closed-loop response. 


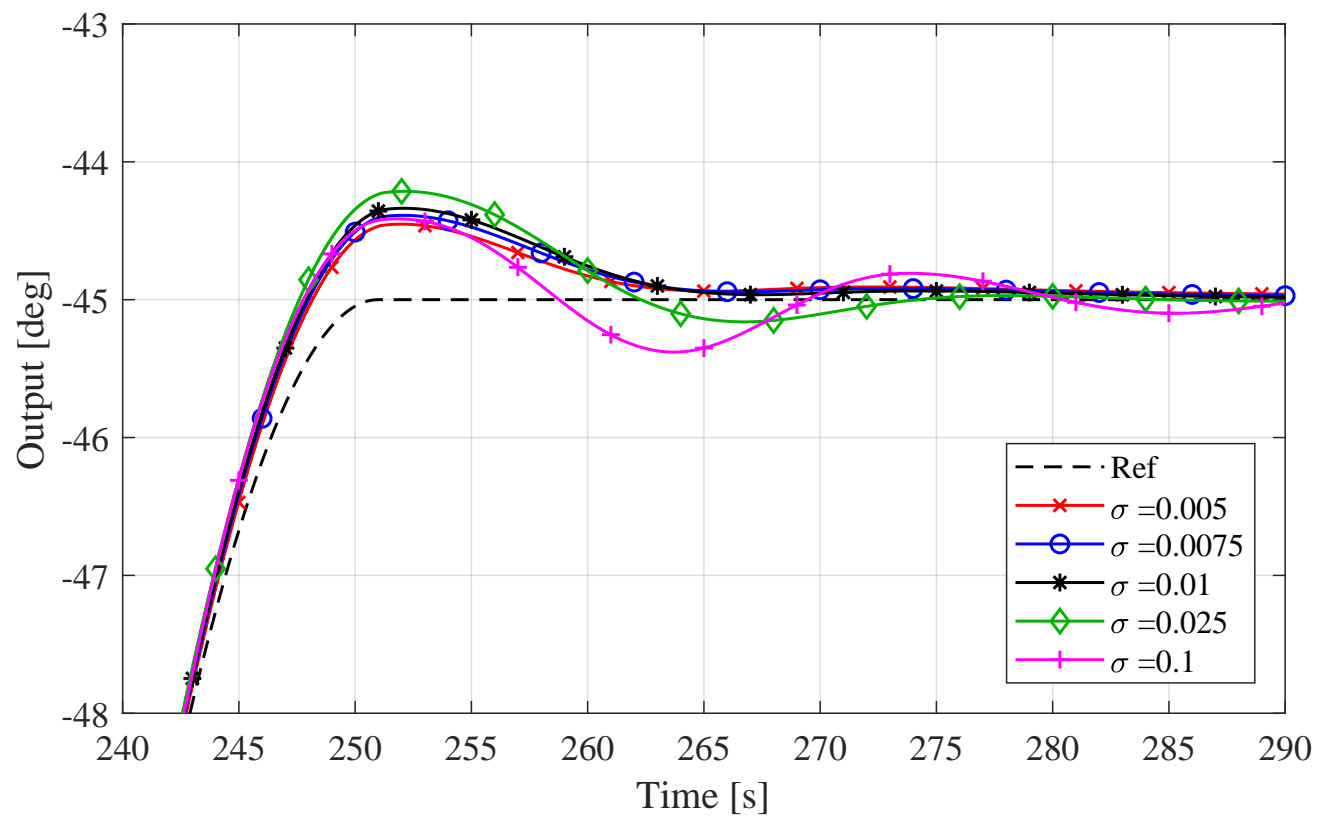

Figure 5.18: Effect of $\sigma$ on closed-loop response.

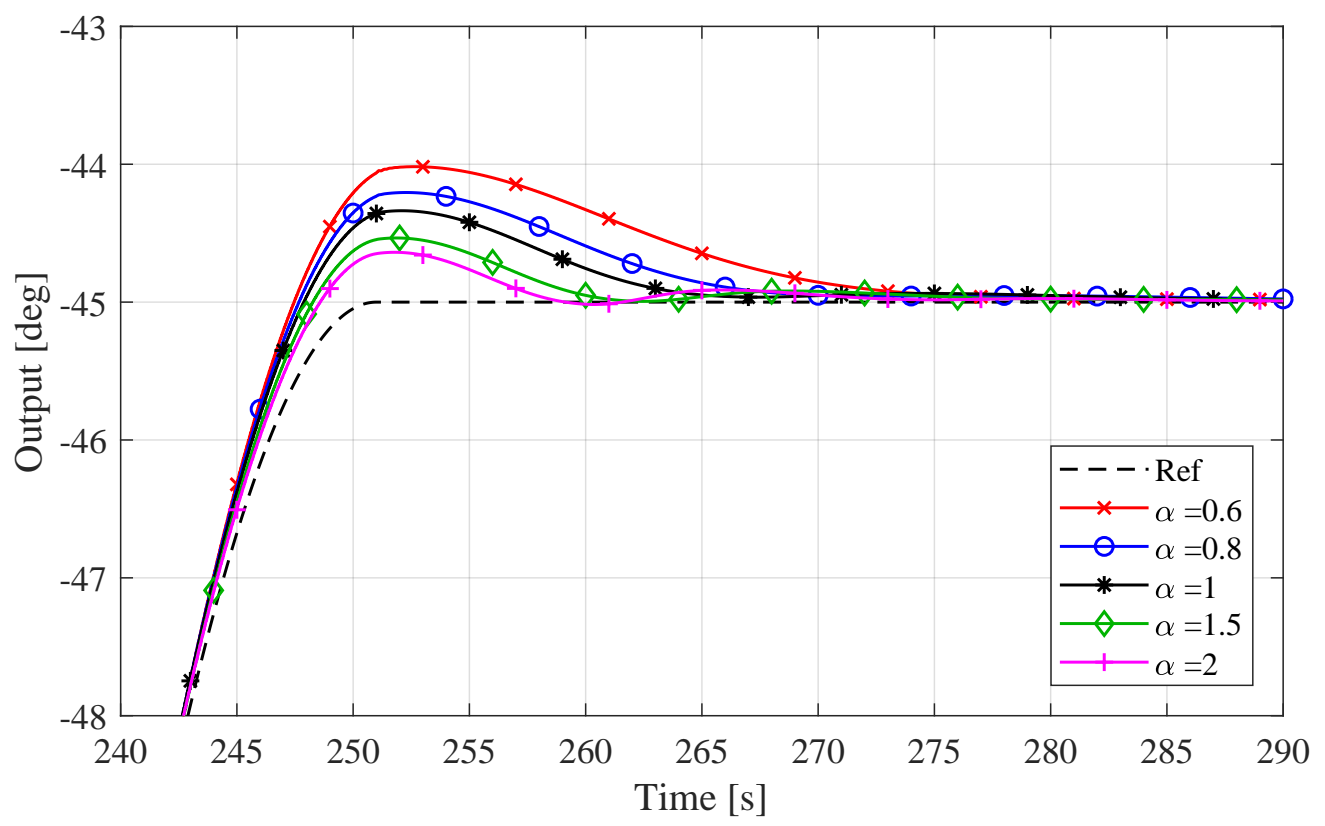

Figure 5.19: Effect of $\alpha$ on closed-loop response.

Of the five tunable parameters, it is evident that $k_{1}$ has the largest impact on the closed-loop response. Figure 5.15 shows that an increasing value of $k_{1}$ will reduce 
overshoot, improve settling time, and dampen the oscillations in the controller. This is due to the fact that $k_{1}$ is a proportional term applied directly to $\omega$ in the virtual controller $\bar{u}_{1}(t)$; which would increase the magnitude of the control response and ultimately make the controller more stiff.

The effect of $k_{2}$ on the system appears to only noticeably affect the overshoot. Unlike $\bar{u}_{1}(t)$, the virtual controller $\bar{u}_{2}(t)$ is solely a function of $k_{2}$ and $\omega$, which is effectively a PI controller. One would expect that as the proportional and integral gains of a controller are simultaneously increased, the overshoot and settling time would be affected in the opposite way. However, since this is not an isolated PI controller, and it is coupled with many additional adaptive parameters, its effect on the system response is less intuitive.

Next, the effect of increasing $\delta$ appears to improve the settling time and reduce overshoot at the expense of introducing an oscillatory response in the system (Fig. 5.17). This is because $\delta$ directly affects the adaptation rates of $\dot{\hat{\Delta}}_{n}^{*}(t), \dot{\hat{\chi}}_{1}(t)$, and $\dot{\hat{\chi}}_{2}(t)$, which ultimately result in determining how quickly the parameters converge, and how sensitive they become to error.

With reference to the effects of $\sigma$ in Fig. 5.18, lower values tend to yield a more consistent performance with little effects on the overshoot and settling time. However as it is increased, the signal becomes less stable and has an oscillatory nature. Since $\sigma$ is a corrective term to mitigate parameter divergence, it is logical that an increase in these values would induce oscillations. However, $\sigma$ cannot be arbitrarily set as a small value, otherwise the adaptive parameters may diverge and drive the controller unstable.

Finally, $\alpha$ tends to increase overshoot, increase settling time, and dampen oscillations as it is decreased. Equation 4.20 would suggest the opposite to be true since $\alpha$ is inversely applied directly to the rate of error. However, as was mentioned earlier, the many coupled terms within this control algorithm render intuition invalid; hence the importance of this sensitivity analysis. 


\subsubsection{Simulation Performance of AB Controller}

With a better understanding of the $\mathrm{AB}$ controller, the gains for the simulated system were selected based on the key observations made in the previous section. The final values for the controller gains are $k_{1}=10, k_{2}=7, \delta=0.008, \sigma=0.01$, and $\alpha=1$, where Fig. 5.20 highlights the performance of the closed-loop system.
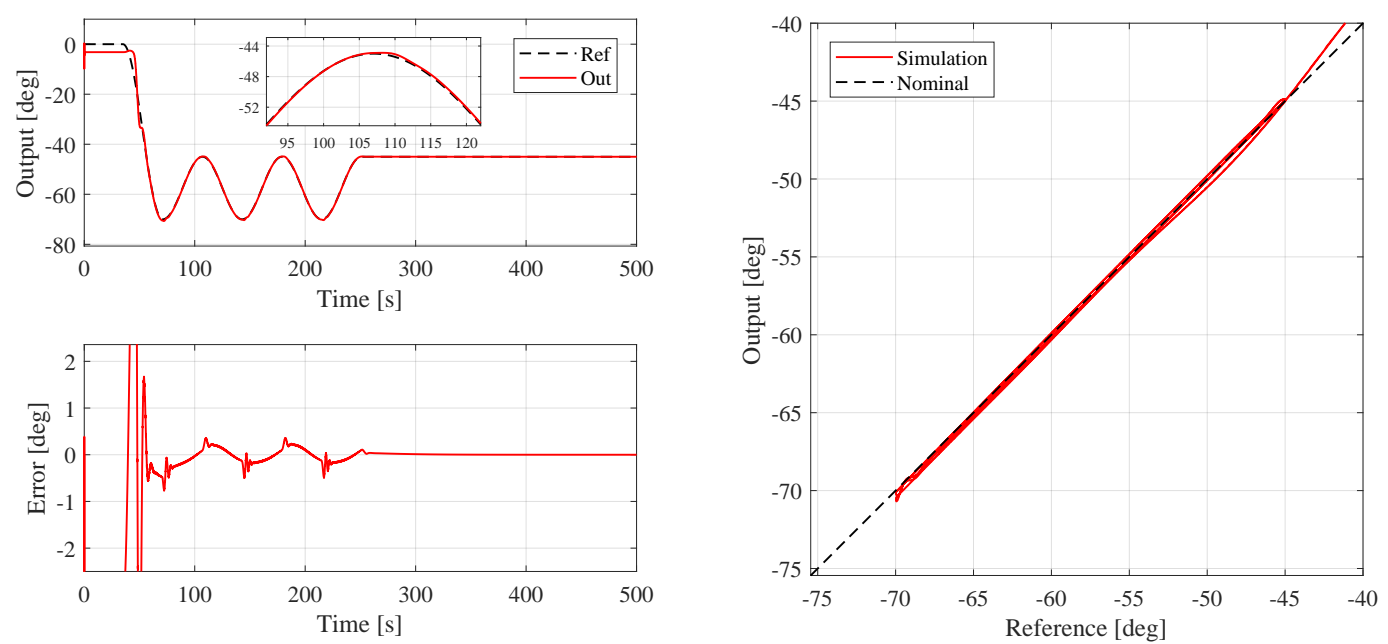

Figure 5.20: Simulated LP joint performance under AB control. Top: actual and desired proximal link angular trajectory, bottom: trajectory tracking error, and right: hysteresis loop of transient phase.

From these figures, is clear that the AB controller greatly out-performs the uncontrolled and PID controlled system. However, Fig. 5.20 shows that the controller takes a much longer time to converge to the reference signal, so caution must be exercised when this controller is implemented in practice. To mitigate this problem, the controller should be given a "warm-up" period to allow $\hat{\Delta}_{n}^{*}(t), \hat{\chi}_{1}(t)$, and $\hat{\chi}_{2}(t)$ converge before actual operation. Once these parameters have converged, the AB controller demonstrates excellent tracking performance, with a maximum error of less than 0.5 degrees. Furthermore, the transient phase delay is nearly completely eliminated, since there is a negligible width to the hysteresis loop.

An interesting property of this controller is that there is a spike in the error whenever there is a direction change in reference signal, as highlighted in Fig. 5.20. This phenomenon also appeared in the simulations presented by Do [45], and could 
not be eliminated.

Though the AB controller demonstrates a superior performance in simulation, it is recognized that this may not be the case in experiment. Since the simulation is using the exact model in which the controller was derived from, it may have difficulty reaching this level of performance in the presence of unmodelled phenomenon such as joint friction, vibrations, and joint backlash, among others.

\subsection{Implementation of $\mathcal{L}_{1}$ Control}

With reference to Fig. 4.4, it can be seen that the inputs to the $\mathcal{L}_{1}$ controller are the reference signal $r(t)$, and the measured state vector $\mathbf{x}(t)$. This implies that the general control architecture shown in Fig. 5.1 must be modified, since it feeds the error signal directly into the controller block. This is done by simply removing the error signal, and directly feeding the plant states and reference signal into the control block, as seen in Fig. 5.21.

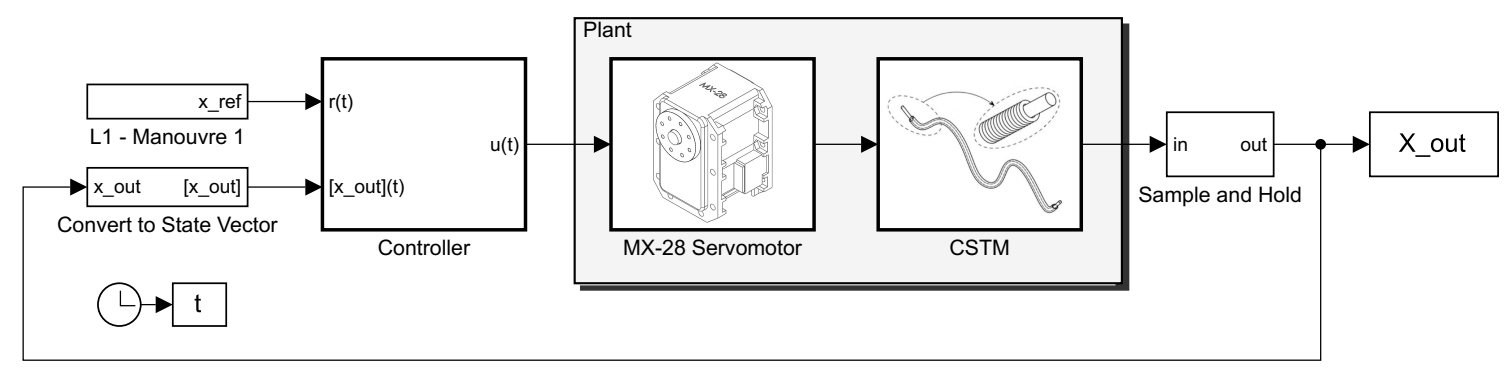

Figure 5.21: Modification to system architecture for $\mathcal{L}_{1}$ controller.

Within the controller block, the algorithm architecture given in Fig. 4.4 was implemented as per Fig. 5.22; where the "Control Law" block executes Eq. (4.46), the "State Predictor" block solves the state dynamics in Eq. (4.38), and the "Adaptive Laws" block solves the differential equations for the parameter estimates from Eqs. (4.39) to (4.41). 


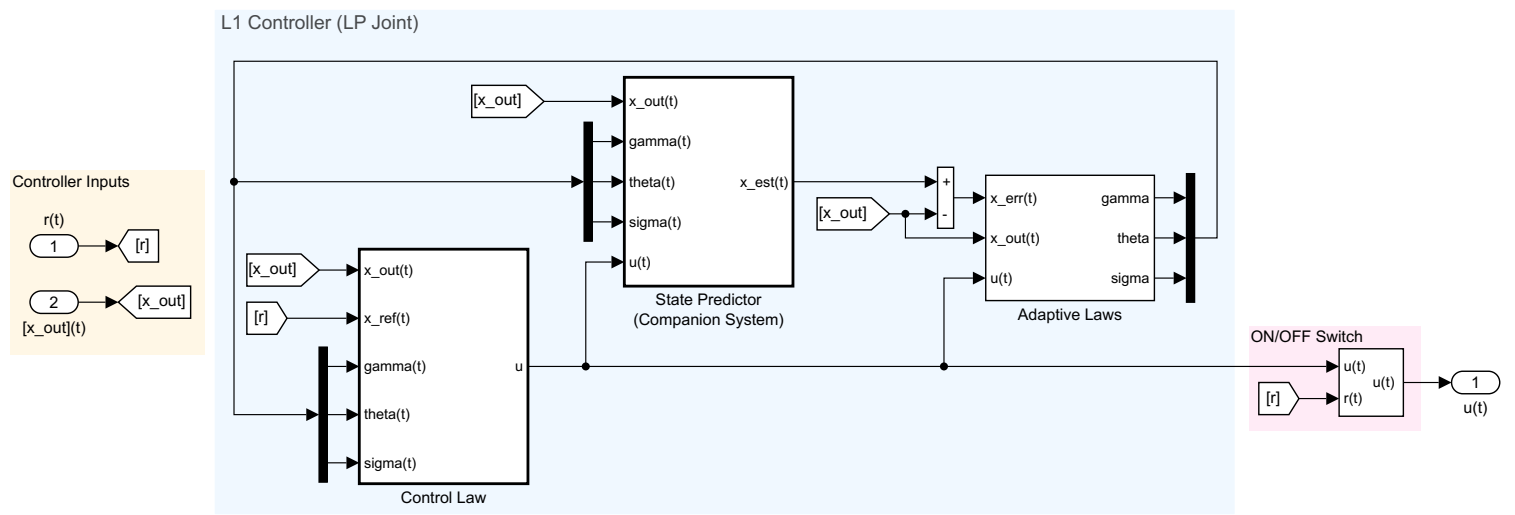

Figure 5.22: Simulink implementation of the $\mathcal{L}_{1}$ controller.

The diagram also contains an ON/OFF switch for the control signal, such that the reference signal can be passed directly to the plant in the absence of control. In order to initialize the simulation with all user defined parameters, the script RUN_L1_LP.mat in Appendix B.5 is run.

\subsubsection{Sensitivity Analysis of $\mathcal{L}_{1}$ Parameters}

Due to its complexity, tuning the $\mathcal{L}_{1}$ controller is rather tedious. In fact, there are no analytical tuning methods of the $\mathcal{L}_{1}$ controller that exist without running a separate optimization routine to determine the optimal gains and filter parameters $[59,61]$. Furthermore, techniques such as these require knowledge of the plant dynamics to solve numerically, which ultimately rules it out as a tuning method for the experiment since the full system dynamics are not modelled. Therefore, a qualitative approach was taken to determine the effects of each user defined parameter for the LP joint simulation by means of a sensitivity study. It is important to note that the results of this analysis may not be valid for a different system, so the general guidelines presented here are only assumed to be relevant to this work.

Prior to performing the sensitivity study, the user defined parameters of the controller must be identified. These parameters include: $\mathbf{A}_{m}, \mathbf{B}, \Gamma, k, D(s)$, and the boundaries on the adaptive parameters, $\left[\gamma_{l}, \gamma_{u}\right], \Delta_{o}$, and $\Theta$. To simplify the tuning process, there are several assumptions and deductions that can be made.

First, the response of the companion model was chosen to follow a critically 
damped system with a settling time of $t_{s}$ and DC gain of 1 . Let us define the form of the system matrices, such that the plant transfer function $\mathbf{G}_{p}(s)$ can be determined by Eq. (5.3).

$$
\begin{gathered}
\mathbf{A}_{m}=\left[\begin{array}{cc}
0 & 1 \\
a_{21} & a_{22}
\end{array}\right] \quad \mathbf{B}=\left[\begin{array}{c}
0 \\
b_{21}
\end{array}\right] \\
G_{p}(s)=\frac{b_{21}}{s^{2}+a_{22} s+a_{21}}
\end{gathered}
$$

This leads to the definition of the plant natural frequency $\omega_{n}$, damping ratio $\zeta$, and the DC gain of the system $K$ at $G_{p}(0)$.

$$
\begin{aligned}
\omega_{n} & =\sqrt{a_{21}} \\
\zeta & =a_{22} /\left(2 \omega_{n}\right) \\
K & =b_{21} / a_{21}
\end{aligned}
$$

By approximating the $2 \%$ settling time of a critically damped system to be $t_{s}=$ $5.8 /\left(\omega_{n}\right)$, the damping ratio to be $\zeta=1$, and the DC gain to be $K=1$, the following relationships are defined for the matrix elements $a_{21}, a_{22}$, and $b_{21}$ respectively. Note that with these definitions, the matrices $\mathbf{A}_{m}$ and $\mathbf{B}$ are now simultaneously determined by simply selecting the desired settling time of the reference model $t_{s}$, and it will guarantee that $\mathbf{A}_{m}$ is Hurwitz and controllable.

$$
\begin{aligned}
& a_{21}=\left(5.8 / t_{s}\right)^{2} \\
& a_{22}=2 \sqrt{a_{21}} \\
& b_{21}=a_{21}
\end{aligned}
$$

Secondly, the adaptive parameter boundaries $\left[\gamma_{l}, \gamma_{u}\right], \Delta_{o}$, and $\Theta$ are relatively simple to identify. Through iteration, these values can be set in such a way that the values of $\hat{\gamma}(t), \hat{\boldsymbol{\theta}}(t)$, and $\hat{\boldsymbol{\sigma}}(t)$ are not saturated by their boundaries during the 
operation of the controller. If these parameters were to be saturated, the performance of the controller would become compromised and could lead to instability.

With these basic assumptions made, the key parameters to tune include $\Gamma, k$, $D(s)$, and $t_{s}$, which dramatically reduces the complexity of the tuning process. Of these parameters, the most complex to select is the filter $D(s)$, since its order and coefficients are entirely unknown. As mentioned previously, there are optimal ways to selected this filter, though this solution requires exact knowledge of the plant and can therefore not be applied [61]. For the sake of simplicity, it will be assumed that $D(s)$ is a low-pass filter with the form given in Eq. (5.10).

$$
D(s)=\frac{1}{\psi s+1}
$$

where $\psi$ is a positive scalar which controls the bandwidth of the filter. With there being four individual scalars to determine, the tuning process of the controller is greatly simplified.

To initialize the sensitivity study, all tunable parameters were set to $\Gamma=k=$ $t_{s}=\psi=1$ and individually incremented by small values to isolate their effect on the system response. Specifically, the effects on the output response in the "hold" phase of Manoeuvre 1 is of interest, as it captures the transient performance and steady state performance simultaneously. The effects of the tunable parameters on the simulated LP joint angle response are captured in Fig. 5.23 to 5.26 for $\Gamma, k, t_{s}$, and $\psi$ respectively, where the black-asterisk marked curve represents the common response between all figures. 


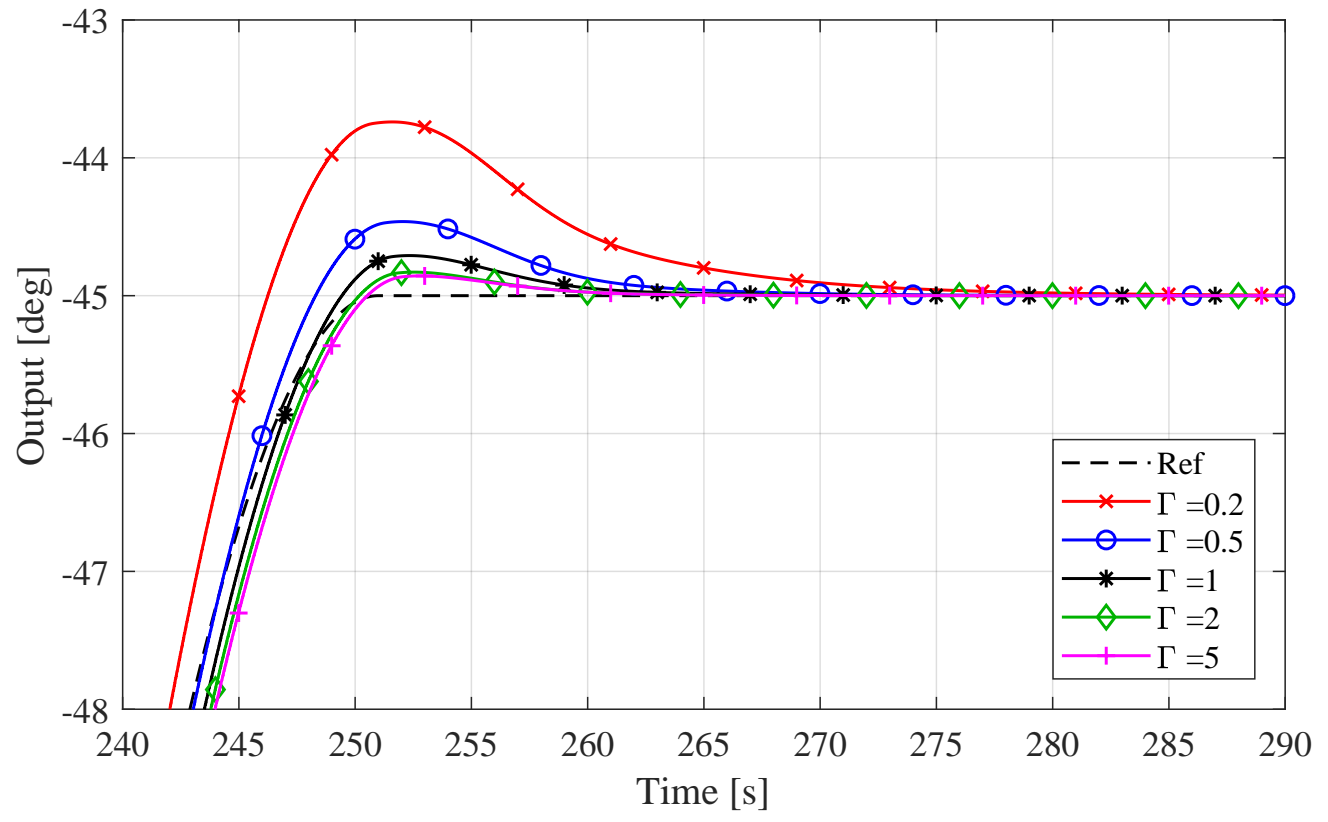

Figure 5.23: Effect of $\Gamma$ on closed-loop response.

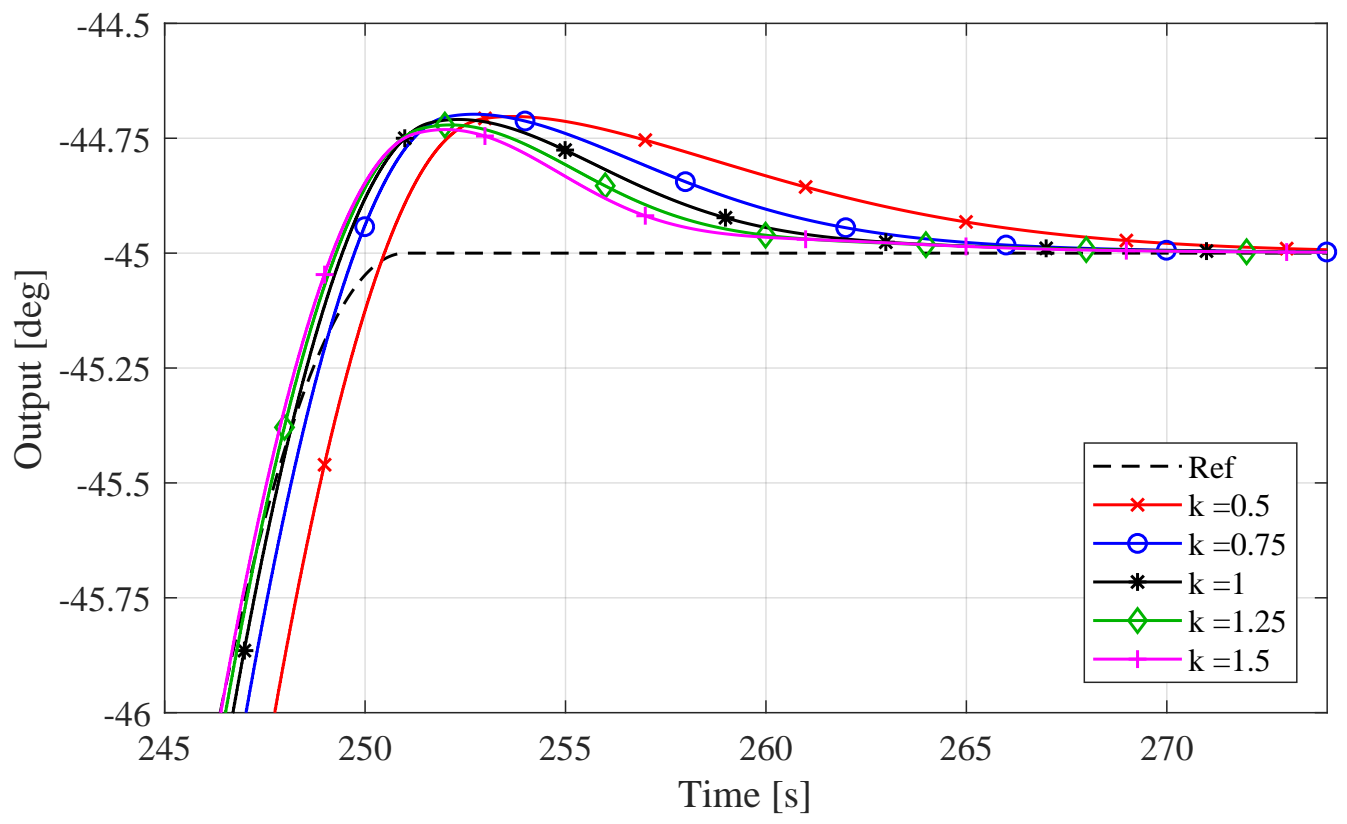

Figure 5.24: Effect of $k$ on closed-loop response. 


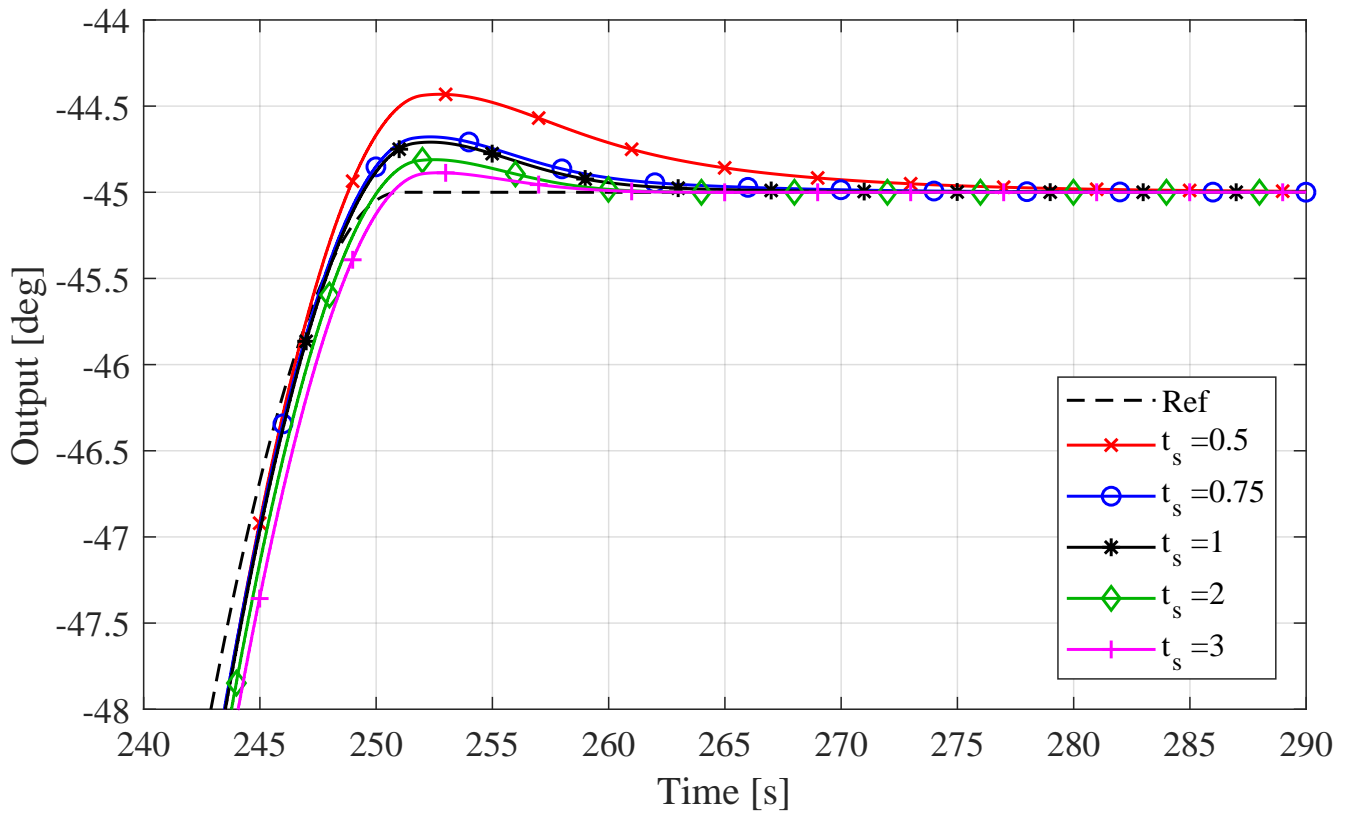

Figure 5.25: Effect of $t_{s}$ on closed-loop response.

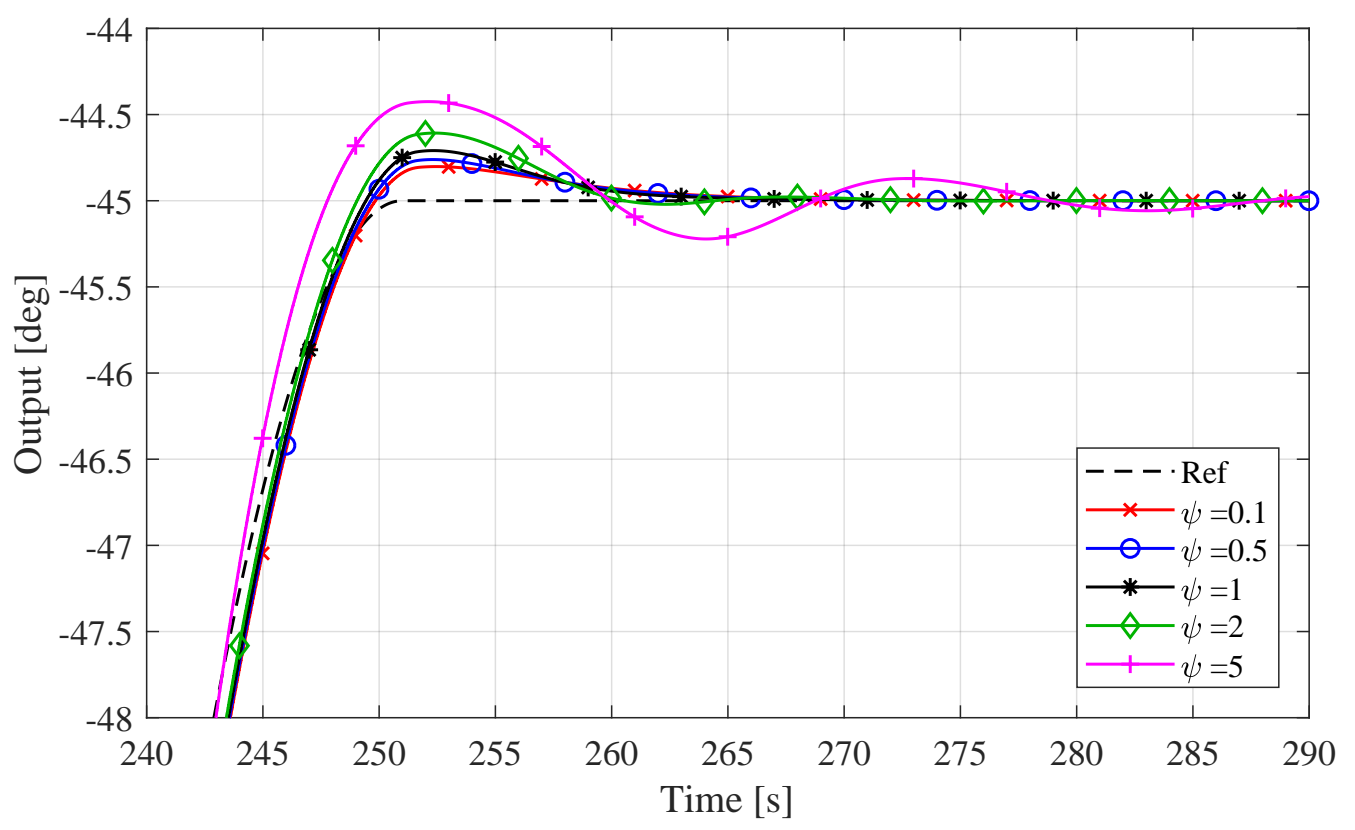

Figure 5.26: Effect of $\psi$ on closed-loop response.

First, let us investigate the effect of $\Gamma$. From 5.23, it can be seen that as one increases $\Gamma$, the controller becomes stiffer by reducing overshoot and settling time. 
This is a logical outcome since the learning rate is a scalar gain on the adaptation laws, making $\hat{\gamma}(t), \hat{\boldsymbol{\theta}}(t)$, and $\hat{\boldsymbol{\sigma}}(t)$ more sensitive to the prediction error $\tilde{\mathbf{x}}(t)$. A key thing to note here is that since the selection of $\Gamma$ will affect the adaptation rates directly, the bandwidth of the filter must also be adjusted to filter out high frequency oscillations produced by the controller.

With reference to Fig. 5.24, the effect of $k$ has a similar trend. It can be seen that the primary effect on the system response is that the control input tends to become stiffer and oscillatory by increasing the value of $k$, while maintaining roughly the same overshoot. This is also logical since it is acting as a straight proportional gain to the control input, which is known to exhibit these effects for most controllers. Additionally, an increasing $k$ exhibits a decrease in the phase lag of the response, which is also to be expected.

Next, the variation of $t_{s}$ is presented by Fig. 5.25. Interestingly, as the settling time of the companion model increases, the actual settling time of the closed-loop system is reduced, along with its overshoot. Upon further inspection of the adaptation laws in Eqs. (4.39) to (4.41), and by recalling that the Lyapunov matrix $\mathbf{P}$ must decrease as $\mathbf{A}_{m}$ increases, this is in fact a reasonable outcome. Since $\mathbf{P}$ directly affects the adaptation laws, and an increase in $t_{s}$ would result in a decrease in $\mathbf{P}$, it is expected to have a similar effect on the response as $\Gamma$.

Lastly, the selection of the filter coefficient $\psi$ primarily affects the overshoot and the oscillations in the response. This is due to the fact that as $\psi$ changes, the cut-off frequency and gain of the filter are changed simultaneously, which would exhibit these effects. These results could be very different for a different form of filter, however, and the scale of the analysis required to determine these effects was deemed to be outside the scope of this work.

With these key observations, the controller can be tuned for this system by systematically changing each parameter and observing the response. It is important, however, to recognize that all of these parameters are coupled in some way, so these tuning rules cannot be applied naively. Furthermore, the stability condition defined in Theorem 2 must be met to ensure a stable closed-loop performance. 


\subsubsection{Simulation Performance of $\mathcal{L}_{1}$ Controller}

With the general tuning process outlined, the controller parameters were adjusted in attempts to improve its performance. After a process of trial and error, the values selected were $\Gamma=0.8, k=1.12, t_{s}=1.5$, and $\psi=1 / 3$, where the performance is presented in Fig. 5.27. Note that these gains meet the stability condition with $\|G(s)\|_{\mathcal{L}_{1}} L=0.8936$, and that the controller was not active until $t=35$ seconds.
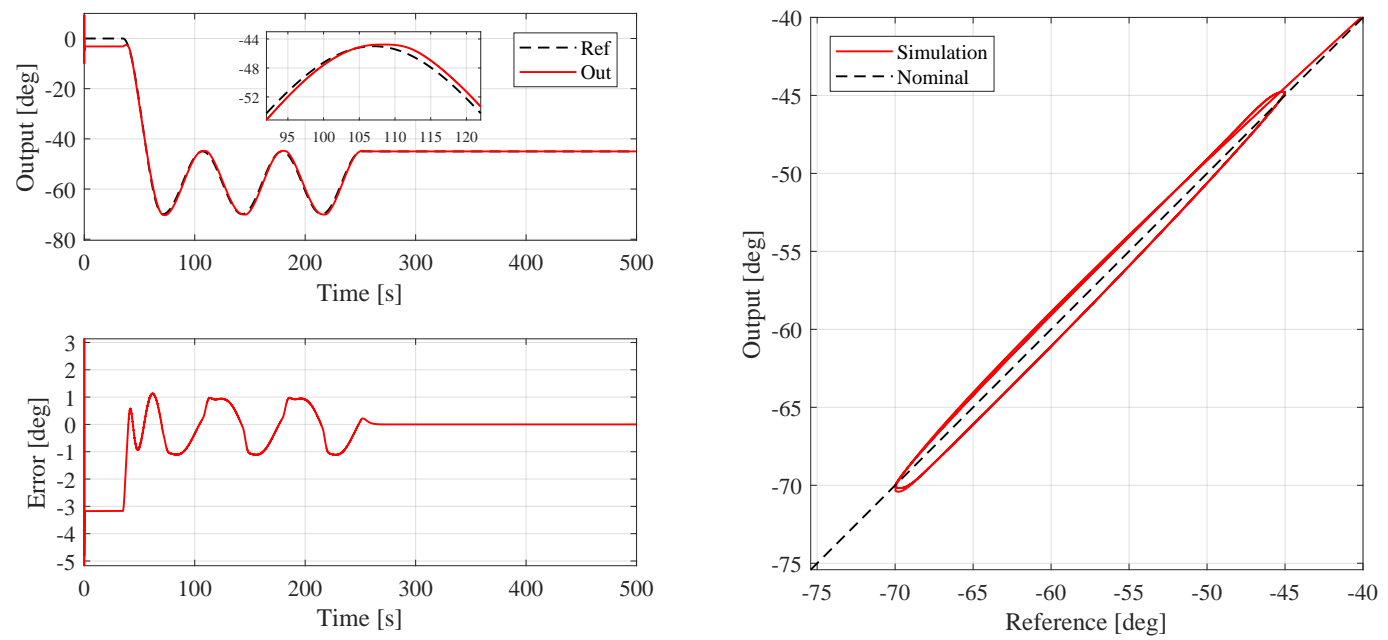

Figure 5.27: Simulated LP joint performance under $\mathcal{L}_{1}$ control. Top: actual and desired proximal link angular trajectory, bottom: trajectory tracking error, and right: hysteresis loop of transient phase.

In general, the $\mathcal{L}_{1}$ controller performed even better than the PID controller, having the maximum joint error of roughly $1 \mathrm{deg}$ during the entire manoeuvre, with the error converging to zero during the "hold" portion of the manoeuvre. Upon inspection of the hysteresis loop, it is evident that the phase delay was improved over the PID controlled case, however, was degraded with respect to the AB controller. Additionally, there is more overshoot at the boundaries of the hysteresis loop than the previous controllers, which can prove to be problematic for robotic manoeuvres. Despite numerous attempts, this phenomenon could not be completely removed in simulation. In general, this controller has excellent steady state performance, and an acceptable performance during the transient phase. 


\section{Chapter 6}

\section{Experimental Results and Discussion}

Here, the experimental results of the fully controlled gripper are presented for a select set of case studies. First, Section 6.1 presents the details of the selected cases studies, and outlines their implementation on the SPOT platform. Then, subsequent sections present the experimental results and discuss their performance accordingly for the LP joint. Each section is divided by control case, where Section 6.2 shows the performance of the uncontrolled system, Section 6.3 gives the performance of the baseline PID controller, the AB controlled gripper is given in Section 6.4, and finally, the $\mathcal{L}_{1}$ controller is given in Section 6.5.

\subsection{Overview of Experiments}

Based on the required grip configurations covered in Section 2.1, there are three manoeuvres developed for each link; a pure sine wave manoeuvre to capture the transient performance, and two warm-up and hold manoeuvres with a transient phase and a steady state phase. The details of each of these manoeuvres are given in Table 6.1 .

Table 6.1: Description of selected reference signals.

\begin{tabular}{|c|c|c|c|c|}
\hline Joint & Manoeuvre & For Grip & Steady State $\theta$ & Transient Phase \\
\hline \hline Prox. & Sine Wave & All & None & $-70 \leq \theta_{p} \leq-45 \mathrm{deg}$ \\
\hline Prox. & Manoeuvre 1 & GC-B, GC-C & $\theta_{p}=-45 \mathrm{deg}$ & $-70 \leq \theta_{p} \leq-45 \mathrm{deg}$ \\
\hline Prox. & Manoeuvre 2 & GC-A & $\theta_{p}=-90 \mathrm{deg}$ & $-70 \leq \theta_{p} \leq-45 \mathrm{deg}$ \\
\hline Dist. & Sine Wave & All & None & $30 \leq \theta_{d} \leq 60 \mathrm{deg}$ \\
\hline Dist. & Manoeuvre 1 & GC-C & $\theta_{d}=90 \mathrm{deg}$ & $30 \leq \theta_{d} \leq 60 \mathrm{deg}$ \\
\hline Dist. & Manoeuvre 2 & GC-A, GC-B & $\theta_{d}=0 \mathrm{deg}$ & $30 \leq \theta_{d} \leq 60 \mathrm{deg}$ \\
\hline
\end{tabular}


All of the reference signals are initialized and held at $r(t)=0 \operatorname{deg}$ for $0 \leq t \leq$ 35 seconds to allow the ground truth system to boot and connect to the RP3 onboard the SPOT. After this 20 seconds, the reference signals are then passed to the controller blocks, where an additional internal switch controls the "ON/OFF" state of the controller. Once the controller has been activated, the transient phase of the manoeuvre begins, where all oscillations in the reference signal are supplied at a frequency of $5 \mathrm{deg} / \mathrm{s}$. The purpose of the transient phase in Manoeuvres 1 and 2 is to allow the adaptive parameters of the $\mathrm{AB}$ and $\mathcal{L}_{1}$ controllers to reach a quasi-steady state before attempting the "hold" portion of the manoeuvre. The experiment is then terminated after 500 seconds, and all data is sent to the command computer for later analysis.

To fully characterize the performance of the gripper under the selected control scenarios, there were several case studies performed. Aside from testing the controller's performance with the expected operating range, it is also important to assess the controller's performance under varying conditions of the CSTM. Thus, the experiments were performed with a straight arm configuration for the minimum friction case $\left(\theta_{S}=\theta_{E}=\theta_{W}=0 \mathrm{deg}\right)$, a fully bent arm to maximize the CSTM friction $\left(\theta_{S}=\theta_{E}=\theta_{W}=90 \mathrm{deg}\right)$, and a compound fully-articulated dynamic friction experiment with all gripper links and arm links in motion simultaneously $(0 \leq \theta \leq 90 \mathrm{deg}$ for all arm joint angles). The latter of these cases was only performed with the Sine Wave manoeuvre since the steady state performance will be captured in Manoeuvres 1 and 2 in the other arm configurations.

With these arm configurations, manoeuvres, and control cases for all the links, there were 64 cases run in total for 5 trials each to give a preliminary indication of repeatability. Due to the high number of cases, this section will focus primarily on the LP joint for the scenarios outlined in Table 6.2. For all other cases, please refer to Appendix C.

\subsection{No Control}

Prior to analysing the performance of the gripper under the selected control cases, the performance of the plant must first be discussed. For this scenario, the control 
Table 6.2: Key scenarios of LP joint covered in this Chapter.

\begin{tabular}{|c|c|c|c|}
\hline Joint & Manoeuvre & Control Cases & Arm Config. \\
\hline \hline LP & Manoeuvre 1 & All (4) & Straight - static \\
\hline LP & Manoeuvre 1 & All (4) & Bent - static \\
\hline LP & Sine Wave & All (4) & Full range - dynamic \\
\hline
\end{tabular}

algorithms were switched off to allow the reference signals to be passed to the plant directly. First, let us examine the performance of the LP joint in the straight arm configuration as per Fig. 6.1.
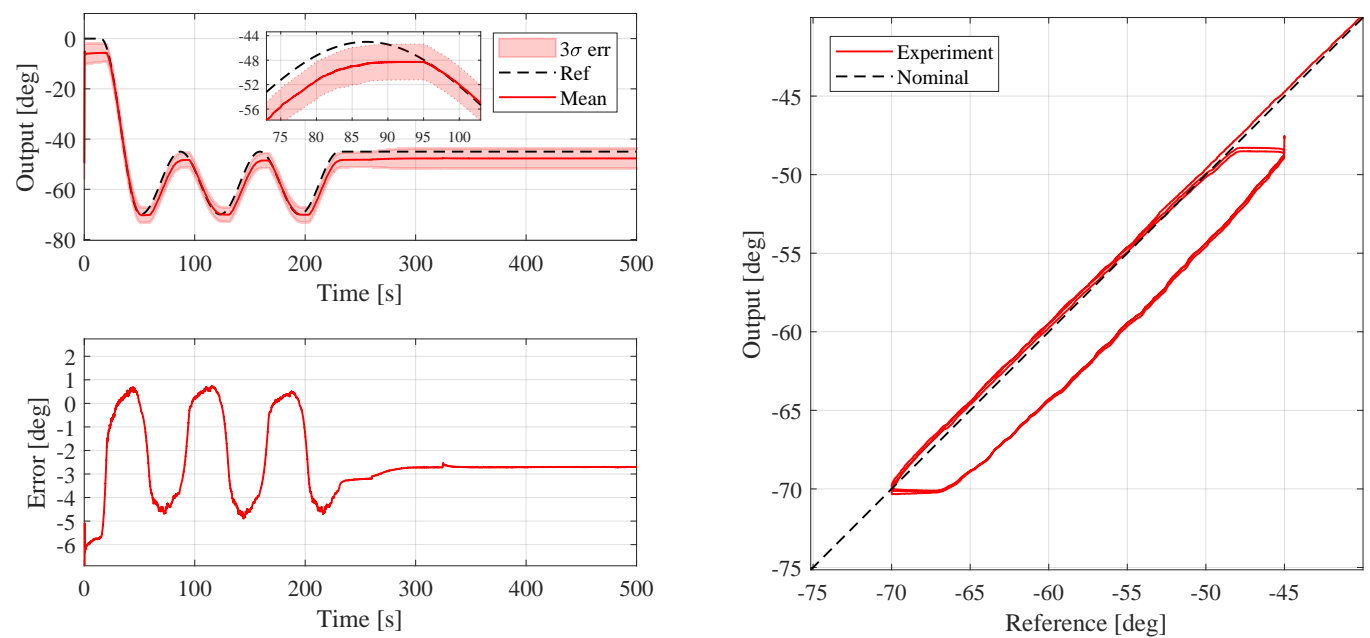

Figure 6.1: LP joint performance in straight arm configuration under no control. Top: actual and desired proximal link angular trajectory, bottom: mean trajectory tracking error, and right: mean hysteresis loop of transient phase.

From the figure, it can be observed that the tracking performance of the uncontrolled plant is quite poor; where the overall error nearly reaching $5 \mathrm{deg}$, with an average phase delay of 2.48 seconds (neglecting dead-zones). Furthermore, over the 5 trials performed, the $3 \sigma$ of the standard error indicates that the plant has some stochastic elements which cause a varying performance. This may be due to the fact that the initial conditions will not be exactly the same between trials, which means the friction through the sheath will vary. Despite attempts to improve its repeatability, the problem persisted. This suggests that this is simply the behaviour of the 
plant. This is further reinforced by all other uncontrolled cases outlined in Appendix C.1.

When comparing the experimental results in Fig. 6.1 to the simulated plant performance for the same manoeuvre in Fig. 5.11, the general trend for the tracking error is similar, however, there is an interesting difference with the hysteresis loop. In experiment, the CSTM appears to have more prominent dead-zones when a change in direction is performed, as evident by the flat horizontal portions in the loop. However, the modified Bouc-Wen model has a more rounded/gradual transition into the slopes of the loop. This indicates that there is a difference between the reference model of the CSTM and the actual plant; which is to be expected. Since this is the case, it is hypothesized that the indirect $\mathrm{AB}$ controller will perform worse (in general) than it did in the simulated environment since the plant does not perfectly match the model in which the controller was designed from. Now, let us examine how the plant behaves for the same manoeuvre in the bent arm configuration (maximum friction), given by Fig. 6.2.
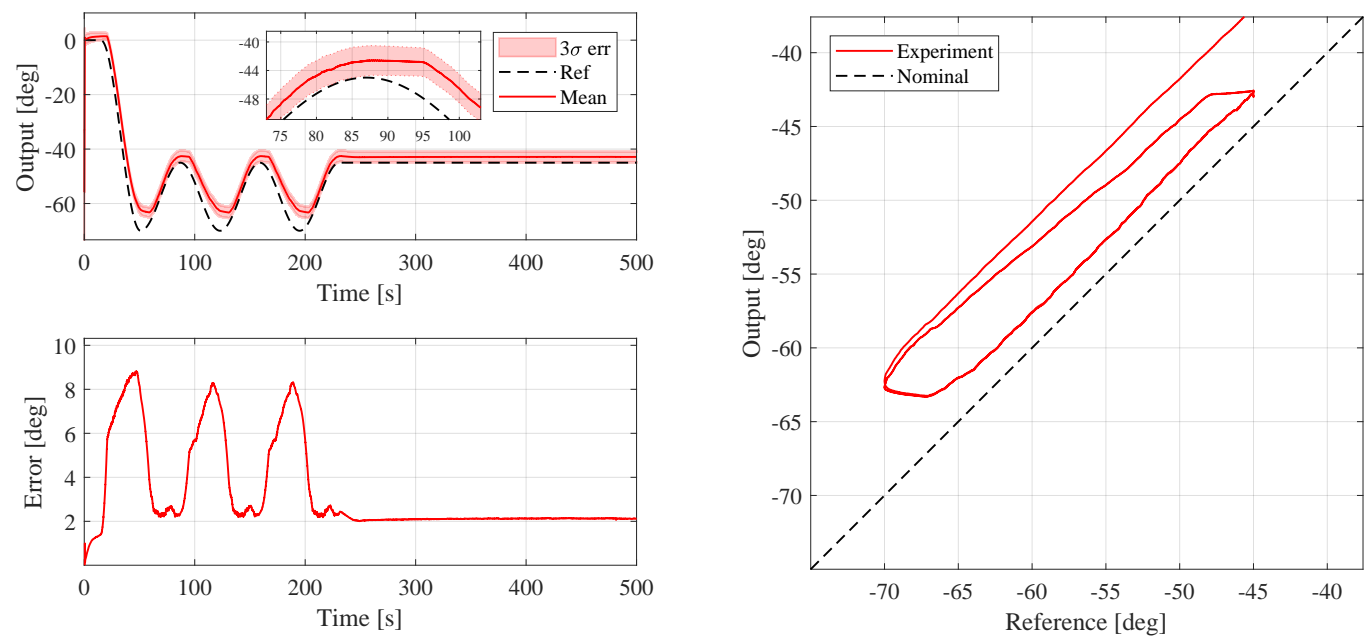

Figure 6.2: LP joint performance in bent arm configuration under no control. Top: actual and desired proximal link angular trajectory, bottom: mean trajectory tracking error, and right: mean hysteresis loop of transient phase.

Immediately, one can notice that the mean trajectory is entirely above the reference signal, with a maximum error of roughly $9 \mathrm{deg}$. This is a logical outcome because 
as the friction increases in the CSTM, the torsion spring will reach static equilibrium with the cable tension sooner than it would have in the straight arm configuration (recall that approaching a negative joint angle means the gripper is opening). This phenomenon also has an effect on the shape of the hysteresis loop, where the gripper becomes less responsive as the reference signal approaches -70 deg; as is evident by the change in width of the loop. Aside from these key differences, the overall repeatability of the gripper under maximum friction conditions is similar as it is when in the straight arm configuration, and the average lag in the response is 3.848 seconds. Finally, the performance of the LP joint under time varying CSTM friction is shown in Fig. 6.3.
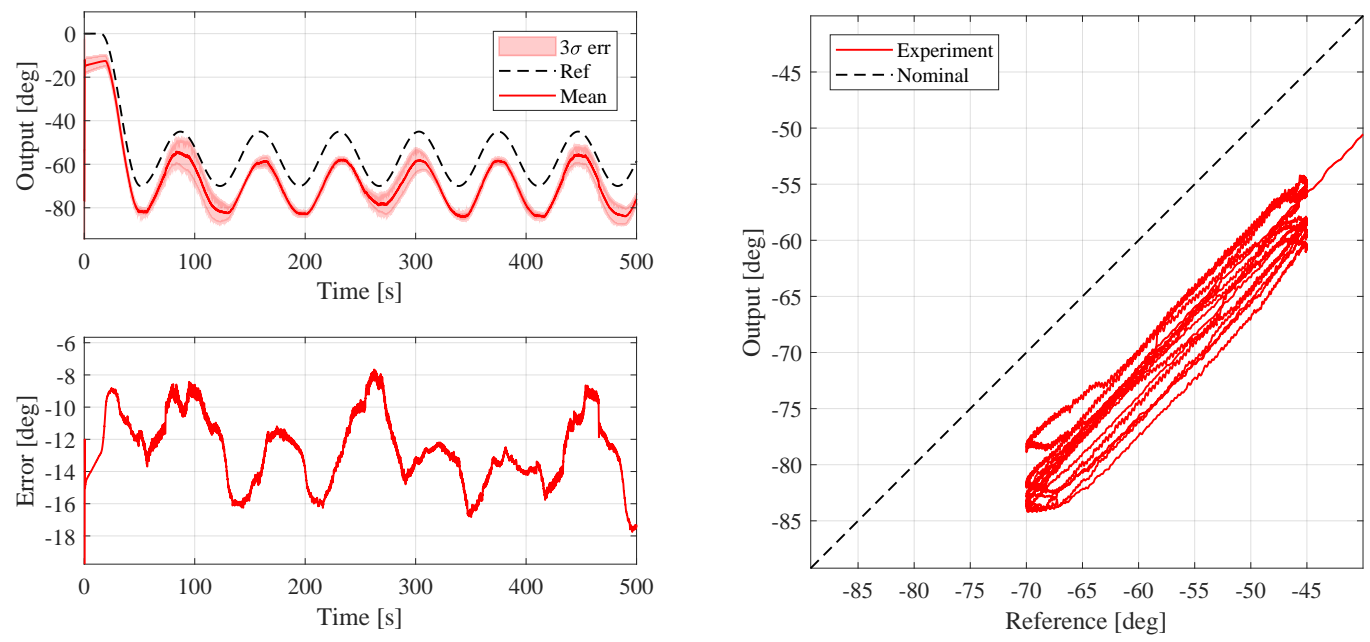

Figure 6.3: LP joint performance with moving arm under no control. Top: actual and desired proximal link angular trajectory, bottom: mean trajectory tracking error, and right: mean hysteresis loop of transient phase.

In this scenario, the tracking performance of the uncontrolled plant is extremely poor, with a mean error reaching as high as $18 \mathrm{deg}$. As expected, as the arm configuration changes, so too does the shape and location of the hysteresis loop. Additionally, the signal appears to exhibit high-frequency oscillations when compared to the static case. This could be a combination of a few factors. One being that during the experiment, it was observed that the CSTM was "jittering" as the arm and gripper were 
actuated simultaneously. This it likely due to the fact that a change in the CSTM curvature will change the value of $L_{1}$ (as per Eq. (2.45)); ultimately determining whether the cable transmission is in a dead-zone state, or a transmission state. As the value of $L_{1}$ shifts past the length of the sheath $L$, then the cable will suddenly move from a dead-zone to a dynamic state, and then quickly return to another dead-zone as the curvature changes.

Furthermore, it was observed that the average trajectory of the plant fell below the reference signal; much further than the previous two arm configurations. This result at first seems peculiar since one would expect that the trajectory would fall between the minimum and maximum friction cases by Fig. 6.1 and Fig. 6.2 respectively. However, this phenomenon may too be a result of the cable being rapidly shifted between static and dynamic states. Since the Bouc-Wen model suggests that the form of the hysteresis loop also depends on the velocity of the tendon (Eq. (4.3)), a rapid change would cause the cable velocity to be greater, which would lead to a further displacement than if the arm were in a static configuration. 


\subsection{PID Control}

In this section, the system performance will be analysed under PID control for the LP joint. With reference to the PID algorithm implementation in Section 5.2, the gains selected for this experiment were $K_{p}=0.5, K_{i}=0.9$, and $K_{d}=0.15$; which were tuned for the straight arm configuration. First, let us examine experimental results for the straight arm configuration shown in Fig. 6.4.
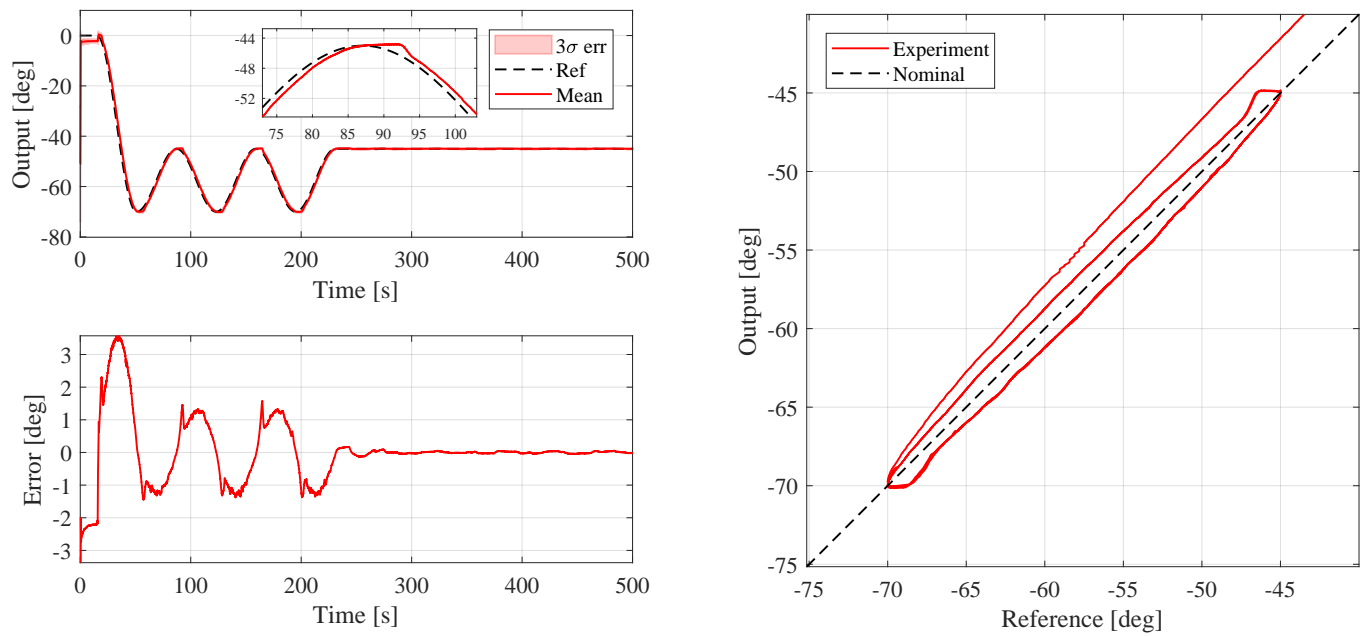

Figure 6.4: LP joint performance in straight arm configuration under PID control. Top: actual and desired proximal link angular trajectory, bottom: mean trajectory tracking error, and right: mean hysteresis loop of transient phase.

Indeed, the overall tracking performance of the gripper is greatly improved by introducing a feedback controller into the system, where the largest error in the transient phase (excluding the drop from the initial state) was approximately 1.5 deg. Additionally, the repeatability of the controlled system has improved greatly, where the $3 \sigma$ error is imperceptible on the plot. With reference to the hysteresis plot, the loop now is centred on the nominal line, and the phase delay has been reduced to an average of 1.15 seconds. This result was expected as per the simulated results presented in Fig. 5.13. In general, the PID controller performance is very similar to what was simulated for the straight arm configuration. Next, the PID performance in the bent arm configuration is given by Fig. 6.5. 

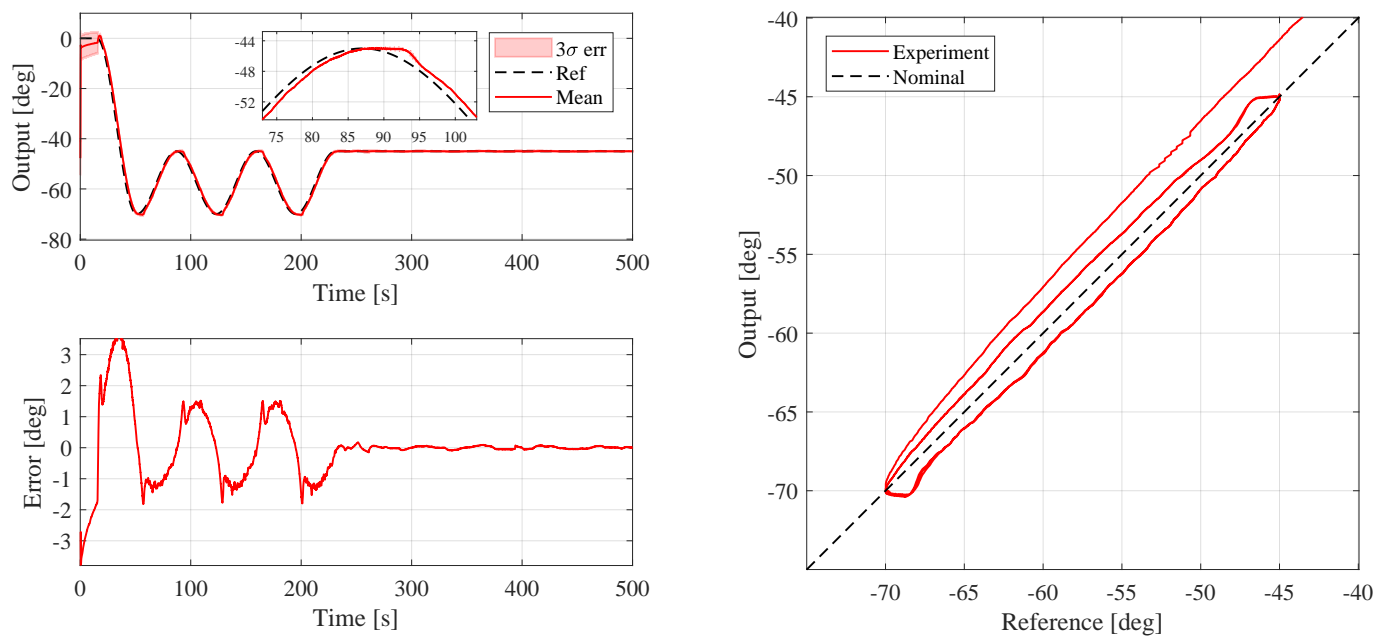

Figure 6.5: LP joint performance in bent arm configuration under PID control. Top: actual and desired proximal link angular trajectory, bottom: mean trajectory tracking error, and right: mean hysteresis loop of transient phase.

The PID controller performs nearly identically as in the straight arm configuration with a few minor differences. Upon careful inspection of the error plot, the bent arm PID case exhibits a slightly greater error in both directions, and the average lag in the response has increased to 1.22 seconds. This result can be justified since the fixed-gain controller must now overcome more friction to reach the reference signal. Fortunately, the integral portion of the PID controller reduces steady state error as a result of the high transmission friction. Now, let us examine the PID controller under a dynamic arm configuration, as per Fig. 6.6. 

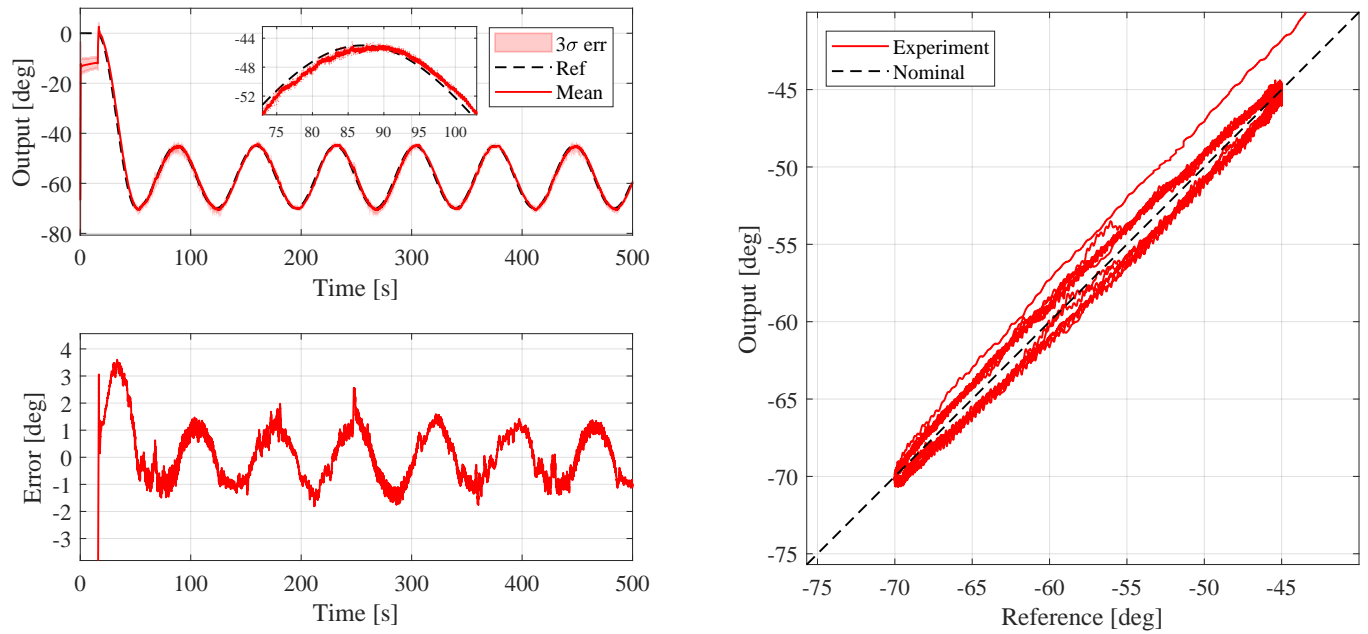

Figure 6.6: LP joint performance with moving arm under PID control. Top: actual and desired proximal link angular trajectory, bottom: mean trajectory tracking error, and right: mean hysteresis loop of transient phase.

Aside from the high-frequency oscillations explained previously, the overall performance is similar to the static arm cases; there is a noticeable phase delay in the system, and the transient error oscillates in a similar fashion. Note that quantifying the phase delay for the dynamic case would be nonsensical due to the presence of high-frequency oscillations. One key thing to note here is that the dead-zones of the system are nearly gone; which is evident by the absence of plateaus in the hysteresis loop, and the zoomed portion of the joint trajectory plot. Secondly, the magnitude of the error is less consistent with time during the transient phase, ranging between roughly 1.0 - $2.0 \mathrm{deg}$. This further demonstrates that the fixed gains of the controller produce less consistent tracking performance when compared to the case it was tuned for due to the dynamic variations of the plant. 


\subsection{AB Control}

Next, the AB controller performance is investigated here. From the tuning guideline presented in Section 5.3.1, the controller gains selected for the experiment include $k_{1}=2, k_{2}=1, \delta_{1}=\delta_{2}=\delta_{3}=0.005, \sigma_{1}=\sigma_{2}=\sigma_{3}=0.01, \epsilon=0.05$ and $\alpha=1$. These gains were tuned in the straight arm configuration, with their performance given in Fig. 6.7.
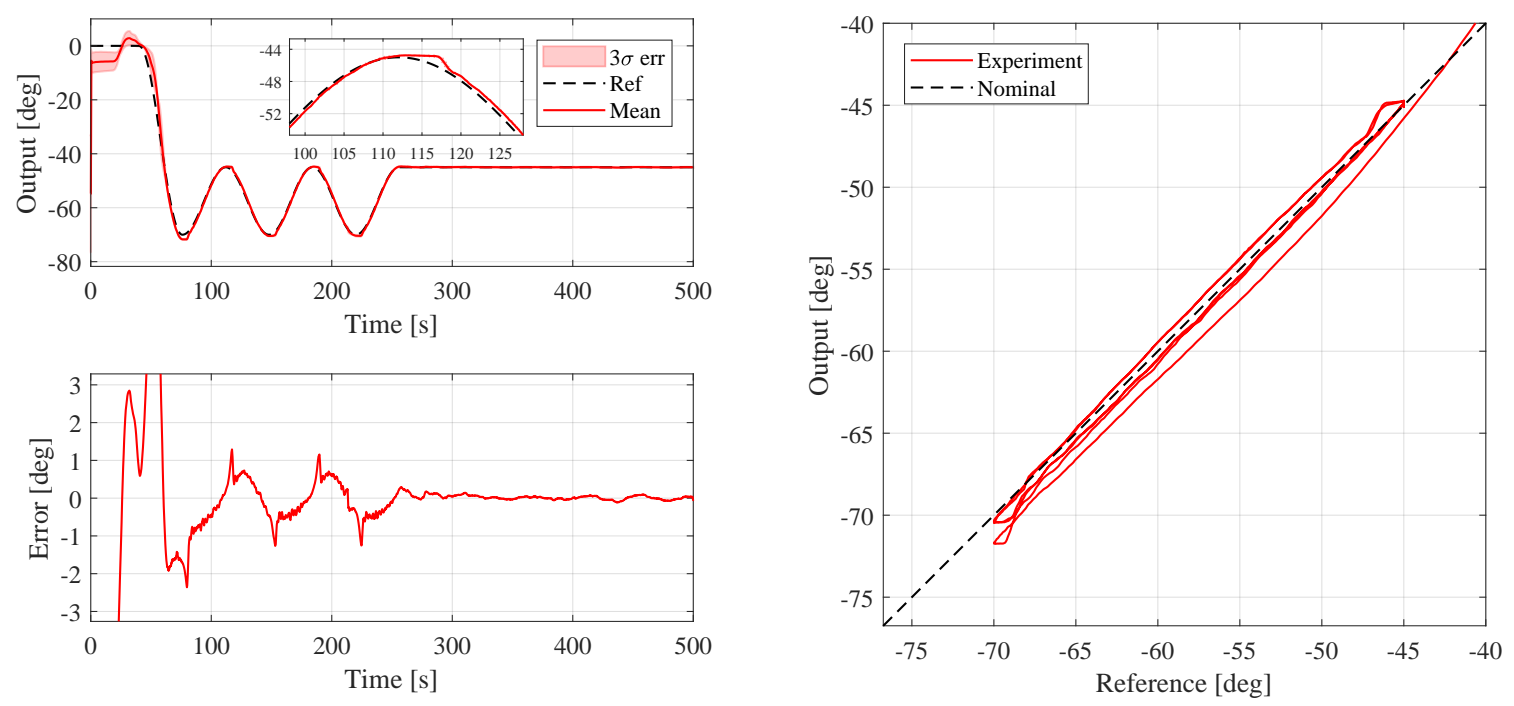

Figure 6.7: LP joint performance in straight arm configuration under AB control. Top: actual and desired proximal link angular trajectory, bottom: mean trajectory tracking error, and right: mean hysteresis loop of transient phase.

Overall, the AB controller showed excellent performance and a high degree of repeatability. Out of all the straight arm configuration cases, the AB controller demonstrates the lowest maximum error of roughly $1.2 \mathrm{deg}$. The phase delay in the system is greater than the expected performance of $\mathrm{AB}$ from the simulation, where the average delay in experiment was found to be 0.41 seconds. However, since it was established that the plant differs from the Bouc-Wen model, it is to be expected that the $\mathrm{AB}$ controller will have degraded performance in the presence of dynamics modelling errors. Aside from this, the $\mathrm{AB}$ controller demonstrates slightly more overshoot at the transient limits when compared to the PID controller, with excellent steady state performance. The bent arm case for the AB controlled system is shown 
in Fig. 6.8.
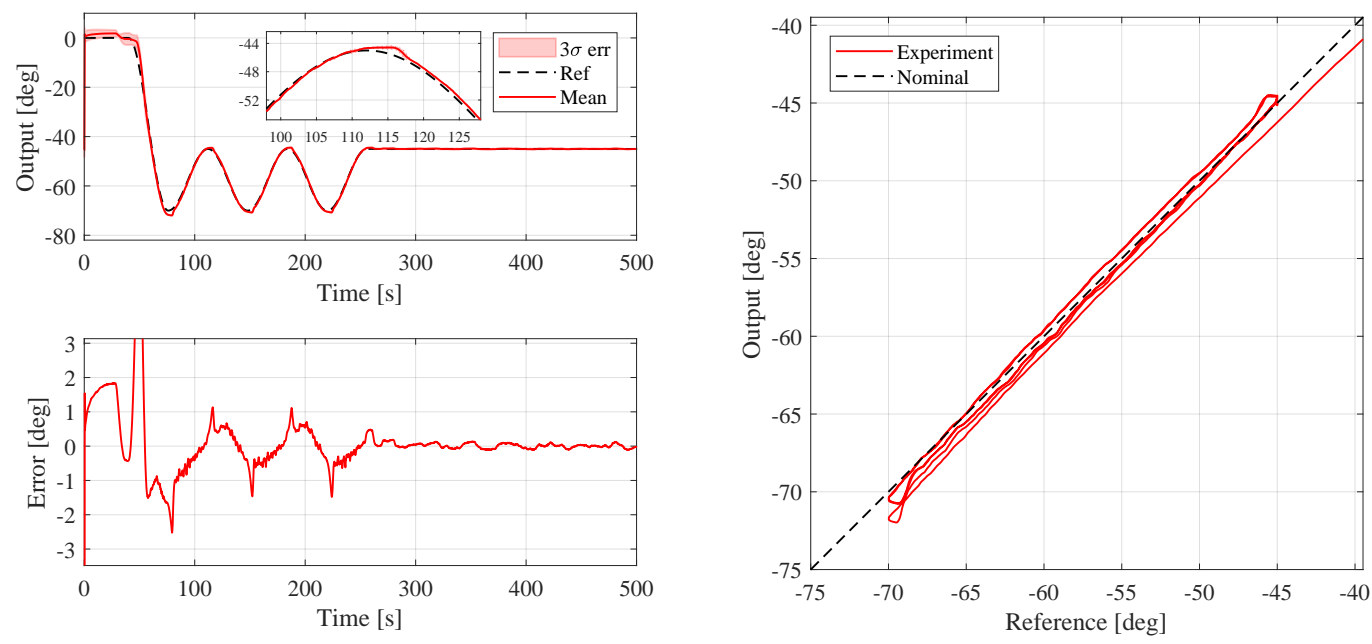

Figure 6.8: LP joint performance in bent arm configuration under AB control. Top: actual and desired proximal link angular trajectory, bottom: mean trajectory tracking error, and right: mean hysteresis loop of transient phase.

As was the case with all the other controllers, the AB performance in the bent arm case behaves similarly to its straight arm case with minor degradation. For instance, there now appear to be slight oscillations in the steady state region, which were not observed in the straight arm case. Furthermore, the peak error has increased to approximately $1.5 \mathrm{deg}$ after the learning phase; which was observed to occur over a longer period when in this arm configuration. With regards to the phase delay, there was nearly no difference from the straight arm case with the average delay being 0.40 seconds. Finally, the AB controller performance during the dynamic arm manoeuvre is captured in Fig. 6.9.

As with the previous control cases, the dead-zones have been eliminated, highfrequency oscillations have been introduced, and the repeatability has been degraded. With reference to the error plot, the magnitude of the error remained consistent with respect to the other arm configurations. Similarly, a comparable phase delay can be observed on the hysteresis plot, which suggests that this is an artefact of the experimental plant not matching the Bouc-Wen model exactly. Overall, the AB controller demonstrates a higher degree of consistency between its static and dynamic 
arm configurations when compared to the other controllers, implying that it may be the most reliable.
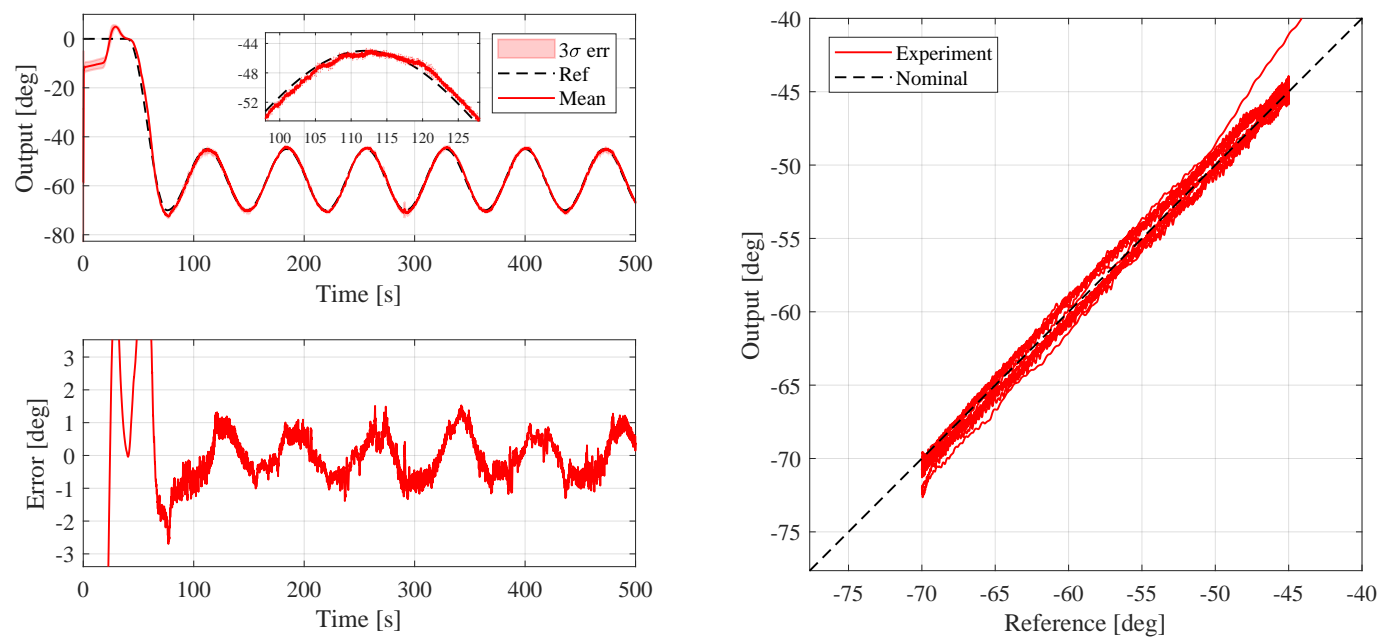

Figure 6.9: LP joint performance with moving arm under AB control. Top: actual and desired proximal link angular trajectory, bottom: mean trajectory tracking error, and right: mean hysteresis loop of transient phase. 


\section{5 $\mathcal{L}_{1}$ Control}

Finally, the performance of the $\mathcal{L}_{1}$ controlled LP joint is given here. Based on the tuning guidelines of the $\mathcal{L}_{1}$ controller in Section 5.4.1, the controller parameters chosen for the experiment were $\Gamma=2.5, k=1, t_{s}=2$, and $\psi=1 / 9$. For these gains, the $\mathcal{L}_{1}$ stability condition was met with $\|G(s)\|_{\mathcal{L}_{1}} L=0.9091$, as per Eq. (4.48). The performance of the $\mathcal{L}_{1}$ controlled LP joint is shown in Fig. 6.10 for the straight arm configuration. Note that there is an initially high error near the beginning of the experiment during the learning phase of the controller.
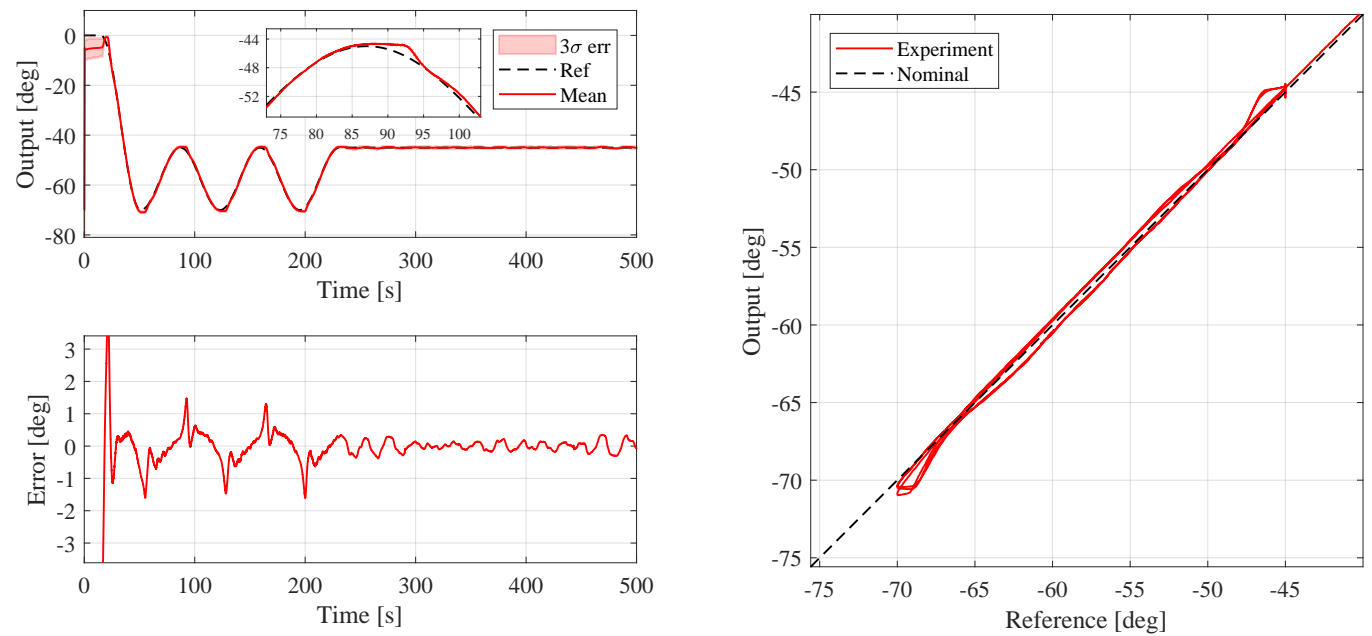

Figure 6.10: LP joint performance in straight arm configuration under $\mathcal{L}_{1}$ control. Top: actual and desired proximal link angular trajectory, bottom: mean trajectory tracking error, and right: mean hysteresis loop of transient phase.

Once again, the performance of the system was improved with the $3 \sigma$ error being negligible in terms of repeatability. An interesting thing to note is that the adaptive parameters for the $\mathcal{L}_{1}$ controller have a faster convergence time as compared to the $\mathrm{AB}$ controller; as is evident in the learning phase near the start of the experiment. Moreover, the average error was reduced, and phase lag in the system was found to be 0.32 seconds. This result is different than what was shown in simulation, where there was still a noticeable phase delay. However, note that the plant and controller gains for the experiment are different than what was performed in the simulation.

Despite the overall phase and error improvements, the hysteresis loop shows that 
there is a greater overshoot specifically at the limits of the transient wave compared to the PID and AB controlled cases (i.e. the output extends past the -70 deg mark). The overshoot is quite small, though it is worth noting since overshoot is undesirable in robotic applications. Furthermore, the $\mathcal{L}_{1}$ controller appears to give an oscillatory response in steady state, which was not exhibited in the previous control cases. It is difficult to determine the exact cause of this phenomenon since there are several gain parameters, though it is suspected that the filter could be optimized further to improve the results. Now, the tracking performance for the bent arm configuration in Fig. 6.11 will be investigated.
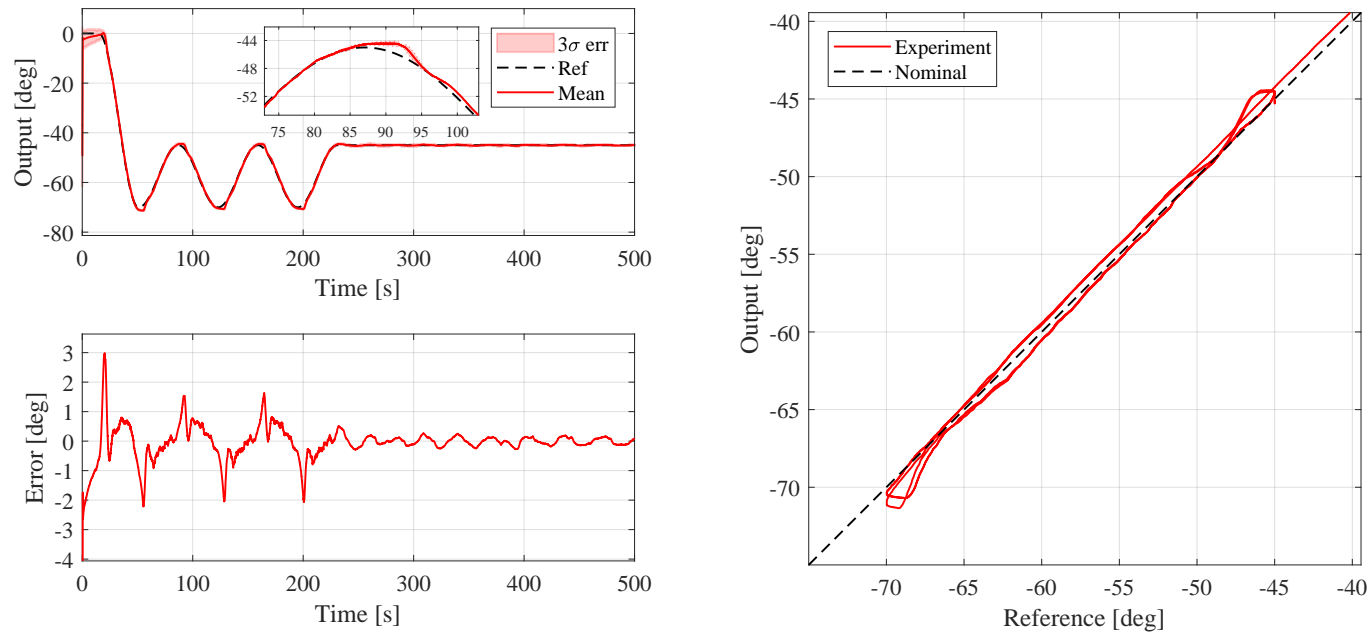

Figure 6.11: LP joint performance in bent arm configuration under $\mathcal{L}_{1}$ control. Top: actual and desired proximal link angular trajectory, bottom: mean trajectory tracking error, and right: mean hysteresis loop of transient phase.

When compared to the straight arm configuration, the bent arm case exhibited similar performance with only minor differences. In this case, the phase delay was slightly higher than the straight arm case with a value of 0.39 seconds; this is expected since there is more friction introduced to the system. Secondly, the overall error throughout the experiment was higher in general. That is to say, the peak error reached was roughly $2 \mathrm{deg}$, and the magnitude of the steady state oscillations has increased. What is most interesting about the $\mathcal{L}_{1}$ controller is its performance during the dynamic arm configuration, presented in Fig. 6.12. 
Overall, the average error is less than what was observed during the transient phases of the preceding arm configurations, where the error stays roughly under of $1.2 \mathrm{deg}$ throughout the experiment. This is likely due to the fact that the dead-zones have been reduced (as was observed in the previous dynamic arm cases), since deadzone performance was in fact the main source of error in the static arm cases. The phase delay in this case was imperceptible due to the high-frequency oscillations in the data. From the results obtained, it can be said that the $\mathcal{L}_{1}$ controller has the best performance under dynamic reference signals and arm configurations, while the AB controller performs best during a "hold" manoeuvre while the CSTM is in a static state. This implies that the $\mathcal{L}_{1}$ controller has the best performance when subjected to plant variations and unmodelled dynamics.
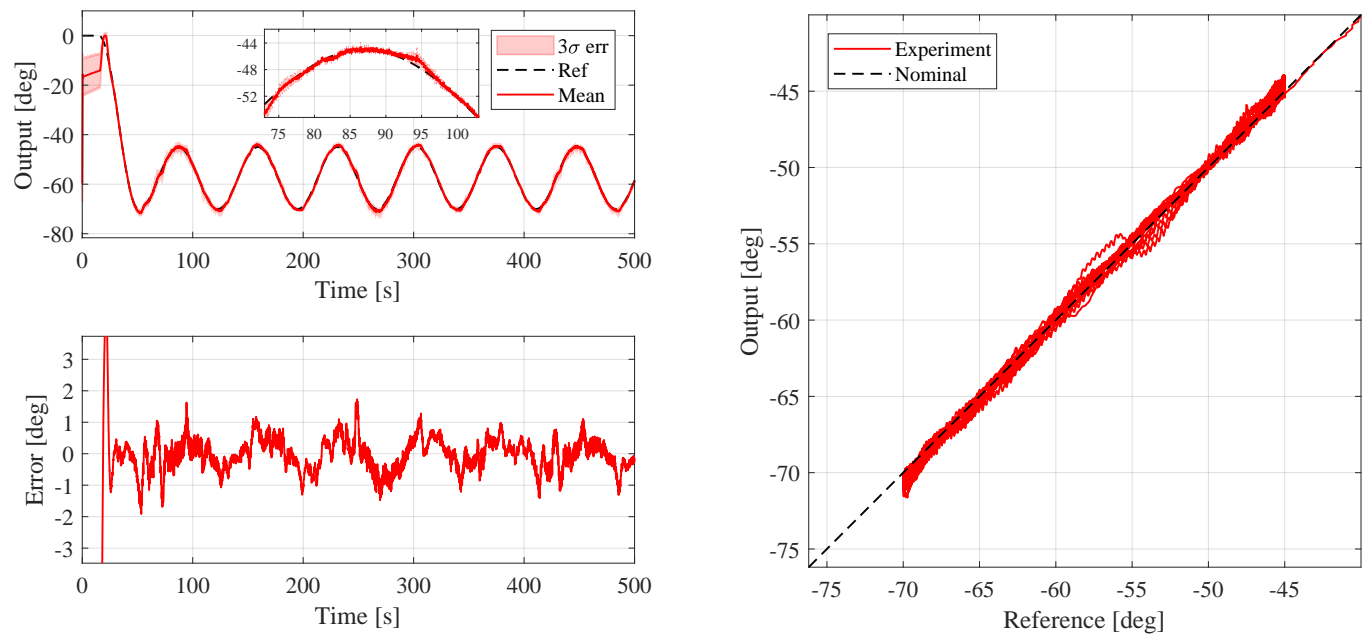

Figure 6.12: LP joint performance with moving arm under $\mathcal{L}_{1}$ control. Top: actual and desired proximal link angular trajectory, bottom: mean trajectory tracking error, and right: mean hysteresis loop of transient phase. 


\section{Chapter 7}

\section{Conclusion}

In this chapter, the motivation, research methodology, and results are briefly revisited to conclude the work performed in this thesis. Additionally, the contributions made to the field will be reviewed, along with a note on the applicability in a full-scale application and suggestions for improving upon this work are given.

\subsection{Thesis Summary}

As the space industry progresses, the number of artificial satellites and other objects will continue to populate Earth's orbit. In doing so, future and current missions are at risk of failure, potentially leading to a catastrophic outcome. This thesis proposed a universal capture mechanism to act as an EE on a servicing craft for the purposes of de-orbiting failed satellites, or for performing manipulation tasks in the context of servicing active spacecraft. This capture mechanism was designed as a robotic gripper driven by a CSTM with the capability of having a synthetic gecko adhesive contact interface on the links. This mechanism was developed in attempts to propose a solution to interface with a wide variety of target geometries.

Due to the complexity of the project, the scope was limited to the design, implementation, and control of the concept in a prototype environment for future testing of a full capture. First, an optimal joint design was determined to maximize joint torque based on the selected gripper concept and the proposed grip configurations. From these results, the actuation system was designed, followed by the full assembly and integration of the gripper onto the SPOT robotic manipulator. To control the gripper, a well performing indirect control algorithm was implemented, along with the direct $\mathcal{L}_{1}$ control law that has never before been applied to a CSTM. The performance of these controllers were compared against the baseline "no control" and "PID" cases in both the simulated and experimental environment. The simulations 
showed that the $\mathrm{AB}$ controller had a clear advantage over the $\mathcal{L}_{1}$ control law. This performance was expected since the model in which the $\mathrm{AB}$ controller was derived from was used to simulate the plant: implying that the system matches perfectly with what the controller is trying to estimate. This clear distinction, however, was not evident in the experimental tests. While the AB controller still demonstrated the best tracking performance in terms of minimizing the overall tracking, overshoot and steady state error, the $\mathcal{L}_{1}$ performed only slightly worse in these aspects while maintaining a better transient response by reducing the phase delay to nearly zero. From the results obtained, one can draw the conclusion that the $\mathcal{L}_{1}$ controller performs best in dynamic arm configurations, while the AB controller performs best during a "hold" manoeuvre while the CSTM is in a static state.

\subsection{Summary of Contributions}

Previously outlined in Section 1.5, there were several contributions to the field made by this work.

- A novel mechanism was proposed for the purposes of non-cooperative target capture, which includes a tendon-driven manipulator that has the capability of employing a synthetic gecko-adhesive as its contact interface.

- A multi-link gripper was developed as a prototype using optimal techniques for the SPOT platforms at the Carleton University SRCL.

- An indirect adaptive controller developed by Do et al. was applied to a full-scale multi-link robotic mechanism for the first time and validated experimentally.

- A direct $\mathcal{L}_{1}$ adaptive controller was applied to a CSTM for the first time and validated in simulations and experiments.

- A brief sensitivity study was presented as a means to qualitatively tune the AB and $\mathcal{L}_{1}$ controllers. 


\subsection{A Note on Practicality}

Given that the capture mechanism in this thesis was developed as a prototype, specifically for the SPOT facilities at the SRCL, it is important to make comments with regards to its applicability in a full-scale scenario. For instance, the design was made for a planar gripper to comply with the requirements of the testing facility. This decision, though necessary for the work in this thesis, is not a requirement for this mechanism. Several mechanisms exist for spatially articulated tendon-driven manipulators at a variety of scales; implying that this mechanism can be scaled up in both size and dimension to accommodate orbital target capture. In doing so, however, it is unpredictable how the complexity of the gripper would be affected in terms of the joint design, implementation, or control. Moreover, an in depth survey of potential capture interfaces on actual orbital targets would be required prior to designing the full system to maximize its utility over a broad range of targets.

It is also difficult to assess the practicality of such a technology (i.e. robotic gripper with gecko adhesive contact interfaces) prior to the full capture experiment being performed. It could likely be the case that this "mark 1.0" prototype is not the most optimal execution of the proposed hybridization since there are a number of factors that could be modified. For example, many of the design considerations were made based on cost and complexity given the time frame and cost limitations of the project; including the elimination of pulleys or encoders directly at the joints to simplify assembly at that scale, utilizing springs to return the gripper to neutral position at the expense of joint stiffness, and relying exclusively on state feedback from the joints when in fact force-feedback may be required for a full capture.

Furthermore, there are a plethora of gecko adhesives that could be employed to the links of the gripper. Since this is largely an unknown factor at this phase in the project's development, there may still be unknown factors that render its hybridization into the gripper ineffective, or infeasible. Overall, since this technology is in the infancy stage of its development, the applicability of its use in practice is left as an open ended problem.

With regards to the implementation of the control laws to a CSTM, however, it was shown that both solutions have their advantages and limitations when implemented 
into a physical system. When introduced to a different gripper that utilizes a CSTM, there is a possibility that there is a clear distinction for which controller performs best. Additionally, the performances of these controllers may in fact be sub-optimal since the tuning performed was based on qualitative tuning methods to the best of the author's ability. Thus, it could be likely that there is a performance limitation in these control laws that make the CSTM an infeasible option for orbital capture, however, this would need to be confirmed with a full-scale test on high quality equipment that would be expected to operate in the real application.

\subsection{Future Work}

As alluded to by the previous section, there are many aspects in which this project could be further improved by future contributions:

1. Since this work was only concerned with the design, implementation, and position control of the gripper, it is an obvious extension of this work that a full capture experiment be conducted. Prior to the full capture, the selection and procurement of an appropriate gecko adhesive would be required. Further more, a suitable capture strategy would have to be developed in order to experimentally validate the prototype within the SRCL facilities.

2. In context of performing a capture, a potential capture strategy would be to employ an impedance based control law to minimize contact overshoot caused by the capture manoeuvre. This would ultimately require force feedback of the EE which could be achieved via force-moment sensor at the wrist, strain-gauge force feedback of the individual links, or by developing a non-linear disturbance observer for the gripper.

3. In the event that the PhaseSpace measurement system is not available (i.e. in practice) the inclusion of high-resolution encoders directly at the joints for the state feedback would be a required substitute.

4. There are several types of $\mathcal{L}_{1}$ control architectures that exist. A possible extension would be to investigate their performance upon the closed loop system 
and verify them experimentally.

5. The development of an optimal tuning strategy (such as parameter estimation via hardware-in-the-loop) could be employed prior to the experiment to determine the set of optimal gains for the controller of interest. 


\section{Bibliography}

[1] Kelso, T. S., "CelesTrak SATCAT Boxscore Database," https://www.celestrak.com/satcat/search.php, March 2018, [Accessed: 15 Mar 2018].

[2] Kessler, D. J. and Cour-Palais, B. G., "Collision frequency of artificial satellites: The creation of a debris belt," Journal of Geophysical Research: Space Physics, Vol. 83, No. A6, 1978, pp. 2637-2646.

[3] Liou, J.-C. and Johnson, N. L., "Instability of the present LEO satellite populations," Advances in Space Research, Vol. 41, No. 7, 2008, pp. 1046-1053.

[4] Schaub, H., Jasper, L. E., Anderson, P. V., and McKnight, D. S., "Cost and risk assessment for spacecraft operation decisions caused by the space debris environment," Acta Astronautica, Vol. 113, 2015, pp. 66-79.

[5] Kessler, D. J., Johnson, N. L., Liou, J., and Matney, M., "The kessler syndrome: implications to future space operations," Advances in the Astronautical Sciences, Vol. 137, No. 8, 2010, pp. 2010.

[6] Shan, M., Guo, J., and Gill, E., "Review and comparison of active space debris capturing and removal methods," Progress in Aerospace Sciences, Vol. 80, 2016, pp. $18-32$.

[7] Wormnes, K., Le Letty, R., Summerer, L., Schonenborg, R., Dubois-Matra, O., Luraschi, E., Cropp, A., Krag, H., and Delaval, J., "ESA technologies for space debris remediation," 6th European Conference on Space Debris, Vol. 1, ESA Communications ESTEC, Noordwijk, The Netherlands, 2013, pp. 1-8.

[8] Pardini, C., Hanada, T., and Krisko, P. H., "Benefits and risks of using electrodynamic tethers to de-orbit spacecraft," Acta Astronautica, Vol. 64, No. 5-6, 2009, pp. 571-588.

[9] Ziegler, S. W. and Cartmell, M. P., "Using motorized tethers for payload orbital transfer," Journal of Spacecraft and Rockets, Vol. 38, No. 6, 2001, pp. 904-913.

[10] Hovell, K. and Ulrich, S., "Attitude stabilization of an uncooperative spacecraft in an orbital environment using visco-elastic tethers," AIAA Guidance, Navigation, and Control Conference, 2016, p. 0641.

[11] Williams, P., "In-plane payload capture with an elastic tether," Journal of guidance, control, and dynamics, Vol. 29, No. 4, 2006, pp. 810-821. 
[12] Carta, R. and Benvenuto, R., "Implementation of a net device test bed for space debris active removal feasibility demonstration," 2012.

[13] Dudziak, R., Tuttle, S., and Barraclough, S., "Harpoon technology development for the active removal of space debris," Advances in Space Research, Vol. 56, No. 3, 2015, pp. 509-527.

[14] Saunders, C., Chiesa, A., Forshaw, J., Parreira, B., and Biesbroek, R., "Results of a system feasibility study on a heavy active debris removal mission," 2014.

[15] Oda, M., Kibe, K., and Yamagata, F., "ETS-VII, space robot in-orbit experiment satellite," Proceedings of IEEE international conference on robotics and automation, Vol. 1, IEEE, 1996, pp. 739-744.

[16] Jorgensen, G. and Bains, E., "SRMS history, evolution and lessons learned," AIAA SPACE 2011 Conference \& Exposition, 2011, p. 7277.

[17] Ticker, R. L., Cepollina, F., and Reed, B. B., "NASAs in-space robotic servicing," AIAA SPACE 2015 Conference and Exposition, 2015, p. 4644.

[18] Romano, M., Friedman, D. A., and Shay, T. J., "Laboratory experimentation of autonomous spacecraft approach and docking to a collaborative target," Journal of Spacecraft and Rockets, Vol. 44, No. 1, 2007, pp. 164-173.

[19] Pinson, R., Howard, R., and Heaton, A., "Orbital express advanced video guidance sensor: ground testing, flight results and comparisons," AIAA guidance, navigation and control conference and exhibit, 2008, p. 7318.

[20] Henshaw, C. G., "The darpa phoenix spacecraft servicing program: Overview and plans for risk reduction," International Symposium on Artificial Intelligence, Robotics and Automation in Space (i-SAIRAS), 2014.

[21] Debus, T. and Dougherty, S., "Overview and performance of the front-end robotics enabling near-term demonstration (FREND) robotic arm," AIAA Infotech@Aerospace Conference and AIAA Unmanned... Unlimited Conference, p. 1870.

[22] Wieser, M., Richard, H., Hausmann, G., Meyer, J.-C., Jaekel, S., Lavagna, M., and Biesbroek, R., "e. Deorbit Mission: OHB Debris Removal Concepts," 2015.

[23] Ratti, J., "Launch Adapter Ring (LAR) Capture Tool: Enabling Space Robotic Servicing," Proceedings of International Conference on Robotics and Automation, 2015.

[24] Richard, M., Kronig, L., Belloni, F., Rossi, S., Gass, V., Paccolat, C., Thiran, J., Araomi, S., Gavrilovich, I., and Shea, H., "Uncooperative rendezvous and docking for MicroSats," 6th international conference on recent advances in space technologies, 2013. 
[25] Trentlage, C. and Stoll, E., "The Applicability of Gecko Adhesives in a Docking Mechanism for Active Debris Removal Missions," Symposium on Advanced Space Technologies in Robotics and Automation, Noordwijk, Netherlands, 2015.

[26] Jiang, H., Hawkes, E. W., Arutyunov, V., Tims, J., Fuller, C., King, J. P., Seubert, C., Chang, H. L., Parness, A., and Cutkosky, M. R., "Scaling controllable adhesives to grapple floating objects in space," 2015 IEEE International Conference on Robotics and Automation (ICRA), IEEE, 2015, pp. 2828-2835.

[27] Parness, A. and Soto, D., "A microfabricated wedge-shaped adhesive array displaying gecko-like dynamic adhesion, directionality and long lifetime," Journal of the Royal Society, 2009, Available Online, http://rsif.royalsocietypublishing.org/content/early/2009/03/13/rsif.2009.0048.short [Accessed: May 2017].

[28] Smith, A. M. and Callow, J. A., editors, Biological Adhesives, chap. 12: Properties, Principles, and Parameters of the Gecko Adhesive System, Springer, 2006, pp. 236-256.

[29] Soto, D., Hill, G., Parness, A., Esparza, N., Cutkosky, M., and Kenny, T., "Effect of fibril shape on adhesive properties," Applied Physics Letters, Vol. 97, No. 5, 2010, pp. 053701.

[30] Krahn, J., Liu, Y., Sadeghi, A., and Menon, C., "A tailless timing belt climbing platform utilizing dry adhesives with mushroomcaps," Smart Materials and Structures, Vol. 20, No. 11, 2011, pp. 115021.

[31] Ozawa, R., Kobayashi, H., and Hashirii, K., "Analysis, classification, and design of tendon-driven mechanisms," IEEE transactions on robotics, Vol. 30, No. 2, 2014, pp. 396-410.

[32] Ma, S., Hirose, S., and Yoshinada, H., "Design and experiments for a coupled tendon-driven manipulator," IEEE Control Systems Magazine, Vol. 13, No. 1, 1993, pp. 30-36.

[33] Le, H. M., Do, T. N., and Phee, S. J., "A survey on actuators-driven surgical robots," Sensors and Actuators A: Physical, Vol. 247, 2016, pp. 323-354.

[34] Tsai, L.-W., "Design of tendon-driven manipulators," Journal of mechanical design, Vol. 117, No. B, 1995, pp. 80-86.

[35] Kobayashi, H., Hyodo, K., and Ogane, D., "On tendon-driven robotic mechanisms with redundant tendons," The International Journal of Robotics Research, Vol. 17, No. 5, 1998, pp. 561-571. 
[36] Fuxiang, T. and Xingsong, W., "The design of a tendon-sheath-driven robot," 2008 15th International Conference on Mechatronics and Machine Vision in Practice, IEEE, 2008, pp. 280-284.

[37] Lin, C., Xingsong, W., and Fuxiang, T., "Tendon-sheath actuated robots and transmission system," Mechatronics and Automation, 2009. ICMA 2009. International Conference on, IEEE, 2009, pp. 3173-3178.

[38] Kaneko, M., Yamashita, T., and Tanie, K., "Basic considerations on transmission characteristics for tendon drive robots," Advanced Robotics, 1991.'Robots in Unstructured Environments', 91 ICAR., Fifth International Conference on, IEEE, 1991, pp. 827-832.

[39] Kaneko, M., Wada, M., Maekawa, H., and Tanie, K., "A new consideration on tendon-tension control system of robot hands," Proceedings. 1991 IEEE International Conference on Robotics and Automation, IEEE, 1991, pp. 1028-1033.

[40] Palli, G. and Melchiorri, C., "Model and control of tendon-sheath transmission systems," Proceedings 2006 IEEE International Conference on Robotics and Automation, 2006. ICRA 2006., IEEE, 2006, pp. 988-993.

[41] Palli, G., Borghesan, G., and Melchiorri, C., "Tendon-based transmission systems for robotic devices: Models and control algorithms," Robotics and Automation, 2009. ICRA'09. IEEE International Conference on, IEEE, 2009, pp. 4063-4068.

[42] Palli, G. and Melchiorri, C., "Optimal control of tendon-sheath transmission systems," IFAC Proceedings Volumes, Vol. 39, No. 15, 2006, pp. 73-78.

[43] Agrawal, V., Peine, W. J., Yao, B., and Choi, S., "Control of cable actuated devices using smooth backlash inverse," Robotics and Automation (ICRA), 2010 IEEE International Conference on, IEEE, 2010, pp. 1074-1079.

[44] Do, T., Tjahjowidodo, T., Lau, M., Yamamoto, T., and Phee, S., "Hysteresis modeling and position control of tendon-sheath mechanism in flexible endoscopic systems," Mechatronics, Vol. 24, No. 1, 2014, pp. 12-22.

[45] Do, T. N., Tjahjowidodo, T., Lau, M. W. S., and Phee, S. J., "Enhanced performances for cable-driven flexible robotic systems with asymmetric backlash profile," Technologies for Practical Robot Applications (TePRA), 2015 IEEE International Conference on, IEEE, 2015, pp. 1-6.

[46] Do, T. N., Tjahjowidodo, T., Lau, M. W. S., and Phee, S. J., "Position control of asymmetric nonlinearities for a cable-conduit mechanism," IEEE Transactions on Automation Science and Engineering, Vol. 14, No. 3, 2017, pp. 1515-1523. 
[47] Kaufman, H., Barkana, I., and Sobel, K., Direct adaptive control algorithms: theory and applications, Springer Science \& Business Media, 2012.

[48] Palli, G., Model and control of tendon actuated robots, Ph.D. thesis, alma, 2007.

[49] MathWorks, "Choosing the Algorithm," https://www.mathworks.com/help/optim/ug/choosin the-algorithm.html, 2018, [Accessed: 2 May 2018].

[50] Wright, S. and Nocedal, J., Numerical Optimization, chap. 18, Springer Science, 1999 .

[51] Taylor, J. C. and Stanton, J. F., "Friction coefficients for stainless steel (PTFE) teflon bearings," Tech. rep., 2010.

[52] Hovell, K., Detumbling Space Debris Using Tethers, Master's thesis, Carleton University, Ottawa, Ontario, 2017.

[53] Crain, A., Optimal Trajectory Planning and Compliant Spacecraft Capture Using a Space Robot, Master's thesis, Carleton University, Ottawa, Ontario, 2018.

[54] SRCL, C. U., "Facilities," https://carleton.ca/spacecraft/about/, 2019, [Accessed: 2 Mar 2019].

[55] Song, J. and Der Kiureghian, A., "Generalized Bouc-Wen model for highly asymmetric hysteresis," Journal of engineering mechanics, Vol. 132, No. 6, 2006, pp. 610-618.

[56] Zhang, J., Yang, Q., and Zhou, C., "L1 Adaptive Control Design for Hysteresis Compensation within Piezoelectric Actuators," IFAC Proceedings Volumes, Vol. 47, No. 3, 2014, pp. 2691-2696.

[57] Fan, X. and Smith, R. C., "L1 adaptive control of hysteresis in smart materials," Modeling, Signal Processing, and Control for Smart Structures 2008, Vol. 6926, International Society for Optics and Photonics, 2008, p. 69260G.

[58] Zou, X., Cao, C., and Hovakimyan, N., "L1 adaptive controller for systems with hysteresis uncertainties," Proceedings of the 2010 American Control Conference, IEEE, 2010, pp. 6662-6667.

[59] Hovakimyan, N. and Cao, C., L1 adaptive control theory: guaranteed robustness with fast adaptation, Vol. 21, SIAM-Society for Industrial and Applied Mathematics, 2010.

[60] Lord, W. and Hwang, J. H., "DC servomotors-modeling and parameter determination," IEEE Transactions on Industry Applications, , No. 3, 1977, pp. 234-243.

[61] Jafarnejadsani, H., Sun, D., Lee, H., and Hovakimyan, N., "Optimized L1 Adaptive Controller for Trajectory Tracking of an Indoor Quadrotor," Journal of Guidance, Control, and Dynamics, Vol. 40, No. 6, 2017, pp. 1415-1427. 


\section{Appendix A}

\section{Working Drawings}

This appendix presents the assembly and part drawings of the gripper in Section A.1 and the actuator bank in Section A.2.

\section{A.1 Gripper Assembly and Parts}

The drawings for the gripper will be presented on the following page. 


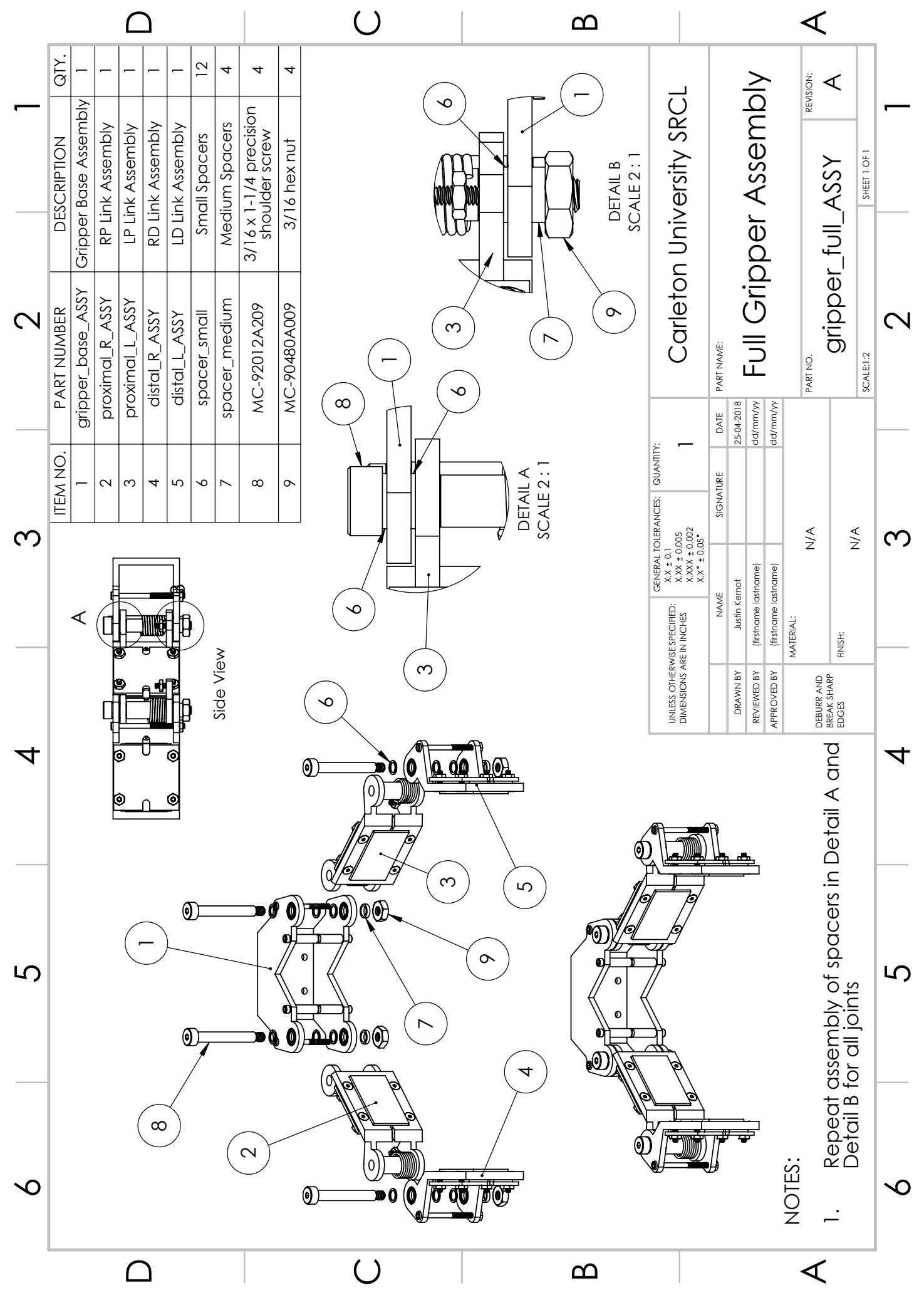




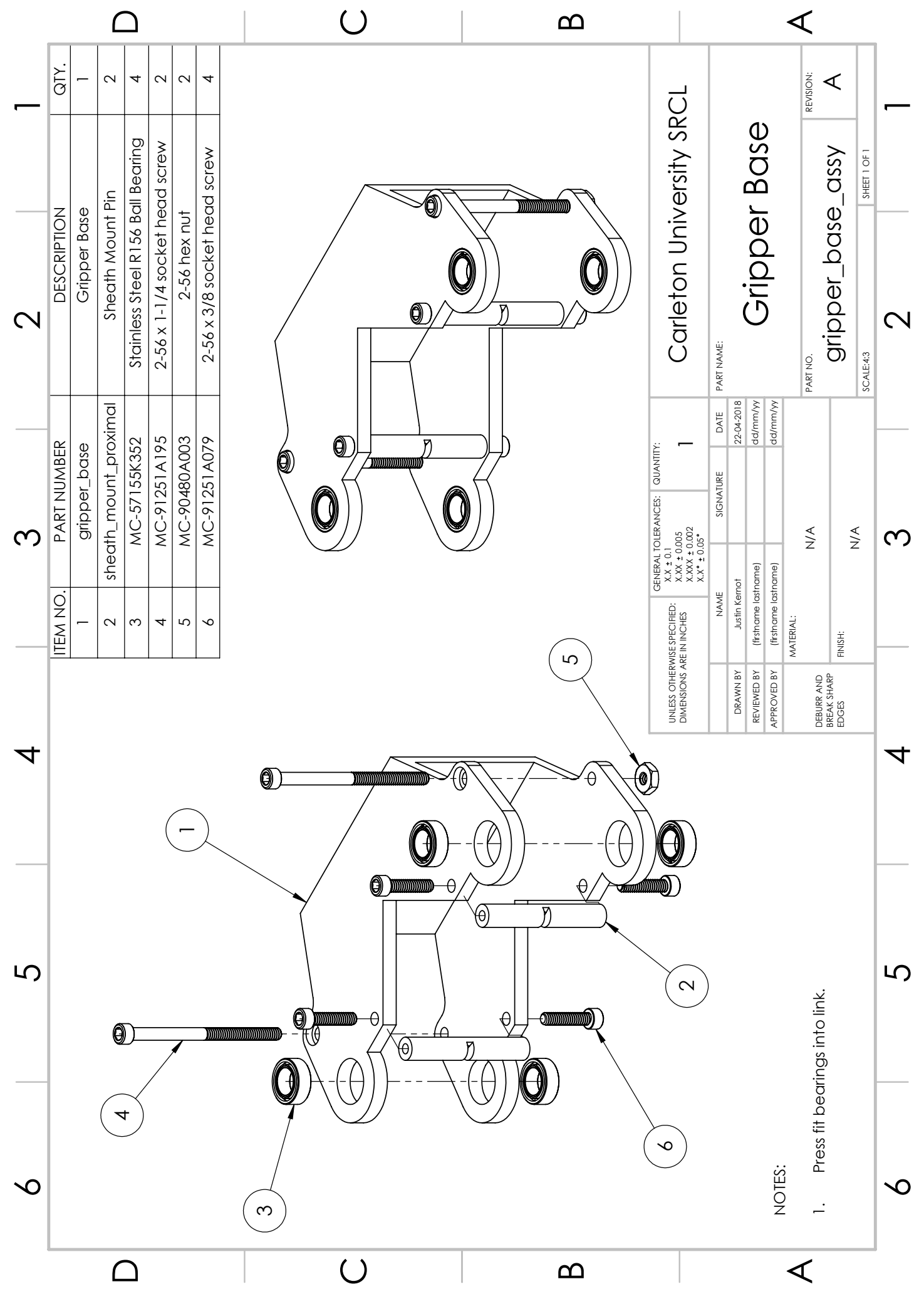




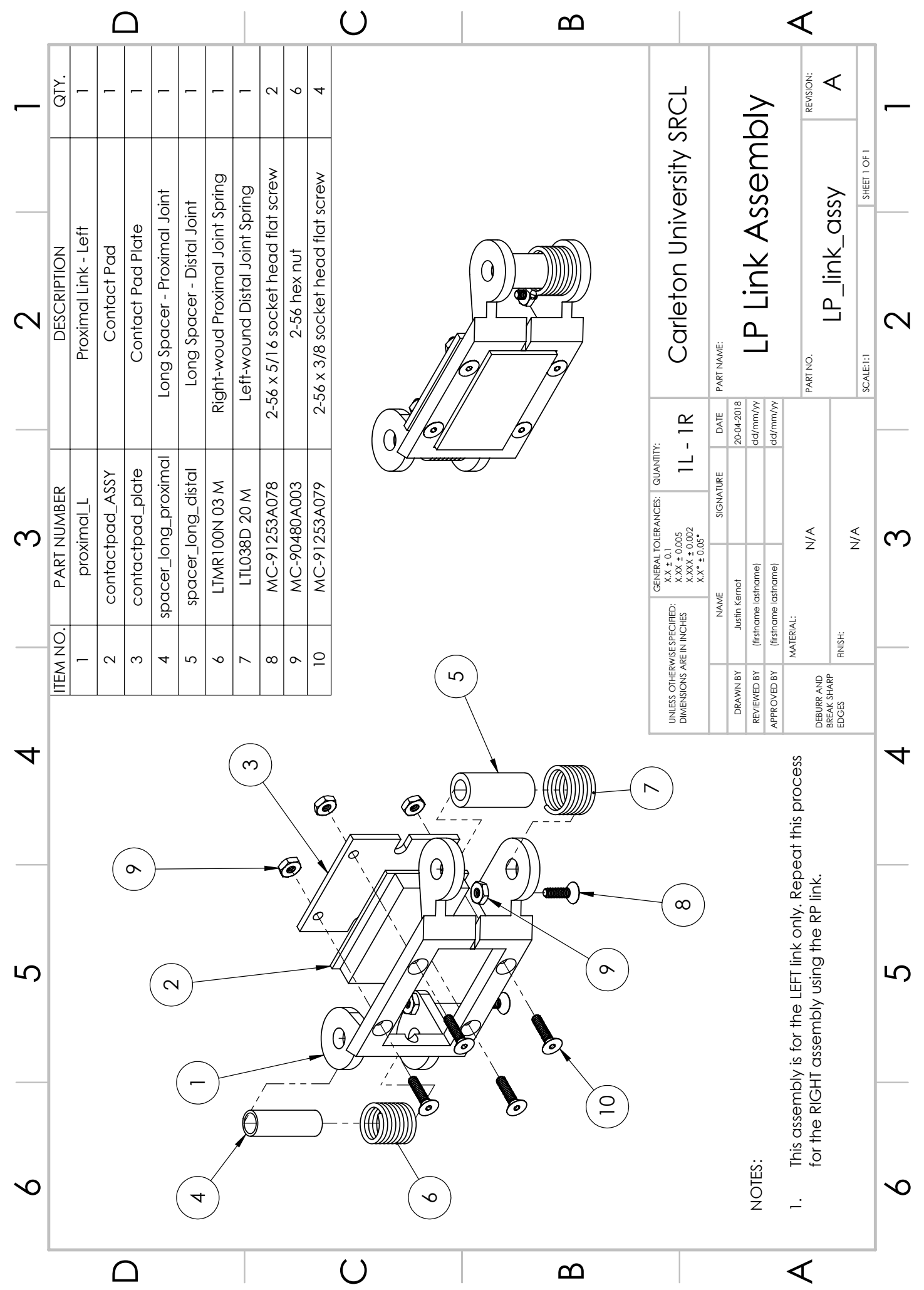




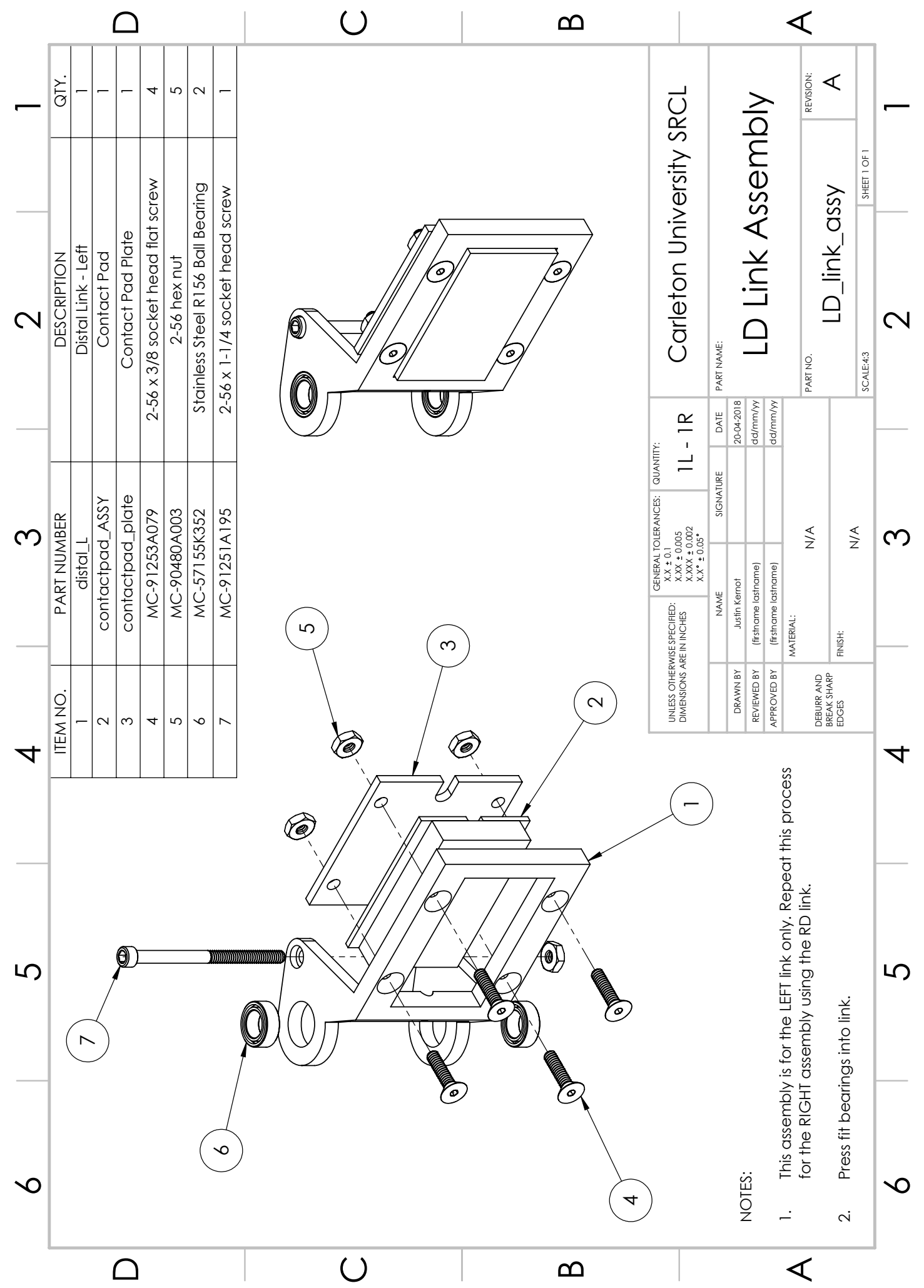




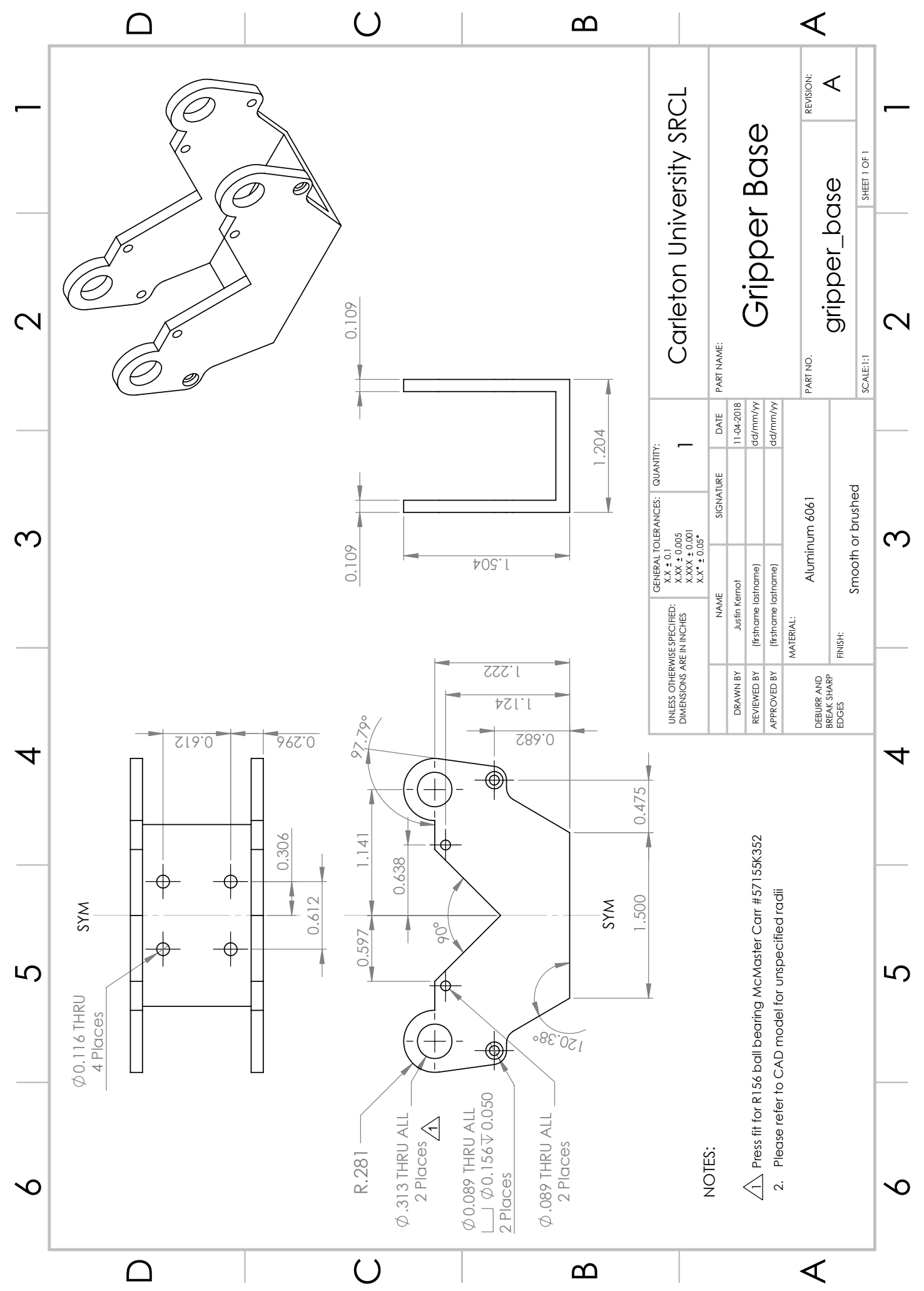




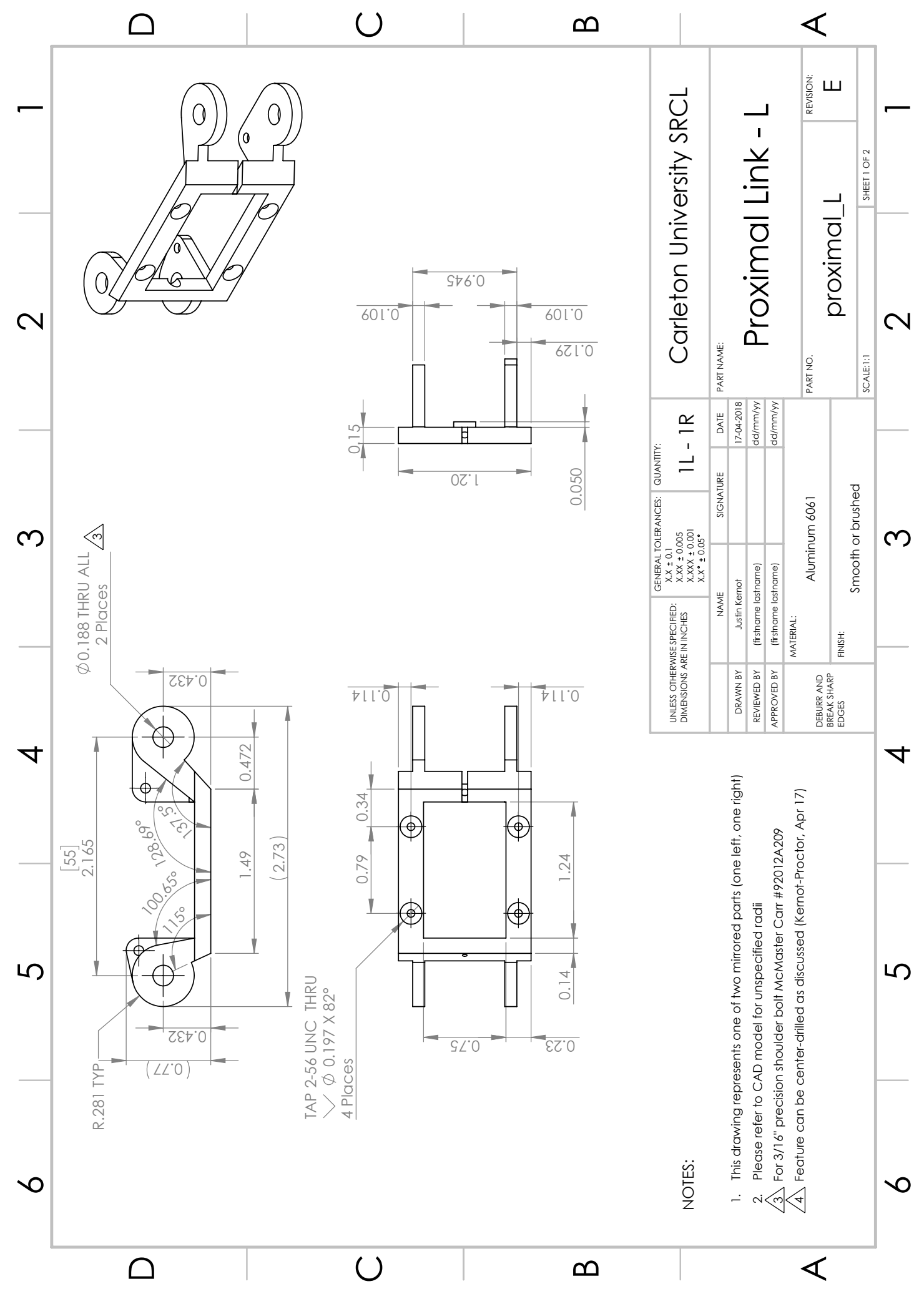




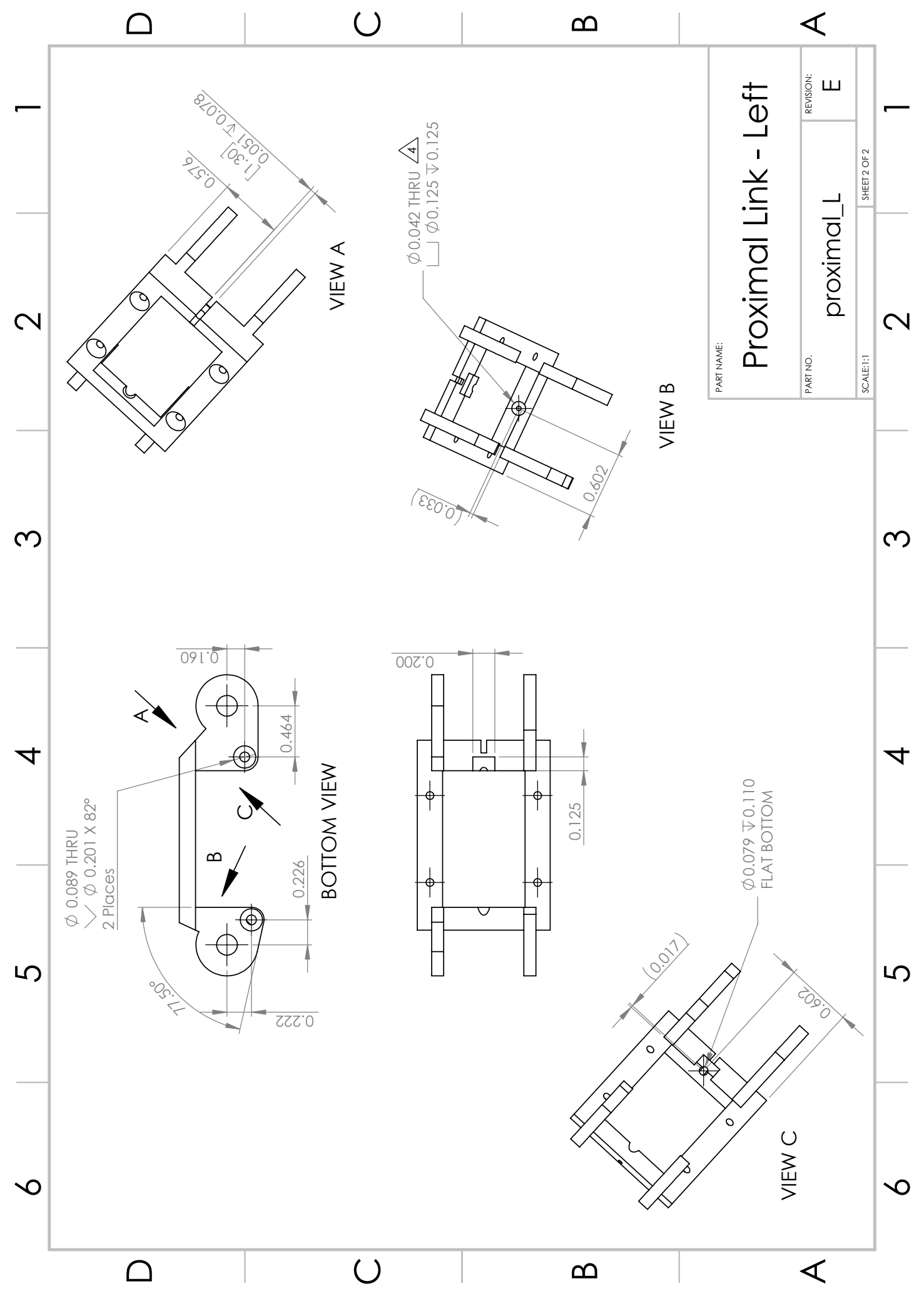




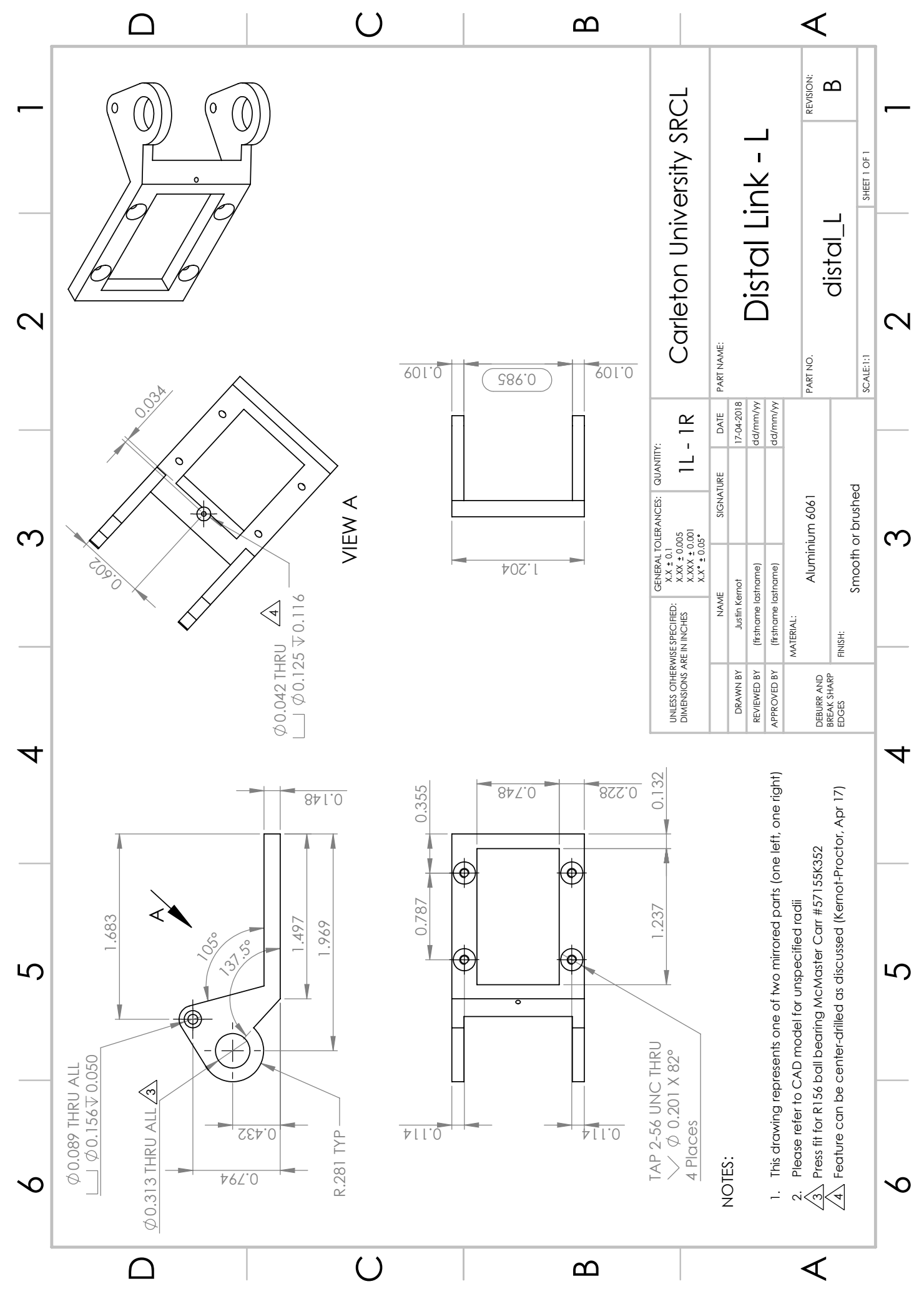




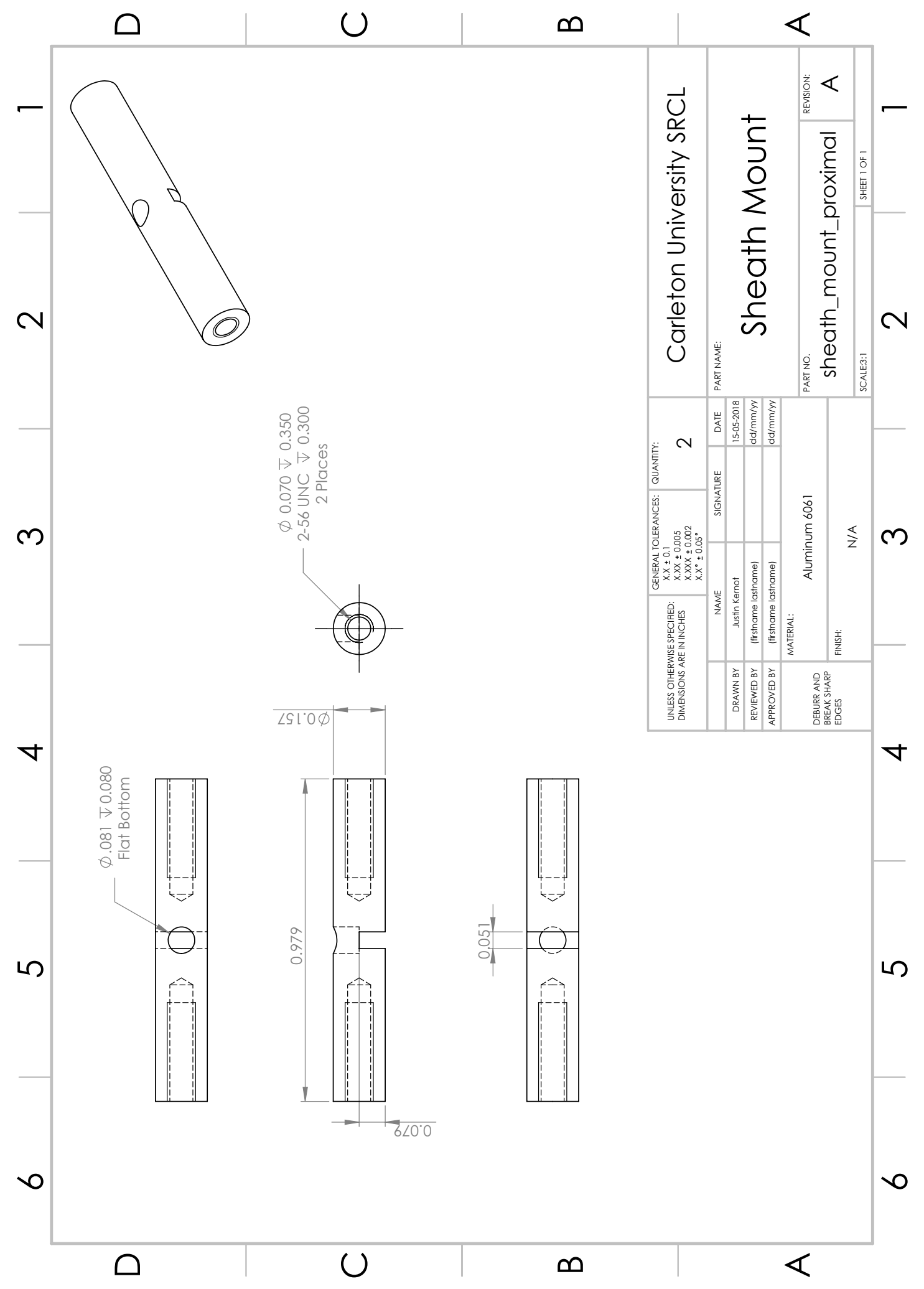




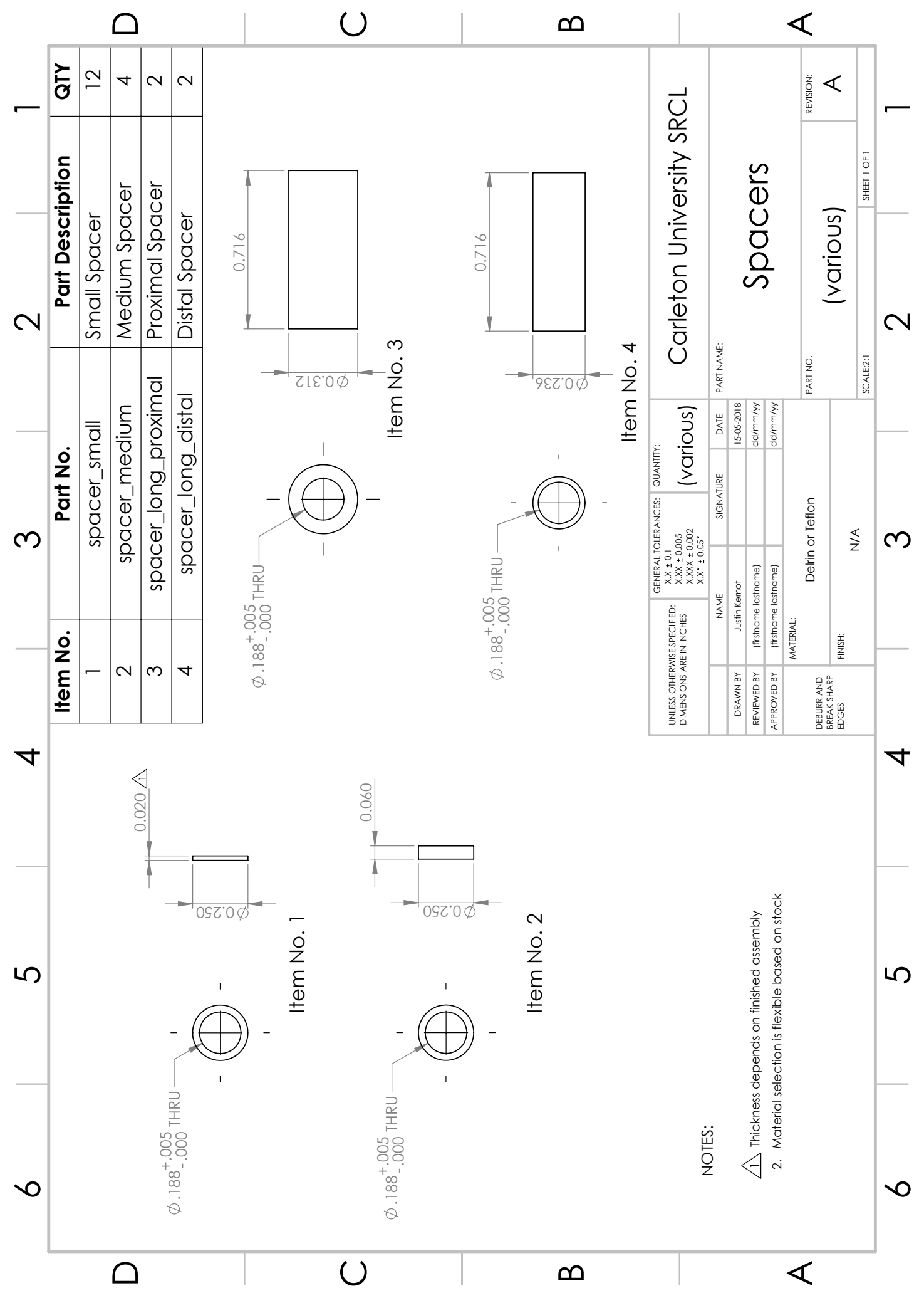




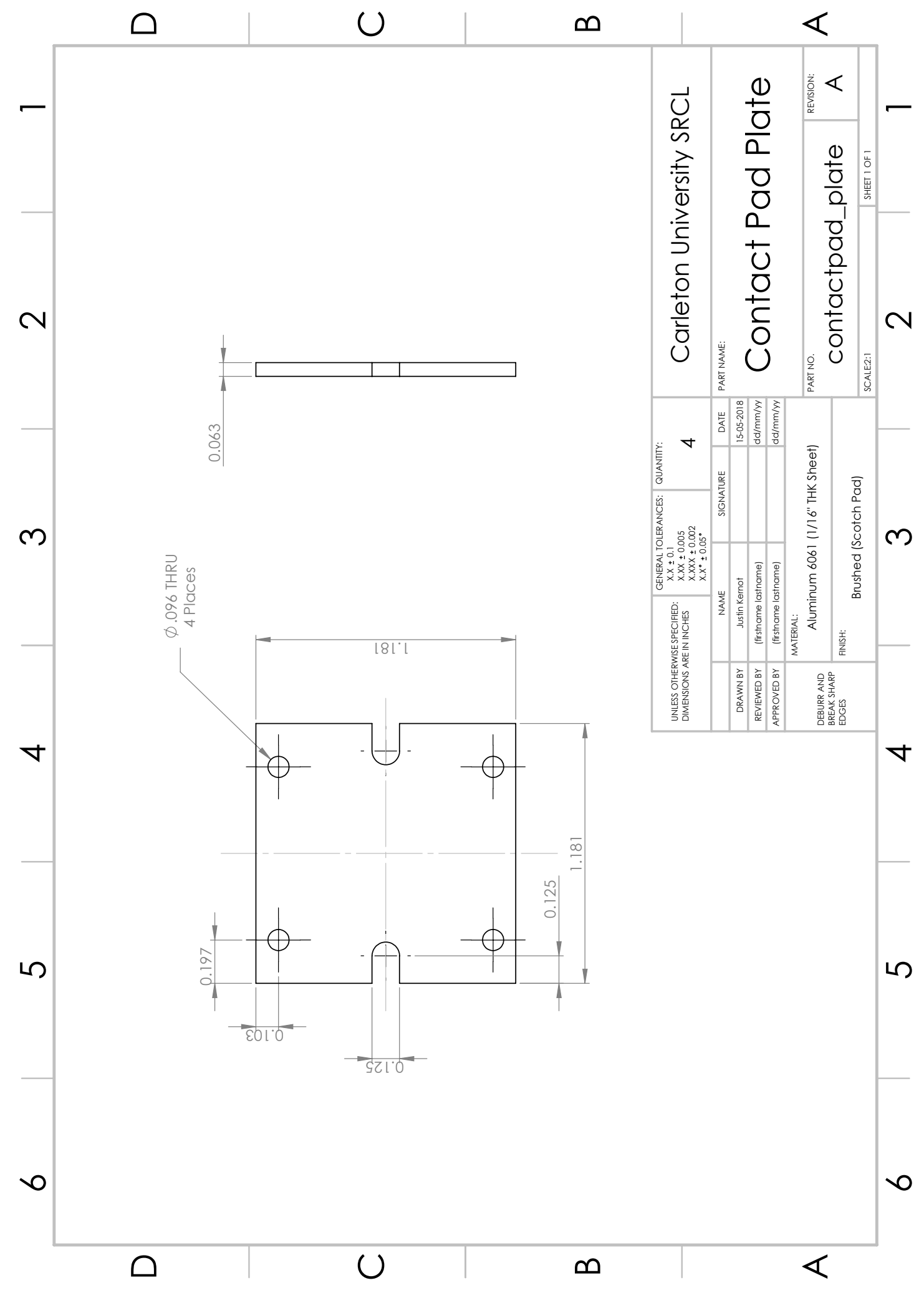




\section{A.2 Actuator Bank Assembly and Parts}

The drawings for the actuator bank will be presented on the following page. 


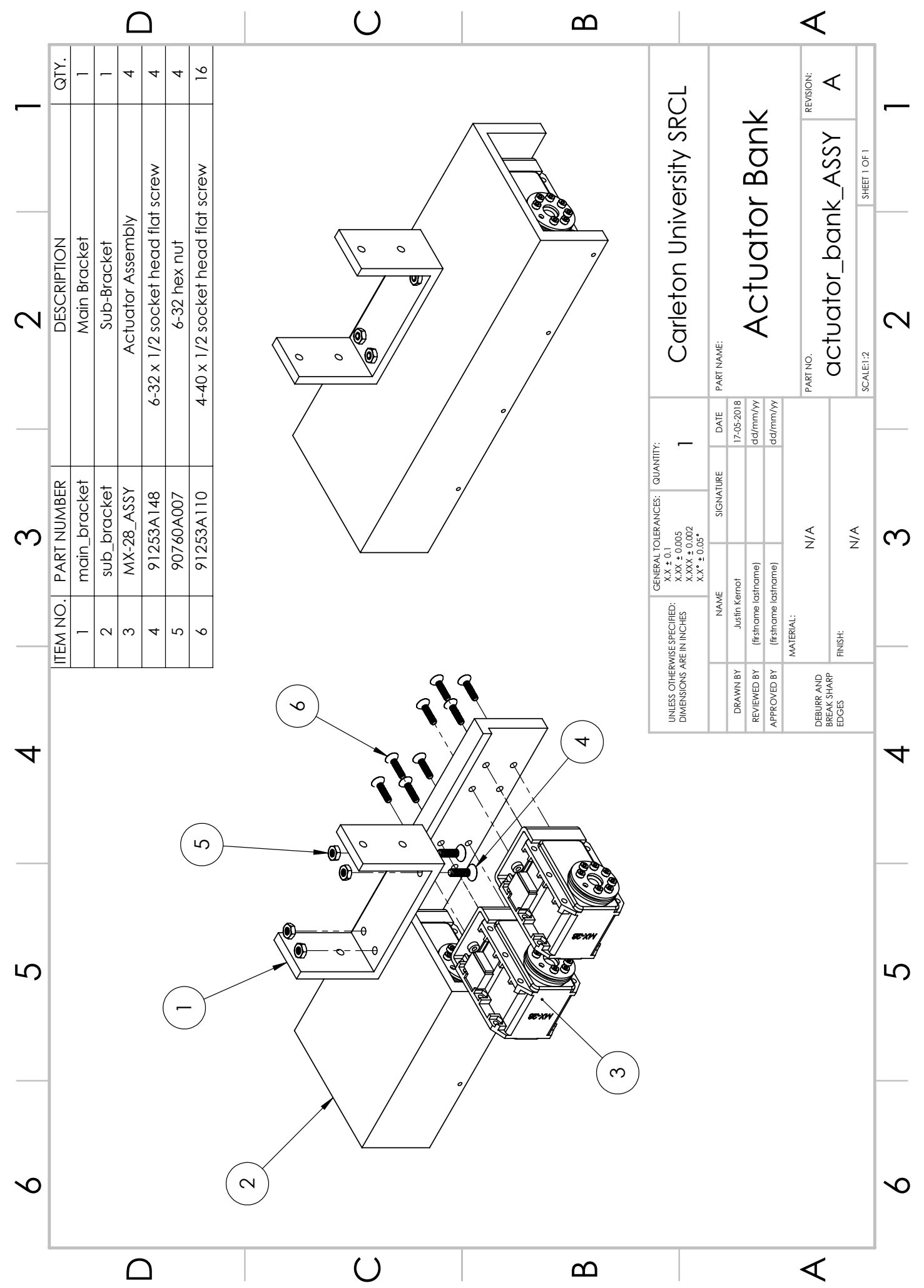




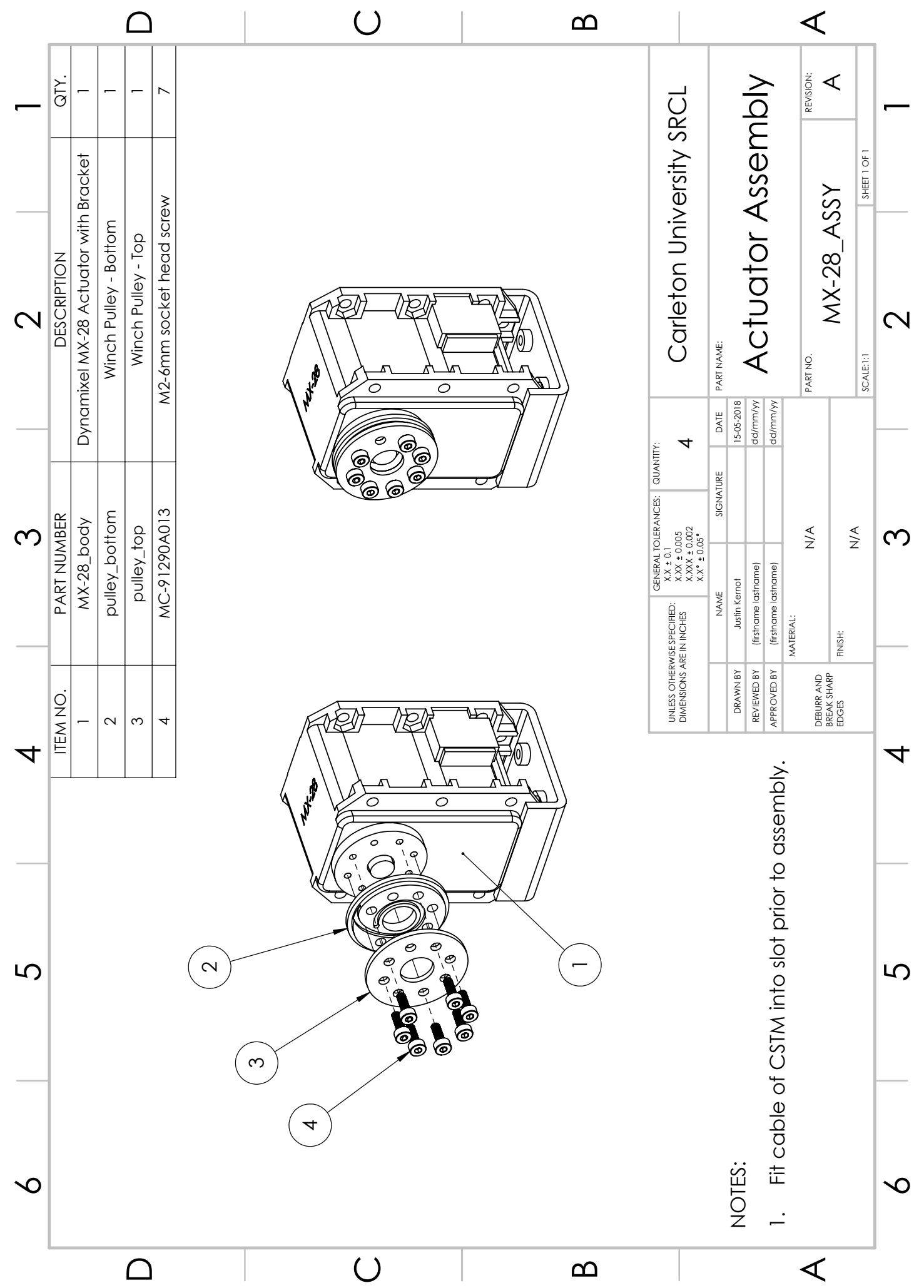




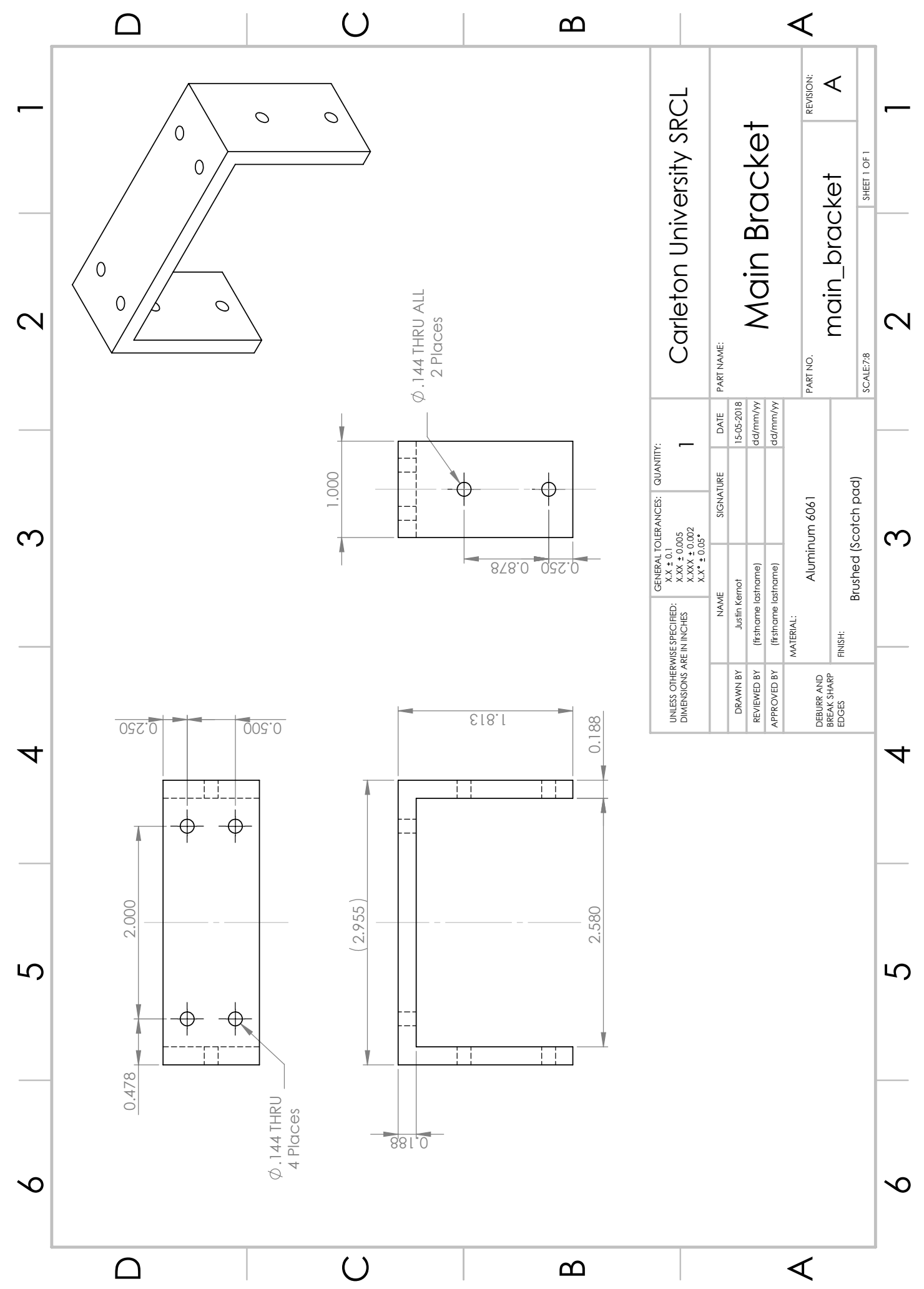




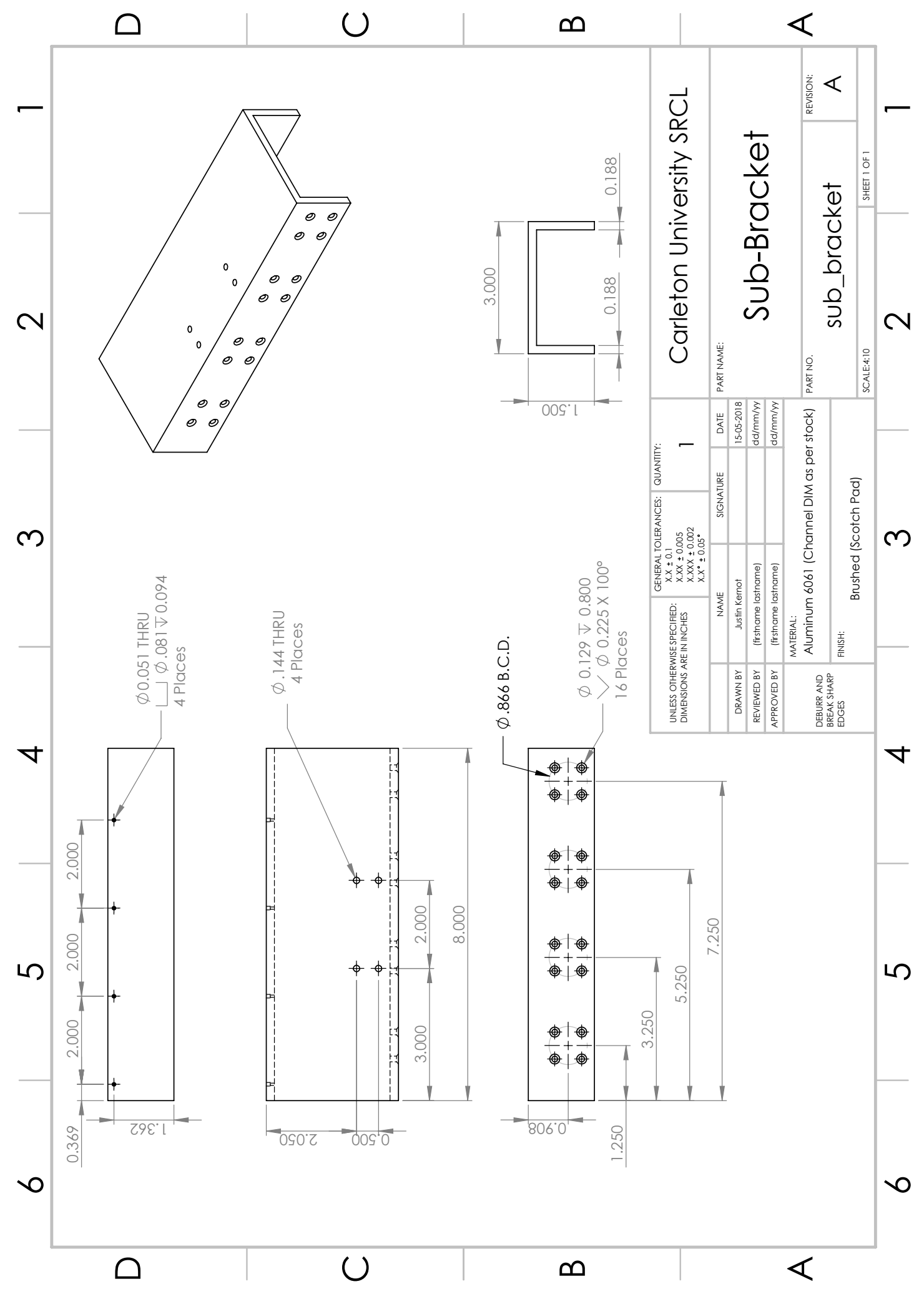




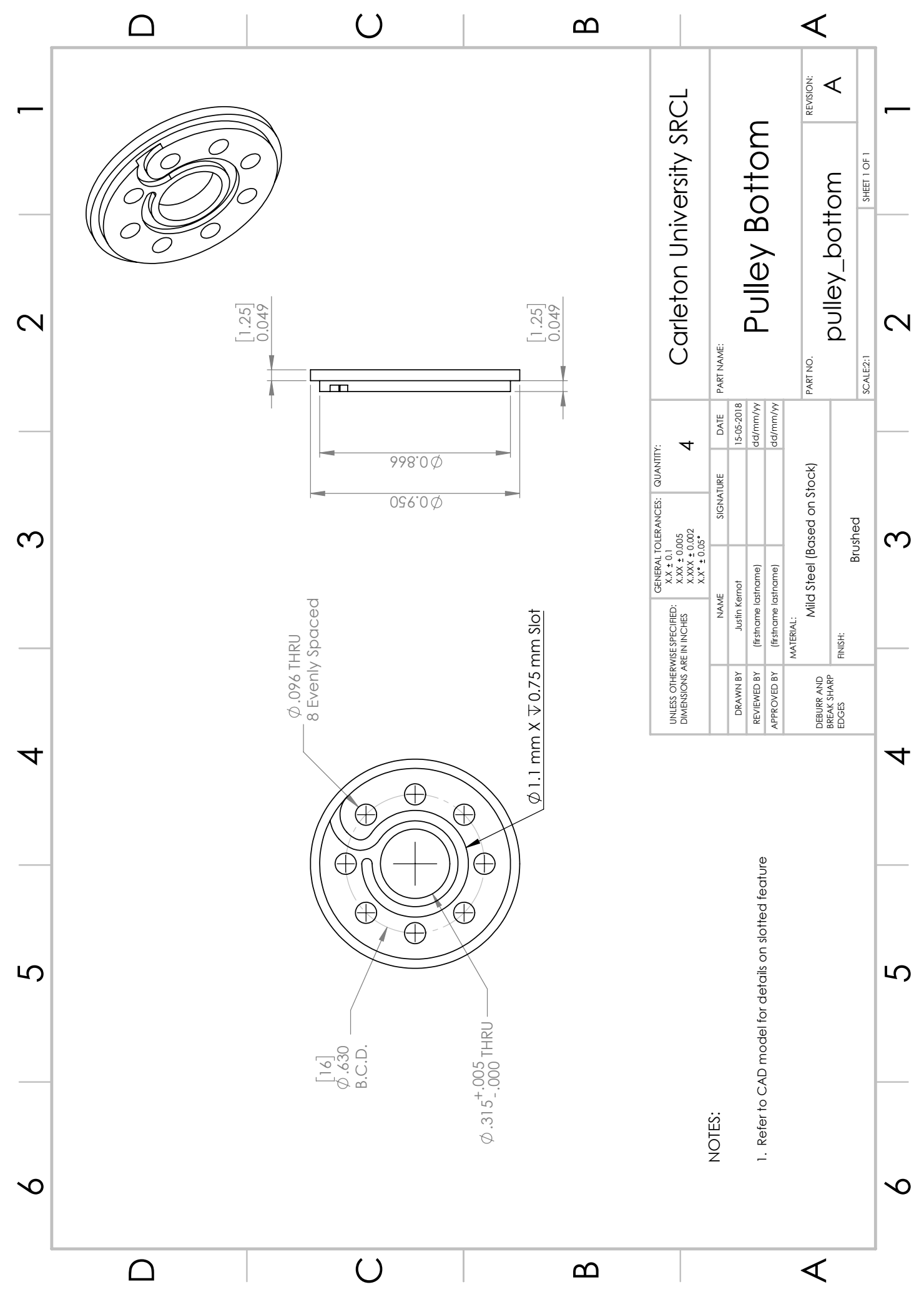




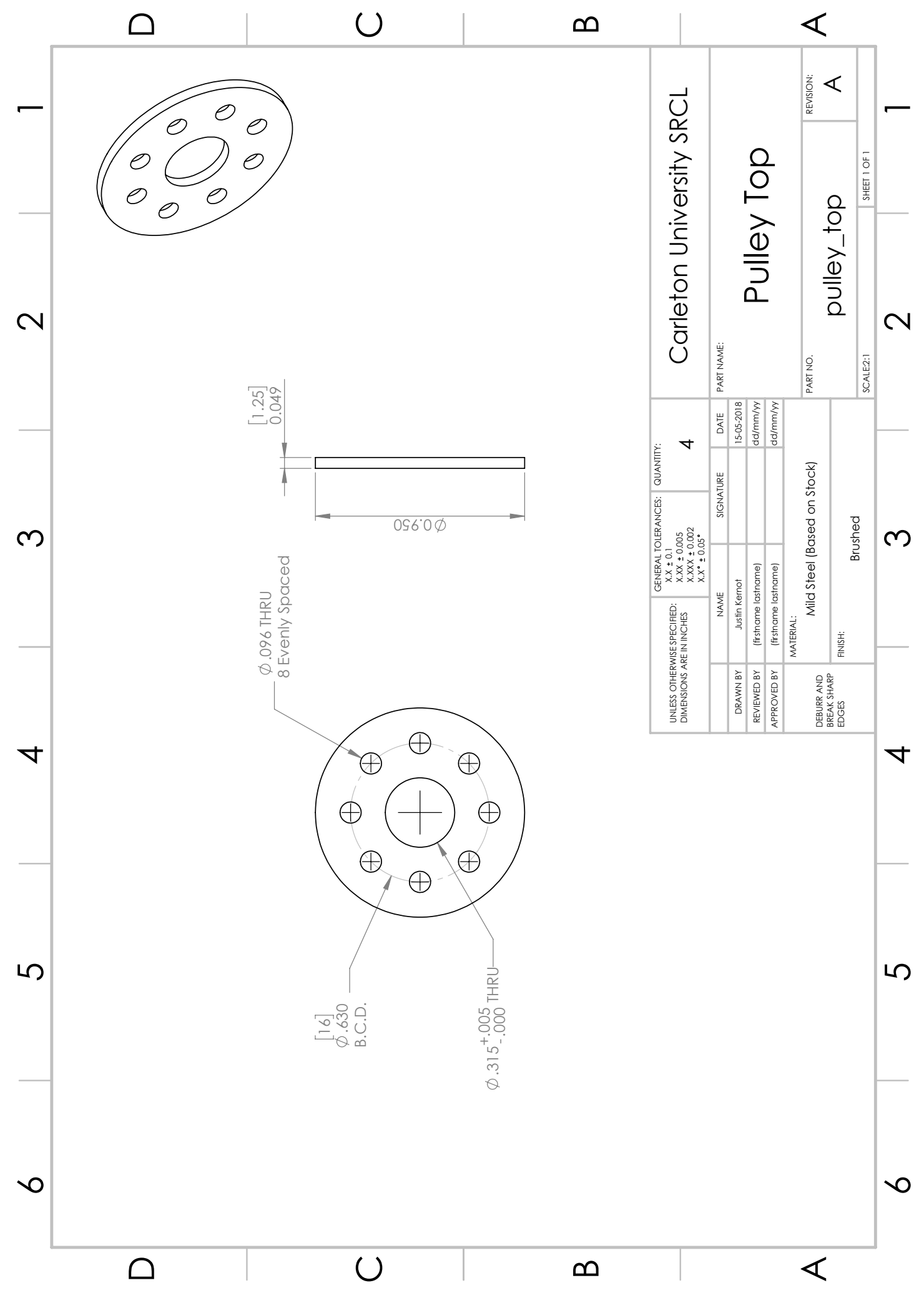




\section{Appendix B}

\section{Code}

This appendix presents the MATLAB scripts and functions used to execute the joint configuration optimization in Section B.1, actuator sizing in Section B.2, the modified Dynamixel 2.0 multi-step script for the identification of the actuation system in Section B.3, the script used to initialize the reference signals (i.e. Sine Wave, Manoevure 1, Manoeuvre 2) in Section B.4, and the controller initialization for the four control cases (i.e. no control, PID, AB, and $\mathcal{L}_{1}$ ) in Section B.5 respectively. 


\section{B.1 Optimization Scripts}

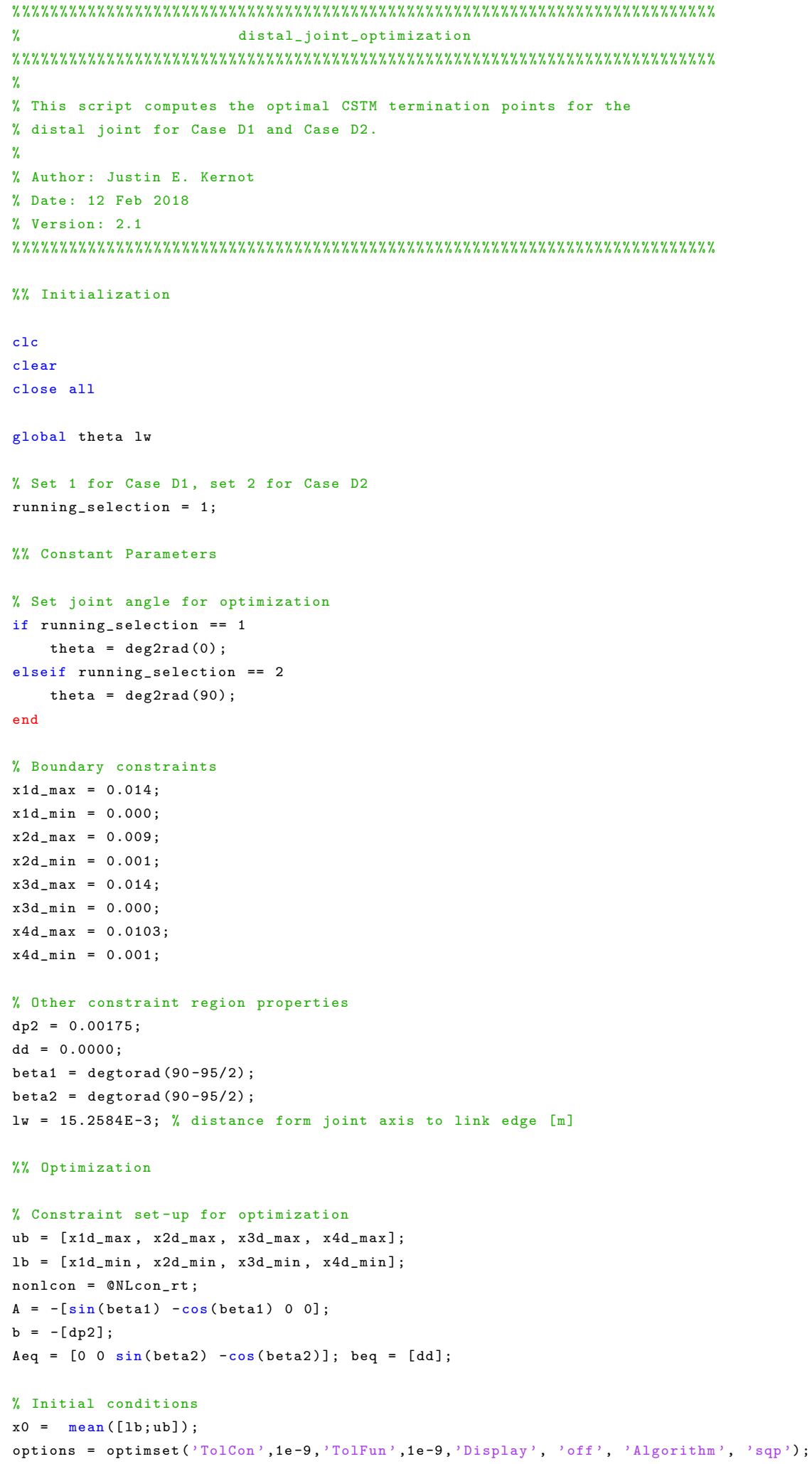




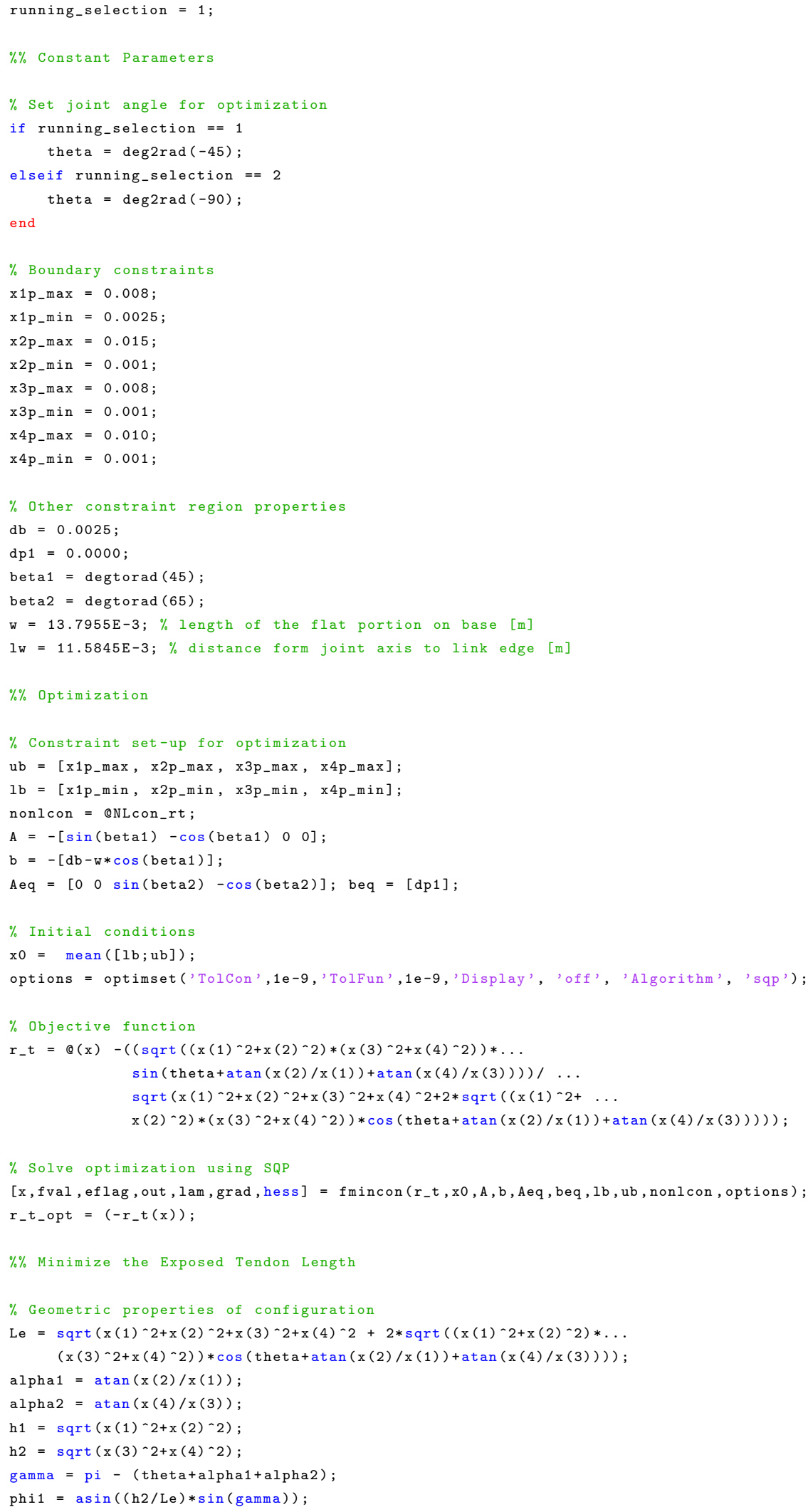




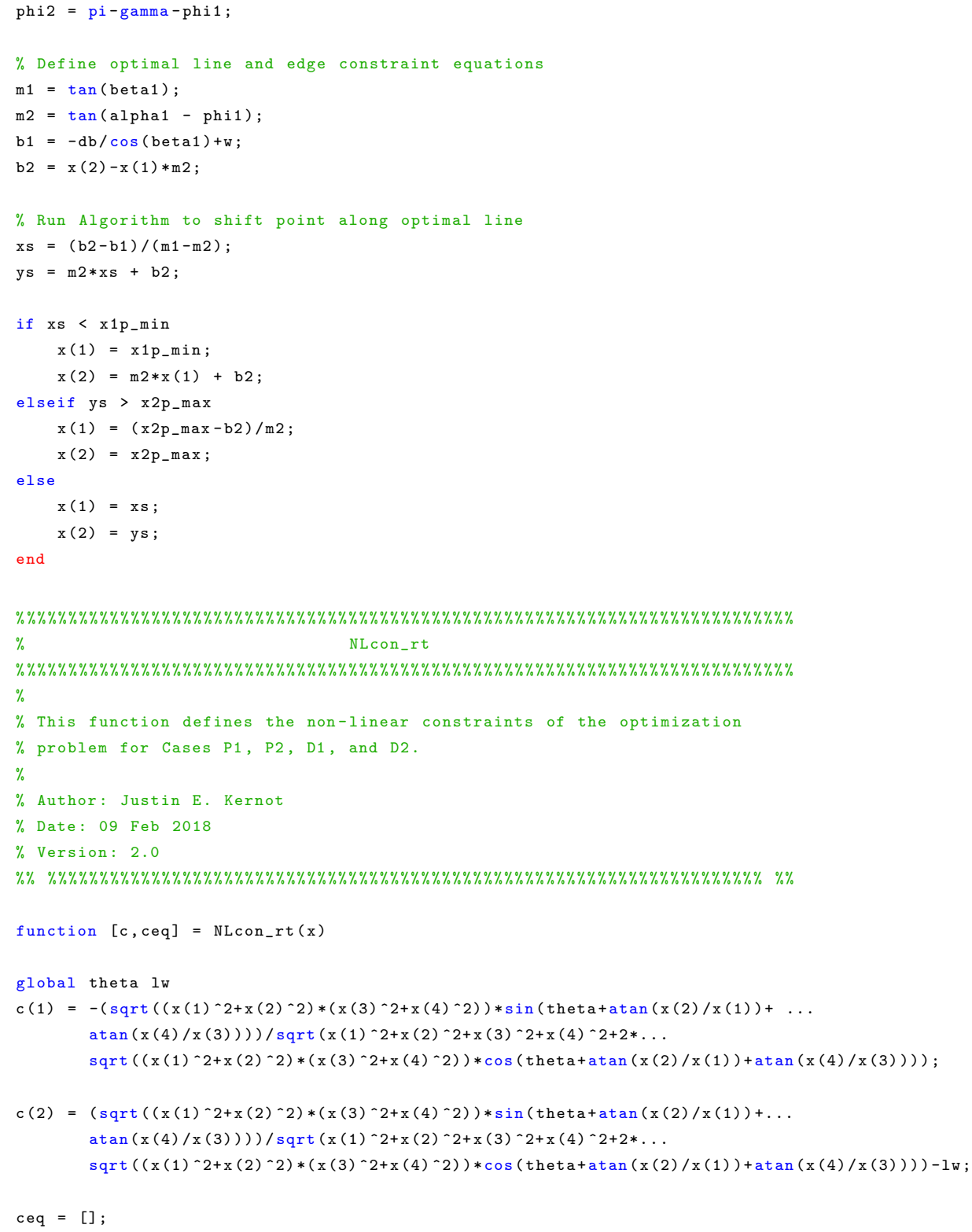




\section{B.2 Actuator Sizing}

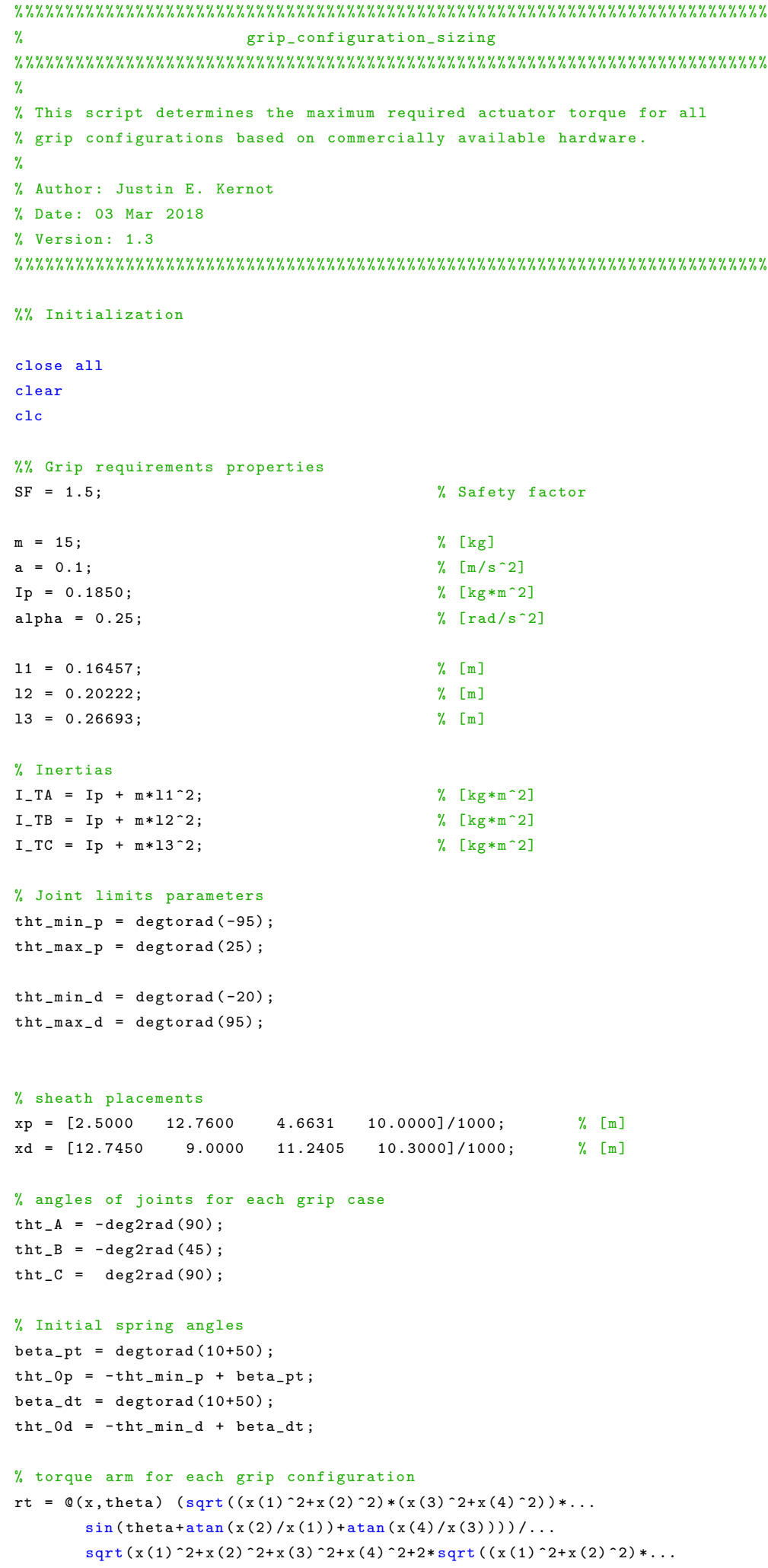




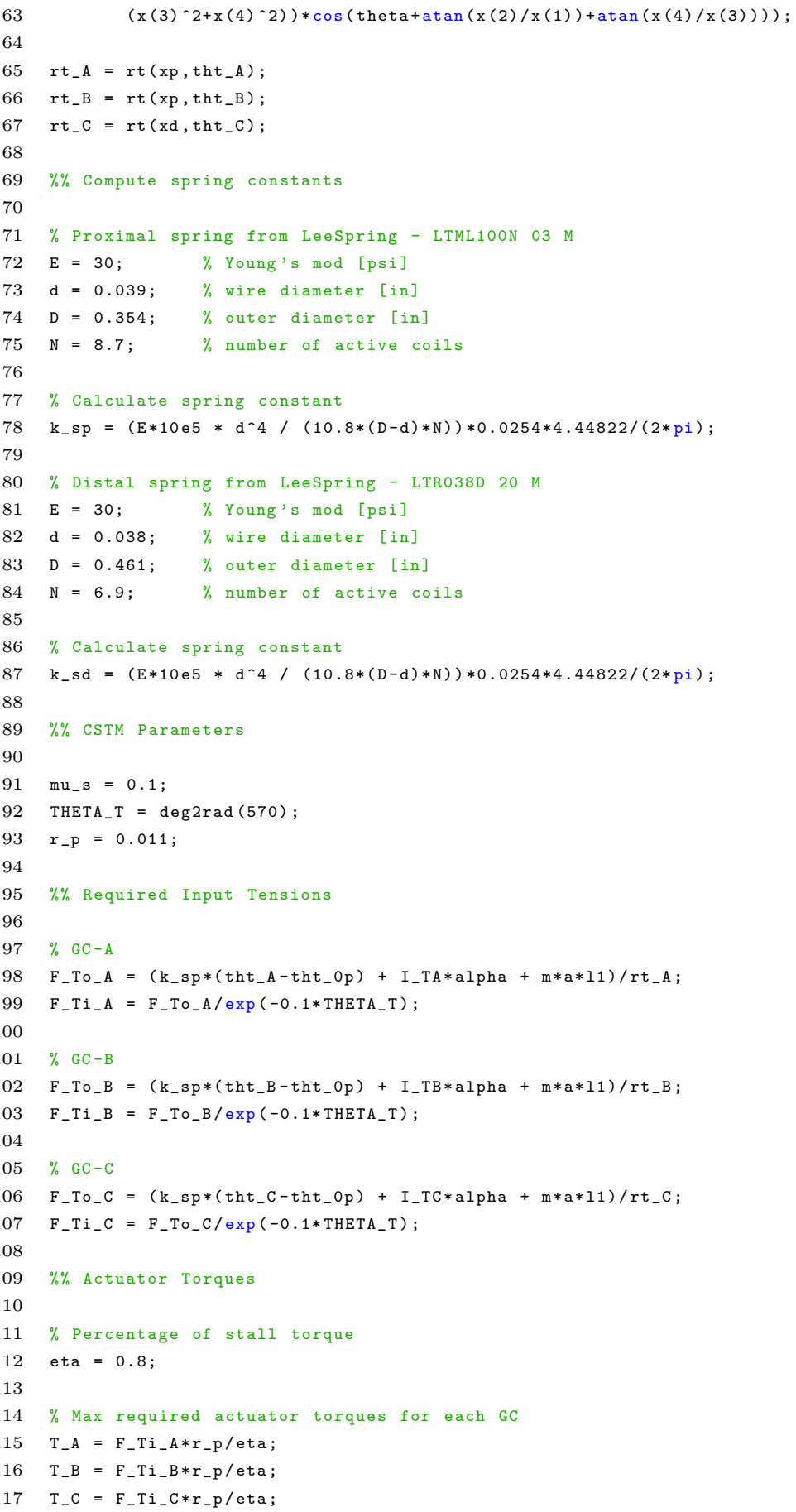




\section{B.3 Multi-step Function for Dynamixel Protocol 2.0}

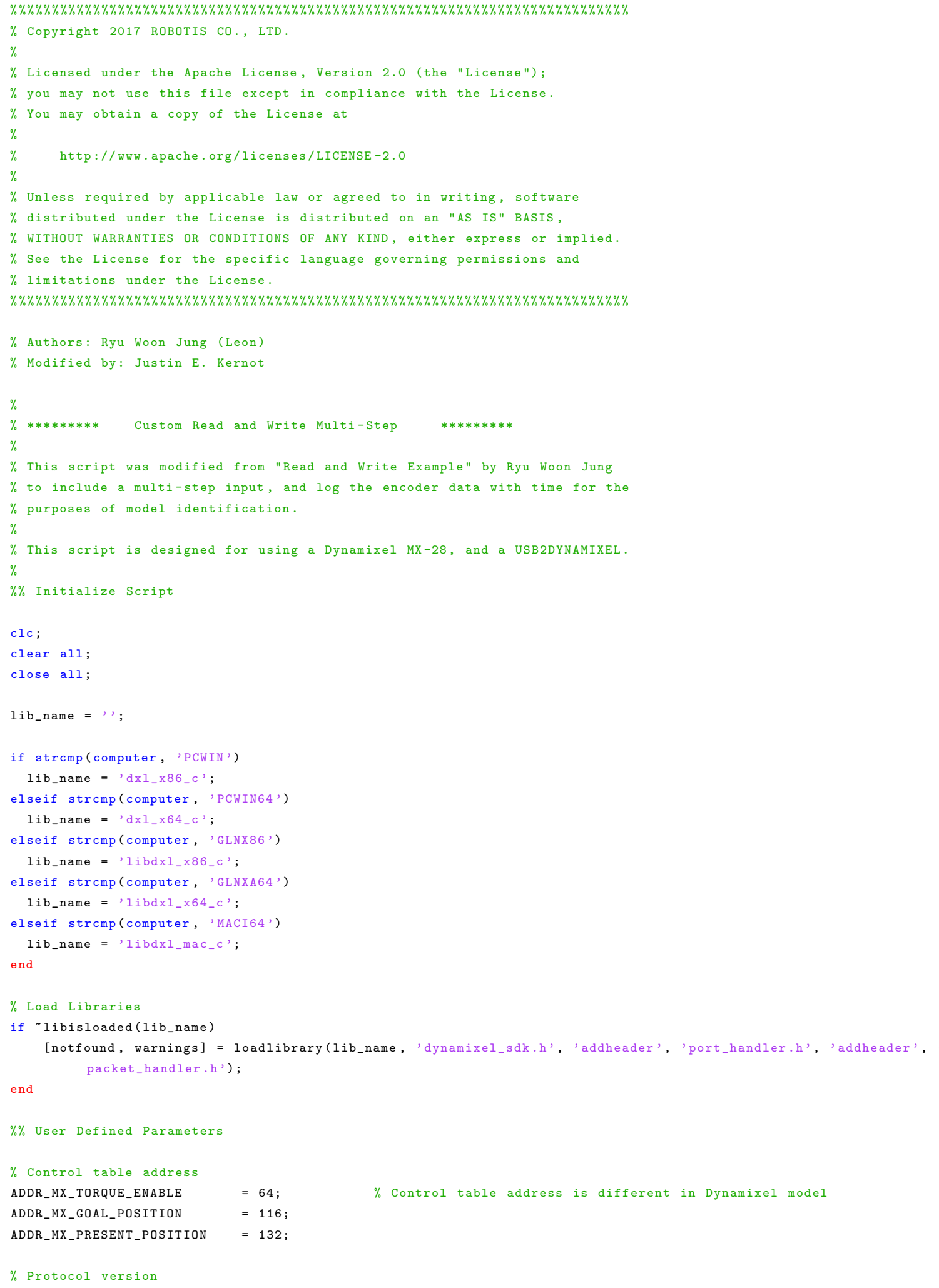




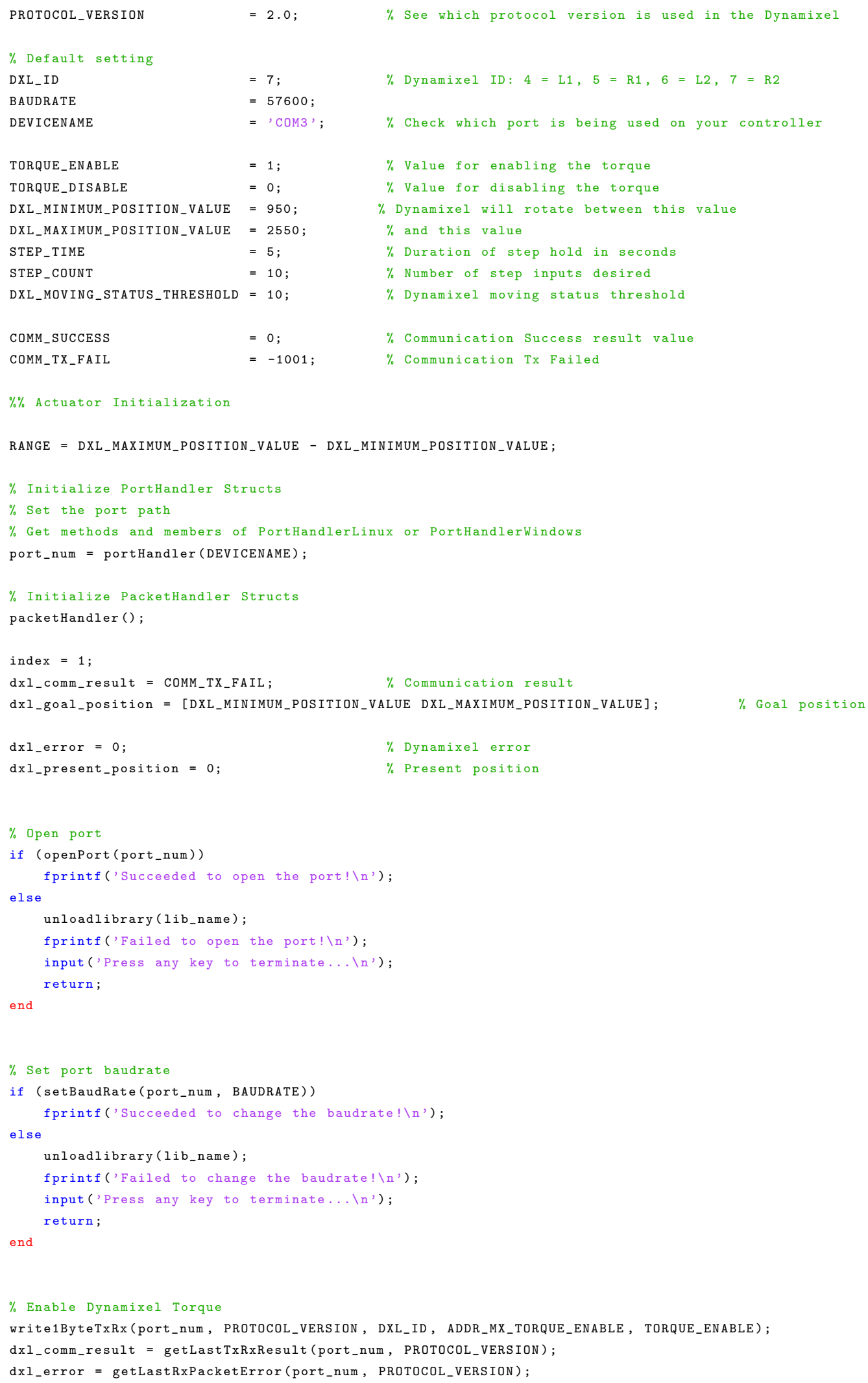




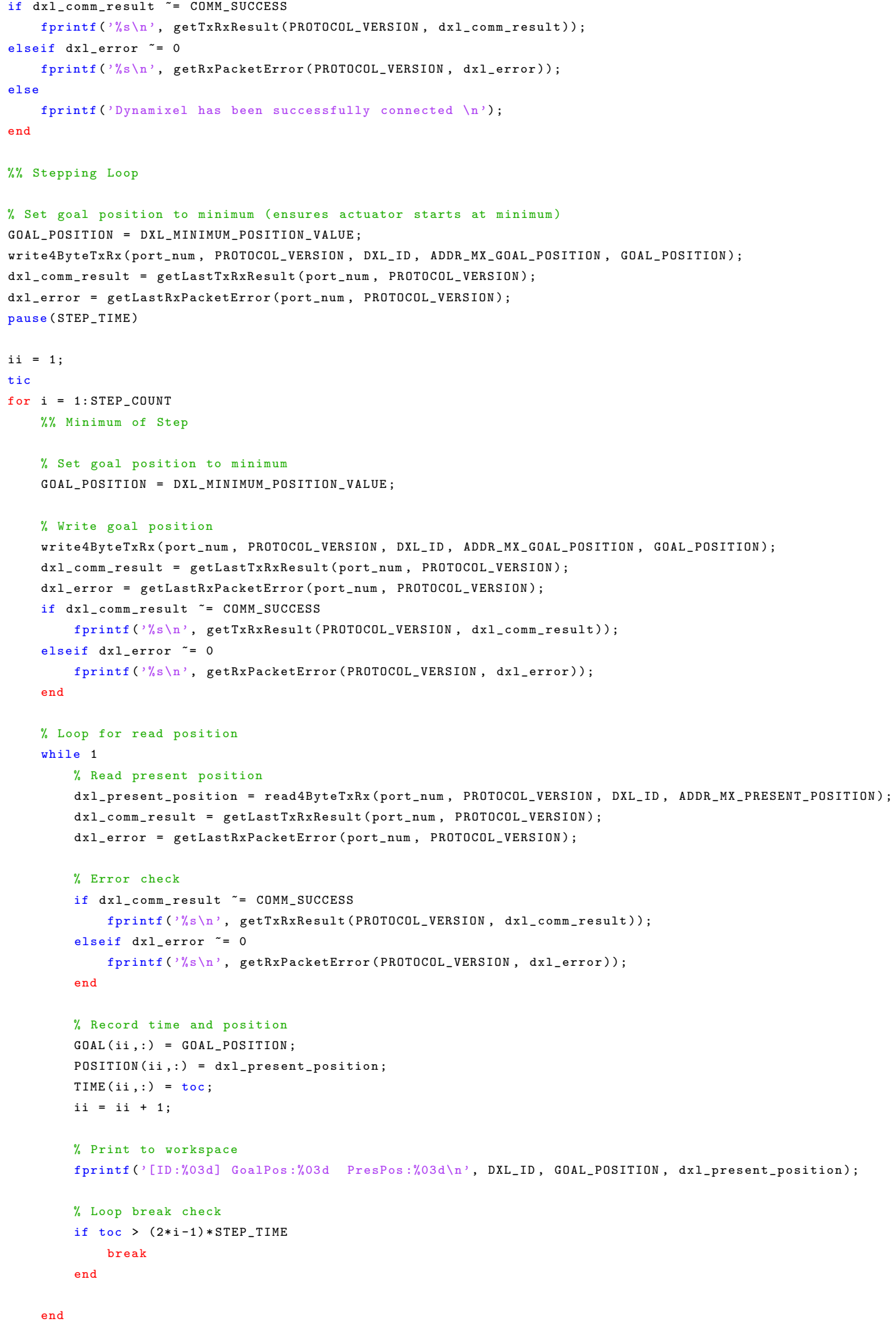




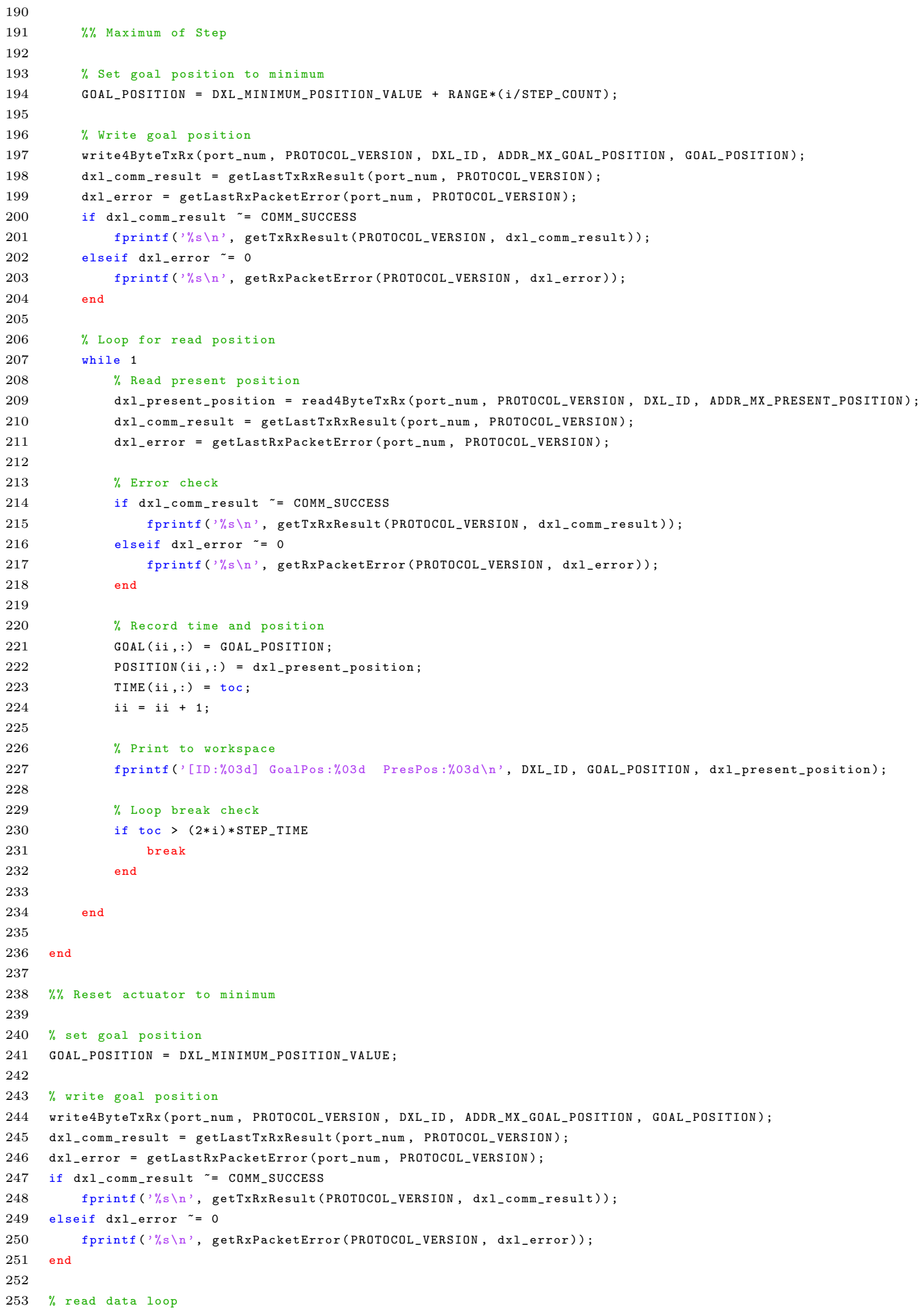




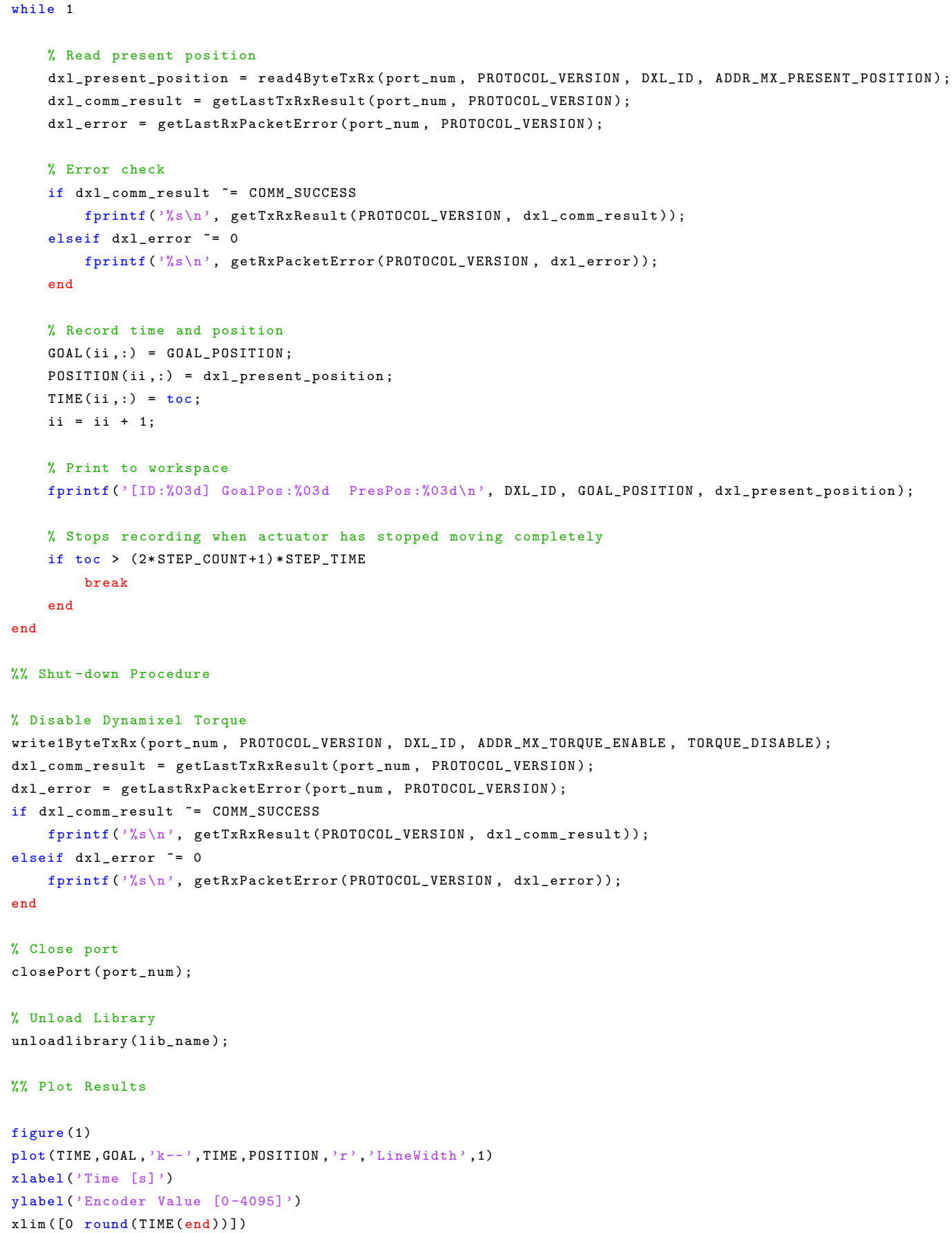




\section{B.4 Manoeuvre Initialization}

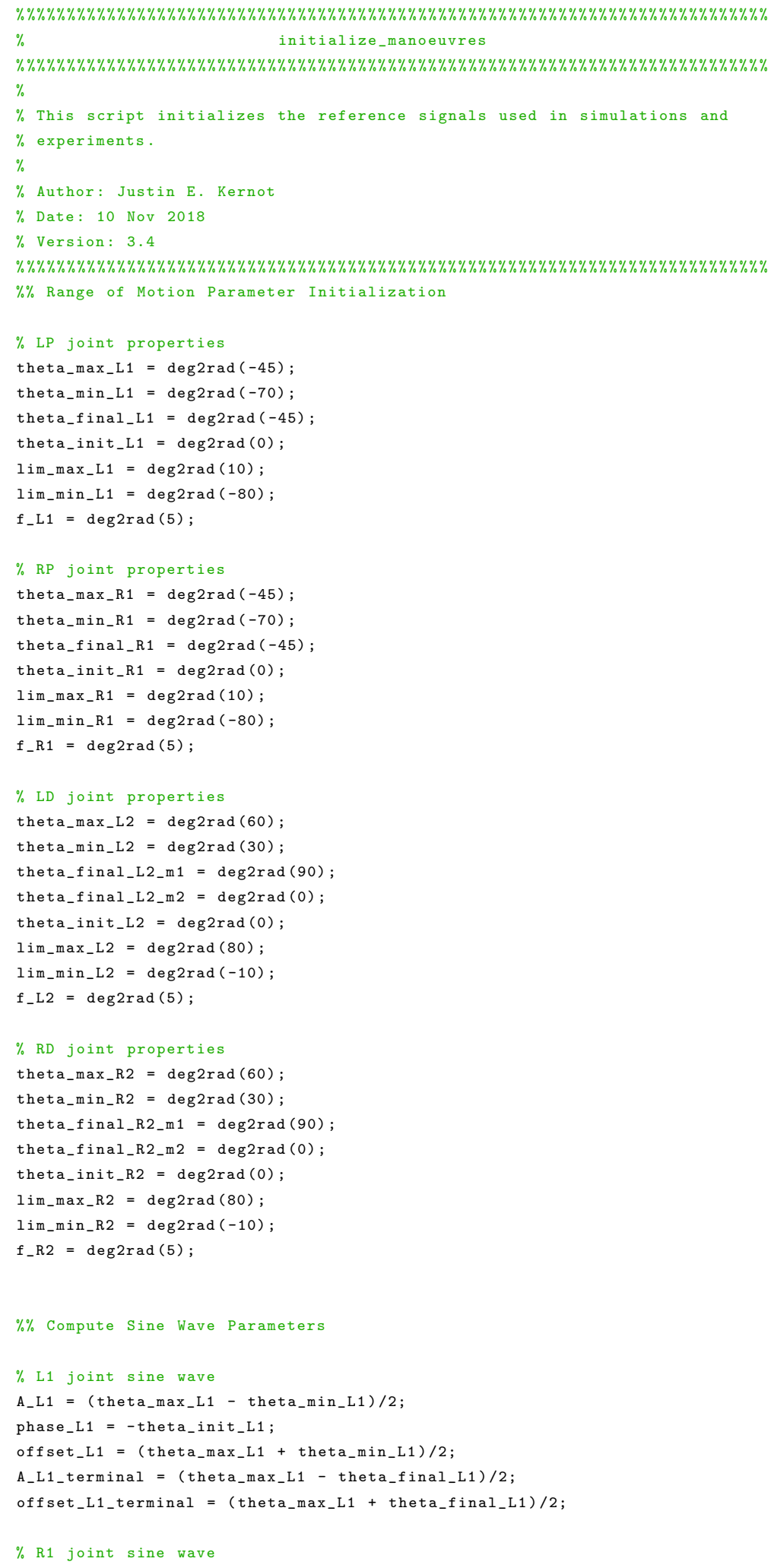




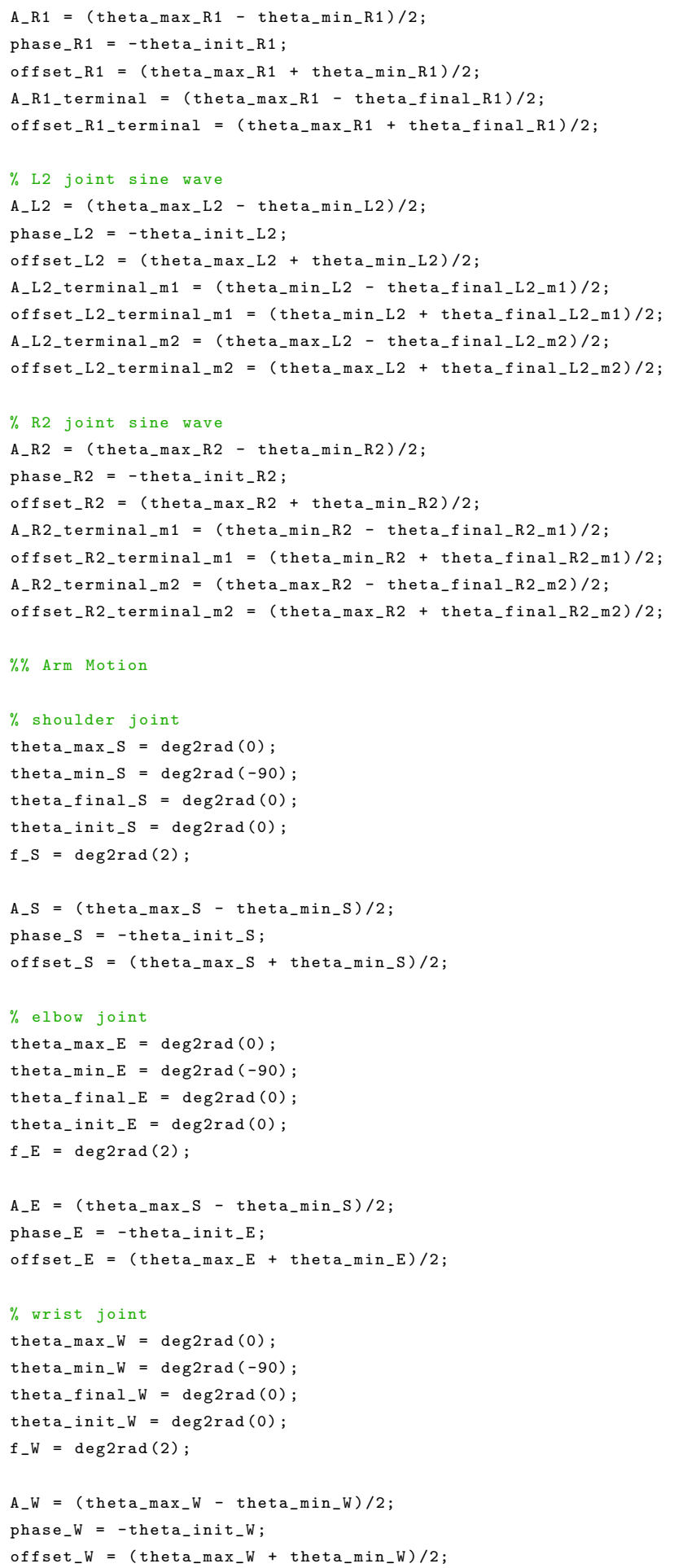




\section{B.5 Controller Initialization}

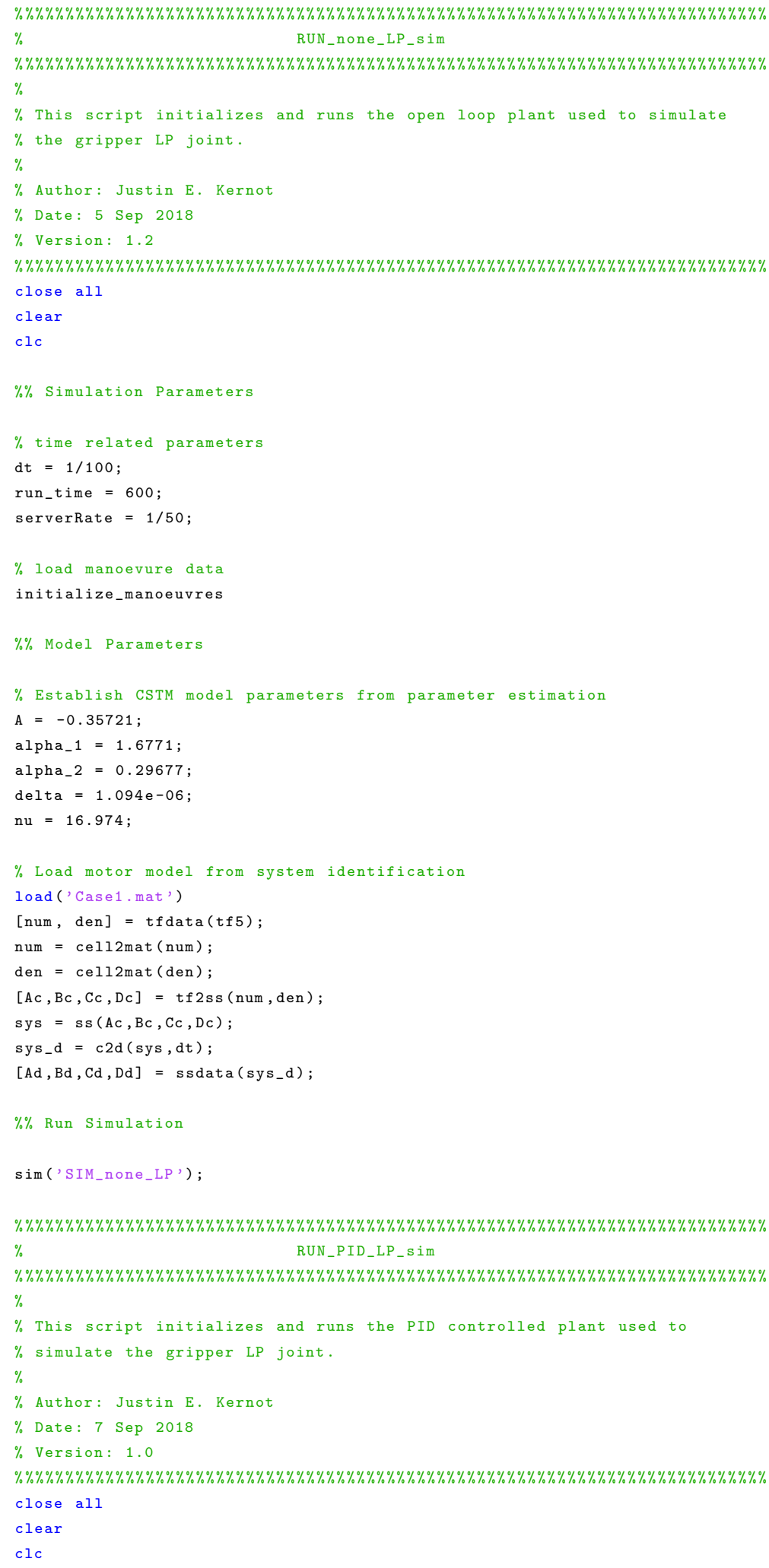




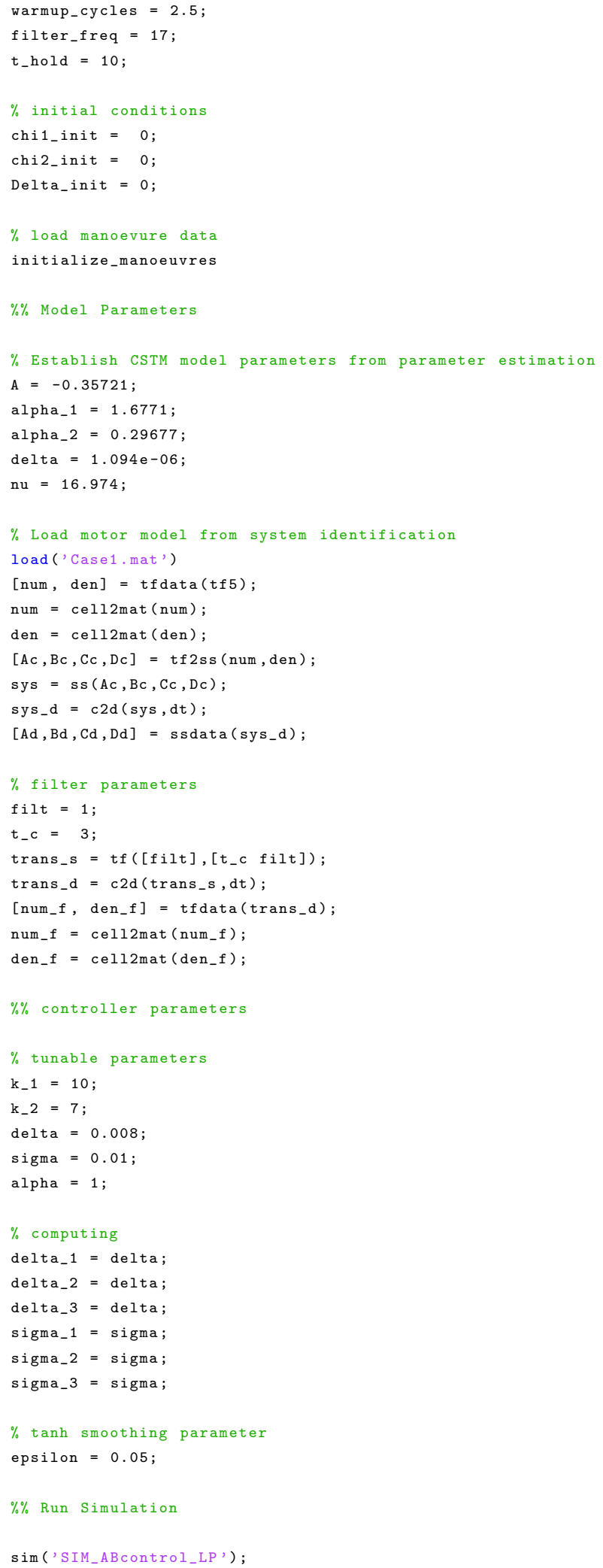




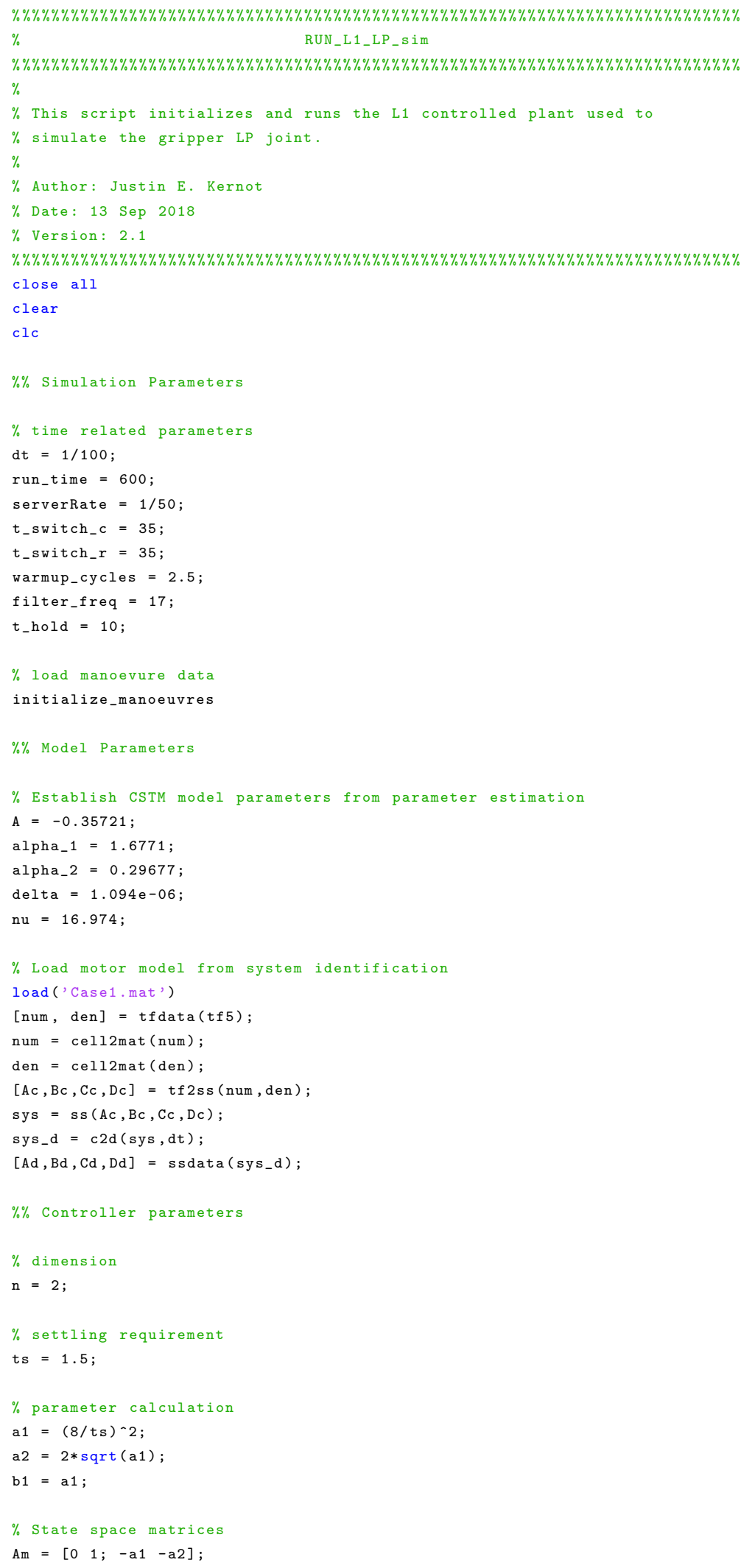




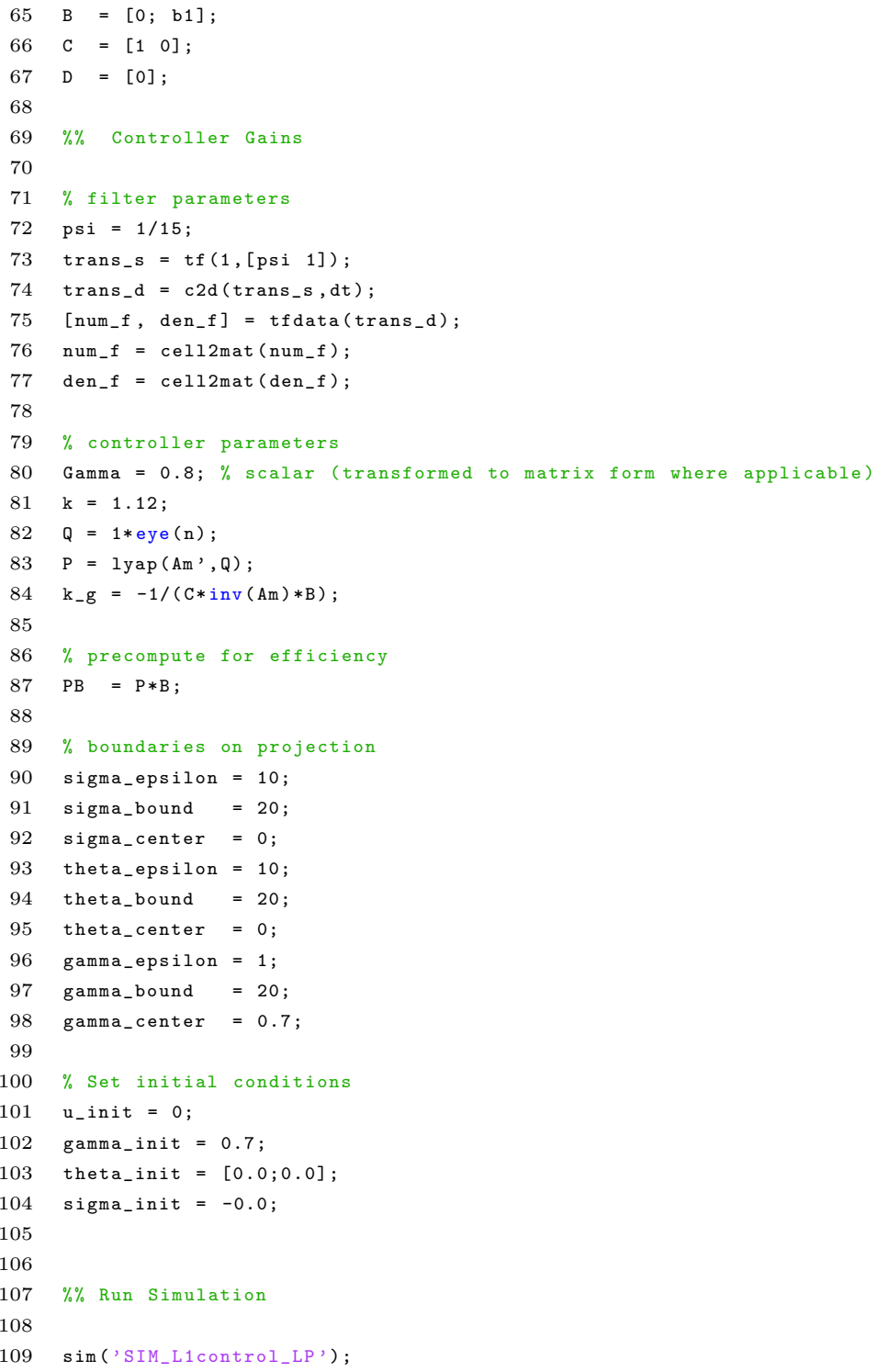




\section{Appendix C}

\section{Supplementary Data}

This appendix presents the supplementary data for the experimental cases that were not presented in Chapter 6 .

\section{C.1 No Control}

\section{C.1.1 LP Joint Data}
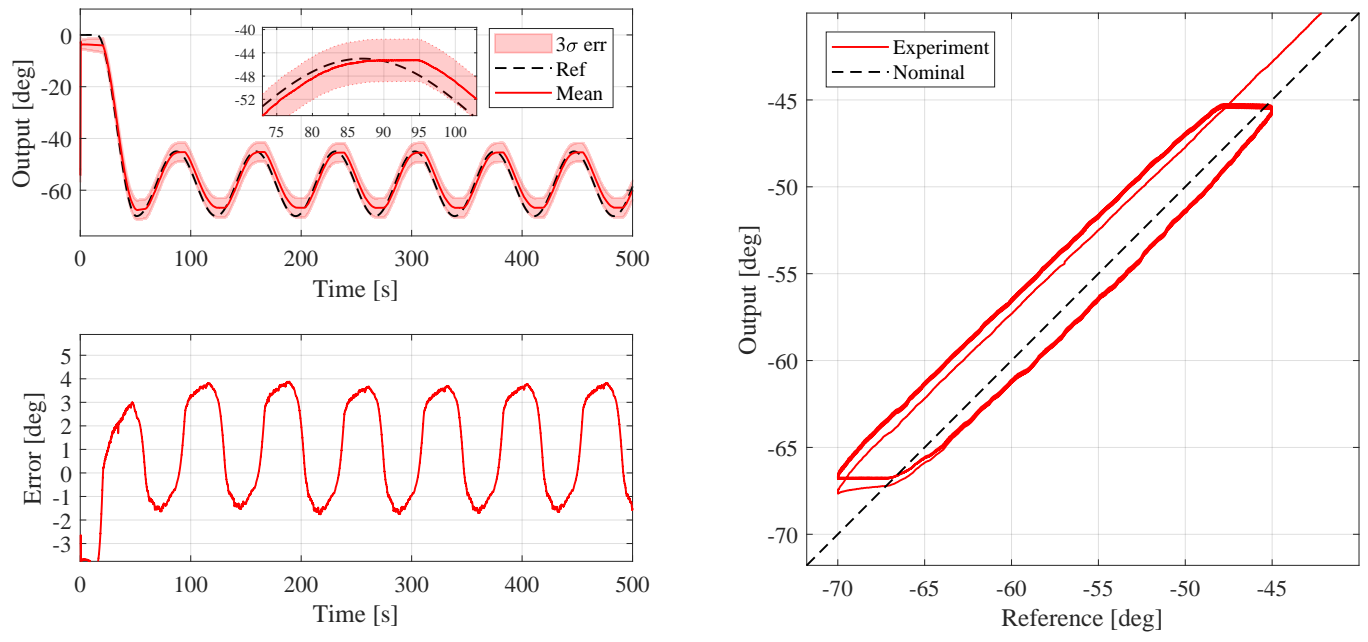

Figure C.1: LP joint performance for the Sine Wave manoeuvre in a straight arm configuration under no control. Top: actual and desired proximal link angular trajectory, bottom: mean trajectory tracking error, and right: mean hysteresis loop of transient phase. 

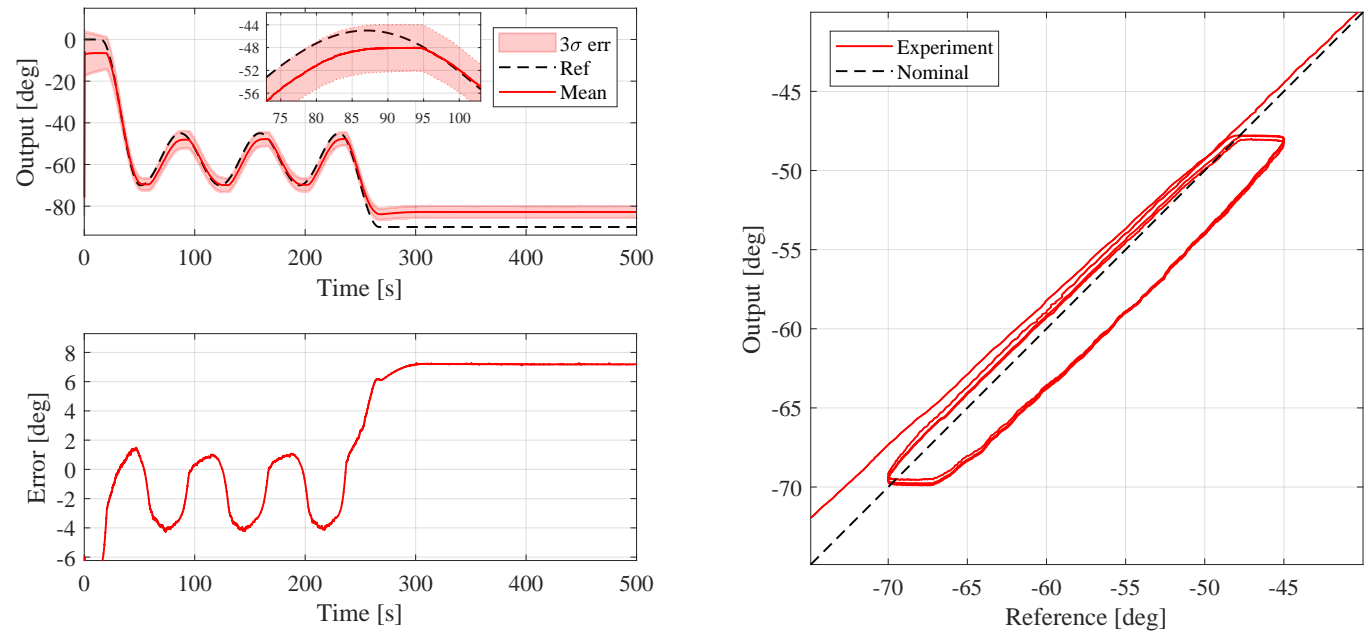

Figure C.2: LP joint performance for Manoeuvre 2 in a straight arm configuration under no control. Top: actual and desired proximal link angular trajectory, bottom: mean trajectory tracking error, and right: mean hysteresis loop of transient phase.
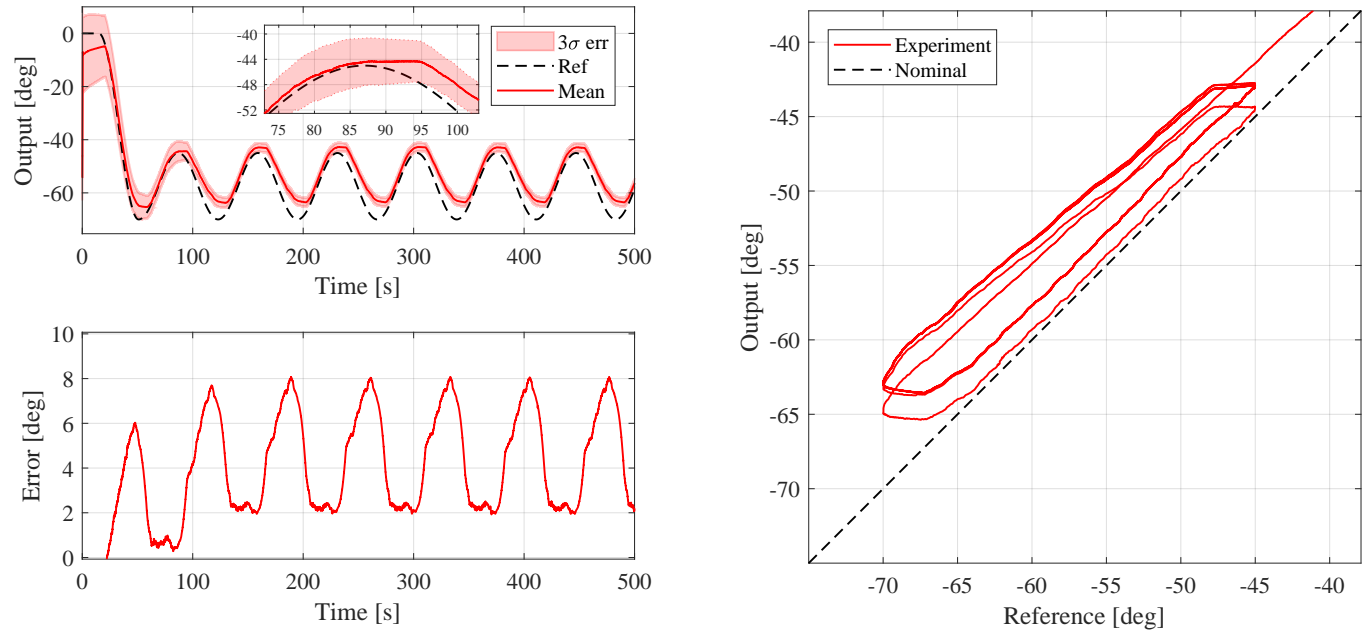

Figure C.3: LP joint performance for the Sine Wave manoeuvre in a bent arm configuration under no control. Top: actual and desired proximal link angular trajectory, bottom: mean trajectory tracking error, and right: mean hysteresis loop of transient phase. 

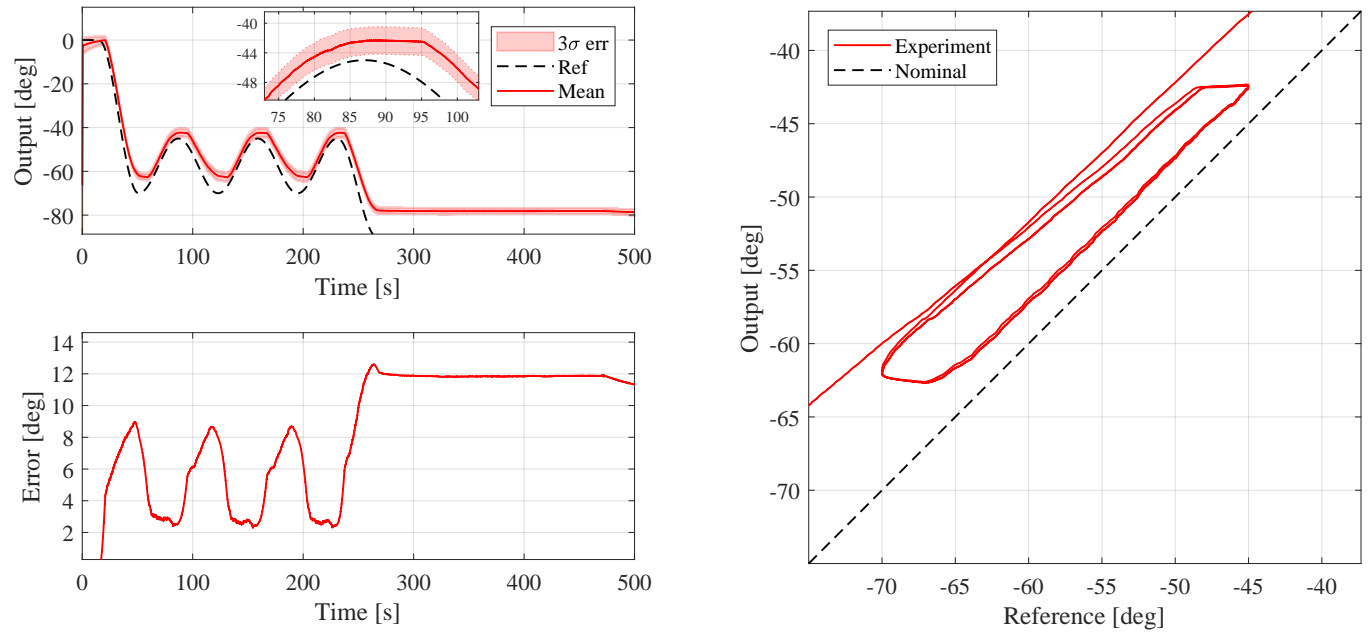

Figure C.4: LP joint performance for Manoeuvre 2 in a bent arm configuration under no control. Top: actual and desired proximal link angular trajectory, bottom: mean trajectory tracking error, and right: mean hysteresis loop of transient phase.

\section{C.1.2 LD Joint Data}
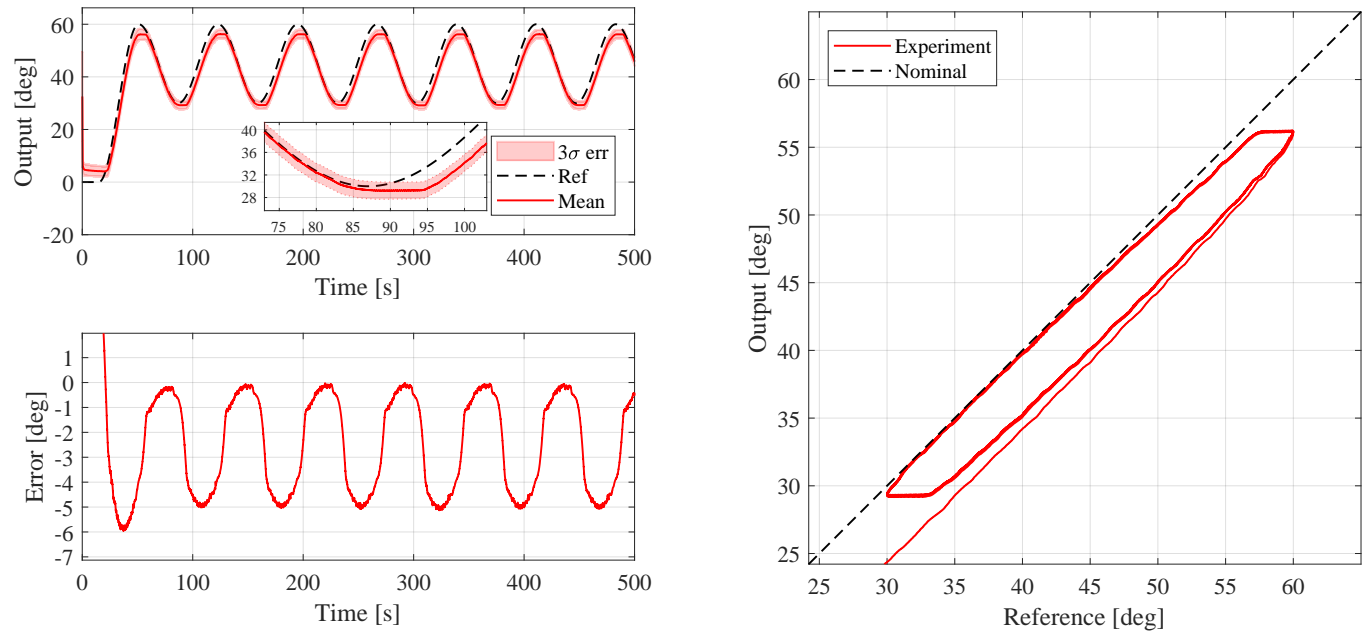

Figure C.5: LD joint performance for the Sine Wave manoeuvre in a straight arm configuration under no control. Top: actual and desired proximal link angular trajectory, bottom: mean trajectory tracking error, and right: mean hysteresis loop of transient phase. 

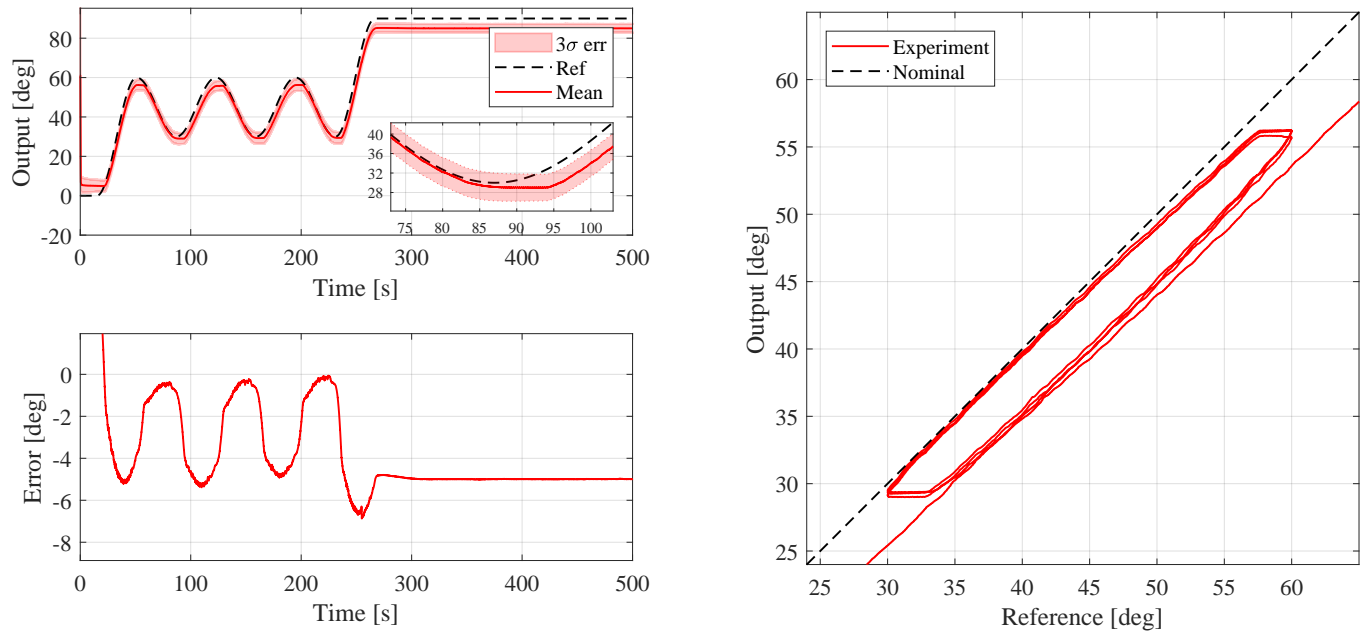

Figure C.6: LD joint performance for Manoeuvre 1 in a straight arm configuration under no control. Top: actual and desired proximal link angular trajectory, bottom: mean trajectory tracking error, and right: mean hysteresis loop of transient phase.
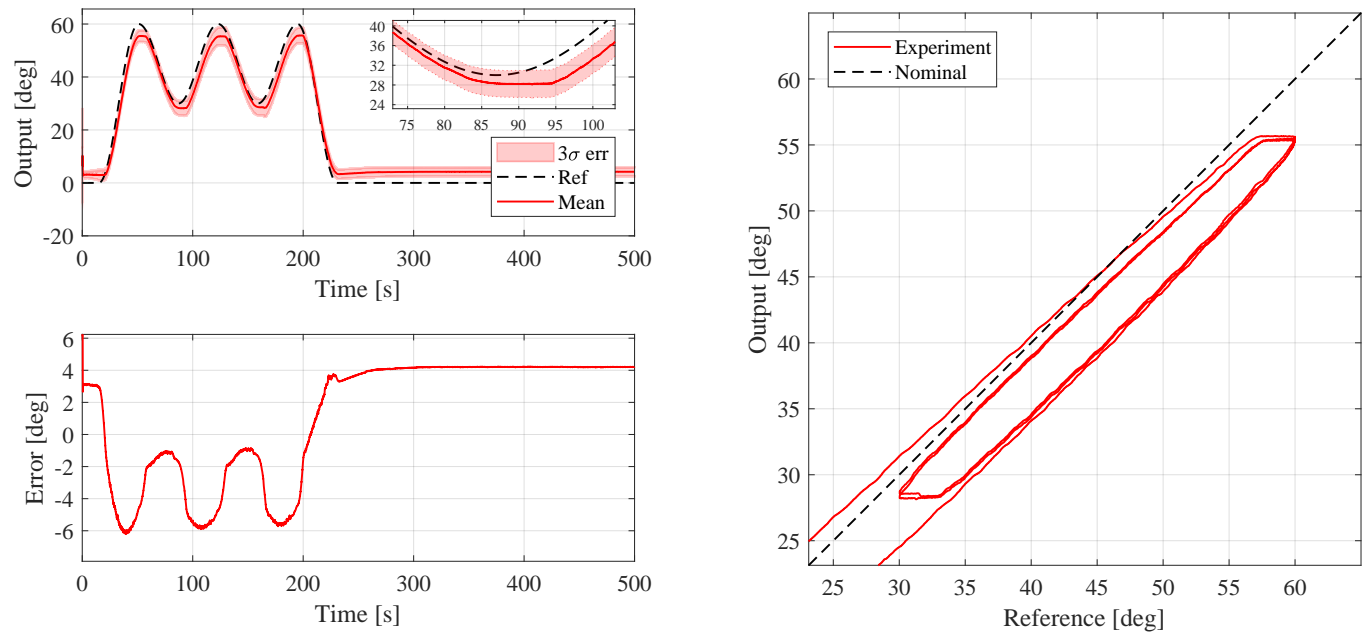

Figure C.7: LD joint performance for Manoeuvre 2 in a straight arm configuration under no control. Top: actual and desired proximal link angular trajectory, bottom: mean trajectory tracking error, and right: mean hysteresis loop of transient phase. 

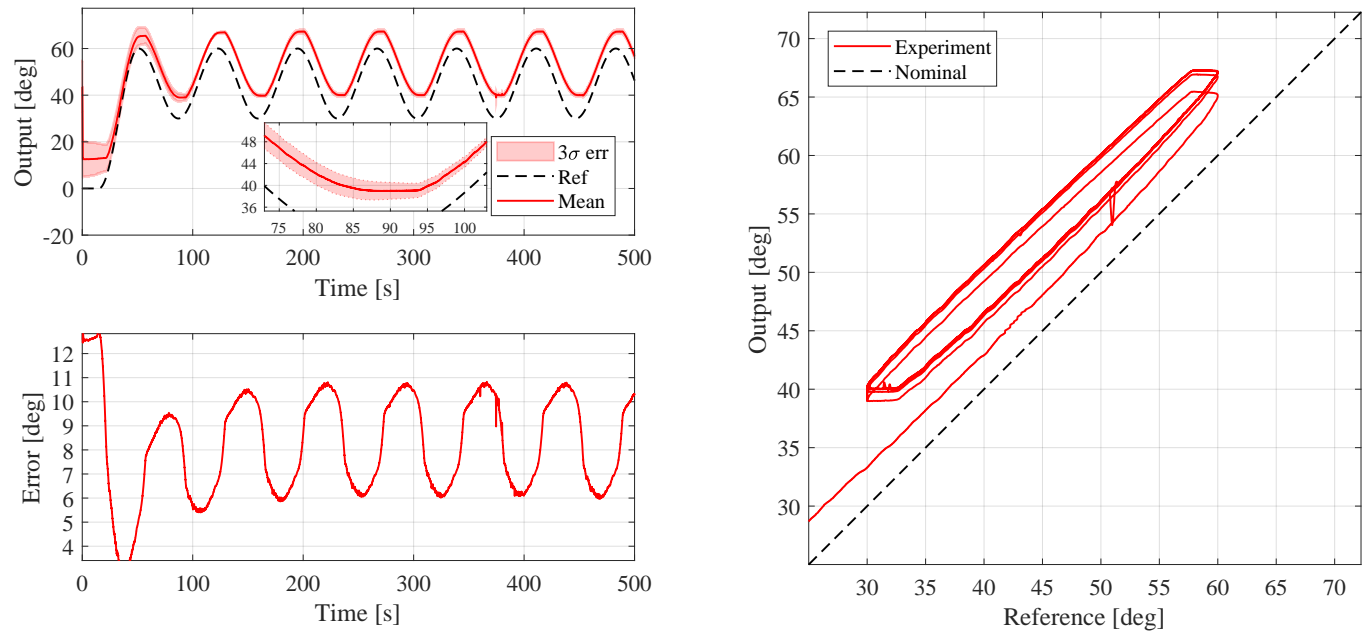

Figure C.8: LD joint performance for the Sine Wave manoeuvre in a bent arm configuration under no control. Top: actual and desired proximal link angular trajectory, bottom: mean trajectory tracking error, and right: mean hysteresis loop of transient phase.
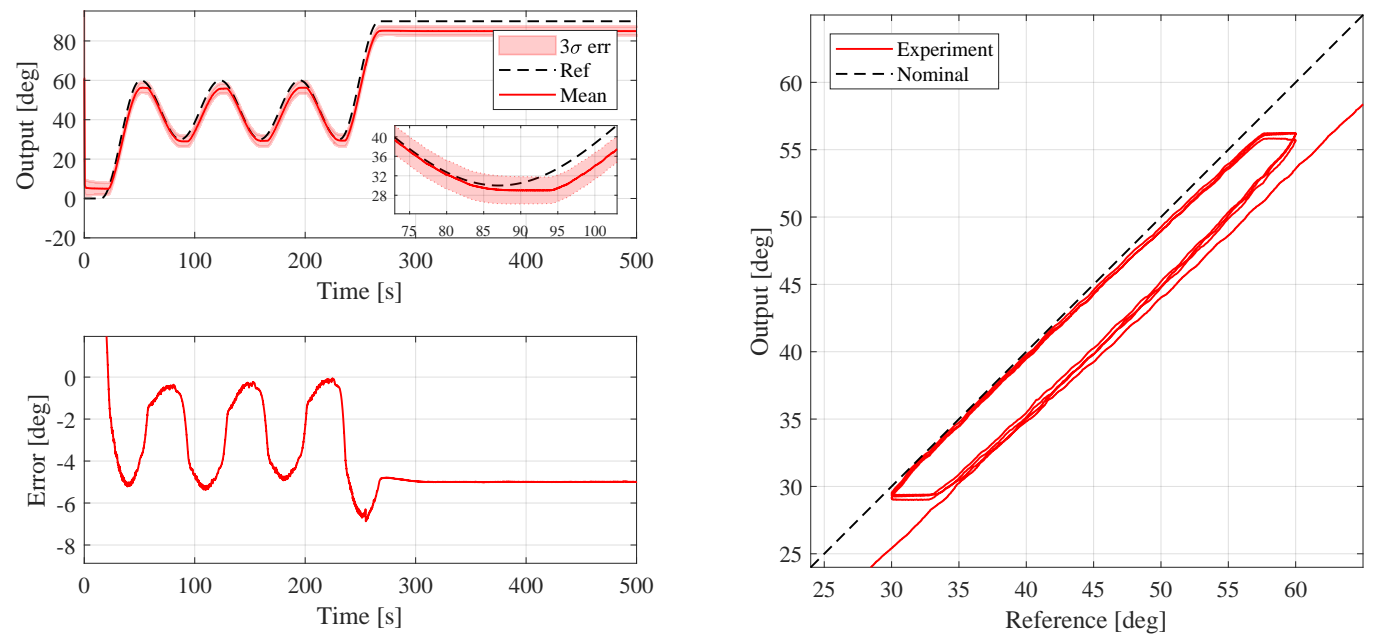

Figure C.9: LD joint performance for Manoeuvre 1 in a straight arm configuration under no control. Top: actual and desired proximal link angular trajectory, bottom: mean trajectory tracking error, and right: mean hysteresis loop of transient phase. 

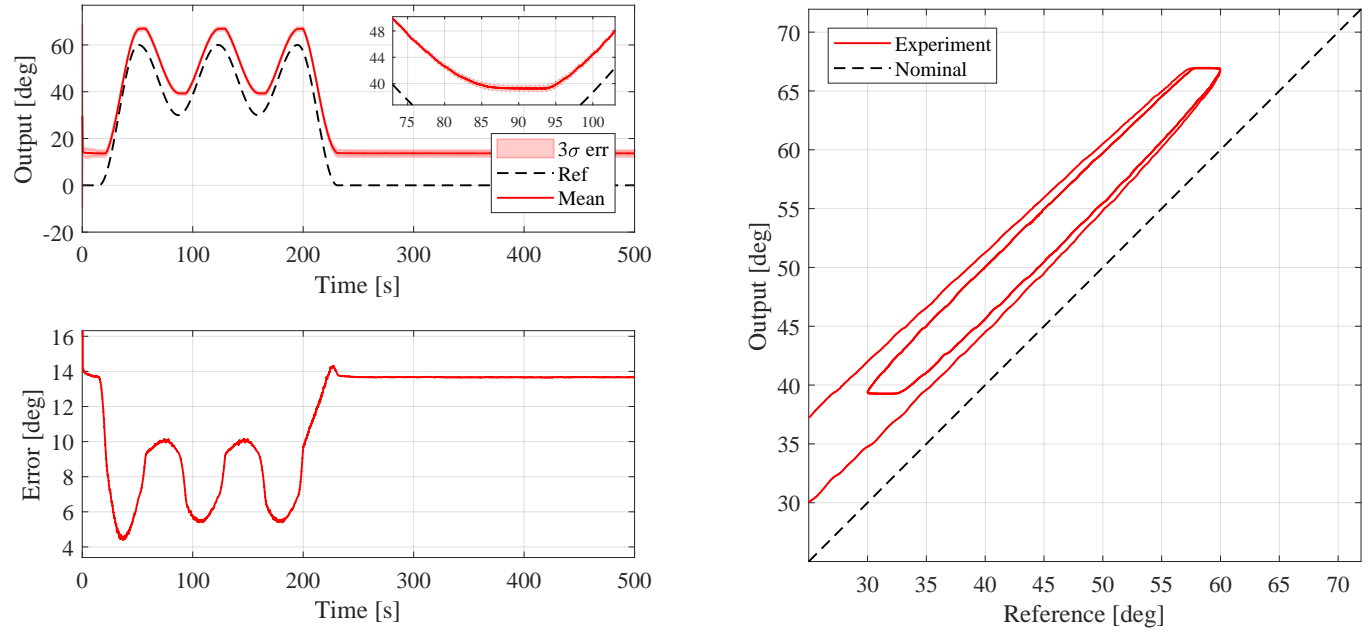

Figure C.10: LD joint performance for Manoeuvre 2 in a bent arm configuration under no control. Top: actual and desired proximal link angular trajectory, bottom: mean trajectory tracking error, and right: mean hysteresis loop of transient phase.
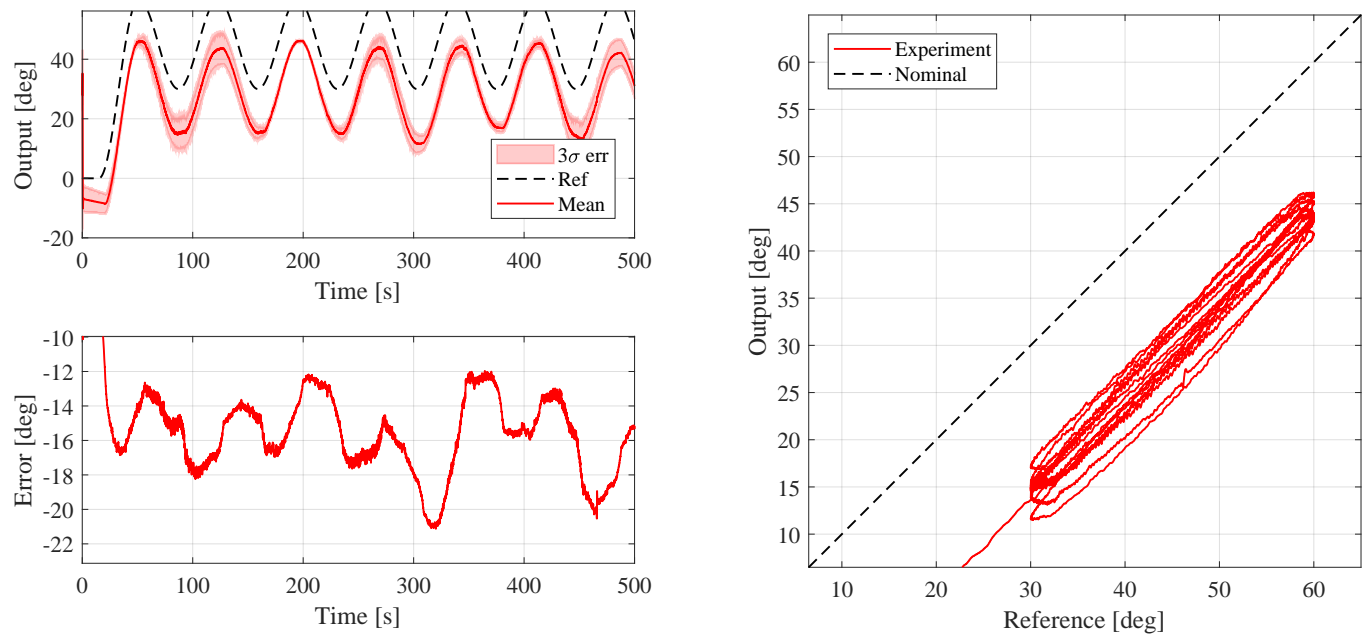

Figure C.11: LD joint performance with moving arm under no control. Top: actual and desired proximal link angular trajectory, bottom: mean trajectory tracking error, and right: mean hysteresis loop of transient phase. 


\section{C.2 PID Control}

\section{C.2.1 LP Joint Data}
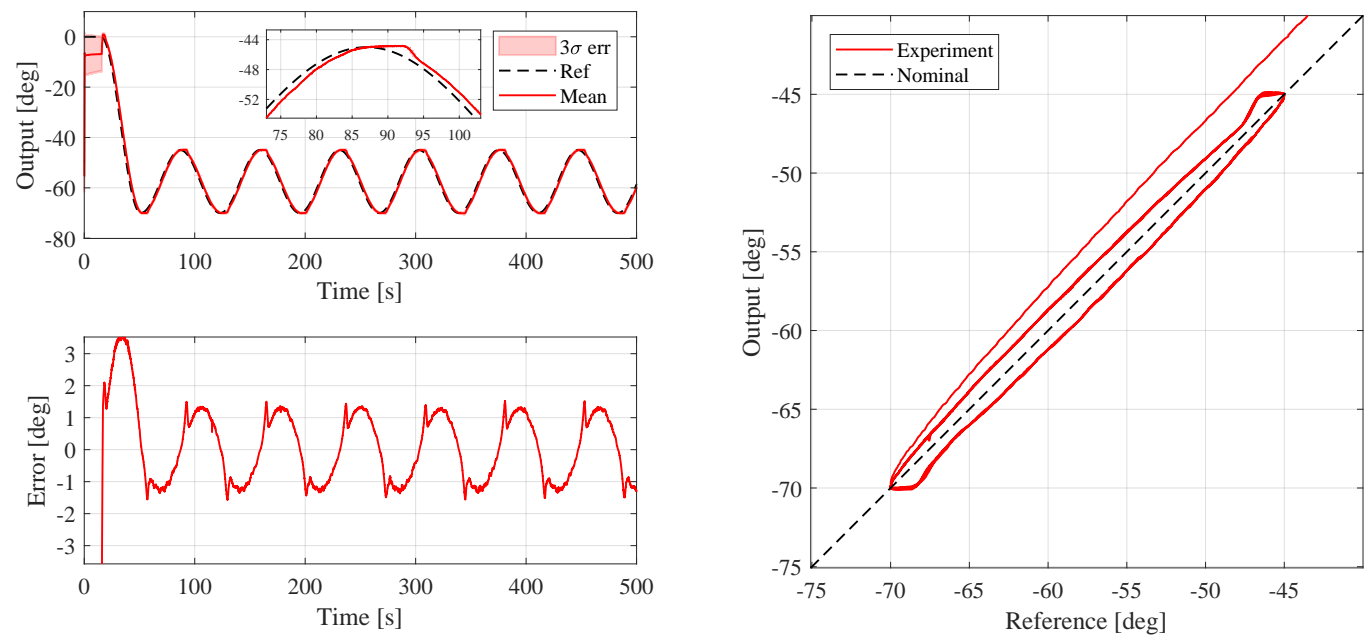

Figure C.12: LP joint performance for the Sine Wave manoeuvre in a straight arm configuration under PID control. Top: actual and desired proximal link angular trajectory, bottom: mean trajectory tracking error, and right: mean hysteresis loop of transient phase.
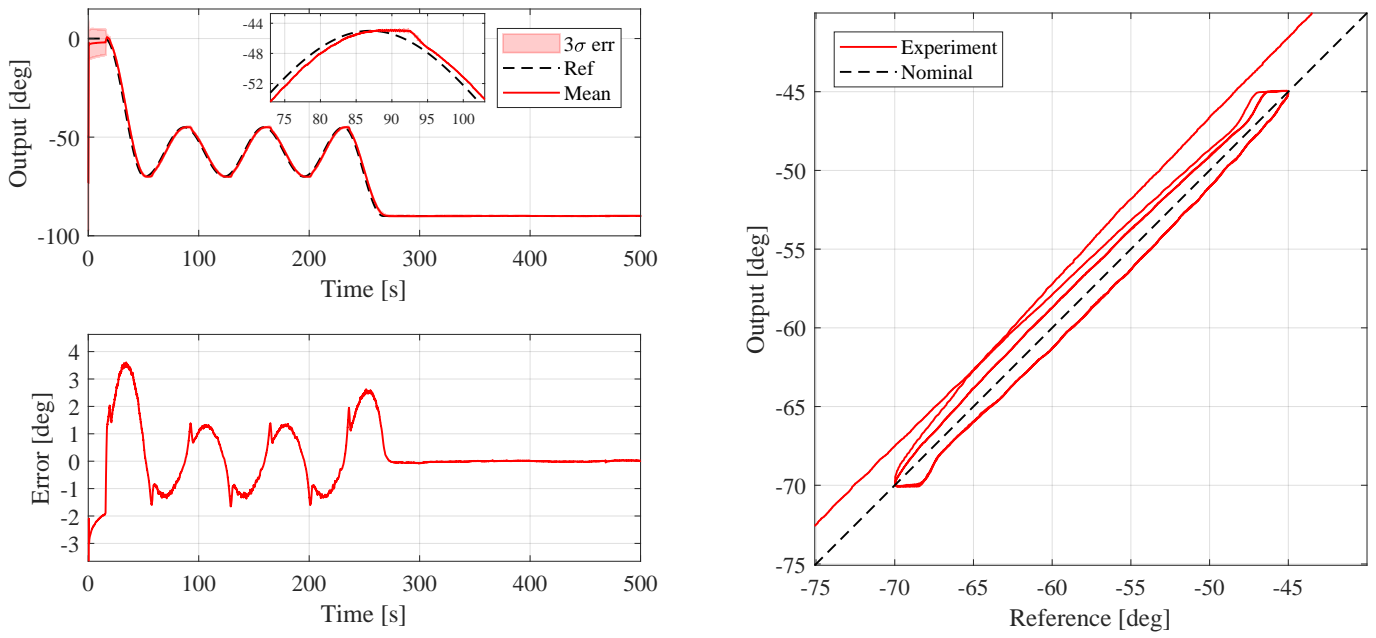

Figure C.13: LP joint performance for Manoeuvre 2 in a straight arm configuration under PID control. Top: actual and desired proximal link angular trajectory, bottom: mean trajectory tracking error, and right: mean hysteresis loop of transient phase. 

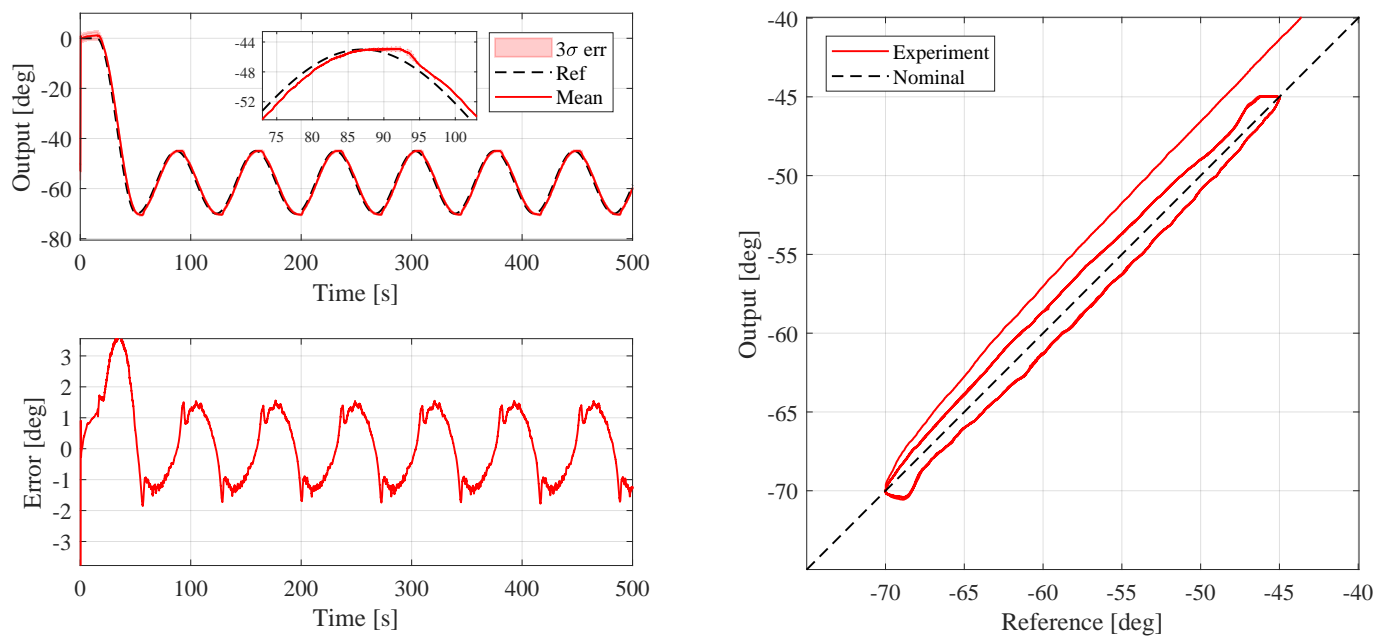

Figure C.14: LP joint performance for the Sine Wave manoeuvre in a bent arm configuration under PID control. Top: actual and desired proximal link angular trajectory, bottom: mean trajectory tracking error, and right: mean hysteresis loop of transient phase.
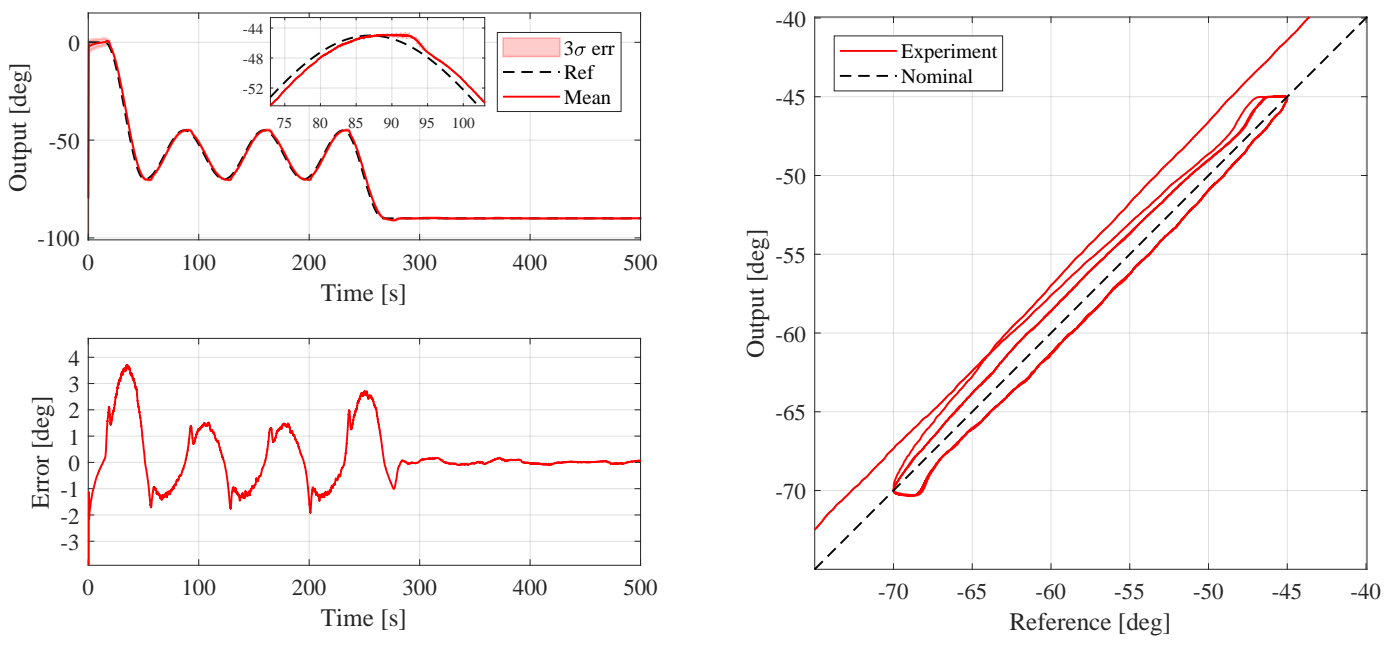

Figure C.15: LP joint performance for Manoeuvre 2 in a bent arm configuration under PID control. Top: actual and desired proximal link angular trajectory, bottom: mean trajectory tracking error, and right: mean hysteresis loop of transient phase. 


\section{C.2.2 LD Joint Data}
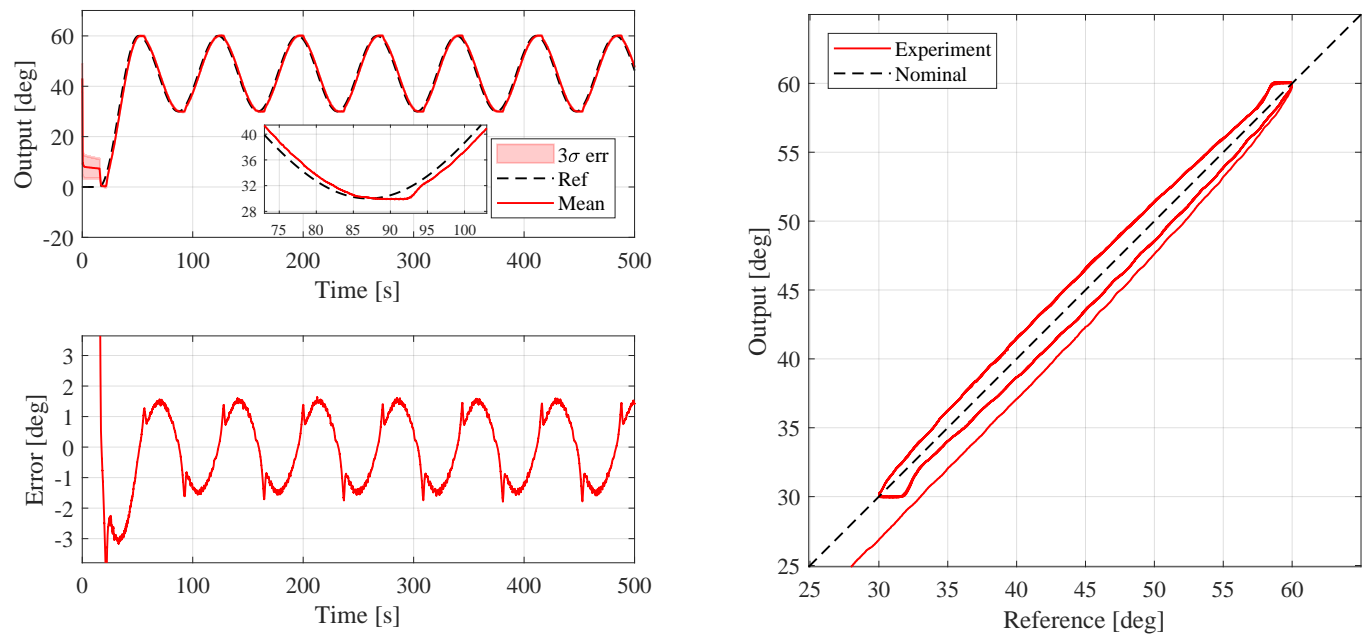

Figure C.16: LD joint performance for the Sine Wave manoeuvre in a straight arm configuration under PID control. Top: actual and desired proximal link angular trajectory, bottom: mean trajectory tracking error, and right: mean hysteresis loop of transient phase.
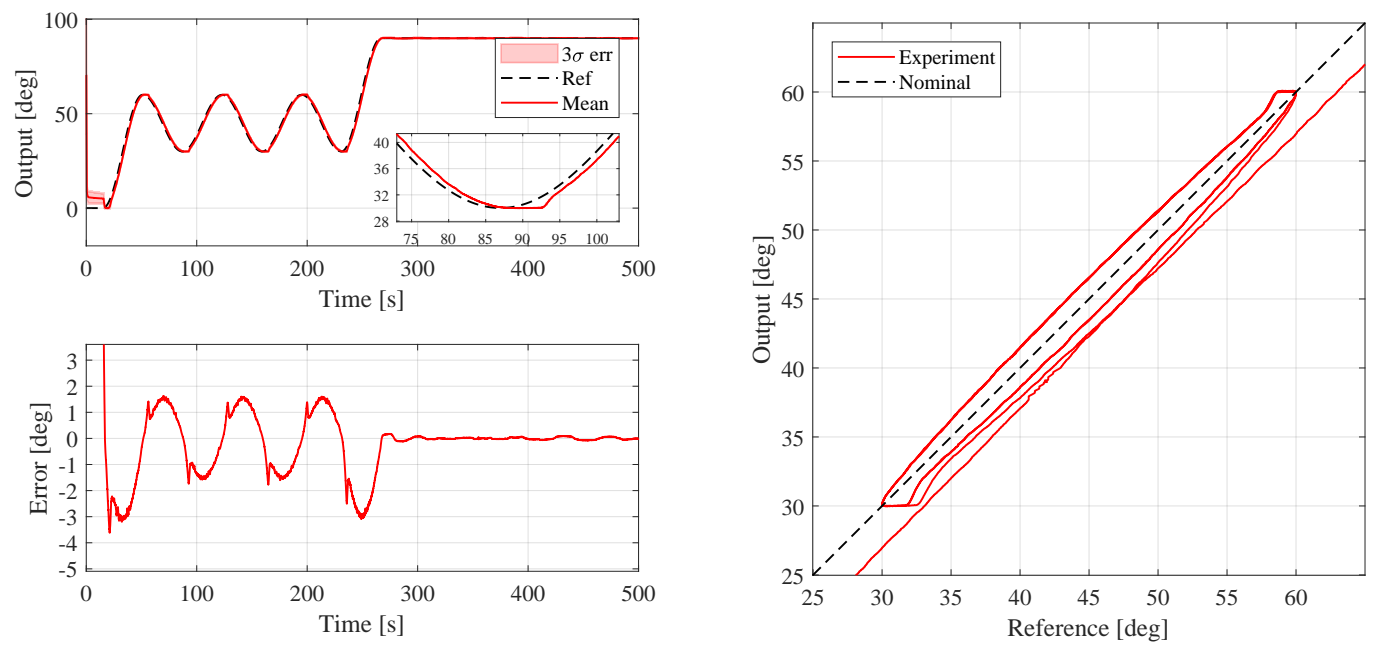

Figure C.17: LD joint performance for Manoeuvre 1 in a straight arm configuration under PID control. Top: actual and desired proximal link angular trajectory, bottom: mean trajectory tracking error, and right: mean hysteresis loop of transient phase. 

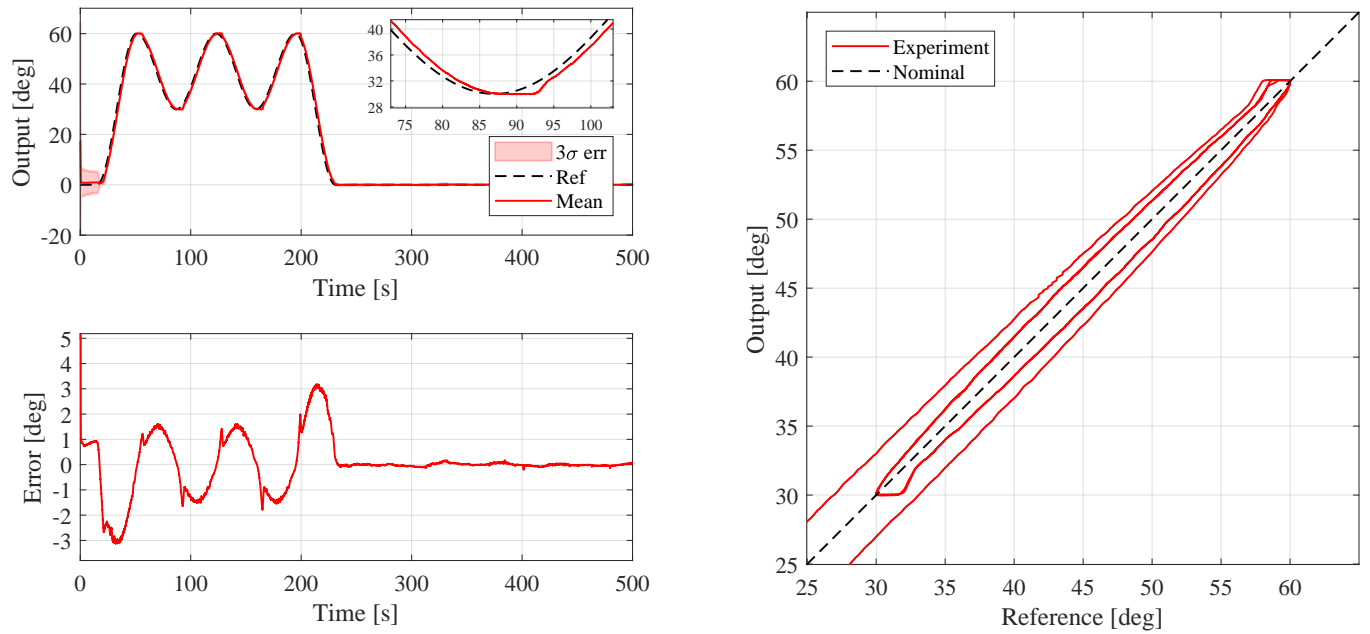

Figure C.18: LD joint performance for Manoeuvre 2 in a straight arm configuration under PID control. Top: actual and desired proximal link angular trajectory, bottom: mean trajectory tracking error, and right: mean hysteresis loop of transient phase.
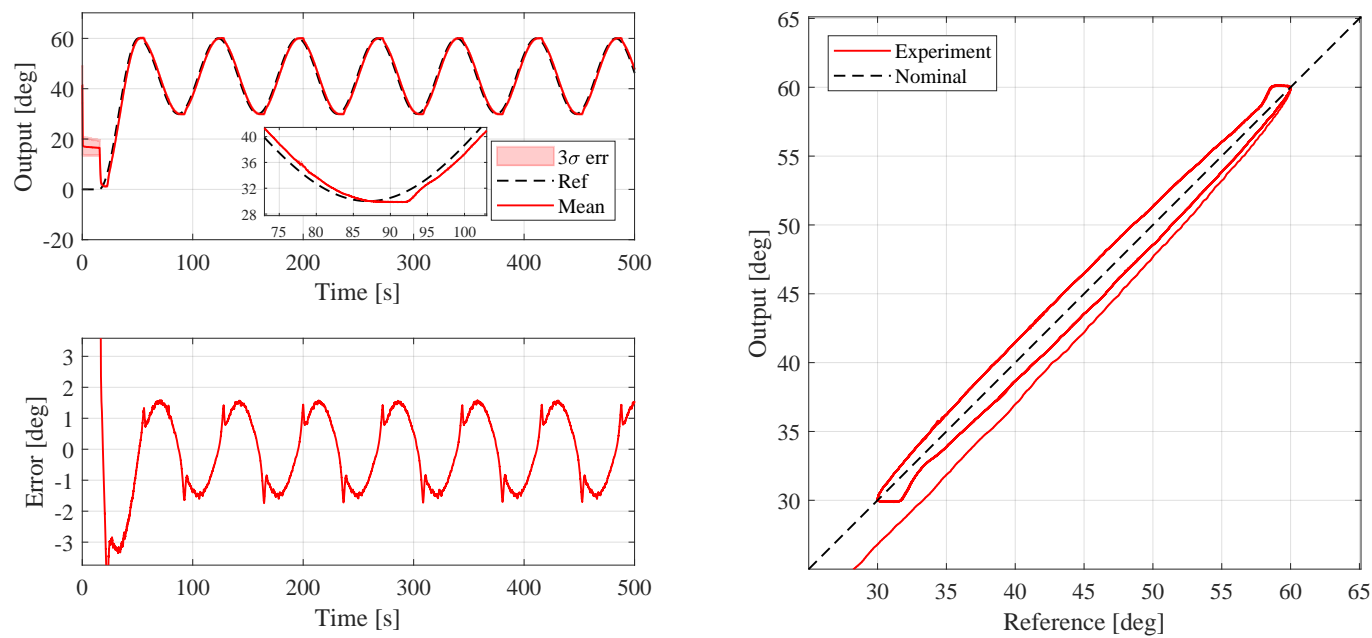

Figure C.19: LD joint performance for the Sine Wave manoeuvre in a bent arm configuration under PID control. Top: actual and desired proximal link angular trajectory, bottom: mean trajectory tracking error, and right: mean hysteresis loop of transient phase. 

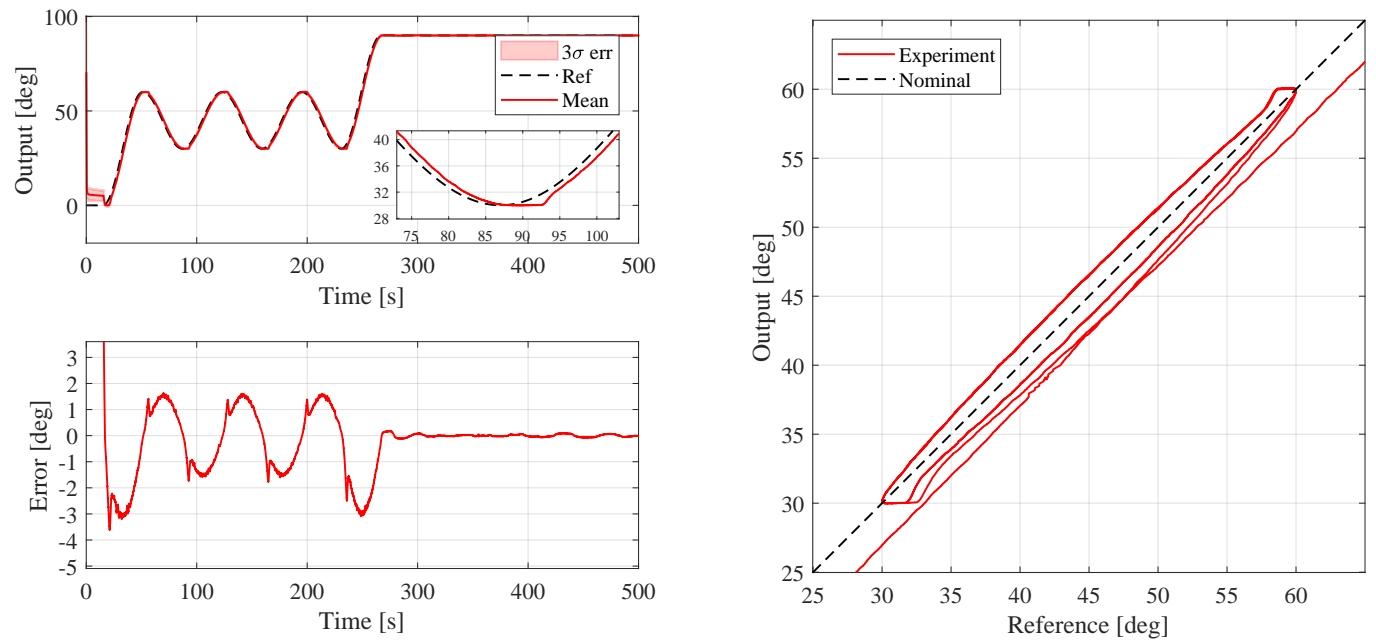

Figure C.20: LD joint performance for Manoeuvre 1 in a straight arm configuration under PID control. Top: actual and desired proximal link angular trajectory, bottom: mean trajectory tracking error, and right: mean hysteresis loop of transient phase.
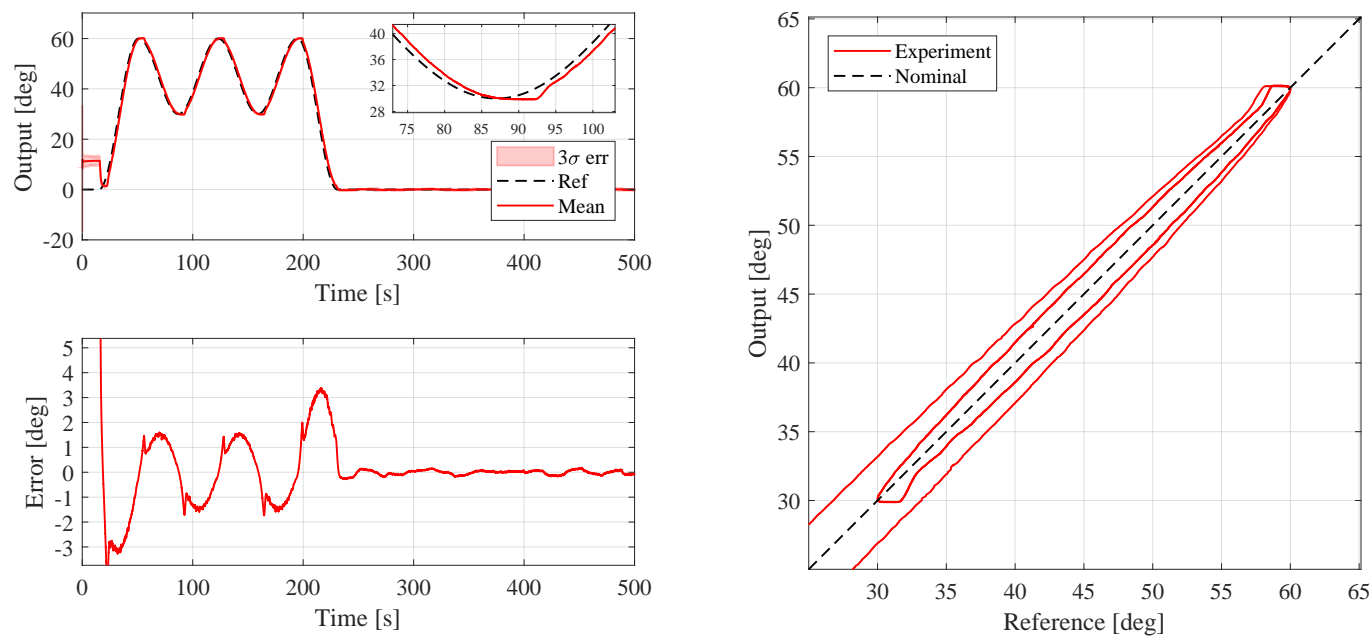

Figure C.21: LD joint performance for Manoeuvre 2 in a bent arm configuration under PID control. Top: actual and desired proximal link angular trajectory, bottom: mean trajectory tracking error, and right: mean hysteresis loop of transient phase. 

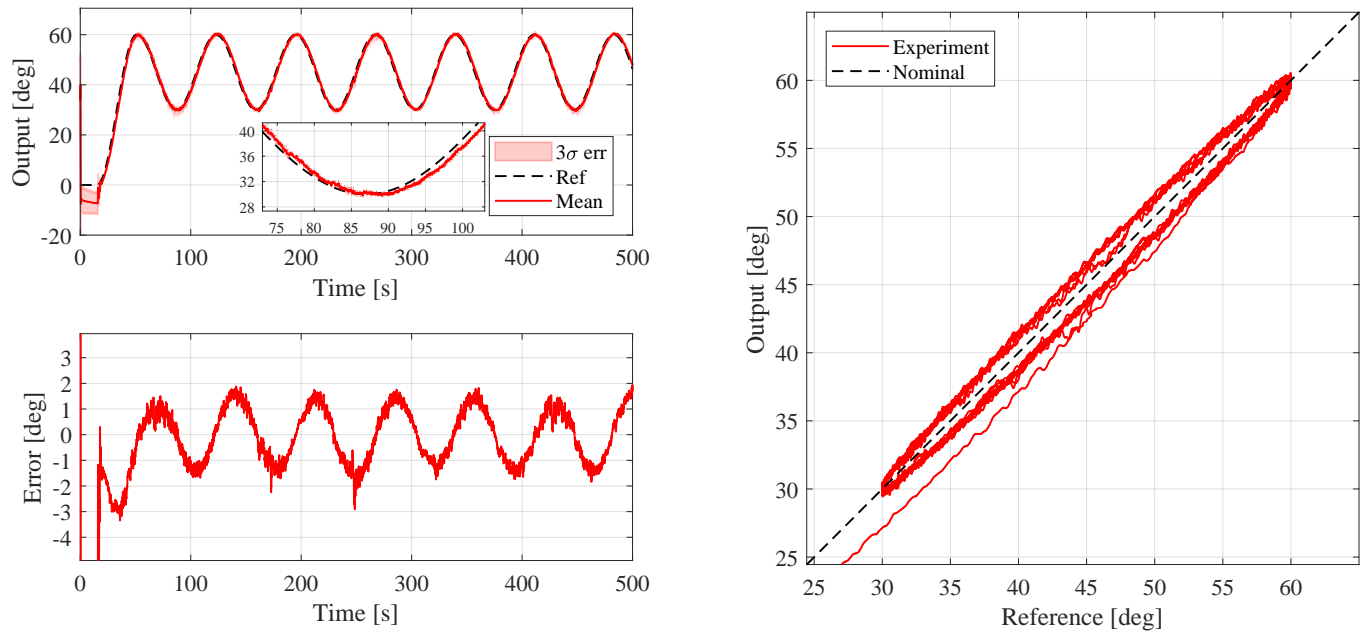

Figure C.22: LD joint performance with moving arm under PID control. Top: actual and desired proximal link angular trajectory, bottom: mean trajectory tracking error, and right: mean hysteresis loop of transient phase.

\section{C.3 AB Control}

\section{C.3.1 LP Joint Data}
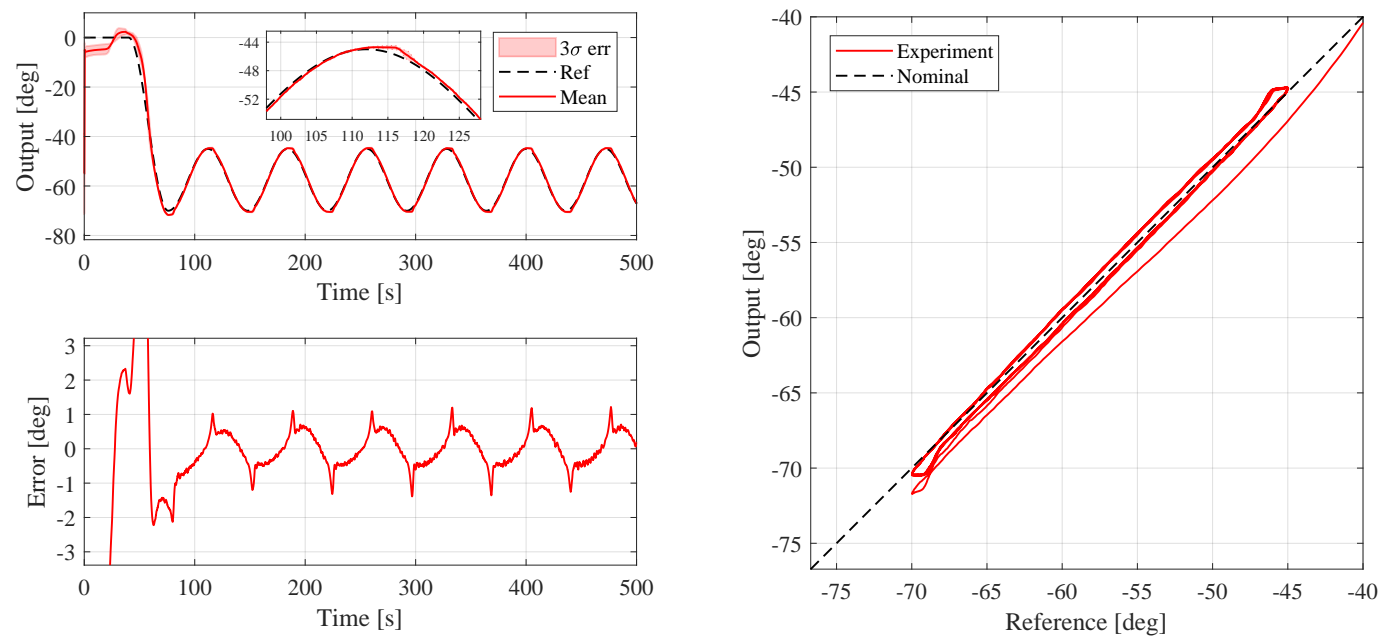

Figure C.23: LP joint performance for the Sine Wave manoeuvre in a straight arm configuration under AB control. Top: actual and desired proximal link angular trajectory, bottom: mean trajectory tracking error, and right: mean hysteresis loop of transient phase. 

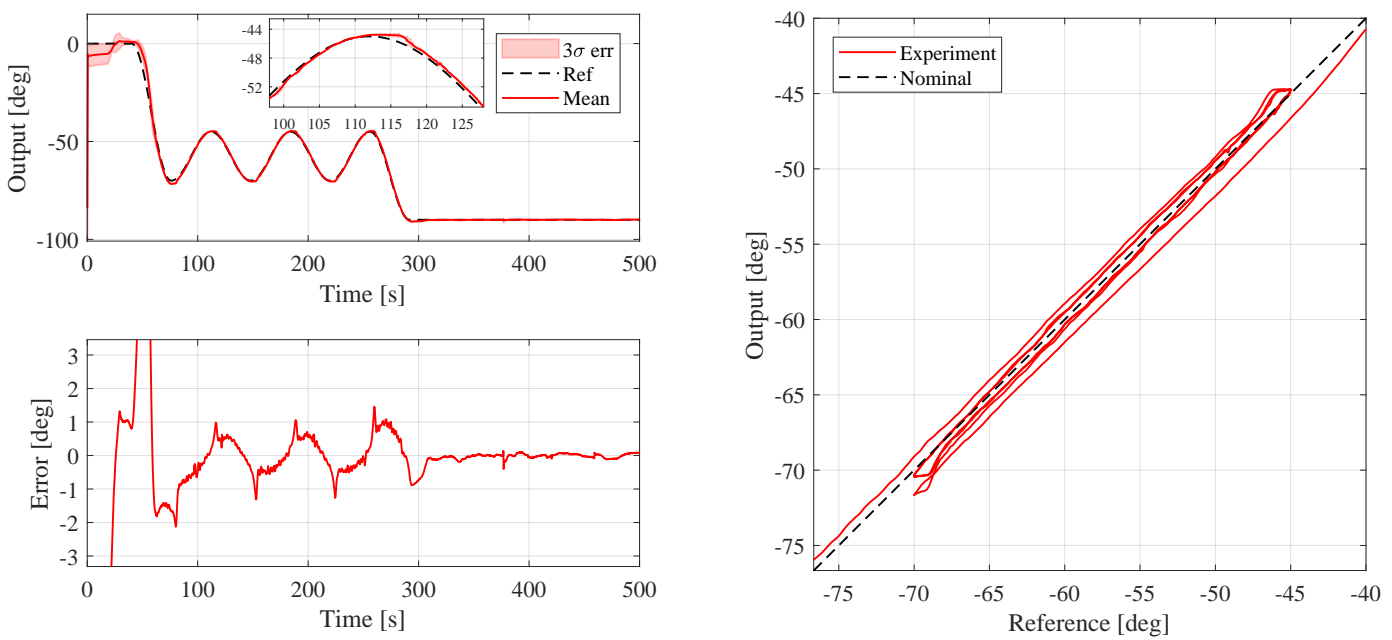

Figure C.24: LP joint performance for Manoeuvre 2 in a straight arm configuration under AB control. Top: actual and desired proximal link angular trajectory, bottom: mean trajectory tracking error, and right: mean hysteresis loop of transient phase.
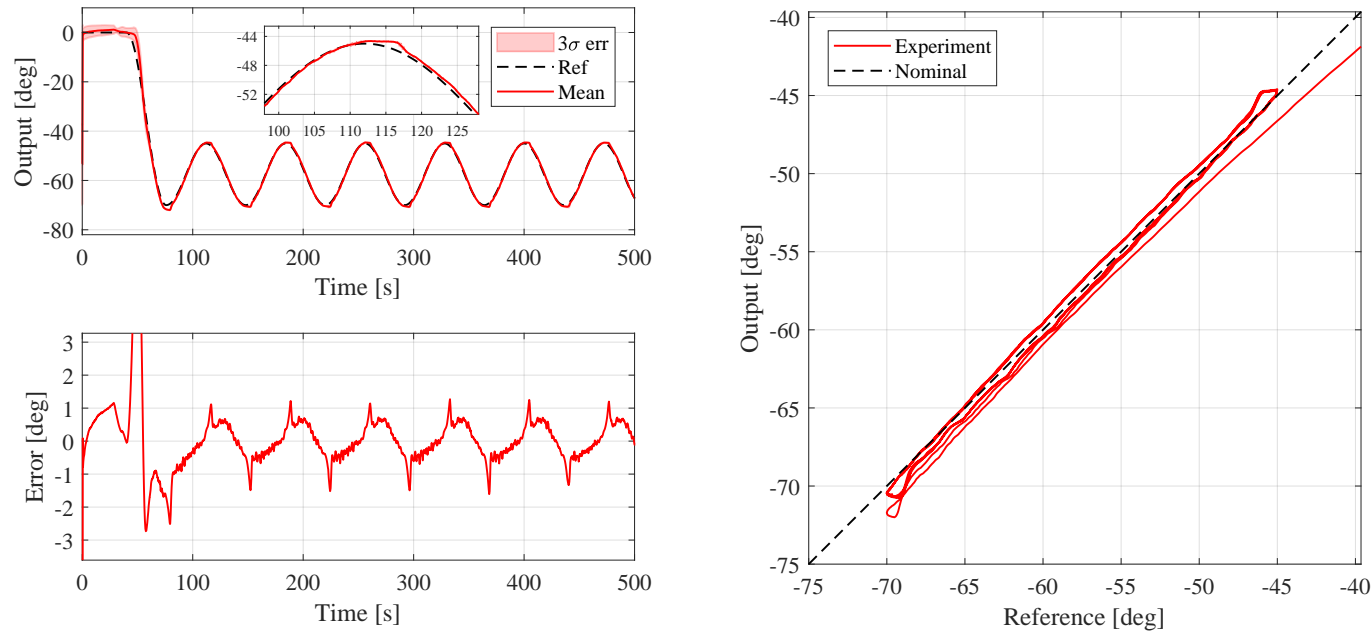

Figure C.25: LP joint performance for the Sine Wave manoeuvre in a bent arm configuration under AB control. Top: actual and desired proximal link angular trajectory, bottom: mean trajectory tracking error, and right: mean hysteresis loop of transient phase. 

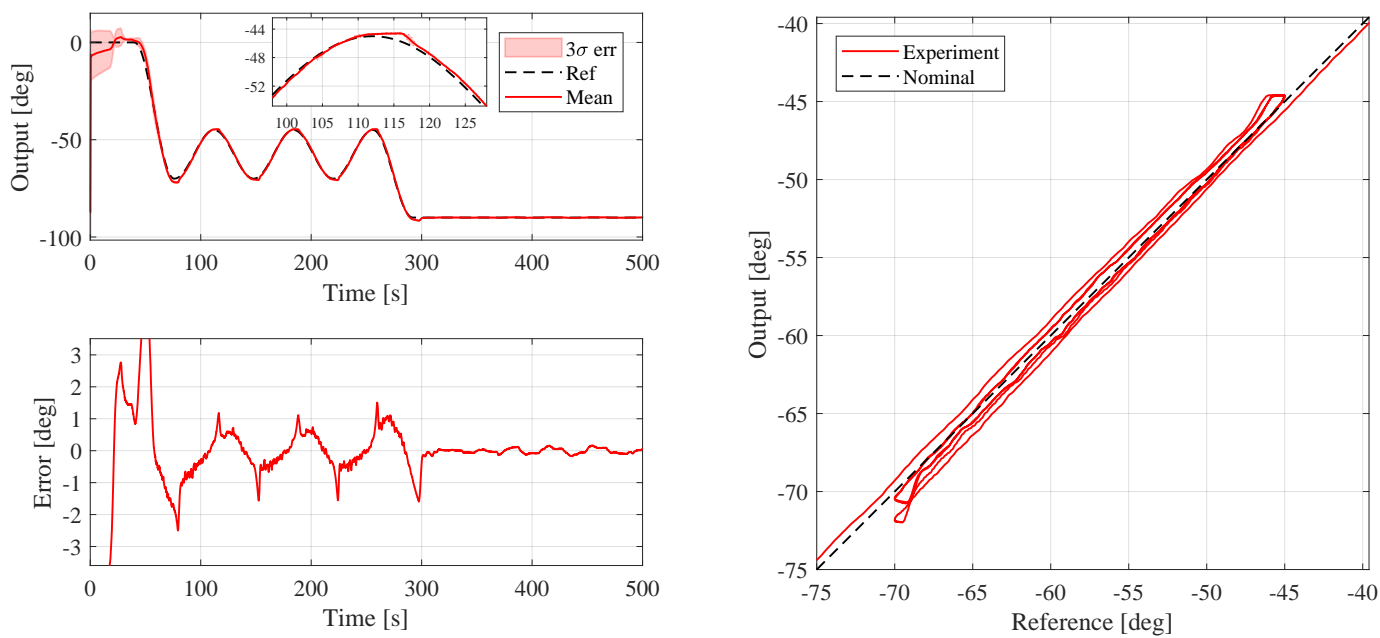

Figure C.26: LP joint performance for Manoeuvre 2 in a bent arm configuration under AB control. Top: actual and desired proximal link angular trajectory, bottom: mean trajectory tracking error, and right: mean hysteresis loop of transient phase.

\section{C.3.2 LD Joint Data}
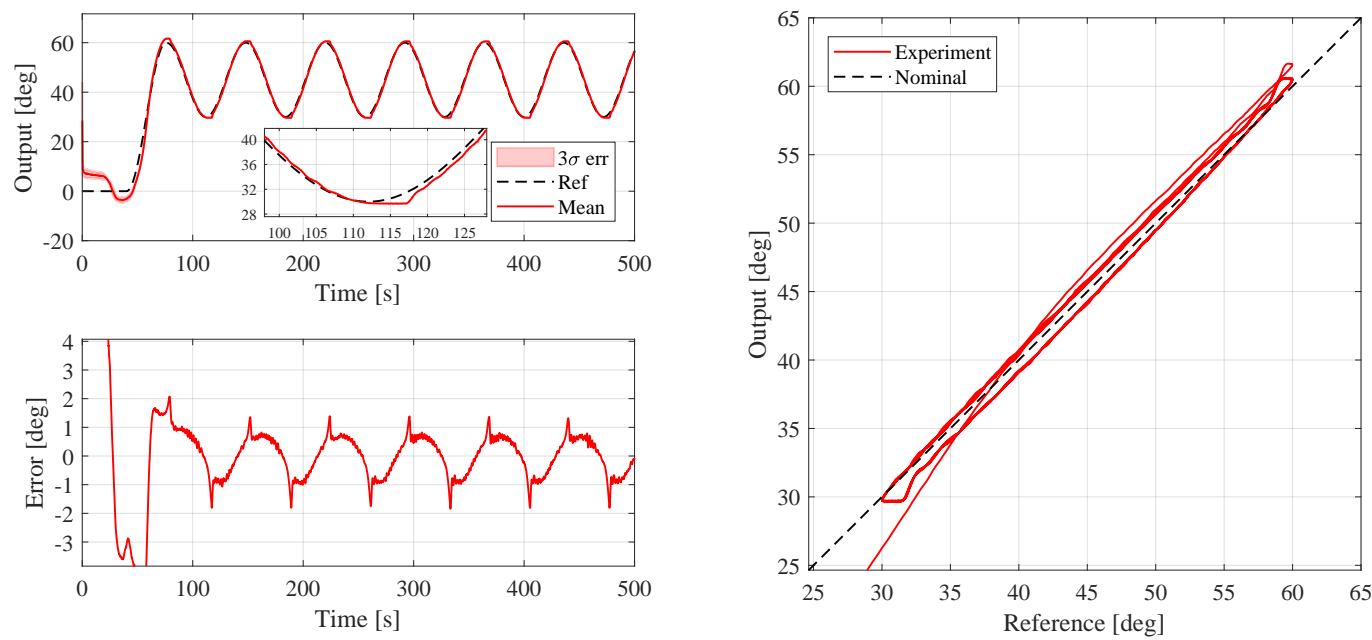

Figure C.27: LD joint performance for the Sine Wave manoeuvre in a straight arm configuration under AB control. Top: actual and desired proximal link angular trajectory, bottom: mean trajectory tracking error, and right: mean hysteresis loop of transient phase. 

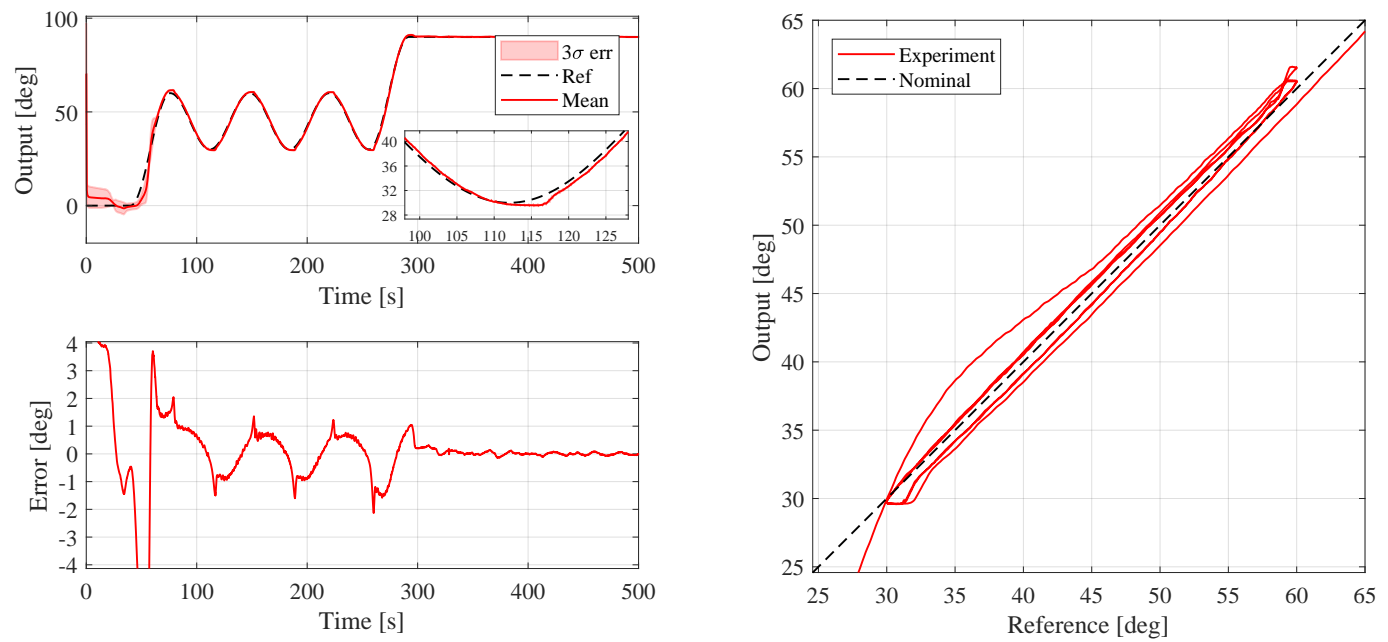

Figure C.28: LD joint performance for Manoeuvre 1 in a straight arm configuration under AB control. Top: actual and desired proximal link angular trajectory, bottom: mean trajectory tracking error, and right: mean hysteresis loop of transient phase.
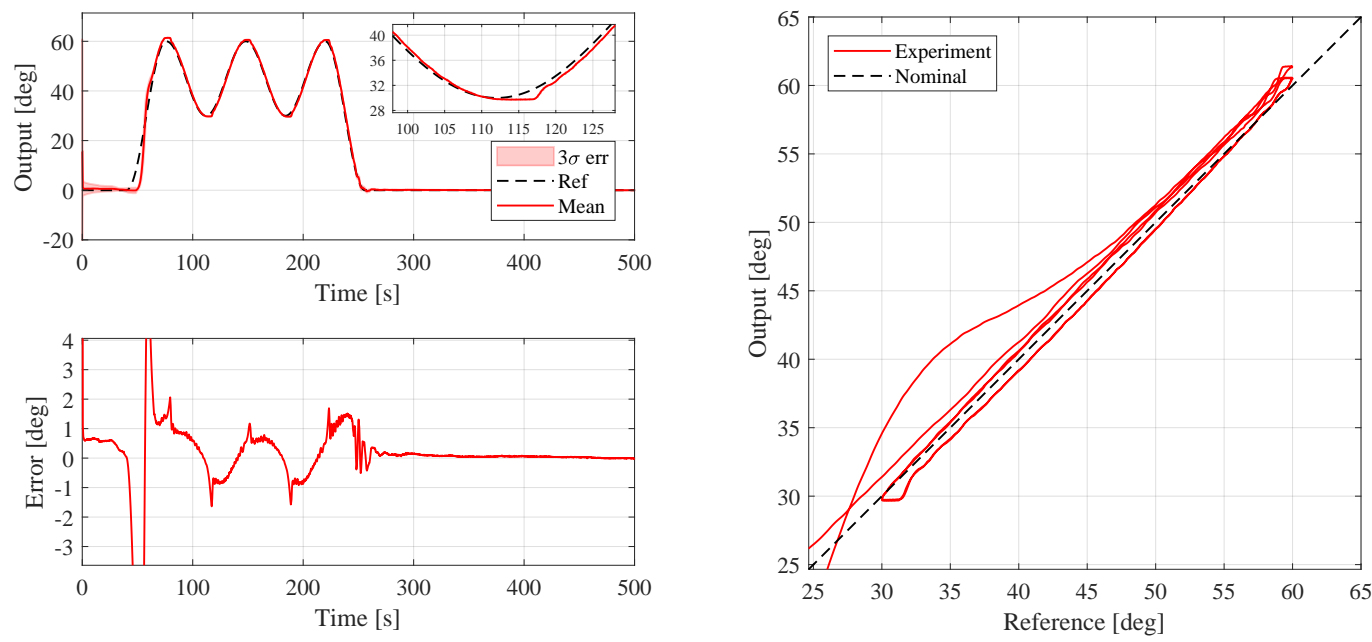

Figure C.29: LD joint performance for Manoeuvre 2 in a straight arm configuration under AB control. Top: actual and desired proximal link angular trajectory, bottom: mean trajectory tracking error, and right: mean hysteresis loop of transient phase. 

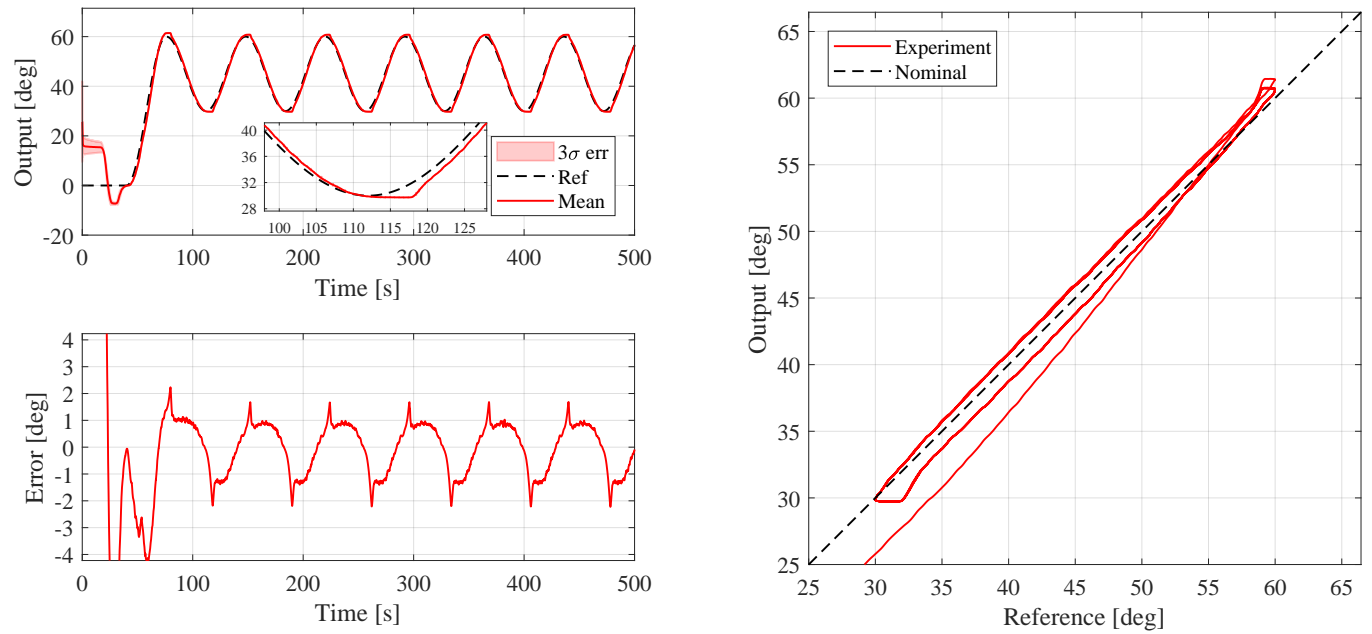

Figure C.30: LD joint performance for the Sine Wave manoeuvre in a bent arm configuration under AB control. Top: actual and desired proximal link angular trajectory, bottom: mean trajectory tracking error, and right: mean hysteresis loop of transient phase.
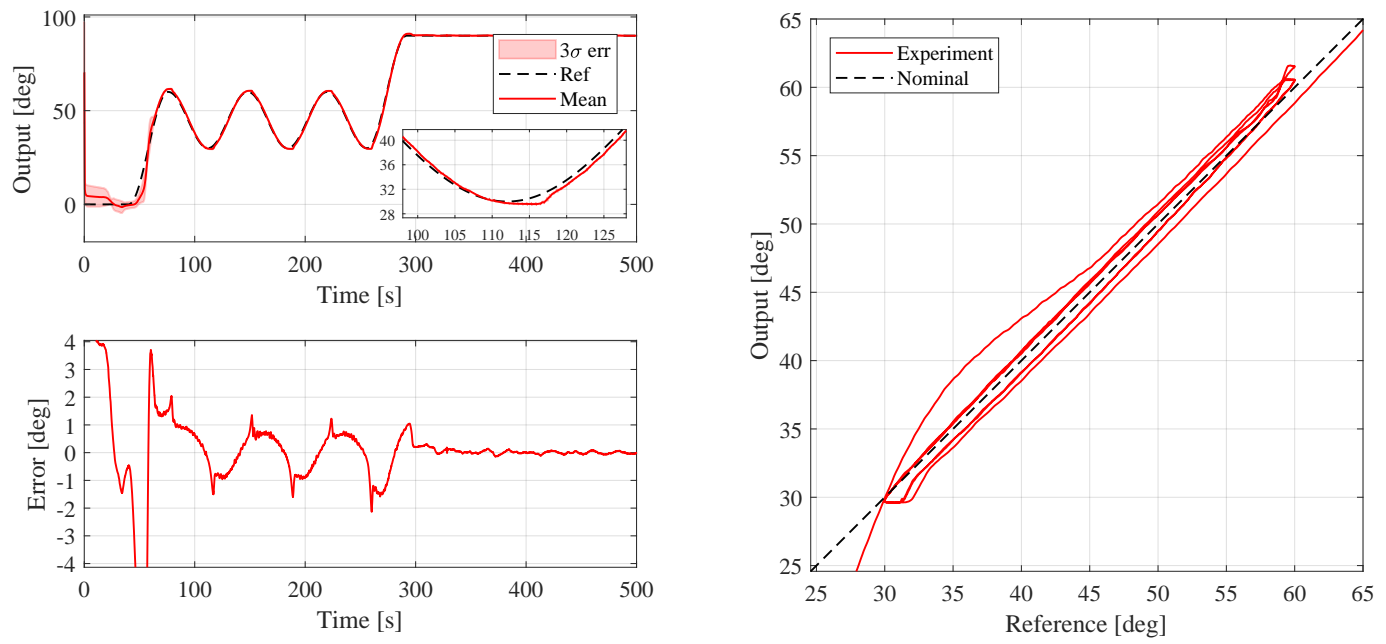

Figure C.31: LD joint performance for Manoeuvre 1 in a straight arm configuration under AB control. Top: actual and desired proximal link angular trajectory, bottom: mean trajectory tracking error, and right: mean hysteresis loop of transient phase. 

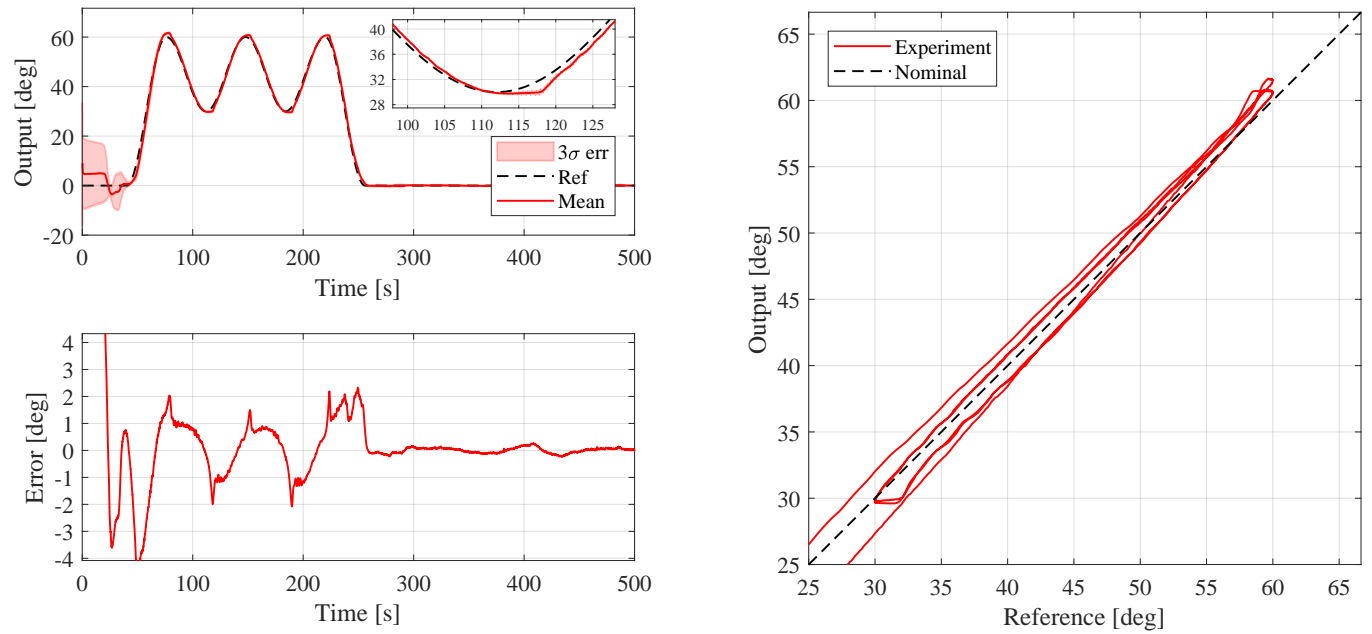

Figure C.32: LD joint performance for Manoeuvre 2 in a bent arm configuration under AB control. Top: actual and desired proximal link angular trajectory, bottom: mean trajectory tracking error, and right: mean hysteresis loop of transient phase.
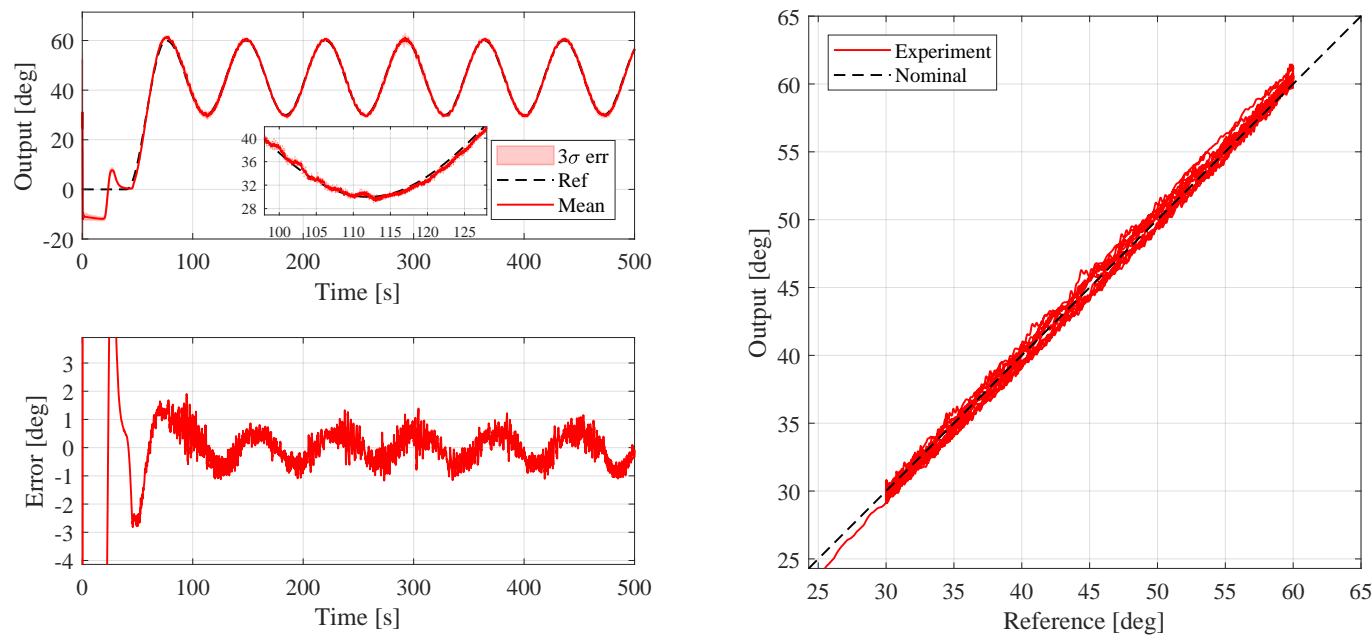

Figure C.33: LD joint performance with moving arm under AB control. Top: actual and desired proximal link angular trajectory, bottom: mean trajectory tracking error, and right: mean hysteresis loop of transient phase. 


\section{C.4 $\mathcal{L}_{1}$ Control}

\section{C.4.1 LP Joint Data}
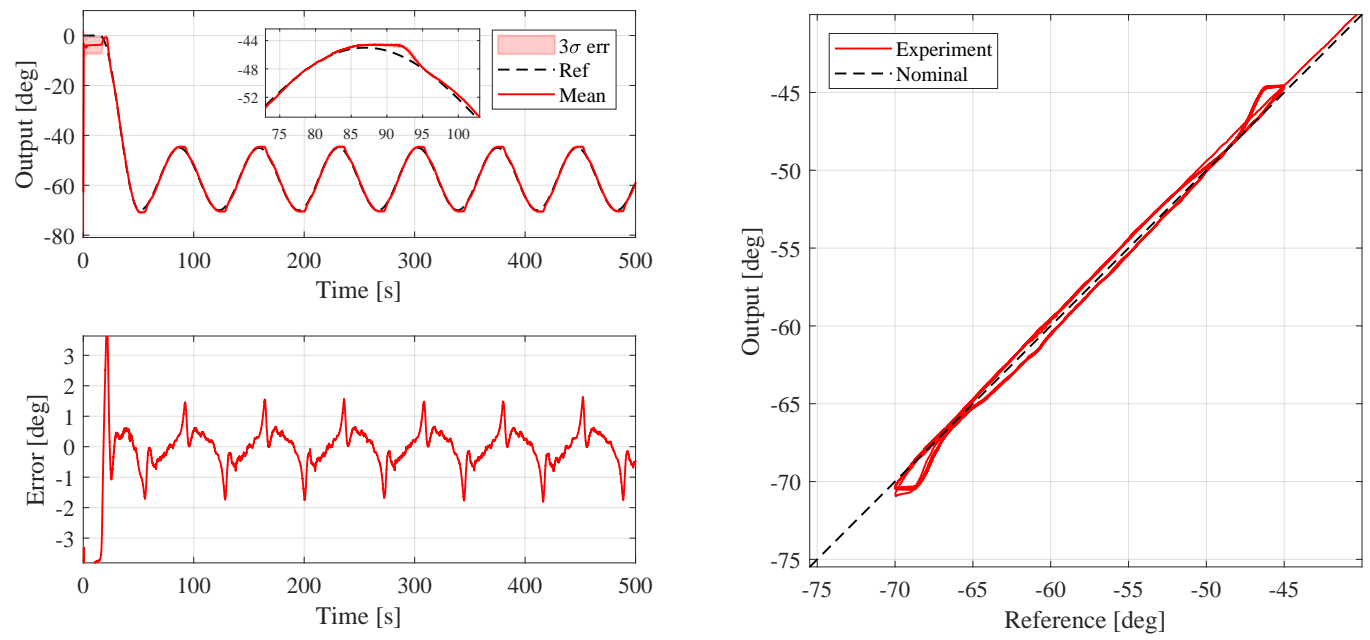

Figure C.34: LP joint performance for the Sine Wave manoeuvre in a straight arm configuration under $\mathcal{L}_{1}$ control. Top: actual and desired proximal link angular trajectory, bottom: mean trajectory tracking error, and right: mean hysteresis loop of transient phase.
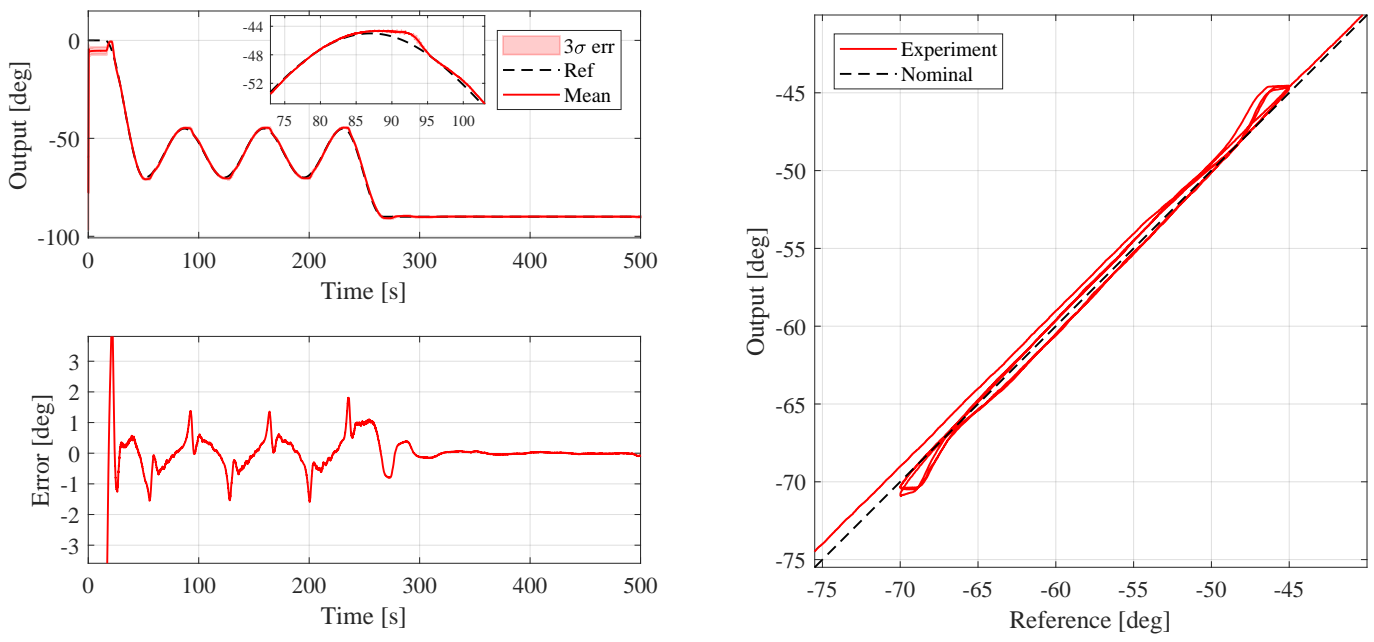

Figure C.35: LP joint performance for Manoeuvre 2 in a straight arm configuration under $\mathcal{L}_{1}$ control. Top: actual and desired proximal link angular trajectory, bottom: mean trajectory tracking error, and right: mean hysteresis loop of transient phase. 

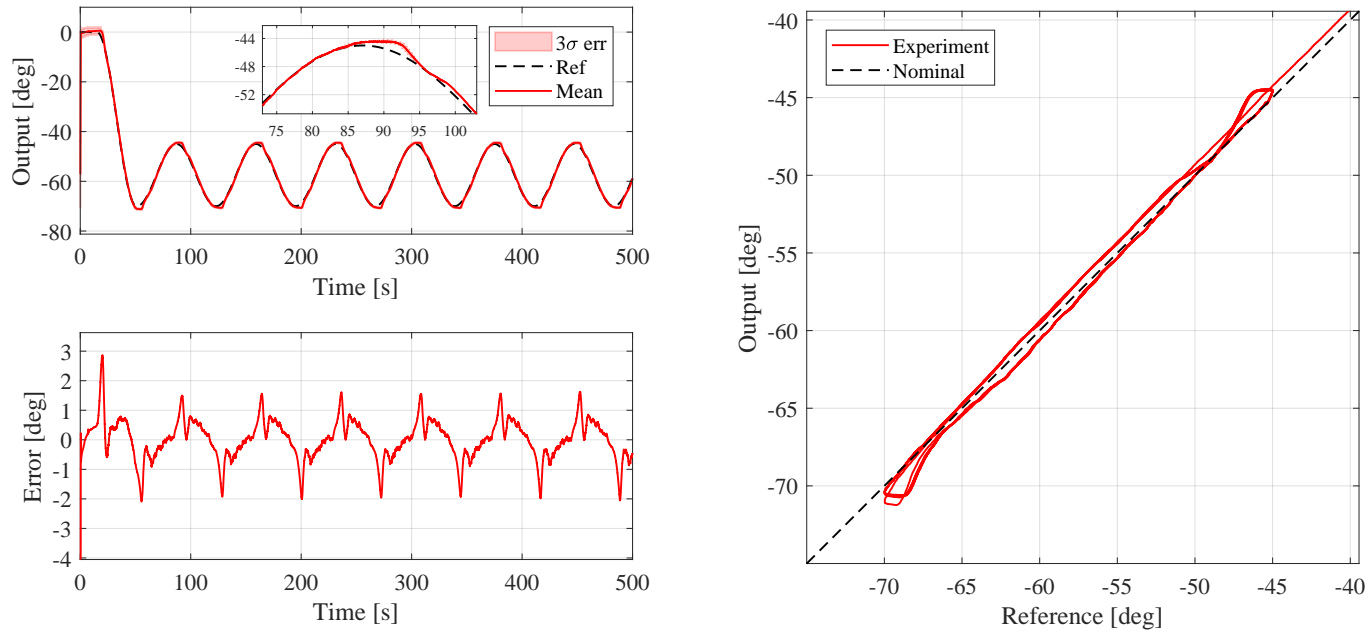

Figure C.36: LP joint performance for the Sine Wave manoeuvre in a bent arm configuration under $\mathcal{L}_{1}$ control. Top: actual and desired proximal link angular trajectory, bottom: mean trajectory tracking error, and right: mean hysteresis loop of transient phase.
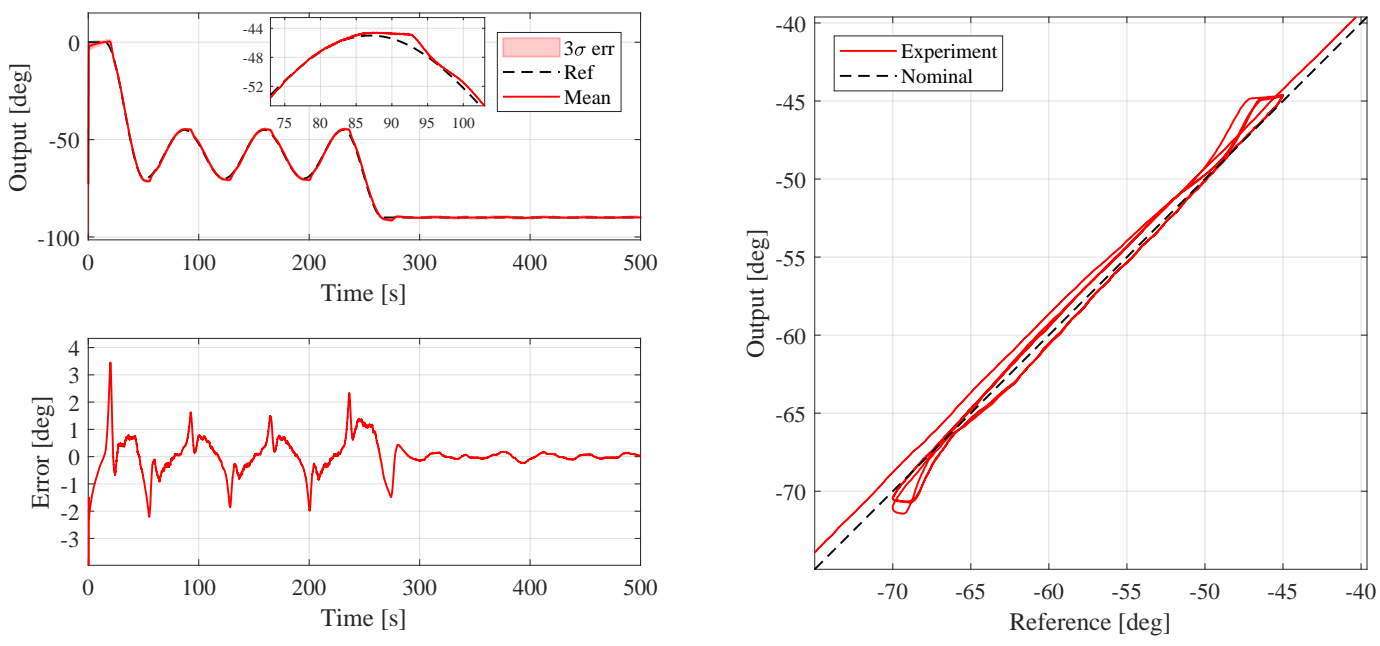

Figure C.37: LP joint performance for Manoeuvre 2 in a bent arm configuration under $\mathcal{L}_{1}$ control. Top: actual and desired proximal link angular trajectory, bottom: mean trajectory tracking error, and right: mean hysteresis loop of transient phase. 


\section{C.4.2 LD Joint Data}
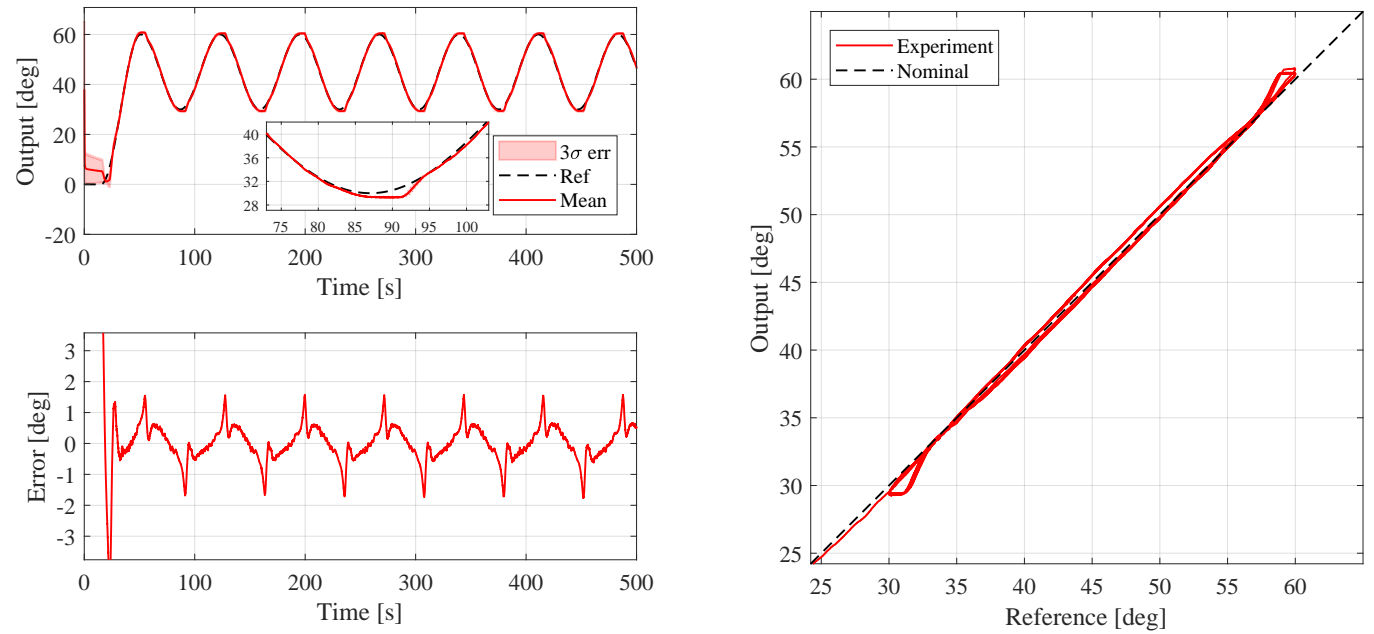

Figure C.38: LD joint performance for the Sine Wave manoeuvre in a straight arm configuration under $\mathcal{L}_{1}$ control. Top: actual and desired proximal link angular trajectory, bottom: mean trajectory tracking error, and right: mean hysteresis loop of transient phase.
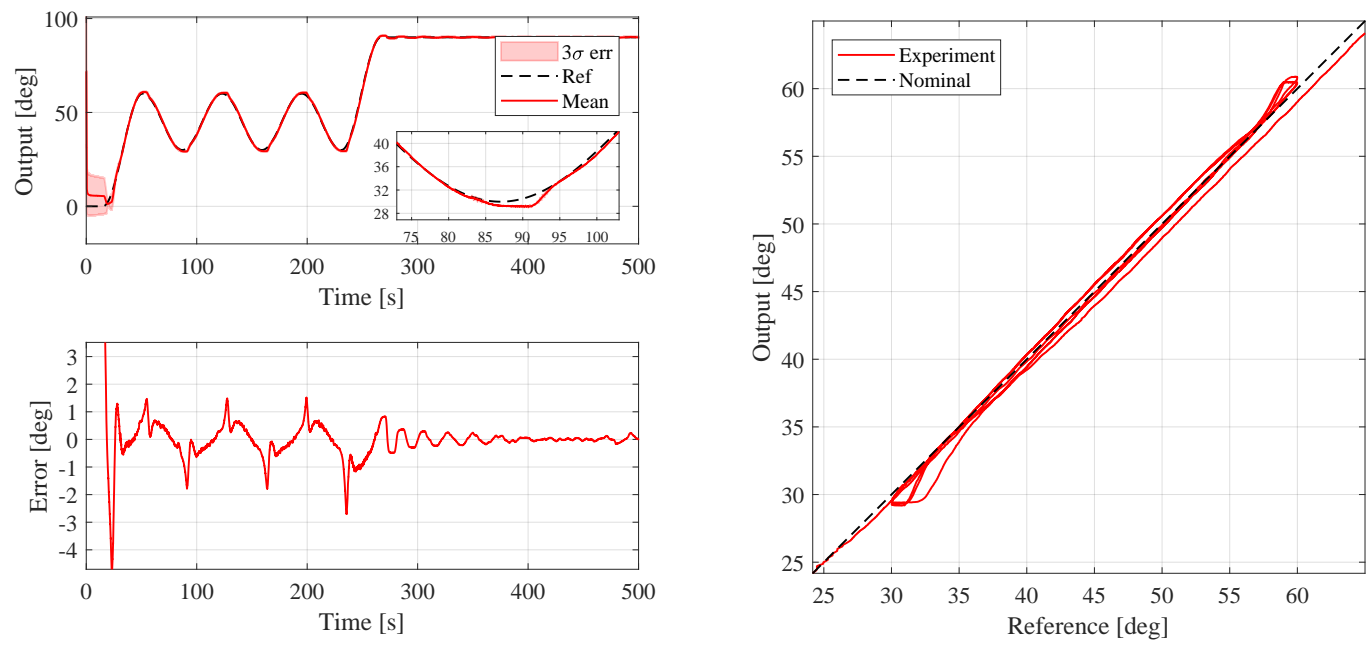

Figure C.39: LD joint performance for Manoeuvre 1 in a straight arm configuration under $\mathcal{L}_{1}$ control. Top: actual and desired proximal link angular trajectory, bottom: mean trajectory tracking error, and right: mean hysteresis loop of transient phase. 

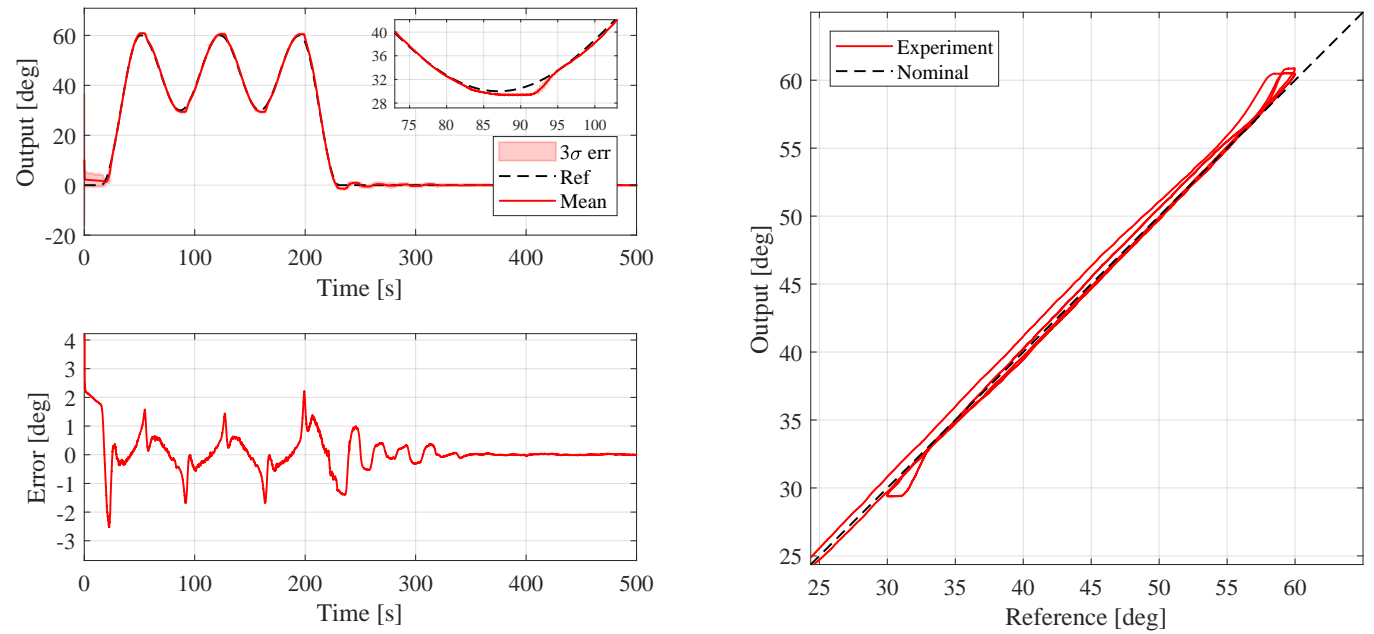

Figure C.40: LD joint performance for Manoeuvre 2 in a straight arm configuration under $\mathcal{L}_{1}$ control. Top: actual and desired proximal link angular trajectory, bottom: mean trajectory tracking error, and right: mean hysteresis loop of transient phase.
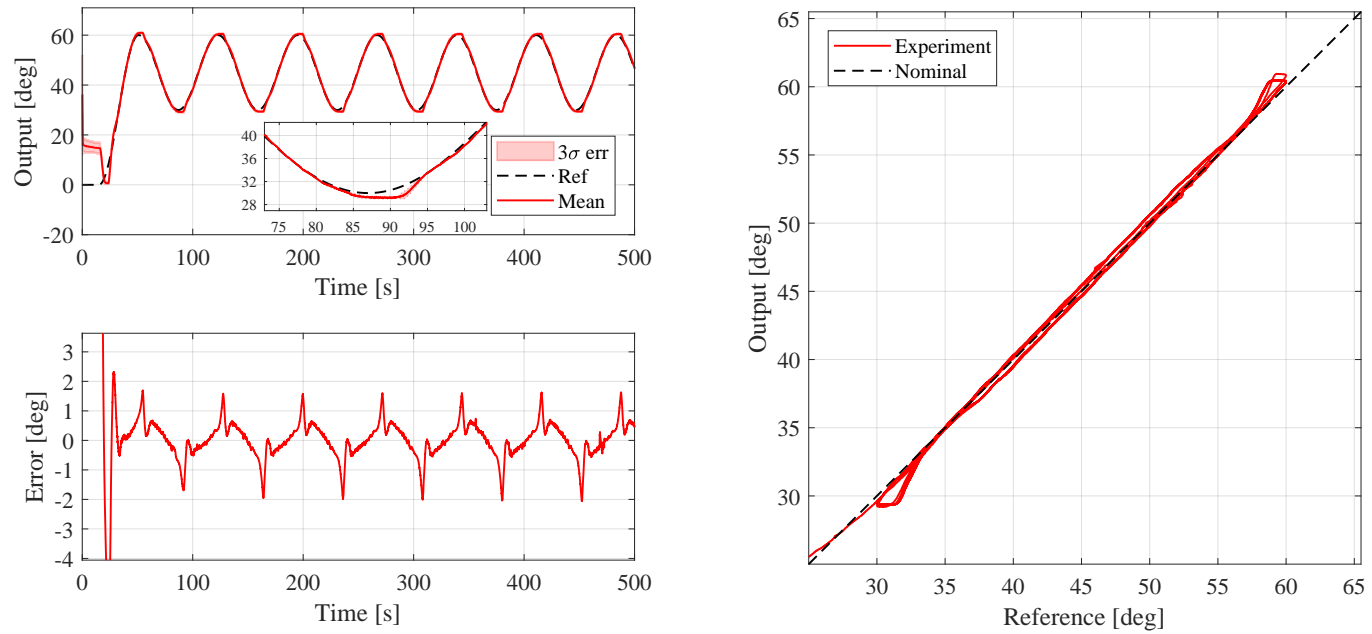

Figure C.41: LD joint performance for the Sine Wave manoeuvre in a bent arm configuration under $\mathcal{L}_{1}$ control. Top: actual and desired proximal link angular trajectory, bottom: mean trajectory tracking error, and right: mean hysteresis loop of transient phase. 

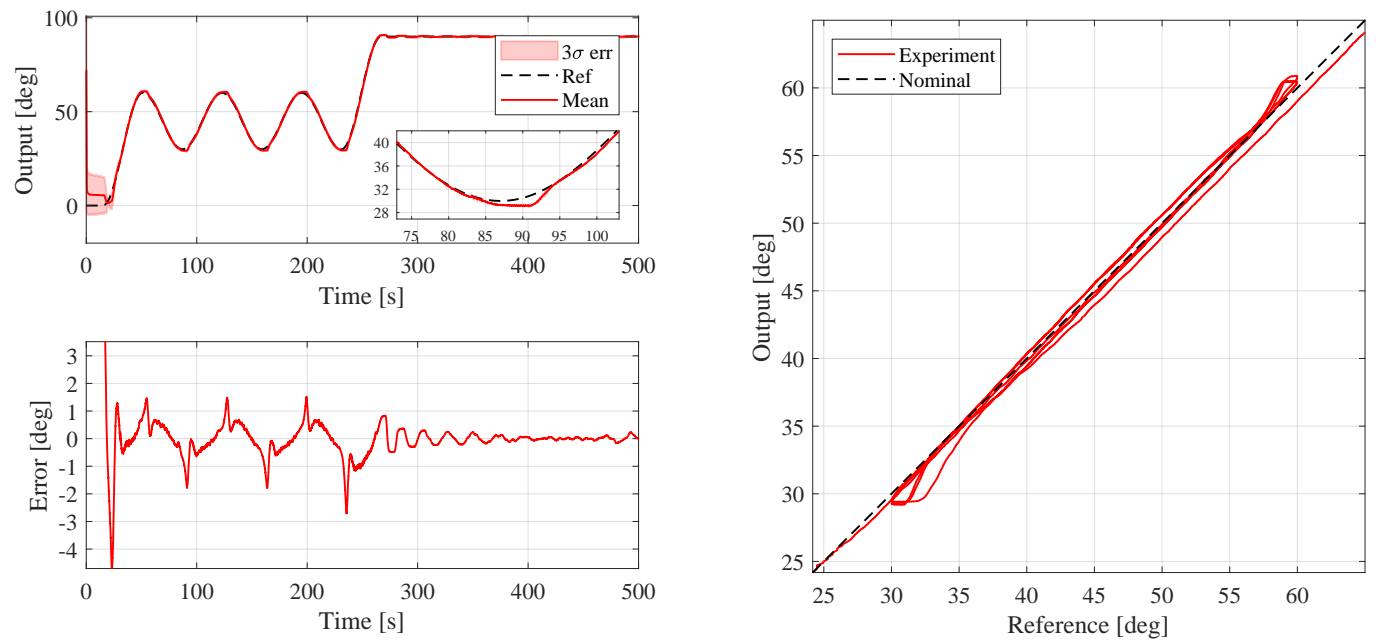

Figure C.42: LD joint performance for Manoeuvre 1 in a straight arm configuration under $\mathcal{L}_{1}$ control. Top: actual and desired proximal link angular trajectory, bottom: mean trajectory tracking error, and right: mean hysteresis loop of transient phase.
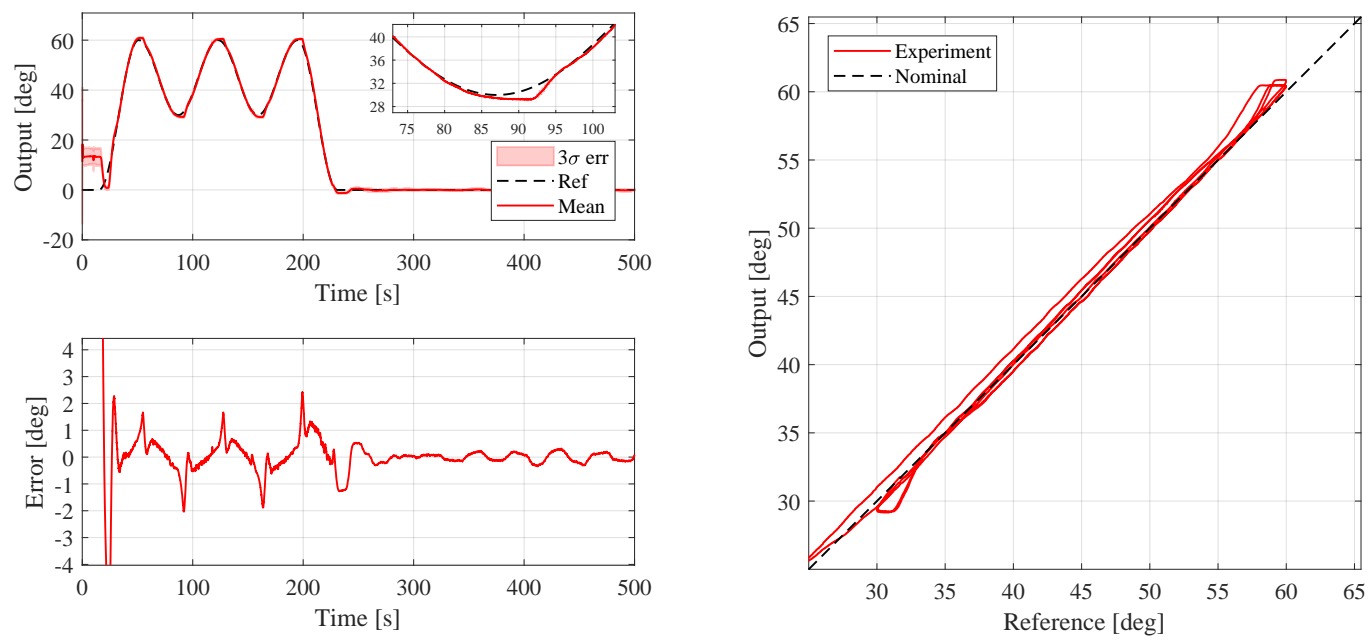

Figure C.43: LD joint performance for Manoeuvre 2 in a bent arm configuration under $\mathcal{L}_{1}$ control. Top: actual and desired proximal link angular trajectory, bottom: mean trajectory tracking error, and right: mean hysteresis loop of transient phase. 

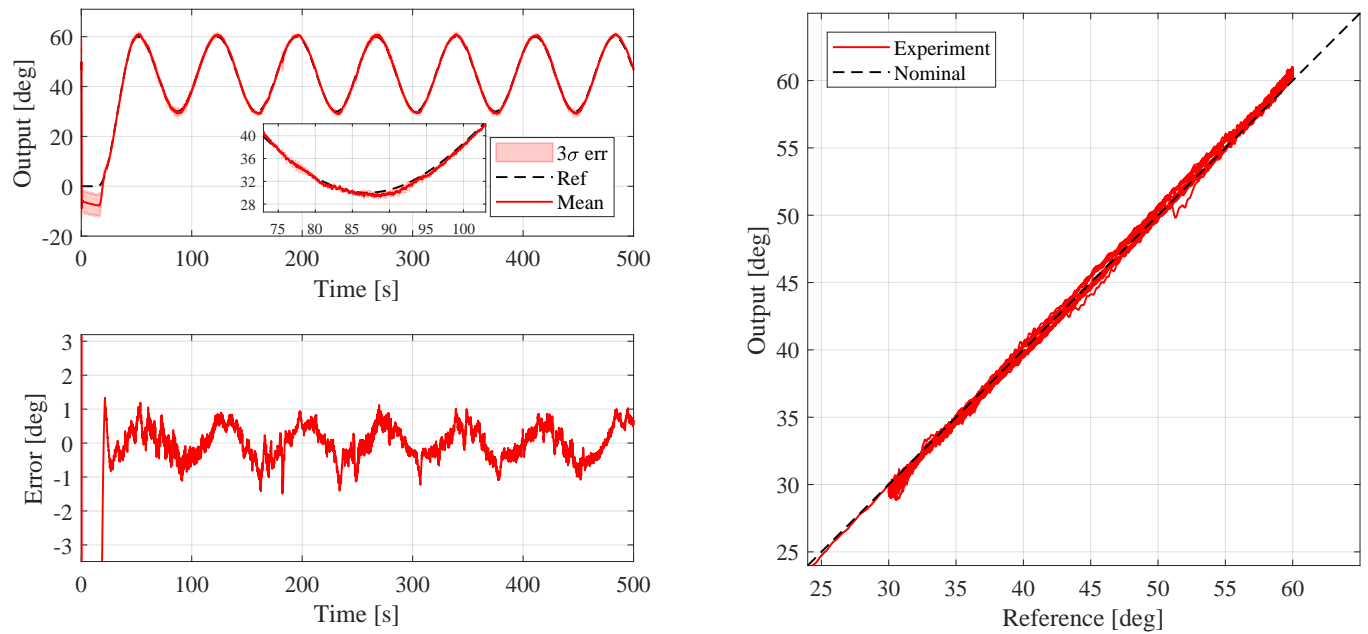

Figure C.44: LD joint performance with moving arm under $\mathcal{L}_{1}$ control. Top: actual and desired proximal link angular trajectory, bottom: mean trajectory tracking error, and right: mean hysteresis loop of transient phase. 